This document is downloaded from DR-NTU (https://dr.ntu.edu.sg) Nanyang Technological University, Singapore.

Optimal sensor placement for model-based fault detectability and isolability

Chi, Guoyi

2014

https://hdl.handle.net/10356/60470

https://doi.org/10.32657/10356/60470 


\section{Optimal Sensor Placement for Model-Based Fault Detectability and Isolability}
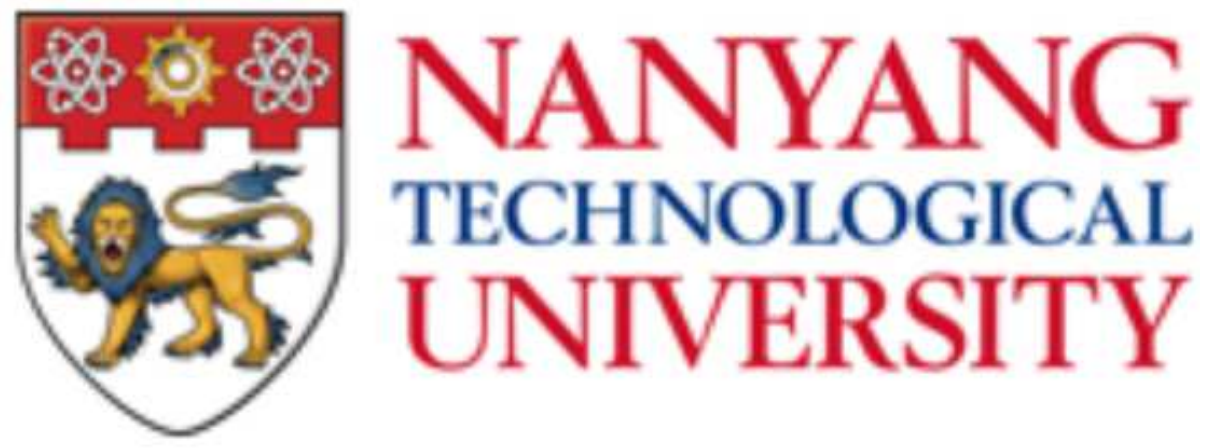

Chi Guoyi

School of Electrical \& Electronic Engineering

A thesis submitted to the Nanyang Technological University in fulfillment of the requirements for the degree of

Doctor of Philosophy

May 2014 



\section{$\underline{\text { Statement of Originality }}$}

I hereby certify that the work embodied in this thesis is the result of original research and has not been submitted for a higher degree to any other University or Institution. 
To my dear parents,

for their encouragement and love. 


\section{Acknowledgement}

In the completion of my research and this dissertation, I would like to express my sincere gratitude to Professor Wang Danwei, my supervisor, for his constant encouragement, support and guidance throughout the study. His help makes research like this possible. His discussions on every issue were always helpful and put forward the way to the solutions. I also like to express my gratitude to my co-supervisor Dr. Le Tung from Singapore Institute of Manufacturing Technology (SIMTech).

Of course, I am grateful to my parents for their patience and love. Without them this work would never have come into existence.

Also, I would like to thank all members in our research group of Health Monitoring, Fault Diagnosis and Prognosis for Complex Systems, Dr. Yu Ming, Mr. Zhu Senqiang, Ms. Majan Alavi as well as Dr. Luo Ming from SIMTech for all your discussions and suggestions. My gratitude also goes to the technical staffs at Intelligent Robotics Laboratory for their help and support.

Finally, I wish to thank the following: Chen Tao (my last supervisor); Wang Ke, Yan Wenjin (my mates in the last research topic); Li Xiang, Fan Haijin, Liu Tianqi, Shen Qiang, etc. (all PhDs in my laboratory); all my roommates. They made my doctoral studies at Singapore very pleasant. 



\section{Abstract}

This dissertation investigates the sensor placement issue in a dynamic system for fault detectability and isolability. A degree of detectability (respectively, isolability) which accounts for faults in a dynamic system under consideration, is defined and then optimized over the admissible set of candidate sensors. In this study, emphasis will be placed upon how to achieve fault detectability and isolability in a first principle model using the least number of candidate sensors.

The definitions for model-based fault detectability and isolability have two types, and they are made according to whether or not have reference to residual generators. One is termed performance-based fault detectability and isolability which is expressed in terms of the signature of the faults on the residuals. Alternatively, the other is named intrinsic fault detectability and isolability that is known as a structural property of the investigated system. The latter type (of fault detectability and isolability) captures the signature of the faults on the system other than the residuals. This dissertation studies the optimization problem of sensor placement with respect to both types: performance-based and intrinsic.

One research work presented in this dissertation is to propose a novel and highly efficient approach of sensor placement for performance-based fault detectability and fault isolability. In the parity space, all possible residuals, called analytical redundancy relations (ARRs), are generated by some sophisticated methods. This new approach rests on the basic facts that faults and candidate sensors are embedded in 
ARRs. Therefore, fault detectability is expressed in terms of a set of ARRs which response to a specified fault; meanwhile fault isolability is to find out a unique set of ARRs regarding to a specified fault. From this discovery, the sensor placement problem constraint (fault detectability and isolability) can be expressed as a minimal isolating (MI) set where each fault under consideration is isolable. Immediately, the senor placement problem is formulated as an optimization problem with respect to MI sets. For high-efficiency, a low complexity dynamic programming (LCDP) is developed and it serves to seek the optimal MI set for system faults (which is related to the least number of candidate sensors). Besides isolability of system faults, sensor faults are taken into consideration. Note that sensor faults are always varied as different sensor configurations are assigned to system faults. Then, a dedicated procedure is designed to solve this issue. This approach can solve the sensor placement issue for isolability of system faults and sensor faults in a linear or nonlinear model. However, the approach involves the risk that a lot of ARRs need to be constructed and most of them are impossibly utilized in the design of a fault diagnosis system.

To completely avoid generating residuals, sensor placement for intrinsic fault detectability and isolability is exploited. Until now, most of the publications discuss intrinsic fault detectability and isolability in a linear dynamic system. In this dissertation, the bond graph modelling technique is used to model a linear dynamic system. Different from the state space representation, graphical connections between faults and junctions are built by causal paths on a bond graph. Causal paths provide a powerful tool to analyze which junction can be used for fault detectability and isolability. So the sensor placement issue is restricted by junctions. Incorporating the restriction of junctions into fault detectability and isolability is investigated and thus relevant conditions of fault detectability and isolability are provided. Using fault detectability and isolability conditions, a sensor placement algorithm is developed to determine an optimal set of junctions that should be equipped with sensors. In 
this sensor placement algorithm, the basic building block is realized by solving a set of differential-algebraic equations (DAEs) which results from constitutive relations of a bond graph. For instance, if $Q$ faults are considered for isolability, this basic building block need to be invoked $\frac{Q^{2}+Q}{2}$ times. Consequently, the sensor placement algorithm is computationally intensive.

To design a more efficient algorithm for sensor placement, the structural characteristics of a bond graph are further investigated. With the aid of causal paths, this study provides a simpler condition for intrinsic fault detectability which is a necessary and sufficient condition. Based on this simpler condition for fault detectability, this study derives another simpler condition for distinguishability of two faults. However, this fault distinguishability condition is a necessary condition so as not to directly determine where should place sensors. Therefore, this fault distinguishability condition serves as the basis of the optimization formulation for isolability on a fault set. For high-efficiency, a dynamic programming algorithm is developed to attain the optimal solution from a pre-enumerated set of junctions. 



\section{Contents}

Acknowledgements $\quad$ i

Abstract

List of Contents vii

List of Figures $\quad$ xi

List of Tables $\quad$ xiii

List of Algorithms $\quad$ XV

Symbols and Acronyms xvi

1 Introduction 1

1.1 Background ......................... . . 1

1.2 Literature Survey . . . . . . . . . . . . . . . . . . . . . . . . . . 2

1.3 Motivation . . . . . . . . . . . . . . . . . . 10

1.4 Contributions . . . . . . . . . . . . . . . . . 11

1.5 Organization . . . . . . . . . . . . . . . . . 12

2 Preliminaries $\quad 14$

2.1 Bond Graph Fundamentals . . . . . . . . . . . . . . . . . . . . . . . . 14

2.1.1 Bond Graph Notation . . . . . . . . . . . . . . . . 15 
2.1.2 Basic Bond Graph Elements . . . . . . . . . . . . . . . . . 18

2.1.3 Causality for Bond Graph Elements . . . . . . . . . . . . . 28

2.2 Causal Paths . . . . . . . . . . . . . . . . . . . 37

2.3 A Rank Property of the Bond Graph . . . . . . . . . . . . . . . . . . 39

2.4 Fault Description . . . . . . . . . . . . . . . . . . . 42

3 Sensor Placement for Fault Isolability Using Low Complexity Dy$\begin{array}{ll}\text { namic Programming } & 44\end{array}$

3.1 System Description . . . . . . . . . . . . . . . . . . . 45

3.1 .1 System . . . . . . . . . . . . . . . . 46

3.1.2 Sensor Signature Matrix and Fault Signature Matrix . . . . . 46

3.1.3 Concepts for Fault Detectability and Isolability . . . . . . . . 47

3.2 Problem Formulation . . . . . . . . . . . . . . . . . . . . . . . . . . . 49

3.3 Dynamic Programming for the Optimal MI Set . . . . . . . . . 52

3.3.1 Dynamic Programming . . . . . . . . . . . . . 53

3.3.2 Low Complexity Dynamic Programming (LCDP) . . . . . . 55

3.3.3 Optimal Solutions by LCDP . . . . . . . . . . . . 57

3.4 Sensor Placement in System $\Sigma \ldots \ldots$. . . . . . . . . . . . 60

3.5 Case Studies . . . . . . . . . . . . . . . . . . . . . . 62

3.5.1 A Two-tank System . . . . . . . . . . . . . 63

3.5.2 A CyCab Front Steering System . . . . . . . . . . . . 65

3.5.3 A Truck Diesel Engine System . . . . . . . . . . . . . . . . 69

3.5.4 The Comparison Between the SPLCDP and the BILP . . . . . 71

3.6 Summary . . . . . . . . . . . . . . . . . . . 73

4 Sensor Placement for Intrinsic Fault Isolability on the Bond Graph 75

4.1 Faults and Sensor Locations on the Bond Graph . . . . . . . . . . . . 76

4.2 Motivation for Use of Casual Paths . . . . . . . . . . . . . . . . 77 
4.3 Causal Path Analysis . . . . . . . . . . . . . . . . . . 81

4.3.1 Adjacently Connected Algebraic Loop . . . . . . . . . . . . . 81

4.3.2 Assignment Rules of Causal Path for a Fault . . . . . . . . . . 84

4.4 Detectability and Distinguishability Analysis . . . . . . . . . . . . 87

4.4 .1 A DAEs Model . . . . . . . . . . . . . . . 87

4.4.2 Basic Properties of Detectability and Distinguishability . . . . 89

4.5 Sensor Placement Analysis . . . . . . . . . . . . . . . . . . . . . 94

4.5.1 Sensor Placement for Detectability . . . . . . . . . . . 95

4.5.2 Sensor Placement for Isolability . . . . . . . . . . . . . . . 99

4.5.3 Sensor Placement for Both Detectability and Isolability . . . . 103

4.6 Case Studies . . . . . . . . . . . . . . . . . . . . . . . . . 104

4.6.1 An Circuit Example with the Alternative Casual Path Config-

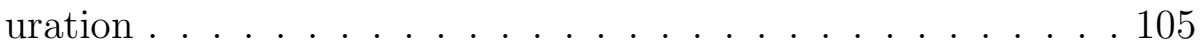

4.6.2 A Mechatronic System . . . . . . . . . . . . . 108

4.7 Summary . . . . . . . . . . . . . . . . . . . 110

5 An Efficient Method for Placing Sensor on the Bond Graph 112

5.1 A Brief Summary of Placing Sensors on the Bond Graph . . . . . . . 113

5.1.1 A Brief Statement of Two Assignment Rules . . . . . . . . . . 114

5.1.2 A Brief Statement of the Linear DAEs Model . . . . . . . 115

5.2 Problem Formulation for Sensor Placement . . . . . . . . . . . . . 116

5.2.1 Necessary and Sufficient Conditions for Fault Detectability . . 116

5.2 .2 Optimization Problem . . . . . . . . . . . . . . . . 124

5.3 Dynamic Programming . . . . . . . . . . . . . . . . . . 127

5.4 Case Studies . . . . . . . . . . . . . . . . . . . . . . . . . . 129

5.4.1 A Two-tank System . . . . . . . . . . . . . . . 129

5.4 .2 Two Other Case Studies . . . . . . . . . . . . . . . . . 134

5.5 Summary . . . . . . . . . . . . . . . . . . 137 
6 Conclusions and Recommendations

6.1 Conclusions . . . . . . . . . . . . . . . . . . . 139

6.2 Recommendations for Future Works . . . . . . . . . . . . . . . 141

$\begin{array}{lr}\text { Author's Publications } & 147\end{array}$

Bibliography

Appendix 


\section{List of Figures}

2.1 A motor-disk system . . . . . . . . . . . . . . . 15

2.2 Word bond graph for the motor-disk system . . . . . . . . . . . 15

2.3 A simple $\mathrm{BG} \ldots \ldots \ldots \ldots \ldots \ldots$

2.4 A general BG symbol for the passive 1-port element . . . . . . . . . 18

2.5 The BG symbol for an electrical resistor . . . . . . . . . . . . . 19

2.6 The BG symbol for a rotating disk . . . . . . . . . . . . . . . . . . . 19

2.7 The BG symbol for a tank . . . . . . . . . . . . . 20

2.8 A general BG symbol for modulated $R, I, C$ elements . . . . . . . . 21

2.9 The BG symbol for the active 1-port element . . . . . . . . . . . . . 22

2.10 A BG symbol for a transformer . . . . . . . . . . . . . . 23

2.11 The ideal rigid lever . . . . . . . . . . . . . . . . . 23

2.12 A BG symbol for a gyrator . . . . . . . . . . . . . . . 24

2.13 The voice coil transducer . . . . . . . . . . . . . . . . . . . 24

2.14 BG symbols for a 1 junction and a $\mathbf{0}$ junction $\ldots \ldots . . \ldots 25$

2.15 A BG example for the 1-junction . . . . . . . . . . . . . 27

2.16 BG symbols for effort and flow sensors . . . . . . . . . . . . . 28

2.17 Causalities for sources . . . . . . . . . . . . . . . . . . . 29

2.18 Possible causalities of a resistance . . . . . . . . . . . . . . . 29

2.19 Possible causalities of an inertia . . . . . . . . . . . . . . . 30

2.20 Possible causalities of a capacitor . . . . . . . . . . . . . . 30 
2.21 Possible causalities of a transformer . . . . . . . . . . . . . . . . 31

2.22 Possible causalities of a gyrator . . . . . . . . . . . . . . . 32

2.23 Causalities of $\mathbf{0}$ - and $\mathbf{1}$ - junctions $\ldots \ldots \ldots$

2.24 An example of RLC circuit . . . . . . . . . . . . . . . . . . 36

2.25 Causality assignment and complete augmentation of a bond graph for the RLC circuit . . . . . . . . . . . . . . . . . . . . . . . . 37

2.26 Four possible causalities between BG elements $\alpha$ and $\beta \ldots . . . . .37$

2.27 An example of the causal path application . . . . . . . . . . 38

3.1 A graphical model with respect to an isolating set $\mathcal{X} \ldots . . . .53$

3.2 A two-tank system with three candidate sensors. . . . . . . . . . . . 63

3.3 The CyCab front steering system. . . . . . . . . . . . . . 66

3.4 Schematic of the truck diesel engine system. . . . . . . . . . . . . . 69

4.1 An circuit example . . . . . . . . . . . . . . . . . . . 78

4.2 The DCBG of the circuit example . . . . . . . . . . . . . . . . 80

4.3 ACALs where two junctions are connected by a bond. . . . . . . . . . 82

4.4 Two examples of ACALs . . . . . . . . . . . . . . . . . . . . 82

4.5 Two other general BG diagram structures of ACDALs . . . . . . . . . 84

4.6 The DCBG with six causal paths. . . . . . . . . . . . . . . 87

4.7 The alternative DCBG with six causal paths and three sensors . . . . 105

4.8 The mechatronic system: A DC-motor interfaced with voltage divider. 109

4.9 The DCBG of mechatronic system with three causal paths and two sensors. . . . . . . . . . . . . . . . . . . 109

5.1 A two-tank system . . . . . . . . . . . . . . . . . . . 129

5.2 The DCBG of the two-tank system with four causal paths. . . . . . . 131

5.3 The DCBG of the two-tank system with three sensors . . . . . . . . 133 


\section{List of Tables}

2.1 Efforts and flows in diverse domains . . . . . . . . . . . . . . . . 16

2.2 Constitutive relations in the Laplace domain for all generic BG elements 34

3.1 The transpose of FSM for the two-tank system . . . . . . . . . . . . . 64

3.2 The transpose of SSM for the two-tank system . . . . . . . . . . . . . 64

3.3 The FSM for an optimal set $\mathcal{X}^{*}$ of ARRs in the two-tank system . . . 65

3.4 Candidate sensors in the CyCab front steering system . . . . . . . . 66

3.5 Nominal physical parameters . . . . . . . . . . . . . . . . 67

3.6 Faults in the CyCab front steering system . . . . . . . . . . 68

3.7 Eight optimal sensor sets $\mathcal{S}_{Q_{2}}{ }^{\prime} s \in \mathbb{S}_{Q_{2}}$ for the front steering system . . 68

3.8 The FSM for an optimal MI set $\mathcal{X}^{*}$ in the CyCab front steering system 69

3.9 Four system faults of the truck diesel engine system . . . . . . . . . . 70

3.10 Eight candidate sensors in the truck diesel engine system . . . . . . . 70

3.11 Five optimal sensor sets $\mathcal{S}_{Q_{2}}{ }^{\prime} s \in \mathbb{S}_{Q_{2}}$ for the truck diesel engine system 70

3.12 The FSM for an optimal MI set $\mathcal{X}^{*}$ in the truck diesel engine system 71

3.13 The comparison between the SPLCDP and the BILP . . . . . . . . . 72

4.1 The FSM of the circuit example. . . . . . . . . . . . . 107

4.2 The FSM of the mechatronic system . . . . . . . . . . . . . 110

5.1 A distinguishable matrix $\mathcal{D}$ of the two-tank system . . . . . . . . . 132

5.2 The FSM of this two-tank system . . . . . . . . . . . . . . . . 134 
5.3 The distinguishable matrix $\mathcal{D}$ of the circuit presented in Section 4.6 . 136

5.4 The distinguishable matrix $\mathcal{D}$ of the mechatronic system presented in Section $4.6 \ldots \ldots \ldots \ldots \ldots \ldots$

5.5 The comparison between the SP-DP-BG and the SP-BG . . . . . 137

A.1 Functions used in the front steering model . . . . . . . . . . . . . . 158 


\section{List of Algorithms}

$3.1 \mathbb{X}^{*}=\operatorname{LCDP}\left(\mathcal{R}, \mathcal{S}, \mathcal{F}^{\prime}\right) \ldots \ldots \ldots \ldots \ldots$

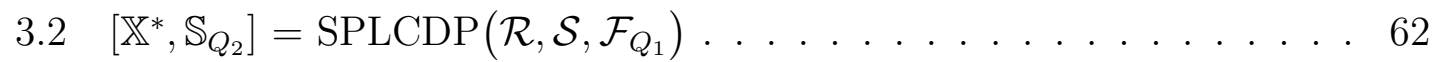

$4.1\left[\delta, \mathcal{P}^{\prime}\right]=\operatorname{DetectabilityAnalysis}\left(H(s), F_{i}(s), \mathcal{P}_{i}\right) \ldots \ldots . \ldots . \ldots 92$

$4.2 \delta=$ DistinguishabilityAnalysis $\left(H(s), F_{i}(s), F_{j}(s), \mathcal{P}_{i}, \mathcal{P}_{j}\right) \ldots \ldots . \quad 95$

$4.3 \mathbb{D}=\operatorname{Detectability}(M, \mathcal{F}, \mathbb{P}) \ldots \ldots \ldots 7$

$4.4 \xi=$ PlaceSensorUnderDetectability $(M, \mathcal{F}, \mathbb{P}) \ldots \ldots . \ldots 102$

$4.5 \xi^{*}=\operatorname{SP}-\mathrm{BG}(M, \mathcal{F}, \mathbb{P}) \ldots \ldots \ldots \ldots$

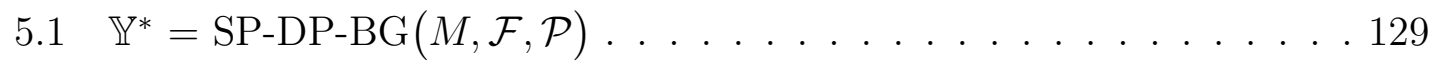




\section{Symbols and Acronyms}

$\mathcal{F}=\left\{f^{1}, f^{2}, \ldots, f^{Q}\right\}$ The set of $Q$ possible faults of concern.

$\mathcal{S}=\left\{s_{1}, s_{2}, \ldots, s_{K}\right\} \quad$ The set of $K$ candidate sensors.

FDI Fault detection and isolation.

ARRs Analytical redundancy relations

$\mathcal{R}=\left\{r_{1}, r_{2}, \ldots, r_{N}\right\} \quad$ The set of $N$ ARRs.

SSM Sensor signature matrix.

$B \quad$ The sensor signature matrix $(\mathrm{SSM})$ with respect to $\mathcal{R}$ and $\mathcal{S}$.

$S_{r_{n}} \quad$ A subset of candidate sensors in $\mathcal{S}$ embedded in the ARR $r_{n}$.

$\mathcal{X} \quad$ A subset of ARRs in $\mathcal{R}$.

FSM Fault signature matrix.

$C^{\mathcal{X}} \quad$ The fault signature matrix $(\mathrm{FSM})$ with respect to $\mathcal{X}$ and $\mathcal{F}$.

$A_{q}^{\mathcal{X}} \quad$ A fault associated $(\mathrm{FA})$ set in $\mathcal{X}$ with respect to $f^{q} \in \mathcal{F}$.

$D_{q, q^{\prime}}^{\mathcal{X}} \quad$ A fault distinguishable $(\mathrm{FD})$ set in $\mathcal{X}$ with respect to a pair of faults $f^{q}, f^{q^{\prime}} \in \mathcal{F}, f^{q} \neq f^{q^{\prime}}$.

$\mathbb{I}_{\mathcal{X}} \quad$ The set of all isolating sets. 
$\mathbb{M}_{\mathcal{X}}$

$\mathcal{X}^{*}$

$\mathbb{X}^{*}$

DP

LCDP

I

$\mathcal{X}_{u}^{i}$

$\mathbb{X}_{u}^{i}$

$\mathbb{X}^{D P}[i][u]$

$\mathbb{V}[i][u]$

$G[i]$

$\overrightarrow{1}$

$\overrightarrow{\mathbf{1}}_{m}$
The set of all minimal isolating (MI) sets.

An optimal MI set of $\mathcal{R}$.

The set of all $\mathcal{X}^{* \prime} s$.

Dynamic programming

Low complexity dynamic programming

The number of subproblems of DP used in the formulated optimization problem.

A binary vector of size $\frac{Q^{\prime 2}-Q^{\prime}}{2}$ to represent the extent to $\frac{Q^{\prime 2}-Q^{\prime}}{2}$ FD sets included by $\mathcal{X}$, which is called a state vector.

A subset of $i$ ARRs that possesses the state vector $u$.

The set of all possible $\mathcal{X}_{u}^{i \prime} s$.

The set of all $\mathcal{X}^{\prime} s$ (which contain no more than $i$ ARRs) that possess the state vector $u$ and have the least number of related candidate sensors in the DP.

The set of all $\mathcal{X}^{\prime} s$ (which contain no more than $i$ ARRs), where each possesses the state vector $u$ and relates to no more than $r$ candidate sensors in the LCDP.

The set of state vectors $u^{\prime} s$ that are updated in the $i$-th subproblem.

The vector with all one-entries.

The vector with the maximum number of one-entries. 
BILP Binary integer linear programming.

BG Bond graph.

DCBG Differentially causalled bond graph.

ACAL Adjacently connected algebraic loop.

ACDAL Adjacently connected differential algebraic loop.

DAEs Differential-algebraic equations.

$f_{i} \quad$ A flow numbered $i$.

$e_{i} \quad$ An effort numbered $i$.

D The family of detectability sets.

$\mathfrak{D}\left(f^{i}\right) \quad$ The detectability set with respect to fault $f^{i}$.

$\mathcal{J} \quad$ The set of $K$ junctions.

$\mathcal{J}_{i} \quad$ The subset of junctions of $\mathcal{J}$ with respect to fault $f^{i}$.

$\Theta_{\mathrm{sb}} \quad$ A sensor binary vector.

$y_{k} \quad$ A sensor variable.

$\mathcal{P} \quad$ The set of $Q$ sensor variables.

$\mathcal{P}_{i} \quad$ The set of sensor variables with respect to fault $f^{i}$.

$\mathbb{P} \quad$ The family of sets $\mathcal{P}_{i}$.

$\xi^{*} \quad$ The family of optimal sets of junctions.

$\mathbb{Y} \quad$ The set of all pairs of sensor variables. 
$\mathbb{Y}_{i, j}$

$\mathcal{D}_{i, j}$

$\mathcal{D}$

ISSV

MISSV

$\mathbb{M}_{\mathcal{Y}}$

$\mathcal{Y}$

$\mathcal{Y}^{*}$

$\mathbb{Y}^{*}$

$s$

$\mathcal{Y}_{s}^{i}$

$\mathbb{Y}_{s}^{i}$

$\mathbb{Y}^{D P}[i][s]$

SCAP
The set of all pairs of sensor variables with respect to faults $f^{i}, f^{j}$.

The binary set in correspondence to $\mathbb{Y}_{i, j}$.

The distinguishable matrix.

An isolating set of sensor variables.

Minimal isolating set of sensor variables.

The set of minimal ISSVs.

A set of sensor variables.

An optimal set of sensor variables of $\mathcal{Y}$.

The set of all $\mathcal{Y}^{* \prime} s$.

A binary vector of size $\frac{Q^{2}-Q}{2}$ to indicate the distinguishability of all pairs of faults, which is called a state.

A subset of sensor variables of $\mathcal{P}$ which has state $s$ and is produced by uniting $i$ pairs $\left(y_{k}, y_{k^{\prime}}\right) \in \mathbb{Y}$.

The set of all possible $\mathcal{Y}_{s}^{i \prime} s$.

The set of sets $\mathcal{Y}_{s}^{i \prime} s$, each of which has the same cardinality and is associated with a state $s$.

Sequential causality assignment procedure 



\section{Chapter 1}

\section{Introduction}

\subsection{Background}

The sensor placement issue has attracted more and more attention from both academia and industry, especially owing to an increasing number of large instrumented systems for fault detection and isolation (FDI) in the last ten years [1]. Sensors are in most cases permanently fixed to process equipment and are almost always sparsely distributed throughout the system. The number of available sensor locations is usually greater than the number used. This is partly because of structural accessibility limitations, partly because of the high cost of data acquisition systems and the instrumentation weight. Due to weight and cost considerations, sensors must be deployed in an optimum way to fulfill the requirements of system identification, state estimation, FDI, and active control. Particularly, the sensor placement consequence directly influences the capability of detecting and isolating unexpected changes. If too many sensors are installed, not only the cost incurred in providing instrumentation increases but also the overall FDI performance may be degraded to some extent. A challenging task arises as to how to deploy a minimum number of sensors for better establishing an effective FDI system. In other 
words, where should the minimum number of sensors be placed to give optimum detectability and isolability of faults?

\subsection{Literature Survey}

Physical systems are often subject to unexpected changes, like component faults and variations in operating circumstances, which tend to downgrade overall system performance, such as reliability, safety and low cost operation. To maintain a high level of performance for complex process industries (such as power stations, chemical plants, steel mills, etc.) and complex vehicles (such as aircraft and spacecraft, etc.), it is critical that such changes can be promptly detected and diagnosed so that remedial actions can be taken to reconfigure the control system and accommodate the changes [2-4] or even the shutdown action is done to avert a disaster. A monitoring system used to detect faults and diagnose their locations and significance is called a fault diagnosis system or a FDI system $[5,6]$. The design of fault diagnosis system has attracted more and more attention, due mostly to a growing demand for better performance as well as for more reliability and safety of dynamic systems.

The diagnostic task consists of three sub-tasks:

- the detection of a component malfunction

- the isolation of the faulty component

- the fault identification

When the first two sub-tasks might be performed sequentially, in most fault diagnosis systems, detection is implicit in isolation. The fault detection consists of making a binary decision: either that a system works under normal conditions or that some components are varied in an undesired way [7-11]. The fault isolation sub-task is to determine which component becomes faulty during operation, i.e., to locate a fault. 
Additionally, the third sub-task, which is to identify the magnitude and stamping time of a fault, is essential for fault diagnosis to reconfigure the system to response faults $[12,13]$.

For the viewpoint of a system, faults are usually represented as additional external signals or as parameter deviations. In the first situation, the faults are called additive faults, because in the model the faults are represented by unknown inputs which enter the model equations as addends. For example, the bias of measurement is thought of as an additive fault of a sensor. In the second situation, the faults are called multiplicative faults, since the system parameters relying on the fault size are multiplied with the input and/or system state.

Consistency checking is usually accomplished by a comparison between the measurements with their estimations, which arises from the model of a system under consideration or infers from a priori data owned by a system. The differences between the measurements and the estimations are termed residuals. Each residual should be normally zero or close to zero under fault-free conditions whilst should deviate from zero under faulty conditions. For real-time monitoring, residuals are extensively employed to design a FDI system, which are indicators to show the normal or abnormal state of a monitored system.

There are an abundance of methods to cope with faulty symptoms of a system. Generally, these methods can be classified into two categories:

- data-driven methods using a priori knowledge and the data acquired from the monitored system. These data include possible symptoms of the system. Such methods [14-17] always involve knowledge-based and artificial intelligence technologies, including fuzzy logic [15], pattern recognition [16], support vector machines [17], etc.

- model-based methods using qualitative or quantitative modelling techniques. A variety of techniques are available in the literature [18-22]. 
Model-based fault diagnosis techniques [2,3] have been extensively developed and have received a considerable attention during last three decades. The qualitative model-based approaches are normally applicable when it is unavailable to establish the numerical model describing a system. It utilizes qualitative abstractions to model systems while the structure of a system is well defined. The models used in qualitative methods are relatively simple on comparing with numerical models. The sensitivity of fault diagnosis system to model errors and sensor noises may be alleviated $[21,23]$. For FDI in a qualitative model, Some well-sophisticated techniques are reported in the literature, including signal flow graphs [24], temporal causal graphs $[25,26]$. Particularly, the temporal causal graph is normally derived from a bond graph (BG) [27]. This temporal causal graph captures temporal and causal relations in the system variables which represents the dynamic behavior of a system and further it has a complete set of labels describing the meanings of the graph edges.

Quantitative model-based approaches include observer based approaches, parameter identification approaches and parity space approaches $[5,6]$. Observer techniques $[28,29]$ have received much attention in recent research. Substantial progress in control theory and computer capability has made it possible to apply observerbased fault diagnosis techniques to nonlinear and time-varying systems as well as a party of more complex systems. Parameter identification approaches [12,30] utilize the assumption that the faults of a system reflect the changes of the physical parameters such as mass, capacitor, friction, etc. Identification of the parameters can be carried out through establishing a FDI scheme [30,31]. Parity space approaches are based on inspecting the consistency on the outputs and the inputs that are closely associated with the mathematical equations. The parity equation may lead to direct redundancy, which gives the static algebraic relation amongst the sensor outputs; or it may lead to temporal redundancy, which gives the dynamic relation between 
inputs and outputs. Roughly speaking, parity equations are obtained by the direct conversion of the input-output or state-space model of a system. All of these approaches are related in some way or other and there have been many published studies [6] showing the connections.

Actually, the residual generation problem is so difficult to realize in some systems, especially when uncertainty is concerned. This is because the fault behaves in almost exactly the same way as uncertainty and indeed is a form of uncertainty. Therefore, robust fault estimation is activated to determine the occurrence of faults. It is often more appropriate to estimate or reconstruct the actual fault effect. Once each fault is estimated robustly it is then a simple matter to isolate one fault from another and this can be done very well without residual design if the fault signals are estimated [32]. Most of the literature [32,33] about fault estimation has paid attention to the effects of additive faults. The additive fault effect causes the mean of the system output signal to vary, which is easy to tackle. On the other hand, some studies of multiplicative fault estimation have published in [34,34].

Usually, the quantitative FDI system is composed of two stages:

- residual generation: the system's inputs and outputs are processed by an appropriate algorithm (i.e., a residual generator) to generate residual signals;

- residual evaluation and threshold setting: the residual is evaluated and compared with the threshold, in order to detect, isolate and identify faults.

There are a number of strategies to design residual generators. Various approaches have been introduced in either the time domain [18] or the frequency domain [35]. The core purpose of these approaches is to improve the sensitivity to faults whilst suppressing the effects of disturbances and model errors. A directional residual generator [36] is designed on linear time-invariant finite dimensional systems and allows to isolate the faults in the multidimensional residual space. In [9,37], the differential 
geometric method is employed to construct residuals. In [38], the transmission delay and control delay are considered into constructing residual generators in networked control systems. In $[39,40]$, distributed residual generators are devised to fulfill the FDI performance on a distributed system. In [41-43], parametric structured uncertainties are accounted for and thus they are integrated into the residual generation process. Owing to inevitable inaccuracies in modelling a system, robustness relative to model errors is a critical issue in the stage of residual generator [5]. Ding et al. $[28,35,44,45]$ have done significant contributions in designing a robust residual generation process.

A decision on the possible occurrence of a fault is then made by means of the comparison of the residual feature to the pre-specified threshold. Residuals are normally zeros under ideal circumstances; and they become nonzero as a consequence of fault, noise and model errors. Due to the presence of noise, evaluated residuals are non-zeros under normal conditions. A threshold logic becomes essential in the residual evaluation, including the fixed threshold reasoning and the adaptive threshold reasoning. The difficulty of using the fixed threshold reasoning is how to set the threshold. Setting the threshold too small increases the rate of false alarms [46] while choosing it too large decreases the fault detection rate. Using the adaptive threshold reasoning can realize an optimal trade-off in the sense that the fault detection rate is maximized at the fulfillment of an allowable false alarm rate.

Traditionally, a physical system is described by a state-space representation. When the complexity of the system increases, modelling of a physical system is a demanding step due to the increased modelling difficulty. A complex system usually involves multi-domain energies and complex or unpredictable dynamic structure and behavior. Due to the multidisciplinary nature of industrial systems (such as electrical, hydraulic, electrical, etc.), a unified modelling method is required for analysis and model synthesis. The BG modelling [47-53] is well suited to this requirement. The 
BG modelling provides an approach to deal with a nonlinear system which possesses a large number of subsystems and components [54]. Additionally, a BG provides a systematic and convenient tool to design FDI procedures for nonlinear systems exchanging energy in multiple domains $[55,56]$.

Much attention has been devoted to systematically design a fault diagnosis system in a pre-established set of sensors [2,12,23,44,45,57-68]. Most of approaches deal with FDI problems under the assumption of a single fault that can occur at any instant. Some other fault diagnosis approaches [69-71] handle multiple faults simultaneously. In a system with a fixed set of sensors, there may exist a problem that more sensors are included than required. Such a problem directly leads to increase the instrumentation cost. Simultaneously, the installation of more sensors may not ameliorate the fault diagnosis efficiency but even degrade it. Finding an optimum number of sensors becomes a necessary stage before constructing a FDI system for a monitored system. In sensor placement problems, of most interest are the quantity and location of deployed sensors that are two key issues to achieve the effective FDI.

Fault detectability and isolability are of fundamental importance in the analysis and synthesis of a model-based FDI system. Fault detectability (or isolability) indicates the ability of diagnosing the incidence of the faults in a system. However, a well-known phenomenon sometimes appears in the residual evaluation stage that the effect of a fault on the residual disappears or is overwhelmed by disturbances and unknown inputs, although the fault effect on the system still exists. This well-known phenomenon makes the sensor placement topic to become a little complicated. For the sake of simplification, this thesis assume that a fault always triggers all associated residuals to non-zero values when the fault is present, in other words, faults in any residual are strongly detectable. Therefore, fault detectability discussed in this thesis has two following features: 
- it is independent of the type and the size of a fault,

- it indicates that the occurrence of a fault causes persistent inconsistency of residuals.

According to two above features, fault detectability implies the strongly detectable assumption, i.e., that a fault always makes all sensitive residuals to raise an alarm.

In the literature, there exists two types of definitions for fault detectability and isolability. In the early publications of this topic, fault detectability and isolability have often been defined in terms of the performance of a set of residuals. On the contrary, in most of recent research, fault detectability and isolability are expressed in terms of the system structural properties. Basseville [72] names these two types of notions for differentiation. One is called performance-based fault detectability (or isolability) that is with explicit reference to a particular residual generator. More specially, performance-based fault detectability (or isolability) involves a residual generation mechanism. The performance-based definition captures the signature of the fault on a set of residuals $[73,74]$. The other is termed intrinsic fault detectability (or isolability) which, differently, is without any reference to a particular residual generator. Essentially, this intrinsic definition captures the signature of the fault on the system properties other than a set of residuals $[75,76]$.

Accompanying with two types of fault detectability and isolability, sensor placement approaches are naturally categorized into two groups. One group are those optimizing the number of sensors on a set of residuals. Some works $[77,78]$ use the qualitative modelling technique to expose the conflicts caused by faults and then formulates as a problem of searching the optimal set of sensors. Some other works [79-85] formulate the sensor placement issues as an optimization problem using quantitative residuals, including the observer-based residuals [85] and the parity space based residuals $[80,81,86]$. However, it is a nontrivial task to generate all possible residuals, especially when a mathematical model involves non-linear 
and dynamic equations. To overcome that problem, specific residuals are replaced by overconstrained subsystems $[79,82-84]$, which have one degree of redundancy and represent interrelationships between faults and sensors. The other group are those [87-90] analyzing fault detectability and isolability based on system properties. After analysis, all pieces of information capable for detectability and isolability are gleaned together and then formulated as an optimization problem. These approaches for sensor placement scarcely take model uncertainties and disturbances into account.

Particularly, some relatively important model-based works are emphasized in the following. In [91], sensor location for the optimal detection performance is investigated. In [89], a novel sensor placement algorithm is designed in a linear differential and algebraic equations (DAEs) model to search out the optimal set of sensors so that a user-decision fault isolability performance is achieved. In $[85,87,88]$, the sensor location problem for FDI is structurally analyzed in a reduced system, which can be visualized by an intuitive graphical model. Additionally, other related sensor placement works are briefly reported as follows. In [92,93], a minimum cost solution is sought by a pre-specified algorithm in the data-driven model, fulfilling a desired fault isolability performance. In [94], the fuzzy learning and classification technique is used to optimally place sensors for fault detection. In [95], the optimal sensor configuration is obtained by the simultaneous perturbation stochastic approximation (SPSA), for maximizing the overall sensor response and minimizing the correlation among the sensor outputs. In [96-98], the search of the optimal set of sensors is devoted to recovery of mode observability instead of fault diagnosability. 


\subsection{Motivation}

Optimal sensor placement for model-based fault detectability and isolability has been received much interest. This topic directly relates to the overall FDI performance and the incurred instrumentation cost. Most of the recent publications pay attention to continuous-variable models. Continuous-variable models consist of sets of algebraic and differential equations. They can be deduced by applying the laws of physics, chemistry, etc. to the supervised and/or controlled system. To achieve performance-based fault detectability and isolability, the majority of methods optimize the problem built on a set of residuals. Analytical redundancy relations (ARRs) are one kind of residuals in the literature $[6,58,73,99-101]$. The parity space approach is generally recognized as one important way of generating ARRs. As the sum of faults and candidate sensors grows, the number of possible ARRs exponentially increases. For a large nonlinear system, the need of a highly efficient approach becomes necessary and apparent. The proposed approach is aimed at solving the sensor placement problem with concentration on higher efficiency and the completeness of solutions. This is the main feature of the proposed approach compared with the one in $[80,81]$.

The BG modelling is a systematic modelling framework which models a system in multiple domains. On a BG with a pre-established set of sensors, an abundance of fault diagnosis techniques $[12,25,39,40,43,55,58,59,64,65,100-104]$ have been developed. Consequently, a key issue of designing an efficient and low-cost FDI system arises as to how many sensors are required for a process to guarantee a desirable degree of fault detectability and isolability. As a result, the sensor placement issue on a $B G$ has received particular attention. On a BG, the feasibility of placing sensors at junctions is constrained by causal paths which start from the faulty BG elements and end at junctions. Additionally, to avoid generating plenty of useless residuals, the sensor placement issue on a BG is discussed to fulfill intrinsic fault 
detectability and isolability. One motivation is to derive conditions which make intrinsic fault detectability and isolability to fulfill and simultaneously the restrictions of junctions to satisfy. Then, based on these conditions, the dedicated approach for sensor placement is developed to attain the optimal solution. The other motivation is to further simplify the conditions of fault detectability and isolability by exploiting the BG characteristics. The sensor placement approach is redesigned using simpler conditions for high-efficiency performance.

\subsection{Contributions}

The dissertation develops three different sensor placement algorithms in continuous systems to achieve a maximum degree of isolability. The first one is designed based on performance-based fault detectability and isolability and the two remaining ones are to optimize sensor placement issues according to intrinsic fault detectability and isolability. A maximum degree of isolability is defined in an admissible set of candidate sensors such that as many faults as possible are isolable from others. The main contributions of this dissertation are listed as follows:

i) The fulfillment of fault detectability (respectively, fault isolability) is expressed in terms of a set of ARRs. Therefore, the sensor placement issue is formulated as a problem that is to find out a desirable set of ARRs such that the number of associated candidate sensors is least. A graphical model is established to reflect relationships among fault distinguishable (FD) sets, ARRs and candidate sensors. Dynamic programming (DP) operates on the graphical model and is a powerful and useful technique to efficiently solve this optimization problem for senor placement. With the aid of DP, the large continuous system can be efficiently handled in comparison with another existing approach. 
ii) The properties of intrinsic fault detectability and isolability on a BG are exploited and then the conditions under which a single fault can be detectable and isolable are derived. On the basis of these conditions, another sensor placement algorithm is designed. In comparison with the first sensor placement algorithm, it avoids generating an abundance of ARRs and maximum isolability is expressed in the image space of a DAEs model other than a set of ARRs.

iii) The sensor placement algorithm based on intrinsic fault detectability and isolability is redesigned to perform higher efficiency. This algorithm utilizes a simpler necessity of distinguishability which is derived by further analyzing structural characteristics of a BG. In this algorithm, a DP strategy is employed to search for the optimal solutions.

\subsection{Organization}

The remaining chapters of the dissertation is organized as follows.

- Chapter 2 briefly reviews the BG modelling language, causal paths and fault description.

- Chapter 3 introduces two important concepts fault signature matrix (FSM) and sensor signature matrix (SSM) which build relationships of ARRs with faults and candidate sensors, respectively. The maximum degree of fault detectability and fault isolability are defined on FSM. An optimization problem in the minimal isolating (MI) set is formulated for sensor placement. To efficiently find out the optimal solution of the proposed optimization problem, a low complexity dynamic programming is developed.

- Chapter 4 presents a linear dynamic system modelled by the BG modelling 
technique. Definitions of intrinsic fault detectability and isolability concerning the junction restriction are made and then the conditions under which a single fault can be detectable and isolable are exploited. Using these detectability and isolability conditions, a sensor placement algorithm is devised to iteratively search the optimal solution for achieving of maximum isolability.

- Chapter 5 further investigates BG characteristics and then gives a simpler condition for detectability. Drawing inspiration from the detectability condition, a simpler necessary condition for distinguishability is derived. By gleaning all data computed by the necessary condition, a set covering problem is established. A DP strategy is adopted to solve the set covering problem.

- Chapter 6 summarizes the conclusions of the dissertation, and provides the outlines for future work. 


\section{Chapter 2}

\section{Preliminaries}

This chapter introduces BG fundamentals and causal paths that are used to model and analyze physical systems.

\subsection{Bond Graph Fundamentals}

BGs were first introduced by Henry M. Paynter at MIT \& UT Austin in April 1959 [105] and after a decade, a conceptual framework was established by him, including most of the underlying concepts [106]. Subsequently, bond graphs were further elaborated by Ronald C. Rosenberg and Dean C. Karnopp as a systematic methodology for modeling and simulation [107-109]. Bond graphs are graphical representations of physical systems and well suited to various physical domains. As a unified multi-domain modelling approach, it provides a means to model a complex system for structural and behavior analysis [110]. It provides a unified tool to systematically model the dynamic behavior of physical systems with various energy domains, such as hydraulic, electrical, mechanical, etc. Based on the energy conversation law, the BG allows to systematically derive a set of algebraic relations and state equations. These relations and/or equations become the basis of FDI and lay a foundation for the development of sensor placement approaches. 


\subsubsection{Bond Graph Notation}

A BG is a labeled di-graph that consists of edges (which are half-arrows with lines), called bonds, representing bilaterally oriented signal flow of the power variables (i.e., efforts and flows) and multiports. A multiport refers to a point of view taken in the description of the subsystems. From the physical subsystems viewpoint, ports indicate places at which subsystems can be interconnected and power can flow between subsystems. A subsystem with one or more ports is called a multiport. Following this spirit, a system with a single port is called a 1-port, and a system with two ports is called a 2-port and so forth.

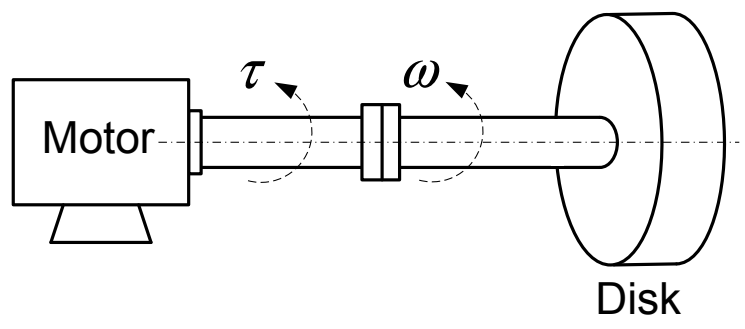

Figure 2.1: A motor-disk system

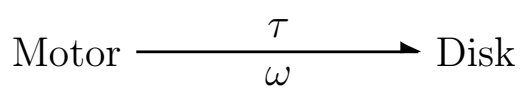

Figure 2.2: Word bond graph for the motor-disk system

A sketch of the device shown in Figure 2.1 can be treated as two multiport elements (i.e., motor and disk) and their ports can be connected with each other to form a system. Figure 2.2 depicts a word bond graph with respect to the motordisk system. The word bond graph serves to make some initial decisions about the representation of a system. Figure 2.2 implies that a port denoted by a single line emanating from the motor (labeled by the word) and another port associated with the disk have been connected together and thus a bond is formed. A pair of conjugate variables for each subsystem is constrained to be equal when two subsystems are physically linked together. For the system shown in Figure 2.2, the motor and 
Table 2.1: Efforts and flows in diverse domains

\begin{tabular}{|l|l|l|l|}
\hline \multicolumn{2}{|l|}{ Domain } & \multicolumn{1}{c|}{ Flow $(f)$} & \multicolumn{1}{c|}{ Effort $(e)$} \\
\hline Electrical & Current $(i)$ & Voltage $(e)$ \\
\hline \multirow{2}{*}{ Mechanical } & Rotation & Angular velocity $(\omega)$ & Torque $(\tau)$ \\
\cline { 2 - 4 } & Translation & Velocity $(v)$ & Force $(F)$ \\
\hline Magnetic & Flux rate $(\dot{\varphi})$ & Magnetomotive force $(M)$ \\
\hline Thermal & Entropy flow rate $(\dot{s})$ & Temperature $(T)$ \\
\hline \multirow{2}{*}{ Hydraulic } & Incompressible flow & Volume flow rate $(\dot{V})$ & Pressure $(P)$ \\
\cline { 2 - 4 } & Compressible flow & Massive flow rate $(\dot{m})$ & Enthalpy $(h)$ \\
\hline
\end{tabular}

the disk possess common coupled variables, i.e., angular speed $\omega$ and torque $\tau$. The half arrow at the end of the bond denotes the orientation of power flow that is quantified by the product of the angular velocity $\omega$ and the torque $\tau$. When the product (i.e., $\tau \omega)$ is positive, power is flowing from the motor to the disk. Similarly, power can be expressed as the product of voltage and current in the electrical domain or the product of pressure and volume flow in the hydraulic domain. Note that power variables in diverse energy domains are different but they can be classified into two types, i.e., effort and flow. In this dissertation, both the generalized notations $e$ and $f$ represent effort and flow, respectively. The product of effort and flow is the power $P=e \times f$. Table 2.1 shows effort and flow variables for several types of power interchange.

In addition to power variables (i.e., effort and flow variables), the other two types of variables turn out to be important in describing dynamic systems. These variables are called momentum $p$ and displacement $q$. The momentum is defined as the time integral of an effort, which is

$$
p=\int^{t} e d t
$$

In the same way, a displacement variable is the time integral of a flow variable:

$$
q=\int^{t} f d t
$$


The energy, E, which has passed into or out of a port is the time integral of the power, $P$. Straightforwardly,

$$
E \equiv \int^{t} P d t=\int^{t} e f d t=\int^{t} e d q=\int^{t} f d p
$$

From equation (2.3), one may find that momentum $p$ and displacement $q$ are directly associated with energy $E$. Consequently, $p$ and $q$ are also called energy variables.

To know which of the effort and flow signals at a port is the output of the multiports, BGs introduce another notion of causal stroke which is used to specify outputs. The causal stroke is a short, perpendicular line made at one end of a bond and it indicates the orientation in which the effort signal is directed. The causality is a uniquely useful feature of bond graphs which exhibits the cause and effect relationship. To summarize the above BG concepts, a concise BG notation qualifies the following four pieces of information [27]:

- a connection between two multiports by a line

- power variables $e$ and $f$ for the different types of power interchange (e.g., mechanical, electrical and so forth)

- the power direction oriented by a half arrow

- the causality using the causal stroke.

Consider an example to show four pieces of information for a BG. In Figure 2.3, $\alpha$ and $\beta$ stand for two multiports. $e$ is the effort input to $\alpha$ and $f$ is the flow output

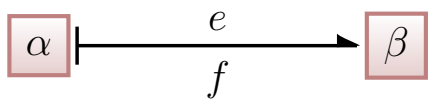

Figure 2.3: A simple BG

from $\alpha$. Conversely, $f$ is the flow input to $\beta$ and $e$ is the effort output from $\beta$. 


\subsubsection{Basic Bond Graph Elements}

With the help of the classification of power variables, BGs turn out to model a physical system in variety of energy domains using few basic types of multiports. These types of multiports are also called generic BG elements, including source elements $\{S e, S f\}$, a dissipative element $R$, storage elements $\{I, C\}$, two transducers $\{T F, G Y\}$ and two junctions $\{\mathbf{0}, \mathbf{1}\}$. According to the number of ports, generic BG elements are classified as 1-port elements $\{S e, S f, R, I, C\}$, 2-port elements $\{T F, G Y\}$ and multiport elements $\{\mathbf{0}, \mathbf{1}\}$.

\subsubsection{1-Port Elements}

A 1-port element is presented that has a single power port as well as a pair of conjugate variables $e$ and $f$ at the port. On BGs, the 1-port element is linked with a system through a single port. Some can supply power to the system, referred to as active 1-port elements (e.g., $S e, S f)$; and the rest dissipate or store power, called passive 1-port elements (e.g., $R, I, C$ ). For passive 1-port elements, the power convention is established by means of a half arrow pointing towards an element as depicted in Figure 2.4.

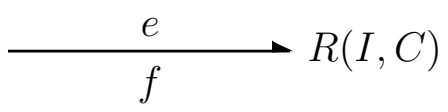

Figure 2.4: A general BG symbol for the passive 1-port element

Resistance The 1-port resistance (or resistor) can transform received power into dissipated power. In such an element, the effort and flow variables build the relationship by a static function at the single port. Figure 2.5 depicts a BG symbol of an electrical resistor. $u$ denotes the supply voltage (i.e., the effort variable) and $i$ denotes current (i.e.,the flow variable). The electrical resistor is an $R$-element governing a volt-current constitutive relation according to Ohm's law $u=R_{1} i$. As an 


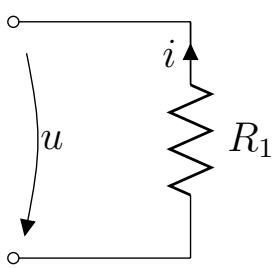

(a)

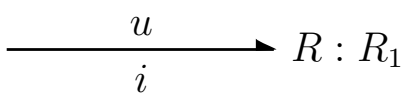

(b)

Figure 2.5: The BG symbol for an electrical resistor

analogue of the electrical domain, the damper is thought of as an $R$-element that can be characterized by a linear force-velocity relation in the mechanical domain.

Generally, the constitutive relation of an $R$-element can be expressed by

$$
\Phi_{R}(e, f)=0 \text {. }
$$

On the BG, the resistive element is a convention to show that the physical parameter is attached to the generalized name $R$ by a colon (:). Figure $2.5(\mathrm{~b})$ reveals that $R_{1}$ is the physical parameter of an electrical resistor next to the symbol $R$.

Inertia The 1-port inertia can transform the received power into kinetic energy without loss. In the device of an inertia, a momentum $p$ and a flow $f$ are related by a static constitutive law. Figure 2.6 depicts a bond graph symbol of a rotating disk in the mechanical domain. In this case, the constitutive relation of a rotating disk

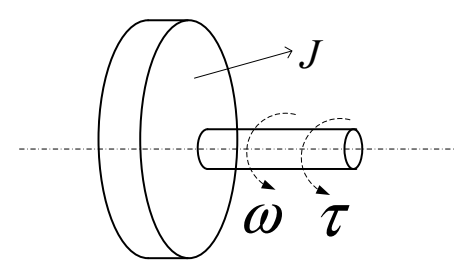

(a)

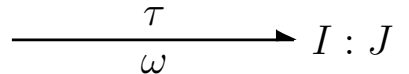

(b)

Figure 2.6: The BG symbol for a rotating disk

with moment of inertia $J$ is given by

$$
\omega=\frac{1}{J} \int^{t} \tau d t=\frac{p_{\tau}}{J}
$$


where $\omega$ is the angular velocity, $\tau$ the torque and $p_{\tau}$ the angular momentum. The inertia parameter of this case is $I=J$. In other energy domains, there exist examples of 1-port inertia elements such as an electrical inductor (electrical), mass (mechanical) and a section fluid-filled pipe (hydraulic). The constitutive relation for an $I$-element that relates the effort to the time integral of flow is

$$
\Phi_{I}\left(f, \int^{t} e d t\right)=\Phi_{I}(f, p)=0
$$

where $p=\int^{t} e d t$ is the momentum. The linear form of equation (2.6) is $e=$ $\int^{t} e d t / I=p / I$.

Capacitor The 1-port capacitor (compliance) is the other energy storing element which can transform the received power into potential energy without loss. This 1-port device reflects a static constitutive relation between a displacement $q$ and an effort $e$. Figure 2.7 shows a bond graph symbol for a tank in the hydraulic domain. $\rho$ denotes the liquid density and $A$ the cross sectional area of the tank. In this

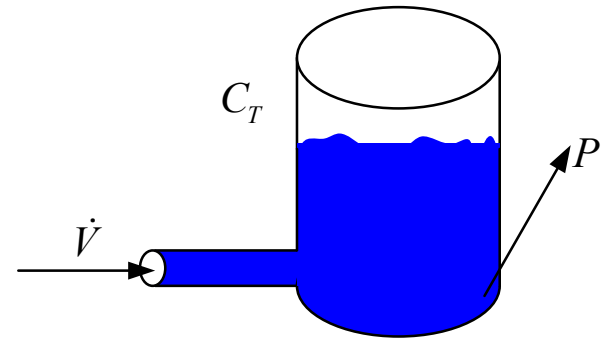

(a)

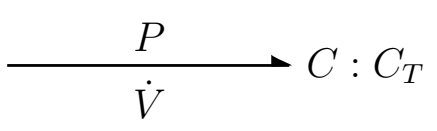

(b)

Figure 2.7: The BG symbol for a tank

example, the constitutive relation of the tank can expressed as

$$
P=\frac{1}{C_{T}} \int \dot{V} d t=\frac{\rho g}{A} V
$$


where $P$ is the pressure reading at the bottom of the tank, $\dot{V}$ the volume flow rate and $V$ the volume flowed into the tank. The compliance parameter of the tank is $C_{T}=\frac{A}{\rho g}$. Besides, the compliance phenomenon also exists in other energy domains. For instance, the electrical capacitor (in the electrical domain) and the spring (in the mechanical domain) also have compliance phenomena and thus are thought of as 1-port capacitors. In general, the 1-port capacitor can be characterized by the following constitutive relation

$$
\Phi_{C}\left(e, \int^{t} f d t\right)=\Phi_{C}(e, q)=0 \text {. }
$$

If the capacitor element can be assumed linear, then the $e$ versus $q$ curve is a straight line and the constitutive relation is of the form $e=\int^{t} f d t / C=q / C$.

It is worth noting that passive 1-port elements can be modulated by an external signal $\mu$. Considering a rheostat as the example, its resistance parameter is varied by user-decision and thus it is called a modulated resistor. The same arguments also apply to the inertia element and the capacitor element as long as their parameter values are controlled by the external signal. As a consequence, such a $C$-element is called a modulated capacitor as well as such an $I$-element is called a modulated inertia. Figure 2.8 shows a general BG symbol for passive 1-port elements.

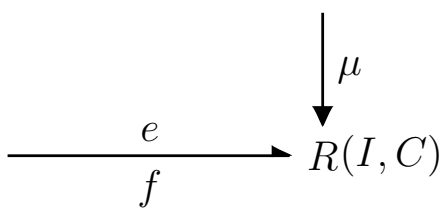

Figure 2.8: A general BG symbol for modulated $R, I, C$ elements

Source A source is regarded as an active 1-port element since it supplies power to a system at a signal port. In BG terminology, there are two types of source elements, i.e., effort source and flow source. An effort source provides effort to a 
system, which could be constant or a function of time but independent of the flow variable. Effort sources include examples such as a voltage source, a pressure pump, gravity and so on. For instance, the gravity force acting on a mass is intrinsically independent of the velocity of the mass on the earth's surface. On the other hand, a flow source imposes flow on a system which is independent of the effort variable. The flow source also can be constant or a linear or nonlinear function of time. For example, an ideal pump and an electric current generator are thought of as flow sources. Generally, source elements seem to supply power to a system, therefore a half arrow is away from the source notation and pointing towards the system connected with it. Figure 2.9 depicts the BG symbol for the active 1-port element.

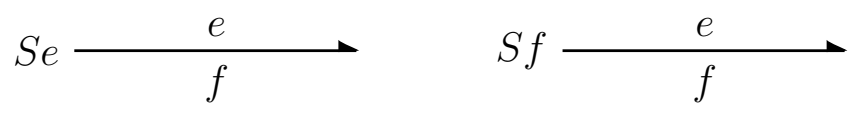

Figure 2.9: The BG symbol for the active 1-port element

\subsubsection{2-Port Elements}

The 2-port element is regarded as ideal in the sense that no energy is lost. There are two types of transducers (i.e., two basic 2-port elements): transformer and gyrator. In BG terminology, symbols $T F$ and $G Y$ denote the transformer and gyrator, respectively.

Transformer A transformer is used to make a linkage of inlet flow with outlet flow as well as a connection between inlet effort and outlet effort. In the algebraic relation of a transformer, the input flow is proportional to the output flow and the output effort is proportional to the input effort. An extremely important utility of the transformer is to cross from one energy domain to another. Figure 2.10 depicts a BG symbol of a transformer where two bonds of the transformer are numbered 1 and 2. The constitutive law for the transformer can be expressed by 


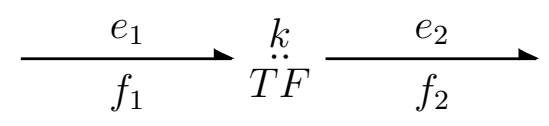

Figure 2.10: A BG symbol for a transformer

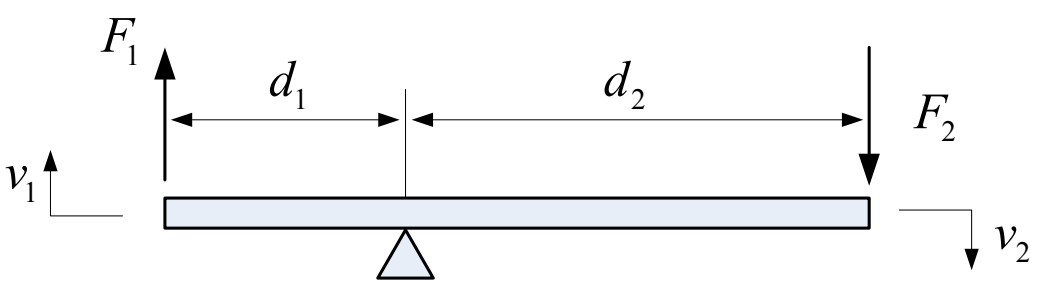

(a)

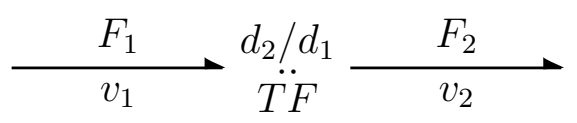

(b)

Figure 2.11: The ideal rigid lever

$$
e_{1}=k e_{2} \quad \text { and } \quad f_{2}=k f_{1}
$$

where the parameter $k$ is the transformer modulus. It is easy to see that power on the left side of a transformer is equal to that on the right side since $e_{1} \times k f_{1}=k e_{2} \times f_{2}$. The sign convention of the transformer implies that one bond points towards the transformer and the other points away from the transformer. Figure 2.11 depicts an ideal rigid level which is massless and frictionless. Such a device is a typical transformer element in the mechanical domain. In this example, $F_{1}, F_{2}$ are the forces acting on the ends of the lever and $v_{1}, v_{2}$ are the corresponding velocities. Parameters $d_{1}, d_{2}$ are the lengths. The transformer modulus of this example is $k=d_{2} / d_{1}$. Therefore, the kinematics dictates that $\left(d_{2} / d_{1}\right) v_{1}=v_{2}$ and moment equilibrium requires $F_{1}=\left(d_{2} / d_{1}\right) F_{2}$. This exactly fits the definition of a transformer in equation 2.9. Instances of transformers exist in other energy domain such as hydraulic rams (in the hydraulic domain), electrical transformers (in the electrical domain) and gears boxes (in the mechanical domain). 
Gyrator A gyrator is another 2-port BG element that has a power conserving capability. In a gyrator element, the input flow is proportional to the output effort while the output flow is proportional to the input effort. The constitutive relations of the gyrator can be expressed as

$$
e_{1}=r f_{2} \quad \text { and } \quad e_{2}=r f_{1} \text {, }
$$

where $r$ is the gyrator modulus. Figure 2.12 depicts a BG symbol for a gyrator where

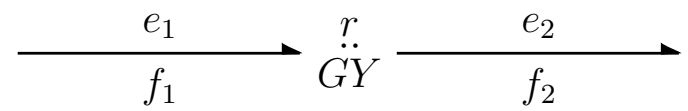

Figure 2.12: A BG symbol for a gyrator

two bonds are numbered 1 and 2. The power sign convention of a gyrator (same as the transformer) is that one bond points towards the gyrator and the other points away from the gyrator. Figure 2.13 shows a gyrator example of the voice coil. In

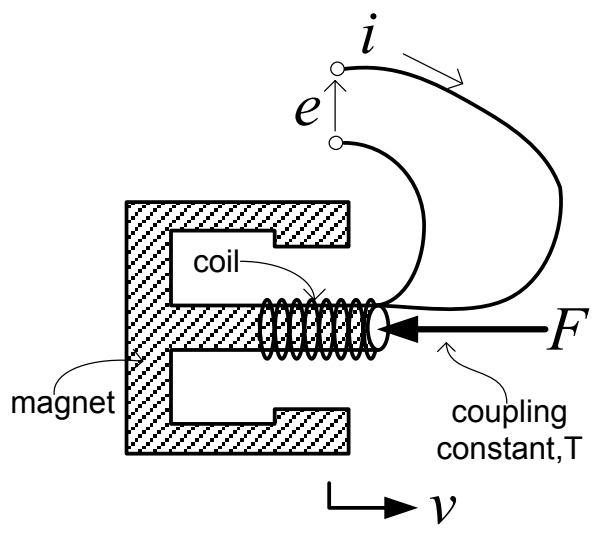

(a)

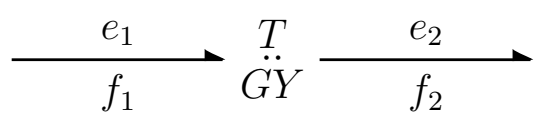

(b)

Figure 2.13: The voice coil transducer

this example, $u, i$ are the supply voltage and current; $F, v$ denote the Lorentz force and the velocity, respectively. $T$ is the coupling constant of the voice coil transducer. The voice coil transforms the power in the electric domain into that in the magnetic 


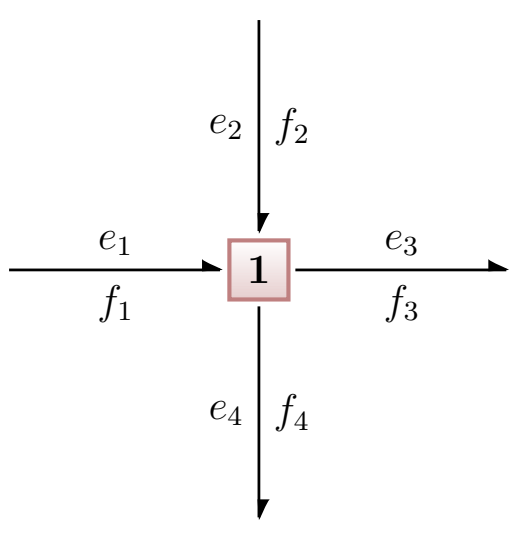

(a) 1 junction

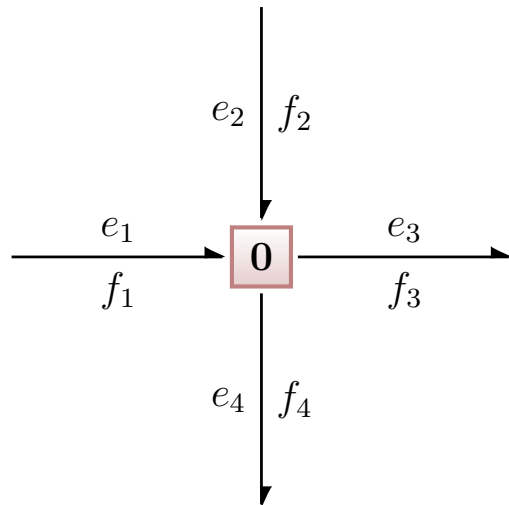

(b) 0 junction

Figure 2.14: BG symbols for a $\mathbf{1}$ junction and a $\mathbf{0}$ junction

domain. The constitutive laws of this device are

$$
u=T v \quad \text { and } \quad T i=F .
$$

Equation 2.11 reveals that the modulus of this gyrator is T. Examples for gyrators in other energy domains include gyroscopes and electrical gyrators. The modulus of a transformer or gyrator is not always constant. In some devices, the modulus changes with time or depends on other factors. Thus, the transformer with varied modulus is called a modulated transformer (MTF). Similarly, the gyrator with varied modulus is referred to as a modulated gyrator (MGY).

\subsubsection{Junctions}

Junctions serve to interconnect other multiports into subsystems or system models. Any possible connection of the 1-port or 2-port elements can be realized by two different connection patterns. These two types of connections, which, in electrical terms, are called series and parallel connections. In BG terminology, the connections are called flow and effort junctions (i.e., 1-junctions and $\mathbf{0}$-junctions). Figure 2.14 depicts BG symbols for a 1-junction and a 0 -junction. Two types of junctions are 
ideal in the sense that power is neither stored nor consumed. The constitutive law of power in the $\mathbf{1}$-junction or the $\mathbf{0}$-junction can be written as

$$
\sum_{i=1}^{n} f_{i} \times e_{i}=0
$$

where $n$ is the total number of bonds linked to the $\mathbf{1}$-junction or $\mathbf{0}$-junction. The sign of the power is taken as positive $\left({ }^{\prime}+\right.$ ') when the half-arrow points towards the junction and negative ('-') when it is oriented away from the junction. Consider the 1-junction in Figure 2.14(a). Power directions of bond 1 and bond 2 are towards this junction while those of bond 3 and bond 4 are outwards from this junction. Thus, the power relation of this junction is given by

$$
f_{1} e_{1}+f_{2} e_{2}-f_{3} e_{3}-f_{4} e_{4}=0
$$

All flows of this 1-junction are defined to be same, i.e.,

$$
f_{1}=f_{2}=f_{3}=f_{4}
$$

Solving (2.13) and (2.14) yields

$$
e_{1}+e_{2}-e_{3}-e_{4}=0
$$

In short, the constitutive law of power in the 1 -junction can be written in a simple way that the algebraic sum of the efforts is equal to zero. Similarly as for the $\mathbf{1}$-junction, the constitutive law in the $\mathbf{0}$-junction is equivalent to

$$
\left\{\begin{array}{l}
e_{1}=e_{2}=, \ldots,=e_{n} \\
\sum_{i=1}^{n} f_{i}=0
\end{array} .\right.
$$




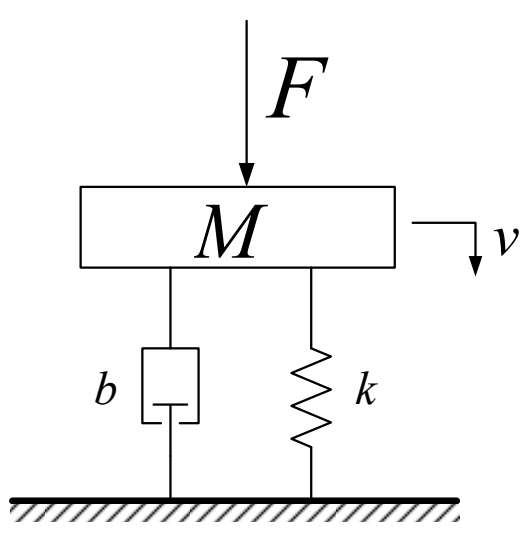

(a) A spring-mass-damper system

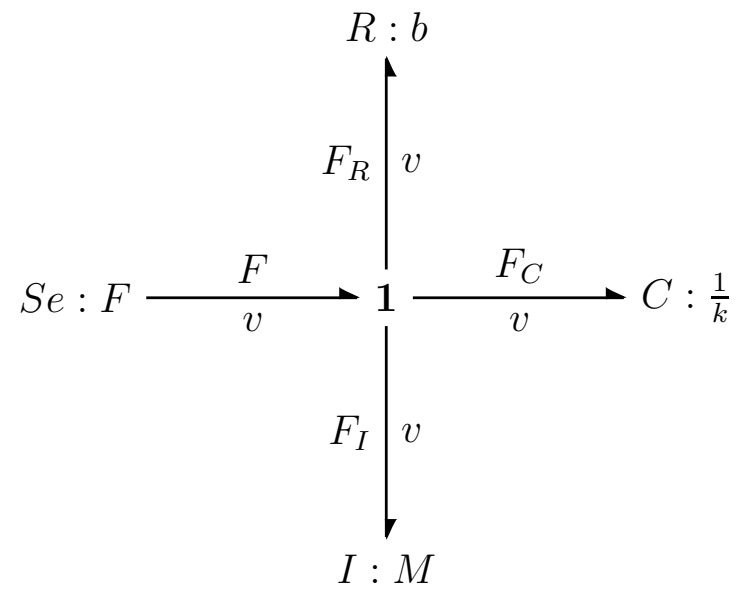

(b) The corresponding $\mathbf{1}$-junction

Figure 2.15: A BG example for the 1-junction

An example of a $\mathbf{0}$-junction is taken which is shown in Figure 2.14(b). The constitutive relations are

$$
\left\{\begin{array}{l}
e_{1}=e_{2}=e_{3}=e_{4} \\
f_{1}+f_{2}-f_{3}-f_{4}=0
\end{array} .\right.
$$

Figure 2.15 depicts the schematic of a spring-mass-damper system, which consists of a block, a damper and a spring. Symbol $M$ denotes the mass of the block, $b$ is the dumping coefficient of the damper and $k$ indicates the stiffness of the spring. Force $F$ is imposed on the block and thus velocity $v$ corresponds to the effect of force $F$ acting on the block. In Figure 2.15(b), the input force is modeled as the effort source $S e$; the damper is regarded as an $R$-element with parameter $b$; the spring is thought of as a $C$-element with $C=\frac{1}{k}$; and the block is modeled as an $I$-element with $I=M . F_{I}, F_{b}, F_{C}$ are forces acting on the block, damper and spring, respectively. A common velocity $v$ is shared by these components and simultaneously a balance of forces represents that the sum of the efforts related to this $\mathbf{1}$-junction is zero. 


\subsubsection{Sensors}

For FDI, sensors are necessary components of a system. They measure all kinds of signals of system responses like angular speed, temperature, volume flow and pressure. Sensors usually convert a non-electrical signal with a little low power into an electrical one. A distinct characteristic of sensors is that they display the readings of signals which don't affect the system's behavior. The amount of power lost from the system is so small as to be negligible. For instance, an ideal ammeter reads current without causing voltage drop; and an ideal voltmeter measures voltage whilst no current flows into it. Figure 2.16 depicts BG symbols for an effort sensor

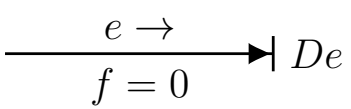

(a) An effort sensor

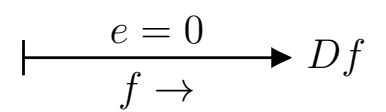

(b) A flow sensor

Figure 2.16: BG symbols for effort and flow sensors

and a flow sensor where the signal bonds are with full arrows. A causal stroke is used to denote the type of a sensor. If the causal stroke lies near the full arrow, this BG symbol denotes an effort sensor. Conversely, a flow sensor is represented by the symbol where the causal stroke is directed away from the full arrow. It is worth mentioning that the full arrow bond is known as an information bond. One of the conjugate variables with respect to an information bond is equal to zero. For instance, due to no reaction from a sensor to the system, the flow of an effort sensor is zero and the power dissipated by the effort source is zero.

\subsubsection{Causality for Bond Graph Elements}

The notion of causality is useful to indicate the directions of effort and flow on bond graphs. Some basic elements are restricted with respect to possible causalities but some are indifferent to causality. For different causalities, those basic elements 
exhibit the different forms of constitutive relations.

\subsubsection{Causality for 1-Port Elements}

Source From a causal viewpoint, the effort and flow sources are easily discussed since they have only permissible causalities. The causal stroke in Figure 2.17(a)

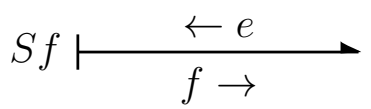

(a) Flow source

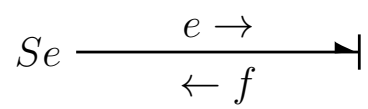

(b) Effort source

Figure 2.17: Causalities for sources

indicates the direction that the effort signal is oriented; and the causal stroke in Figure 2.17(b) is also.

Resistance As opposed to the sources, the 1-port resistance is usually indifferent to the causality imposed upon it. Figure 2.18 depicts two possible causalities for a resistance. Figure 2.18(a) indicates the conductive causality of a resistance. Under

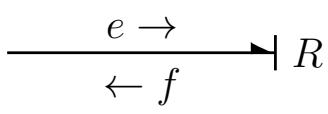

(a) Conductive causality

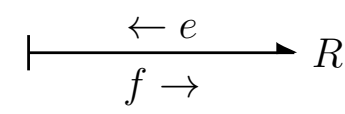

(b) Resistive causality

Figure 2.18: Possible causalities of a resistance

the conductive causality, the input of the resistance is an effort and the output is a flow. Figure 2.18(b) denotes the resistive causality of the resistance. According to the resistor causality, a flow is defined as the input while an effort is the output. These two possibilities can be expressed in equation form as follows

$$
f=\Phi_{R}^{-1}(e) \text { and } \quad e=\Phi_{R}(f),
$$

where the convection follows that the variable on the left side of the equality denotes the output of the resistor and that lying in the right side is the input variable for the 
resistor. Note that the left hand equation of (2.18) corresponds to the conductive causality and the right one is for the resistive causality.

Inertia For an inertia, there are two types of causality, namely integral causality and derivative causality. Figure 2.19 shows these two possible causalities. In integral

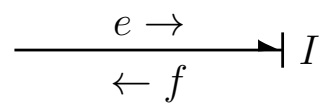

(a) Integral causality

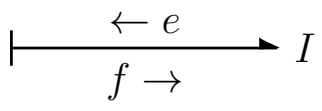

(b) Derivative causality

Figure 2.19: Possible causalities of an inertia

causality, effort $e$ is the input to an $I$-element and flow $f$ is expressed by a static function of the time integral of $e$ :

$$
f=\Phi_{I}^{-1}\left(\int^{t} e d t\right)
$$

Conversely in derivative causality, when flow $f$ is the input, effort $e$ is written as a static function of the time derivative of $f$ :

$$
e=\frac{d}{d t} \Phi_{I}(f)
$$

Capacitor Another energy-storing 1-port element, the $C$-element, is addressed here. Figure 2.20 shows two possible causalities: integral causality and derivative causality. In contrast to the $C$-element, integral causality exists when flow $f$ is the

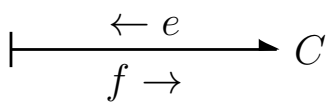

(a) Integral causality

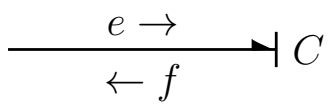

(b) Derivative causality

Figure 2.20: Possible causalities of a capacitor input to $C$-element and derivative causality exists when effort $e$ is the input. The 
capacitor relations can be expressed as follows

$$
e=\Phi_{C}^{-1}\left(\int^{t} f d t\right) \text { and } f=\frac{d}{d t} \Phi_{C}(e)
$$

Equations from (2.19) to (2.21) are written in a form suitable for nonlinear $I-$ and $C$ - elements, whilst the difference between the integral and derivative causalities remains for the special case of linear elements with their inertance and compliance parameters.

\subsubsection{Causality for 2-Port Elements}

Transformer A transformer element, denoted by $T F$, has two possible causalities as shown in Figure 2.21. Naturally, there are two kinds of constitutive relations for different possibilities. The law of Figure 2.21(a) can be expressed by

$$
f_{2}=k f_{1} \quad \text { and } \quad e_{1}=k e_{2}
$$

The other law of Figure 2.21(b) can be depicted as

$$
f_{1}=f_{2} / k \quad \text { and } \quad e_{2}=e_{1} / k
$$

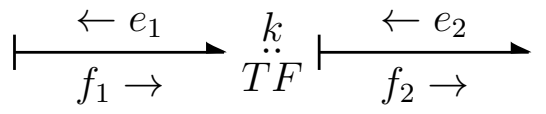

(a) One possibility

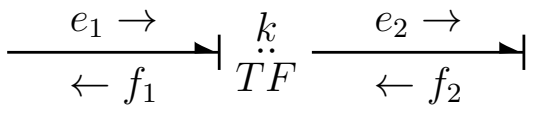

(b) The other possibility

Figure 2.21: Possible causalities of a transformer

Gyrator A gyrator is symbolized by $G Y$. Figure 2.22 depicts two possible causalities. In a 1-port gyrator, when the causality for one bond is determined, the causal- 


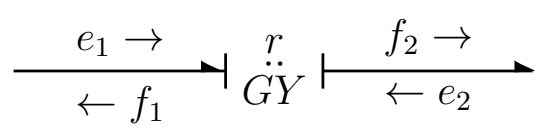

(a) One possibility

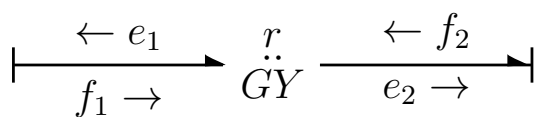

(b) The other possibility

Figure 2.22: Possible causalities of a gyrator

ity for the other is also determined. Figure 2.22(a) implies that the constitutive relations are

$$
f_{1}=e_{2} / r \text { and } f_{2}=e_{1} / r .
$$

The constitutive laws for the other possibility in Figure 2.22(b) can be expressed as

$$
e_{2}=r f_{1} \quad \text { and } \quad e_{1}=r f_{2} \text {. }
$$

\subsubsection{Causality for Junctions}

A typical permissible causality for the $\mathbf{0}$-junction is that when the effort on any single bond is specified as the input to the $\mathbf{0}$-junction, all other efforts are determined and on all other bonds they must be outputs of the $\mathbf{0}$-junction. The bond where the effort is the input to the junction is called a strong bond. The causal stroke on this bond is located near the $\mathbf{0}$-junction. Contrary to the strong bond, the remaining bonds are called weak bonds. The output of the $\mathbf{0}$-junction is the flow variable on the strong bond. Meanwhile, this flow variable on the strong bond can be expressed by the signed sum of flows of weak bonds. Figure 2.23(a) depicts the causality for the $\mathbf{0}$-junction. In this example, the strong bond specifies bond 1 and all the weak bonds are bonds 2, 3 and 4. Figure 2.23(a) shows that among four causal strokes, only one is near the $\mathbf{0}$-junction and the rest are away from the junction.

The permissible causality for the $\mathbf{1}$-junction is that if the flow on any single bond is the input to the junction, all other flows are determined and on other bonds they must be considered outputs of the junction. Similarly, the strong bond for the 1- 


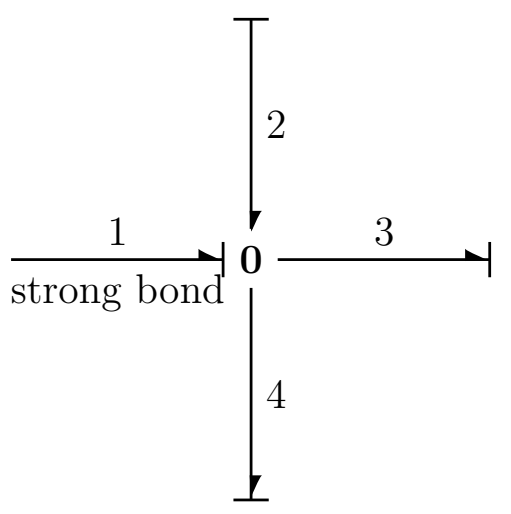

(a) $\mathbf{0}$-junction

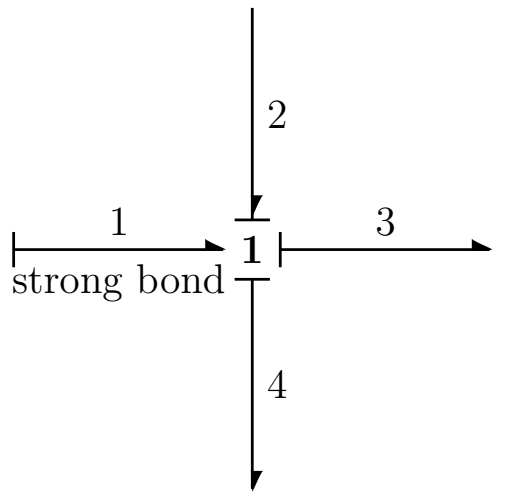

(b) 1-junction

Figure 2.23: Causalities of $\mathbf{0}$ - and $\mathbf{1}$ - junctions

junction indicates the bond where the flow is the input to the 1 -junction. All other bonds are called weak bonds in the $\mathbf{1}$-junction. The output of the $\mathbf{1}$-junction is the effort variable on the strong bond which can be expressed by the algebraic sum of efforts on other bonds. Figure 2.23(b) shows the causality for the 1-junction. In this case, the strong bond is bond 1 and weak bonds specify bonds 2,3 and 4 .

In summary, Sections 2.1.2 and 2.1.3 have introduced constitutive relations of all generic elements in the time domain. In Chapters 4 and 5, 1-port BG elements with linear behaviours will be investigated in the Laplace domain and thus constitutive relations of all generic elements in the Laplace domain are recapitulated in Table 2.2.

\subsubsection{Causality Assignment for Bond Graph}

The basic notions of causality for a variety of elements are introduced above. It is now appropriate to apply such information to the entire system in an orderly fashion. On BGs, the signal propagation follows inherent natures of various elements. When a signal (effort or flow) reaches a junction, it is either distributed (flows any one of the weak bonds if the signal is the variable of the strong bond of the junction) or goes to the strong bond (if it is a variable of the weak bond). When the signal 
Table 2.2: Constitutive relations in the Laplace domain for all generic BG elements

\begin{tabular}{|c|c|c|}
\hline Name & Symbol & Relation \\
\hline Effort source & $(M) S e: E \frac{e_{1}}{f_{1}}-1$ & $e_{1}=E$ \\
\hline Flow source & $(M) S f: F \stackrel{e_{1}}{f_{1}}$ & $f_{1}=F$ \\
\hline \multirow[t]{2}{*}{ Resistance } & $\longmapsto \frac{e_{1}}{f_{1}}-R$ & $e_{1}=R f_{1}$ \\
\hline & $\begin{array}{l}e_{1} \\
f_{1}\end{array}$ & $f_{1}=e_{1} / R$ \\
\hline \multirow[t]{2}{*}{ Capacitance } & $\begin{array}{c}e_{1} \\
f_{1}\end{array}$ - & $f_{1}=C s e_{1}{ }^{*}$ \\
\hline & $e_{1} \longrightarrow C$ & $e_{1}=\frac{1}{C s} f_{1}+e_{1}(0)^{*}$ \\
\hline \multirow[t]{2}{*}{ Inertia } & $\longmapsto \quad e_{1} \longrightarrow L$ & $e_{1}=L s f_{1}^{*}$ \\
\hline & 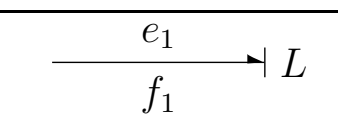 & $f_{1}=\frac{1}{L s} e_{1}+f_{1}(0)^{*}$ \\
\hline \multirow[t]{2}{*}{ Transformer } & 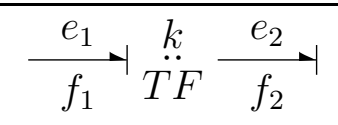 & $e_{2}=e_{1} / k, f_{1}=f_{2} / k$ \\
\hline & $\stackrel{e_{1}}{\stackrel{f_{1}}{\longmapsto}} \stackrel{\stackrel{k}{T} F}{\longmapsto} \stackrel{e_{2}}{f_{2}}$ & $e_{1}=k e_{2}, f_{2}=k f_{1}$ \\
\hline \multirow[t]{2}{*}{ Gyrator } & 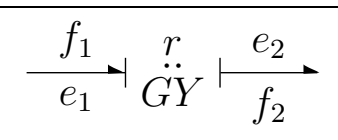 & $f_{2}=e_{1} / r, f_{1}=e_{2} / r$ \\
\hline & $\stackrel{f_{1}}{e_{1}} \stackrel{\ddot{G} Y}{\stackrel{r}{f_{2}}} \stackrel{e_{2}}{-}$ & $e_{1}=r f_{2}, e_{2}=r f_{1}$ \\
\hline 0 -junction & $\frac{e_{1}}{f_{1}}-\mathbf{0} \frac{e_{2}}{f_{3} \mid f_{3}}$ & $\begin{array}{l}f_{1}=f_{2}+f_{3}, \\
e_{1}=e_{2}=e_{3}\end{array}$ \\
\hline 1-junction & $\underset{f_{1}}{e_{3} \mid f_{3}} \stackrel{e_{2}}{\underset{f_{2}}{\longrightarrow}}$ & $\begin{array}{l}e_{1}=e_{2}+e_{3}, \\
f_{1}=f_{2}=f_{3}\end{array}$ \\
\hline
\end{tabular}

${ }^{*} s$ is the laplace variable; $f_{1}(0), e_{1}(0)$ denote initial conditions of $L$ and $C$, respectively. 
reaches a passive element $(R, I$ or $C)$ it returns back through it but undergoes a qualitative change (flow variable becomes effort variable and vice verse). When a signal reaches a source or sensor, it is terminated there. Therefore, a procedure is needed to assign causality so as to visualize explicitly and graphically how effort and flow signals' traverse on the entire BG.

A procedure, named Sequential Causality Assignment Procedure (SCAP) was developed to assign these causalities to a BG [54,110]. The procedure can be described as follows:

i. Choose any source $(S e$ or $S f)$, and assign its required causality. Immediately extend the causal implications through the graph as far as possible, using the constraint elements $(\mathbf{0}, \mathbf{1}, T F, G Y)$ which may be connected to the source. These four elements are called constraint elements because only certain causal patterns are allowed.

ii. Repeat Step i until all sources have been assigned.

iii. Choose any energy storage element $(C$ or $I)$, and assign its preferred integral causality. Immediately extend the causal implications through the graph as far as possible, using the constraint elements $(\mathbf{0}, \mathbf{1}, T F, G Y)$.

iv. Repeat Step iii until all energy storage elements have been assigned.

v. Choose any unassigned R-element and assign a causality to it (basically arbitrary). Immediately extend the causal implications through the graph as far as possible, using the constraint elements $(\mathbf{0}, \mathbf{1}, T F, G Y)$.

vi. Repeat Step v until all remaining bonds have been assigned.

vii. Choose any remaining unassigned bonds (joined to two constraint elements), and assign a causality to each of these arbitrarily. Immediately extend the 


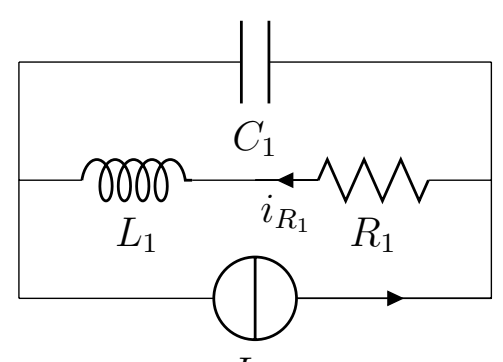

$I_{\text {in }}$

(a)

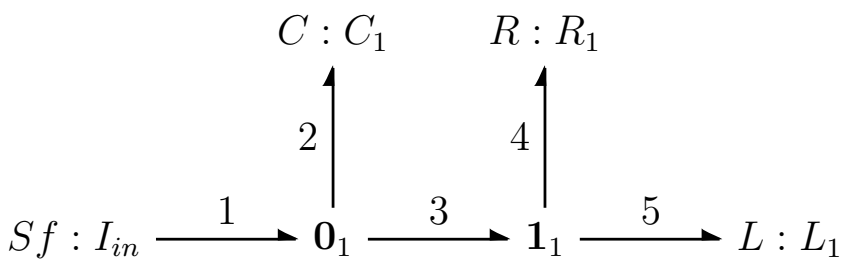

(b)

Figure 2.24: An example of RLC circuit

causal implications through the graph as far as possible, using the constraint elements $(\mathbf{0}, \mathbf{1}, T F, G Y)$.

viii. Repeat Step vii until all remaining bonds have been assigned.

Assignment of causality is carried out step by step in the example in Figure 2.24. This example circuit shown in Figure 2.24(a) consists of a current generator, an inductor, a capacitor and a resistor. For demonstration purposes, the integral causality for the energy-storing element is chosen. Using the bond graph construction procedure for circuits [110], the bond graph for this RLC circuit is given in Figure 2.24(b). In Figure 2.25(a), bond 1 is directed according to the meaning of the source element (the flow source $S f$ ). Since the strong bond for junction $\mathbf{0}_{1}$ has not been determined, the other bonds connected to $\mathbf{0}_{1}$ cannot yet be assigned a causality. There are no more source and then the SCAP proceeds to Step iii. In Figure 2.25(b), capacitor $C_{1}$ is assigned in integral causality. Immediately, bond 3 is causally directed due to junction $\mathbf{0}_{1}$ which has only one effort input, $e_{2}$. In Figure $2.25(\mathrm{c})$, inertia $I_{1}$ is put in integral causality. Immediately, bond 4 is causally directed because of junction $\mathbf{1}_{1}$ which has only one flow input, $f_{5}$. At this point, causality assignment for all bonds is finished. From Figure 2.25(c), it is not hard to see that components $I_{\text {in }}$ and $C_{1}$ are connected in parallel since they share the same effort. Furthermore, components $R_{1}$ and $I_{1}$ are connected in series due to their shared flow. 


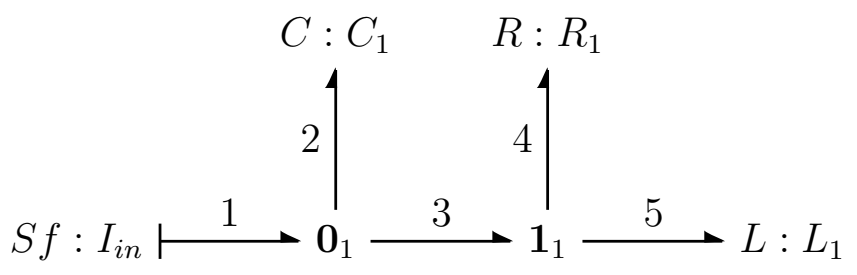

(a)

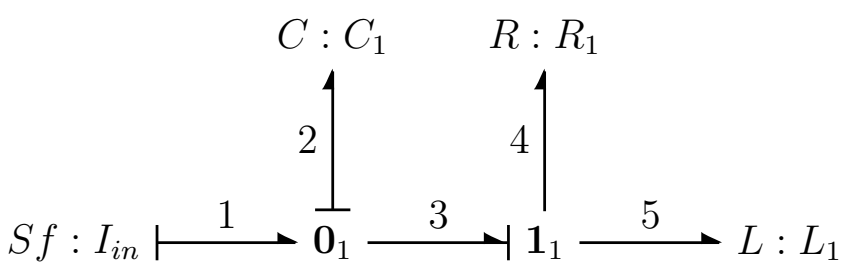

(b)

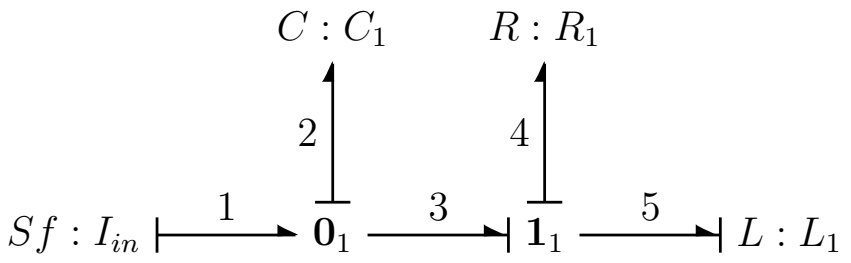

(c)

Figure 2.25: Causality assignment and complete augmentation of a bond graph for the RLC circuit

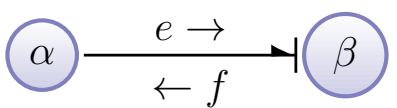

(i)

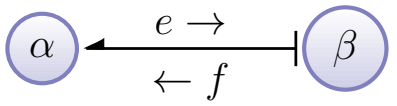

(iii)

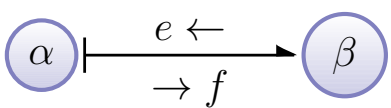

(ii)

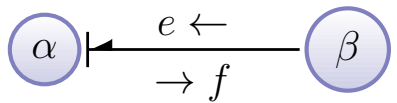

(iv)

Figure 2.26: Four possible causalities between BG elements $\alpha$ and $\beta$.

\subsection{Causal Paths}

On BGs, power flowing from one multiport to another follows the sequence of half-arrows. However, an effort signal travels along causal strokes. Note that the half-arrow sign convention for power flow and the causal stroke are totally independent. Thus, using $\alpha$ and $\beta$ to stand for BG elements, Figure 2.26 shows all the combinations of sign convention and causal strokes. A routine of a power variable 
signal that propagates from a multiport to another is called a signal transmission path. In BG terminology, a bond path between two BG elements, like $C, S e, L$, $R$ and so on, via junctions (i.e., $\mathbf{0}$ and $\mathbf{1}$ ), $T F$ and $G Y$ is called a causal path if the sequence of causal strokes qualifies that the causal strokes are towards the same direction with the exception of a path through a $G Y$ where those causal strokes' direction is always altered. For instance, in Figure 2.25(c), the causal path from the source $I_{\text {in }}$ to BG element $L_{1}$ is

$$
I_{\text {in }} \rightarrow f_{1} \rightarrow \mathbf{0}_{1} \rightarrow f_{2} \rightarrow C_{1} \rightarrow e_{2} \rightarrow \mathbf{0}_{1} \rightarrow e_{3} \rightarrow \mathbf{1}_{1} \rightarrow e_{5} \rightarrow L_{1}
$$

The stroke indicates the direction of the effort signal and the opposite direction of the flow signal. Therefore, each causal path includes the propagations of effort and flow signals.

One role of a causal path played is to graphically show the relation between system variables. This role is well suited to a large complex system where a lot of unknown variables need to be systematically expressed in terms of known variables. Figure 2.27 illustrates an application of deriving an analytical expression of unknown variable $e_{2}$. In this example, there are three components (i.e., a source

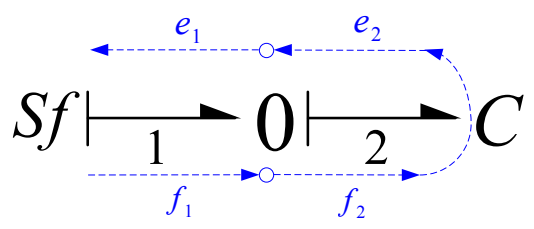

Figure 2.27: An example of the causal path application

$S f$, a capacitor $C$ and $\mathbf{0}$-junction) and the known variable is the source $S f$. The causal path between $e_{2}$ and $S f$ is given as $S f \rightarrow f_{1} \rightarrow \mathbf{0} \rightarrow f_{2} \rightarrow C \rightarrow e_{2}$. First consider a capacitor $C$ whose input is $f_{2}$ and output is $e_{2}$. This causality implies that the constitutive relation $\left(\Phi_{C}^{-1}\right)$ is $e_{2}=\Phi_{C}^{-1}\left(f_{2}\right)$. Additionally, a $\mathbf{0}$-junction connects with two bonds and bond 2 is the strong bond. Therefore, $f_{2}=f_{1}=S f$ 
and then $e_{2}=\Phi_{C}^{-1}(S f)$. This application shows that the causal path represents how unknown power variables can be calculated from know variables. Therefore, a causal path can be considered as a powerful tool to analytically and systematically derive the relation for two connected variables.

This advantage of a causal path is significant for fault diagnosis. When a component becomes faulty, its fault signal (an effort or flow signal) is delivered to other BG elements. If the signal reaches a junction that has been attached to a sensor, then the signal is monitored by this sensor. After the FDI technique deals with the monitored signal, the fault exposes. Therefore, for fault diagnosis, causal paths between junctions and possible faulty components are of much interest. Causal paths become useful tools to graphically diagnose faults on BGs. In the model-based FDI, causal paths are used as essential tools $[58,59,111,112]$ to systematically eliminate unknown system variables and thus generate primary ARRs.

\subsection{A Rank Property of the Bond Graph}

A storage element in a BG, like a $C$-element or an $I$-element, can be assigned in two types of causalities: derivative and integral. if all storage elements of a BG are in derivative (integral) causality, this BG is called in derivative (integral) causality. On the other hand, a BG is said in mixed causality, if the BG contains more than one storage elements and these elements involve both derivative and integral causalities. Summarizing the constitutive relations and causalities leads to a rank property of the BG. This rank property is presented as the following lemma.

Lemma 2.1. Assume a $B G$ with $n$ bonds contains $2 n$ conjugate power variables. Let a vector $x_{1}=\left[e_{1}, f_{1}, e_{2}, f_{2}, \cdots, e_{n}, f_{n}\right]^{\top}$ represent these $2 n$ variables. Thus, all 
constitutive relations of this $B G$ can be expressed as

$$
B(s) x_{1}=b,
$$

where $B(s)$ is a polynomial matrix of $s$ and vector $b \neq \boldsymbol{O}$. Matrix $B(s)$ describing this $B G$ either in derivative causality or in integral causality, or even in mixed causality is full column rank.

Proof. Let $\mathcal{E}_{i}$ represent one constitutive relation in a BG and $p_{\mathcal{X}}\left(\mathcal{E}_{i}\right)=\mathcal{X}_{i}$ denote the set of power variables in a relation $\mathcal{E}_{i}$. Consider a 1 -type junction with $l$ bonds as well as $2 l$ conjugate power variables (similar to a $\mathbf{0}$-type junction). There are one effort-summed relation and $\left(\begin{array}{l}l \\ 2\end{array}\right)$ flow-equalized relations. However, $l-1$ flow equalities of one strong bond with the rest $l-1$ bonds can completely represent $\left(\begin{array}{l}l \\ 2\end{array}\right)$ flow equalities in this junction. Additionally, the $l$ number of bonds linked to $l$ number of other BG elements provide $l$ number of constitutive relations. Therefore, these $2 l$ constitutive relations describe concisely the dynamics of this junction. To an $n$-bond graph, $2 n$ constitutive relations are sufficient to describe the dynamics.

In a junction with $l$ bonds, note that power variables in $\mathcal{X}_{i}$ hold only one constitutive relation (i.e., $\mathcal{E}_{i}$ ). As a result, $\mathcal{X}_{1} \neq \mathcal{X}_{2}, \ldots, \neq \mathcal{X}_{2 l}$ and

$$
\forall \mathcal{X}_{i}, \nexists\left[\alpha_{1}, \alpha_{2}, \ldots, \alpha_{i-1}, \alpha_{i+1}, \ldots, \alpha_{2 l}\right], \mathcal{X}_{i}=\sum_{k=1, k \neq i}^{2 l} \alpha_{k} \mathcal{X}_{k}
$$

where the plus-minus sign of $\alpha_{k}$ represents two different set-theoretic operations. The union is taken among sets $\mathcal{X}_{k}$ when the sign of $\alpha_{k}$ is positive and otherwise the symmetric difference is used. In addition, $\left|\alpha_{k}\right|$ denotes the coefficient of the set $\mathcal{X}_{k}$. Because $2 l$ conjugate power variables of this junction (i.e., $2 l$ power variables) do 
not appear in the rest $2 n-2 l$ constitutive relations, it can be concluded that

$$
\nexists\left[\alpha_{1}, \alpha_{2}, \ldots, \alpha_{i-1}, \alpha_{i+1}, \ldots, \alpha_{2 n}\right], \mathcal{X}_{i}=\sum_{k=1, k \neq i}^{2 n} \alpha_{k} \mathcal{X}_{k}
$$

for all $\mathcal{X}_{i}$ in these $2 l$ relations. Generally, a BG model consists of several junctions. To expand this conclusion, (2.27) still fulfills for all $\mathcal{X}_{i}$ in $2 n$ relations.

Let $\mathbb{E}=\left\{\mathcal{E}_{1}, \mathcal{E}_{2}, \ldots, \mathcal{E}_{2 n}\right\}$ denote the set of $2 n$ constitutive relations and $\mathcal{X}=$ $\bigcup_{k=1}^{2 n} \mathcal{X}_{i}$ denote the set of all power variables. The BG model can be expressed into a square matrix $B_{s}^{n}$ that crosses constitutive relations in rows and power variables in columns where an entry $c_{i j} \neq 0$ (which represents the respective coefficient of a power variables) if a power variable in $\mathcal{X}$ appears in a relation in $\mathbb{E}$ and $c_{i j}=0$ otherwise. For example, considering a constitutive relation $f_{i}=C s e_{i}$ for a capacitor element, the corresponding entry is $c_{i j}=C s$. According to (2.27), in conclusion, each row of $B_{s}^{n}$ is independent of the others. Therefore, the matrix $B_{s}^{n}$ has rank $2 n$ (i.e., full column rank). In other words, the behavioural model described by $\mathbb{E}$ and $\mathcal{X}$ is a just-determined model [2].

In a $\mathrm{BG}$ with $n$ bonds, besides $2 n$ constitutive relations stated above, there exist other equalized relations between weak bonds. However, these relations depend on the equalized relations of strong bonds with weak bonds. Note that matrix $B(s)$ in (2.26) represent all constitutive relations of the $n$-bond BG. Thus, there always exists an elementary matrix $U(s)$ such that $U(s) B(s)=\left[\begin{array}{c}B_{s}^{n}(s) \\ \mathbf{0}\end{array}\right]$. Hence matrix $B(s)$ has full column rank.

Clearly, $B(s)^{\prime} s$ in different causalities exhibit diverse forms. They can be convertible from one another. For example, the $B(s)$ in derivative causality is left-multiplied by $\operatorname{Diag}[\underbrace{1, \cdots, 1}_{2 k},-C s, \underbrace{1, \cdots, 1}_{2 n-2 k-1}]$, resulting in it being in mixed causality because the storage element linked to bond $k$ becomes in integral causality. Vector $b$ in 
derivative causality only includes inputs whilst in mixed/integral causality contains initial conditions of storage elements besides inputs.

\subsection{Fault Description}

Generally, faults are deviations from the normal behavior in the system or its instrumentation. The faults of interest belong to one of the following categories: additive faults and multiplicative faults. Additive faults are referred to as unknown inputs acting on a system, which are normally zero and which, when occur, influence the outputs independent of the known inputs. Multiplicative faults are deviations from nominal parameters and their qualities depend on the magnitude of the know inputs. This thesis introduces the faults occurring on algebraic and differential equations and the BG elements, respectively. A fault occurring a algebraic and differential equation is easy to describe. Usually, the occurrence of faults are modeled as addends entering the algebraic and differential equations. Take the capacitor in Figure 2.24(a) for example. The voltage $U_{1}$ and the current $I_{\text {in }}$ of the capacitor $C_{1}$ are of the following form

$$
I_{\text {in }}=C_{1} \frac{d}{d t} U_{1}
$$

If the capacitor $C_{1}$ is faulty, the change $f^{1}$ is directly added to the parameter $C_{1}$, seeing the following equation

$$
I_{i n}=\left(C_{1}+f^{1}\right) \frac{d}{d t} U_{1}
$$

On a BG, depicting its faulty elements is important for sensor placement. A good understanding of the relationship between a real fault and its effect on the mathematical model is a prerequisite. An actuator is thought of as a source element to a system and thus its fault directly imposes an additional power variable on the 
system, for instance, in case of an effort source

$$
E=e_{i}+\Delta_{i}
$$

where $\Delta_{i}$ is the additional effort variable. To other 1-port elements, their faults $f^{i \prime} s$ are modeled as additional parameter values. These are referred to as multiplicative faults. For instance, an inertia $L$ in derivative causality with the following constitutive relation

$$
e_{i}=L s f_{i}
$$

If the element $L$ is faulty, then an additional value $\Delta L$ is added to the normal value $L$ and (2.31) is rewritten by

$$
e_{i}=(L+\triangle L) s f_{i}=L s f_{i}+\Delta L s f_{i}=L s f_{i}+\Delta_{i}
$$

where $\Delta_{i}$ is a fault signal (i.e., an additional power variable) and indicates the bias of an effort variable $e_{i}$. Additionally, the non-zero fault signal $\Delta_{i}$ represents the occurrence of fault $f^{L}$. It is worth noting that in Chapters 4 and 5 either an additive fault or a multiplicative fault is represented by an addition of the power variable. 


\section{Chapter 3}

\section{Sensor Placement for Fault}

\section{Isolability Using Low Complexity Dynamic Programming}

This chapter tackles the sensor placement problem in a system to find the optimal set of candidate sensors for fault detectability and isolability. Because the optimal set of candidate sensors for the sensor placement problem completely rests on this optimal minimal isolating (MI) set, the proposed method concentrates on determining the optimal MI set from the set of all ARRs. For the search of the optimal MI set, a dynamic programming (DP) strategy is developed in a graphical model which interprets graphically the relationships among fault distinguishable (FD) sets, ARRs and candidate sensors. DP inherently qualifies an out-performance on searching solutions from large data. To more efficiently solve the optimization problem and faster reach the optimal MI set, this chapter develops a low complexity dynamic programming (LCDP) that qualifies lower computational complexity and space complexity than DP. Generally, a system considers two types of faults (i.e., system faults and sensor faults) for isolability. Sensor faults dynamically vary 
with different candidate sensors chosen. The exhaustive way $[80,81,113]$ copes with all faults of candidate sensors. Nevertheless, a lot of space needs for storing data and the efficiency is pretty low since a portion of time wastes on isolating faults of unused candidate sensors. As to this issue, this chapter proposes a dedicated procedure to determine sensor faults, thereby improving the efficiency and saving the storage space. Finally, the proposed sensor placement approach applies on case studies and performs better than an existing sensor placement approach in terms of efficiency.

The organization of this chapter is as follows. Section 3.1 describes the system under consideration and then introduces the basic concepts for fault detectability and isolability. Section 3.2 formulates the sensor placement problem with respect to a set of ARRs and Section 3.3 gives dynamic programming algorithms to seek the optimal MI set. Section 3.4 develops a sensor placement algorithm based on LCDP for isolability of system faults and sensor faults. Section 3.5 illustrates the application of the proposed approach on the two-tanks system and applies the approach to the CyCab front steering system and the truck diesel engine system. Then, in the latter two systems, the performance of the approach compares with that of an existing sensor placement method for fault detectability and isolability. Finally, Section 3.6 summarizes the work and contributions of this chapter.

\subsection{System Description}

The system under consideration is presented with possible faults and candidate sensors. Some fundamental concepts are introduced to formulate constraints imposed on the sensor placement problem. 


\subsubsection{System}

Consider a system denoted by $\Sigma(\mathcal{S}, \mathcal{F})$. The system $\Sigma$ consists of multiple components and subsystems integrated by signal follows and energy transfers. $\mathcal{S}=$ $\left\{s_{1}, s_{2}, \ldots, s_{K}\right\}$ denotes the set of $K$ candidate sensors that are possibly installed with no repetition. $\mathcal{F}=\left\{f^{1}, f^{2}, \ldots, f^{Q}\right\}$ represents the set of $Q$ possible faults of concern. All placed sensors can be faulty and thus are considered among the possible faults of concern. The $Q_{1}$ possible system faults are critical faults of concern. The $Q_{2}$ possible sensor faults refer to the $Q_{2}$ placed sensors which may be faulty during operation. Before an optimal set of candidate sensors is determined, $Q_{2}$ is unknown and hence so is $Q=Q_{1}+Q_{2}$. For convenience of description, the fault set $\mathcal{F}$ is divided into two parts, $\mathcal{F}_{Q_{1}}$ containing $Q_{1}$ possible system faults and $\mathcal{F}_{Q_{2}} Q_{2}$ possible sensor faults.

In model-based FDI, ARRs are used to indicate the normal state or abnormal state of system $\Sigma$ operation. ARRs arise from system equations of the system $\Sigma$ and are functions of measurable variables and fault signals. Systematic derivations of ARRs from system equations, e.g., [79,82], have been extensively studied in modelbased FDI. For the considered system $\Sigma, \mathcal{R}=\left\{r_{1}, r_{2}, \ldots, r_{N}\right\}$ is defined as the set of all ARRs taking all candidate sensors as present.

ARRs are expressed in terms of measurements and thus relate to candidate sensors. Exploiting this property of ARRs, this chapter determines the optimal sensor set for fault detectability and isolability by finding a set of ARRs involving the least number of candidate sensors. This chapter assumes that one fault occurs at a particular time.

\subsubsection{Sensor Signature Matrix and Fault Signature Matrix}

The relationships between all ARRs and all sensors can be summarized in the sensor signature matrix (SSM) defined as $B \in\{0,1\}^{N \times K}$ with $b_{n k}=1$ if the can- 
didate sensor $s_{k} \in \mathcal{S}$ is embedded in the ARR $r_{n} \in \mathcal{R}$ and $b_{n k}=0$ otherwise. A row of SSM $B$ defines the relationship of an ARR with some embedded candidate sensors. The set of candidate sensors embedded in the ARR $r_{n}$ is defined as $S_{r_{n}}=\left\{s_{k} \in \mathcal{S}: b_{n k}=1\right\}$.

To a subset of ARRs $\mathcal{X} \subseteq \mathcal{R}$, the relationship with a set of faults $\mathcal{F}$ can be depicted by a fault signature matrix $(\mathrm{FSM}) C^{\mathcal{X}} \in\{0,1\}^{|\mathcal{X}| \times|\mathcal{F}|}$ such that $c_{n q}^{\mathcal{X}}=1$ if the ARR $r_{n} \in \mathcal{X}$ is affected by the fault $f^{q} \in \mathcal{F}$ (if it occurs) and $c_{n q}=0$ otherwise. The columns of the FSM $C^{\mathcal{X}}$ are called consistency vectors [58,102,114] (or fault code [11]) and are used to define the detectability/isolability of a fault.

\subsubsection{Concepts for Fault Detectability and Isolability}

In the sensor placement problem, the optimal set of ARRs is subject to the fault isolability constraint. This constraint can be formulated into a particular set of ARRs in the following context. Because fault isolability is based on fault detectability, first of all, it is necessary to express fault detectability in terms of a set of ARRs. Accordingly, the following definition of fault associated sets is introduced.

Definition 3.1. Given the FSM $C^{\mathcal{X}}$, the fault associated (FA) set $A_{q}^{\mathcal{X}}$ in $\mathcal{X}$ with respect to a fault $f^{q} \in \mathcal{F}$ is defined as $A_{q}^{\mathcal{X}}=\left\{r_{n} \in \mathcal{X}: c_{n q}^{\mathcal{X}}=1\right\}$.

If the FA set $A_{q}^{\mathcal{R}}$ of a fault $f^{q}$ within the set $\mathcal{R}$ of all ARRs is empty, $f^{q}$ is undetectable. Without loss of generality, it is assumed that all faults in system $\Sigma$ are detectable, i.e., for any fault $f^{q} \in \mathcal{F}$, there exists a subset of ARRs $\mathcal{X} \subseteq \mathcal{R}$ such that $\left|A_{q}^{\mathcal{X}}\right|>0$.

For fault detectability on the set $\mathcal{F}$, the subset $\mathcal{X} \subseteq \mathcal{R}$ is a detecting set if, for every fault $f^{q} \in \mathcal{F},\left|A_{q}^{\mathcal{X}}\right|>0$. Within a detecting set $\mathcal{X}$, two faults $f^{q}, f^{q^{\prime}} \in$ $\mathcal{F}, f^{q} \neq f^{q^{\prime}}$, are distinguishable from each other, if $A_{q}^{\mathcal{X}} \neq A_{q^{\prime}}^{\mathcal{X}}$, i.e., there has a nonempty difference set of $A_{q}^{\mathcal{X}}, A_{q^{\prime}}^{\mathcal{X}}$. Motivated by this, the following definition is given. 
Definition 3.2. Consider a detecting set $\mathcal{X} \subseteq \mathcal{R}$ and two faults $f^{q}, f^{q^{\prime}}, f^{q} \neq f^{q^{\prime}}$. The fault distinguishable $(\mathrm{FD})$ set $D_{q, q^{\prime}}^{\mathcal{X}}$ with respect to $f^{q}$ and $f^{q^{\prime}}$ within $\mathcal{X}$ is the symmetric difference of their corresponding FA sets, i.e., $D_{q, q^{\prime}}^{\mathcal{X}}=D_{q^{\prime}, q}^{\mathcal{X}} \triangleq A_{q}^{\mathcal{X}} \Delta A_{q^{\prime}}^{\mathcal{X}}=$ $\left\{A_{q}^{\mathcal{X}} \cup A_{q^{\prime}}^{\mathcal{X}}\right\} \backslash\left\{A_{q}^{\mathcal{X}} \cap A_{q^{\prime}}^{\mathcal{X}}\right\}$.

Two faults $f^{q}, f^{q^{\prime}}$ are distinguishable within a detecting set $\mathcal{X}$ iff $\left|D_{q, q^{\prime}}^{\mathcal{X}}\right|>0$. On the other hand, if $\left|D_{q, q^{\prime}}^{\mathcal{X}}\right|=0$, a pair $\left(f^{q}, f^{q^{\prime}}\right) \in \mathcal{F} \times \mathcal{F}$ are non-distinguishable from each other. To tackle the case of $\left|D_{q, q^{\prime}}^{\mathcal{X}}\right|=0$, the isolability index $I_{q}^{\mathcal{X}}$ of a fault $f^{q}$ within $\mathcal{X}$ is defined as the number of faults $f^{q^{\prime} \prime} s \in \mathcal{F} \backslash\left\{f^{q}\right\}$ which are distinguishable from fault $f^{q}$, i.e., $I_{q}^{\mathcal{X}} \triangleq\left|\left\{f^{q^{\prime}} \in \mathcal{F} \backslash\left\{f^{q}\right\}:\left|D_{q, q^{\prime}}^{\mathcal{X}}\right|>0\right\}\right|$. Within a detecting set $\mathcal{X} \subseteq \mathcal{R}$, a fault $f^{q}$ is isolable in a few senses as follows

- fully isolable if for all $f^{q^{\prime}} \in \mathcal{F} \backslash\left\{f^{q}\right\},\left|D_{q, q^{\prime}}^{\mathcal{X}}\right|>0$;

- maximumly isolable if at least one fault $f^{q^{\prime}} \in \mathcal{F} \backslash\left\{f^{q}\right\}$ is non-distinguishable from it and its isolability index $I_{q}^{\mathcal{X}}$ is equal to the maximum isolability index $I_{q}^{M}$ defined within the set $\mathcal{R}$ as $I_{q}^{M} \triangleq\left|\left\{f^{q^{\prime}} \in \mathcal{F} \backslash\left\{f^{q}\right\}:\left|D_{q, q^{\prime}}^{\mathcal{R}}\right|>0\right\}\right|$.

Therefore, fault isolability on the set $\mathcal{F}$ can be categorized into

- full isolability if every $f^{q} \in \mathcal{F}$ is fully isolable;

- maximum isolability if one fault in $\mathcal{F}$ is maximumly isolable and the rest of the faults are fully/maximumly isolable.

In a similar way to fault detectability, a new subset of ARRs is introduced for fault isolability on the fault set of $\mathcal{F}$.

Definition 3.3. A detecting set $\mathcal{X} \subseteq \mathcal{R}$ is defined as an isolating set if full/maximum isolability on the fault set $\mathcal{F}$ is achieved.

In addition, let $\mathbb{I}_{\mathcal{X}}$ be the set of all isolating sets. 


\subsection{Problem Formulation}

In this chapter, the sensor placement problem is to place a minimum number of candidate sensors to diagnose all possible faults of concern. Based on Section 3.1, this sensor placement problem can be solved by finding an ARR set $\mathcal{X}^{*} \subseteq \mathcal{R}$ such that each fault in $\mathcal{F}$ is fully/maximumly isolable (i.e., $\mathcal{X}^{*}$ is an isolating set) and that $\mathcal{X}^{*}$ involves the least number of candidate sensors. For a subset of ARRs $\mathcal{X} \subseteq \mathcal{R}$

$$
n_{\text {sen }}(\mathcal{X})=\left|\cup_{r_{n} \in \mathcal{X}} S_{r_{n}}\right|
$$

denotes the number of candidate sensors involved in $\mathcal{X}$. Then, the sensor placement can be formulated as the following optimization problem

$$
\mathcal{X}^{*}=\underset{\mathcal{X} \in \mathbb{I}_{\mathcal{X}}}{\arg \min } n_{\text {sen }}(\mathcal{X})
$$

and the optimal set of candidate sensors for fault detectability and isolability in system $\Sigma$ is $\underset{r_{n} \in \mathcal{X}^{*}}{\cup} S_{r_{n}}$.

In order to reduce the search space of the optimization problem (3.1), a new concept of the minimal isolating set is introduced as follows.

Definition 3.4. An isolating set $\mathcal{X} \subseteq \mathcal{R}$ is called a minimal isolating (MI) set if any proper subset of $\mathcal{X}$ is not an isolating set.

Let $\mathbb{M}_{\mathcal{X}}$ be the set of all MI sets. Obviously, $\mathbb{M}_{\mathcal{X}} \subseteq \mathbb{I}_{\mathcal{X}}$. The following property concerns the relationship between the number of ARRs in any MI set and the number of faults $Q$.

Property 3.1. The number of $A R R$ s in any $M I$ set $\mathcal{X} \in \mathbb{M}_{\mathcal{X}}$ is upper-bounded by the number of faults $Q$, i.e., $|\mathcal{X}| \leq Q$.

Proof. Property 3.1 is proved by induction. Consider $Q=2$ faults. If any MI set 
$\mathcal{X} \in \mathbb{M}_{\mathcal{X}}$ fulfills full isolability on $\mathcal{F}, \mathcal{X}$ has two ARRs. In the other case, if $\mathcal{X}$ fulfills maximum isolability, $\mathcal{X}$ has one ARR. Therefore, $|\mathcal{X}| \leq 2=Q, \mathcal{X} \in \mathbb{M}_{\mathcal{X}}$.

Now assume that if $Q=k$, Property 3.1 is true. It is necessary to prove that Property 3.1 is also true for $Q=k+1$. For $Q=k+1$ faults, the fault set $\mathcal{F}$ can be rewritten as $\mathcal{F}=\left\{f^{1}, f^{2}, \ldots, f^{k}, f^{*}\right\}$. Let $\mathbb{M}_{\mathcal{X}}^{\prime}$ be the set of all MI sets for the first $k$ faults $f^{1}, f^{2}, \cdots, f^{k}$ in $\mathcal{F}$. Then, from the induction hypothesis, $\left|\mathcal{X}_{1}\right| \leq k$ for any $\mathcal{X}_{1} \in \mathbb{M}_{\mathcal{X}}^{\prime}$. For any $\mathrm{MI}$ set $\mathcal{X} \in \mathbb{M}_{\mathcal{X}}$ (for $k+1$ faults in $\left.\mathcal{F}\right)$, because $\mathcal{X}$ is also an isolating set for $k$ faults $f^{1}, f^{2}, \cdots, f^{k}$, it is clear that $\exists \mathcal{X}_{1} \subseteq \mathcal{X}$ such that $\mathcal{X}_{1} \in \mathbb{M}_{\mathcal{X}}^{\prime}$ (i.e., $\mathcal{X}_{1}$ is a MI set for $\left.f^{1}, f^{2}, \cdots, f^{k}\right)$. The proof of this part can be divided into three cases.

- In the first case, fault $f^{*}$ cannot be distinguishable from one fault in $\mathcal{F} \backslash\left\{f^{*}\right\}$ within the set $\mathcal{R}$. $\mathcal{X}_{1}$ fulfills maximum isolability on the fault set $\mathcal{F}$ and thus is also the isolating set for $k+1$ faults. Note that $\mathcal{X}_{1} \subseteq \mathcal{X}$. Therefore, $\mathcal{X}_{1}=\mathcal{X}$ and thus $|\mathcal{X}| \leq k<k+1$.

- In the second case, fault $f^{*}$ is fully isolable within $\mathcal{X}_{1}$. Hence, $\mathcal{X}_{1}$ is the isolating set for $k+1$ faults. Due to $\mathcal{X}_{1} \subseteq \mathcal{X}$ and $\mathcal{X} \in \mathbb{M}_{\mathcal{X}}, \mathcal{X}_{1}=\mathcal{X}$ and thus $|\mathcal{X}| \leq k<k+1$.

- In the last case, fault $f^{*}$ can be fully isolable within $\mathcal{X}$ (not within $\mathcal{X}_{1}$ ) and $\mathcal{X}_{1} \subset \mathcal{X}$. Let $\mathcal{X}_{2}=\mathcal{X} \backslash \mathcal{X}_{1}$; and FA sets $A_{1}^{\mathcal{X}_{1}}, A_{2}^{\mathcal{X}_{1}}, \ldots, A_{k}^{\mathcal{X}_{1}}$ and $A_{*}^{\mathcal{X}_{1}}$ within $\mathcal{X}_{1}$ relate to $f^{1}, f^{2}, \cdots, f^{k}$ and $f^{*}$, respectively. The following introduces the FA set $A_{*}^{\mathcal{X}_{1}}$ from two aspects. First, consider the case where $A_{*}^{\mathcal{X}_{1}}=\varnothing$. To make this fault $f^{*}$ isolable, there exists at least an $\operatorname{ARR} r_{n} \in \mathcal{X}_{2}$ that relates to this fault $f^{*}$. If $\left|\mathcal{X}_{2}\right|>1$, there must exist a proper subset of $\mathcal{X}$ making fault $f^{*}$ fully isolable, which contradicts the fact that $\mathcal{X} \in \mathbb{M}_{\mathcal{X}}$ is the MI set for $k+1$ faults. Consequently, $\left|\mathcal{X}_{2}\right|=1$ when $A_{*}^{\mathcal{X}_{1}}=\varnothing$. Secondly, consider the case where $A_{*}^{\mathcal{X}_{1}} \neq \varnothing$. If $A_{*}^{\mathcal{X}_{1}} \neq A_{i}^{\mathcal{X}_{1}}(i=1,2, \cdots, k)$, then fault $f^{*}$ is fully 
isolable within $\mathcal{X}_{1}$ and $\mathcal{X}_{1}$ becomes the isolating set for $k+1$ faults; but this contradicts the fact that $\mathcal{X}$, rather than $\mathcal{X}_{1}$, is the MI set for $k+1$ faults. Therefore, there is only one fault $f^{k_{*}}\left(1 \leq k_{*} \leq k\right)$ such that $A_{k_{*}}^{\mathcal{X}_{1}}=A_{*}^{\mathcal{X}_{1}}$, if $\mathcal{X}_{1}$ achieves full isolability on the fault set $\left\{f^{1}, f^{2}, \ldots, f^{k}\right\}$. On the other hand, if $\mathcal{X}_{1}$ fulfills maximum isolability on the fault set $\left\{f^{1}, f^{2}, \ldots, f^{k}\right\}$, there exists $h(h \geq 1)$ faults $f^{k_{j}}, 1 \leq j \leq h,\left(1 \leq k_{j} \leq k\right)$ such that $A_{k_{j}}^{\mathcal{X}_{1}}=A_{*}^{\mathcal{X}_{1}}, 1 \leq$ $j \leq h$. Because $\mathcal{X}_{1}$ is a MI set for faults $f^{1}, f^{2}, \ldots, f^{k}$, faults $f^{k_{j}}, 1 \leq j \leq h$, cannot be distinguishable from one another within $\mathcal{X}$. In other words, FA sets $A_{k_{j}}^{\mathcal{X}}, 1 \leq j \leq h$, within $\mathcal{X}$ with respect to faults $f^{k_{j}}, 1 \leq j \leq h$, are same. Therefore, fault $f^{*}$ is fully isolable within $\mathcal{X}$, as long as the FA set $A_{k_{j}}^{\mathcal{X}}$ with respect to one fault $f^{k_{j}}$ is different from the FA set $A_{*}^{\mathcal{X}}$ with respect to fault $f^{*}$. Then, without loss of generality, assume that there exists one fault $f^{k_{*}}$ $\left(1 \leq k_{*} \leq k\right)$ such that $A_{k_{*}}^{\mathcal{X}_{1}}=A_{*}^{\mathcal{X}_{1}}$. If each ARR in $\mathcal{X}_{2}$ that relates to both $f^{k_{*}}$ and $f^{*}$, then $f^{k_{*}}$ and $f^{*}$ are not distinguishable; this is impossible. Thus, for distinguishability of two faults $f^{*}, f^{k_{*}}$, there exists at least an $\operatorname{ARR} r_{n} \in \mathcal{X}_{2}$ that relates to either $f^{*}$ or $f^{k_{*}}$ (but not both). If $\left|\mathcal{X}_{2}\right|>1$, there must exist a proper subset of $\mathcal{X}$ to make fault $f^{*}$ fully isolable, which also contradicts the fact that $\mathcal{X} \in \mathbb{M}_{\mathcal{X}}$ is the MI set for $k+1$ faults. As a result, $\left|\mathcal{X}_{2}\right|=1$ when $A_{*}^{\mathcal{X}_{1}} \neq \varnothing$. Hence, $|\mathcal{X}|=\left|\mathcal{X}_{1}\right|+\left|\mathcal{X}_{2}\right| \leq k+1$.

By summarizing all above, this property follows. The proof is finished.

Any isolating set $\mathcal{X} \in \mathbb{I}_{\mathcal{X}} \backslash \mathbb{M}_{\mathcal{X}}$ has a proper subset $\mathcal{X}^{\prime} \in \mathbb{M}_{\mathcal{X}}$, thereby resulting in $n_{\text {sen }}(\mathcal{X}) \geq n_{\text {sen }}\left(\mathcal{X}^{\prime}\right)$. Then, by substituting the search space $\mathbb{I}_{\mathcal{X}}$ in (3.1) by $\mathbb{M}_{\mathcal{X}}$, the optimization problem is equivalent to

$$
\mathcal{X}^{*}=\underset{\mathcal{X} \in \mathbb{M}_{\mathcal{X}}}{\arg \min } n_{\text {sen }}(\mathcal{X})
$$

In addition, the optimal set of ARRs $\mathcal{X}^{*}$ of (3.2) is closely related to a fault set with 
the fixed number of faults. However, in the system $\Sigma$, the fault set $\mathcal{F}$ consists of $Q_{1}$ system faults that are predefined and $Q_{2}$ sensor faults that are variable as different candidate sensors are chosen. Note that these $Q_{2}$ sensor faults are mostly determined by the isolability result of $Q_{1}$ system faults. Therefore, the sensor placement problem in system $\Sigma$ can be divided into three subproblems: (i) the search of the optimal MI set for $Q_{1}$ system faults, (ii) the determination of $Q_{2}$ candidate sensors for sensor placement and (iii) the search of the optimal set $\mathcal{X}^{*}$ for the set $\mathcal{F}$ with $Q_{1}+Q_{2}$ faults. Obviously, the above mentioned properties, definitions and the formulated optimization problem are true for the set $\mathcal{F}$ with either $Q=Q_{1}$ or $Q=Q_{1}+Q_{2}$ faults. In the following section, a DP approach is developed to deal with subproblems (i) and (iii) for seeking the optimal set of ARRs on a fault set with the fixed number of faults.

\subsection{Dynamic Programming for the Optimal MI Set}

To avoid notational mix-up with the fault set $\mathcal{F}$ in system $\Sigma$, a set $\mathcal{F}^{\prime}$ with $Q^{\prime}$ fixed faults is introduced in this section. Therefore, the number of FD sets is $\frac{Q^{\prime 2}-Q^{\prime}}{2}$. For an isolating set $\mathcal{X} \in \mathbb{I}_{\mathcal{X}}$, the relationship of FD sets with ARRs in $\mathcal{R}$ and that of candidate sensors in $\mathcal{S}$ with ARRs can be visualized graphically in Figure 3.1, where hexagons, ovals and circles represent FD sets, ARRs and candidate sensors, respectively. In this graphical model, an arrow from a FD set $D_{q, q^{\prime}}^{\mathcal{X}}$ to an $\mathrm{ARR}$ $r_{n} \in \mathcal{R}$ indicates $r_{n} \in D_{q, q^{\prime}}^{\mathcal{X}}$; and meanwhile an arrow from a candidate sensor $s_{k} \in \mathcal{S}$ to an ARR $r_{n}$ means $s_{k} \in S_{r_{n}}$. Recall that any FD set $D_{q, q^{\prime}}^{\mathcal{X}} \subseteq \mathcal{X}$ according to Definition 3.2. In the sense of fault diagnosability, a subset $\mathcal{X}$ is said to include a FD set $D_{q, q^{\prime}}^{\mathcal{X}}$ if $D_{q, q^{\prime}}^{\mathcal{X}} \neq \varnothing$, or, equivalently saying, there is at least one arrow from a FD set to an ARR in $\mathcal{X}$ in the graphical model. 


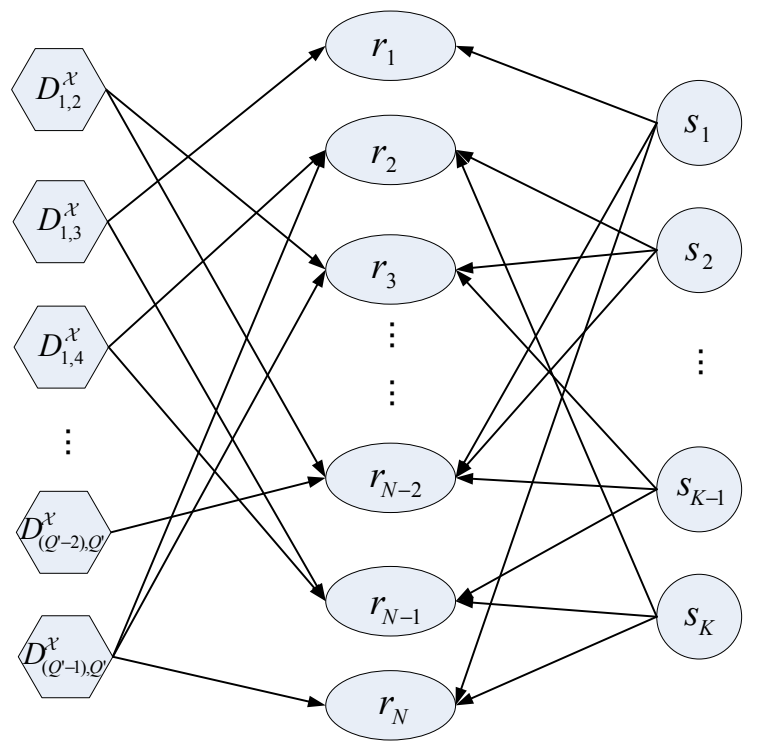

Figure 3.1: A graphical model with respect to an isolating set $\mathcal{X}$.

According to Definition 3.3, an isolating set $\mathcal{X}$ fully/maximally includes all FD sets. In other words, all FD sets fully/maximally point towards the isolating set $\mathcal{X}$ in the graphical model. To easily present DP in search of the isolating set $\mathcal{X}$, let $u$ be a binary vector of size $\frac{Q^{\prime 2}-Q^{\prime}}{2}$ to represent the extent to all FD sets included by $\mathcal{X}$ where 1 means that the corresponding $\mathrm{FD}$ set is included and 0 otherwise. For convenience, $u$ is called the state vector (of all FD sets), let $\mathbf{1} / \mathbf{1}_{m}$ be the state vector denoting all FD sets fully/maximally included. Let $\mathcal{X}_{u}^{i} \subseteq \mathcal{R}$ be a subset of $i$ ARRs that possesses the state vector $u$ and let $\mathbb{X}_{u}^{i}$ be the set of all possible $\mathcal{X}_{u}^{i \prime} s$. Additionally, the symbol $N^{\prime}$ denotes the number of state vectors $u^{\prime} s$ associated with subsets $\mathcal{X}_{u}^{1 \prime} s \subset \mathcal{R}$, i.e., $N^{\prime}=\left|\left\{u: \mathbb{X}_{u}^{1} \neq \varnothing\right\}\right|$. Note that each ARR is in one-to-one correspondence with a subset $\mathcal{X}_{u}^{1}$ of $\mathcal{R}$ and different subsets $\mathcal{X}_{u}^{1 \prime} s$ may possess the same state vector $u$. Hence, $N^{\prime} \leq N$.

\subsubsection{Dynamic Programming}

As a result of Property 3.1, to find the optimal MI set $\mathcal{X}^{*} \in \mathbb{M}_{\mathcal{X}}$ that possesses the state vector $\overrightarrow{\mathbf{1}} / \overrightarrow{\mathbf{1}}_{m}$, one can divide the optimization problem (3.2) into 
$I=\min \left\{N^{\prime}, Q^{\prime}\right\}$ subproblems such that the $i$-th subproblem, $i=1,2, \ldots, I$, only considers subsets $\mathcal{X}^{\prime} s$ of no more than $i$ ARRs. Additionally, note that the optimization problem (3.2) possibly has multiple solutions and assume that the operation "arg $\min n_{\text {sen }}(\mathcal{X}) "$ returns a set of subsets $\mathcal{X}^{\prime}$ s. Thus let $\mathbb{X}^{*}$ denote the set of all $\mathcal{X}^{* \prime} s$. Recall that for a subset $\mathcal{X} \subseteq \mathcal{R}$, the number of candidate sensors related to the subset $\mathcal{X}$ is given by $n_{\text {sen }}(\mathcal{X})$. Then, each $i$-th subproblem, $i=1,2, \ldots, I$, can be formulated as

$$
\mathbb{X}^{*}[i]=\underset{\mathcal{X} \text { possessess } \overrightarrow{\mathbf{1}} / \overrightarrow{\mathbf{1}}_{m} \text { and }|\mathcal{X}| \leq i}{\arg \min } n_{\text {sen }}(\mathcal{X}),
$$

and the set of solutions to (3.2) is

$$
\mathbb{X}^{*}=\mathbb{X}^{*}[I]
$$

From the operations (3.3) and (3.4), all subproblems of the DP for the optimization problem (3.2) can be derived as follows.

Each $i$-th subproblem of the DP, $i=1,2, \ldots, I$, consists of all $2^{\left(Q^{\prime 2}-Q^{\prime}\right) / 2}$ possible state vectors and each state vector $u$ in the $i$-th subproblem is associated with the set $\mathbb{X}^{D P}[i][u]$ where each $\mathcal{X} \in \mathbb{X}^{D P}[i][u]$ contains no more than $i$ ARRs, possesses $u$ and has the least number of related candidate sensors.

More specifically, at the first subproblem (i.e., $i=1$ ), the DP is initialized by

$$
\mathbb{X}^{D P}[1][u]=\left\{\begin{array}{ll}
\underset{\mathcal{X} \in \mathbb{X}_{u}^{1}}{\arg \min } n_{\text {sen }}(\mathcal{X}) & \text { if } \mathbb{X}_{u}^{1} \neq \varnothing \\
\varnothing & \text { if } \mathbb{X}_{u}^{1}=\varnothing
\end{array} .\right.
$$

At each later $i$-th subproblem, $2 \leq i \leq I$, the DP update is

$$
\begin{array}{r}
\mathbb{X}^{D P}[i][u]=\underset{\mathcal{X} \in \mathbb{X}^{D P}[i-1][u] \text { and } \mathcal{X}_{u^{\prime}}^{1} \cup \mathcal{X}_{u^{\prime \prime}}^{i-1} \in \mathbb{X}_{u}^{i}: \mathcal{X}_{u^{\prime}}^{1} \in \mathbb{X}_{u^{\prime}}^{1}, \mathcal{X}_{u^{\prime \prime}}^{i-1} \in \mathbb{X}_{u^{\prime \prime}}^{i-1}}{\arg \min } \\
\left.n_{\text {sen }}(\mathcal{X}), n_{\text {sen }}\left(\mathcal{X}_{u^{\prime}}^{1} \cup \mathcal{X}_{u^{\prime \prime}}^{i-1}\right)\right\},
\end{array}
$$


where the term $\mathcal{X}_{u^{\prime}}^{1} \cup \mathcal{X}_{u^{\prime \prime}}^{i-1} \in \mathbb{X}_{u}^{i}: \mathcal{X}_{u^{\prime}}^{1} \in \mathbb{X}_{u^{\prime}}^{1}, \mathcal{X}_{u^{\prime \prime}}^{i-1} \in \mathbb{X}_{u^{\prime \prime}}^{i-1}$ indicates that the union of $\mathcal{X}_{u^{\prime}}^{1} \in \mathbb{X}_{u^{\prime}}^{1}$ and $\mathcal{X}_{u^{\prime \prime}}^{i-1} \in \mathbb{X}_{u^{\prime \prime}}^{i-1}$ possesses the state vector $u=u^{\prime} \mid u^{\prime \prime}$. In (3.6), it is possible that $\mathbb{X}^{D P}[i][u]=\varnothing$ when $\mathbb{X}^{D P}[i-1][u]=\varnothing$ and $\mathbb{X}_{u}^{i}=\varnothing$.

Finally, the set of solutions to (3.2) is given by

$$
\mathbb{X}^{*}=\mathbb{X}^{D P}[I][\overrightarrow{\mathbf{1}}] \text { or } \mathbb{X}^{D P}[I]\left[\overrightarrow{\mathbf{1}}_{m}\right]
$$

$\mathbb{X}^{D P}[I][\overrightarrow{\mathbf{1}}]\left(\right.$ or $\left.\mathbb{X}^{D P}[I]\left[\overrightarrow{\mathbf{1}}_{m}\right]\right)$ denotes the solution space where each member $\mathcal{X}^{*}$ contains the least number of sensors and possesses the state vector $\overrightarrow{\mathbf{1}}$ (or $\overrightarrow{\mathbf{1}}_{m}$ ).

\subsubsection{Low Complexity Dynamic Programming (LCDP)}

Note that in the DP above, the update (3.6) is very computationally expensive because at each state vector $u$ of the $i$-th subproblem, all possible $\mathcal{X}_{u}^{i-1 \prime} s \in \mathbb{X}_{u}^{i-1}$ $(i>2)$ need to be enumerated previously. In order to lower the computational complexity, the search space $\mathbb{X}_{u^{\prime \prime}}^{i-1}(i>1)$ in (3.6) can be replaced by $\mathbb{X}^{D P}[i-1]\left[u^{\prime \prime}\right]$ $\left(\mathbb{X}^{D P}[i-1]\left[u^{\prime \prime}\right] \subseteq \mathbb{X}_{u^{\prime \prime}}^{i-1}\right)$, which results in the following suboptimal update

$$
\begin{aligned}
& \mathbb{X}^{D P}[i][u]=\underset{\mathcal{X} \in \mathbb{X}^{D P}[i-1][u] \text { and } \mathcal{X}_{u^{\prime}}^{1} \cup \mathcal{X}_{u^{\prime \prime}}^{i-1} \in \mathbb{X}_{u}^{i}: \mathcal{X}_{u^{\prime}}^{1} \in \mathbb{X}_{u^{\prime}}^{1}, \mathcal{X}_{u^{\prime \prime}}^{i-1} \in \mathbb{X}^{D P}[i-1]\left[u^{\prime \prime}\right]}{\arg \min }\{ \\
& \left.n_{\text {sen }}(\mathcal{X}), n_{\text {sen }}\left(\mathcal{X}_{u^{\prime}}^{1} \cup \mathcal{X}_{u^{\prime \prime}}^{i-1}\right)\right\} \text {, }
\end{aligned}
$$

and the final results in $\mathbb{X}^{D P}[I]\left[\overrightarrow{\mathbf{1}} / \overrightarrow{\mathbf{1}}_{m}\right]$ are possibly suboptimal (instead of the optimal solutions $\left.\mathcal{X}^{* \prime} s\right)$.

Although the complexity of (3.8) is low, the performance may degrade because the search space is only limited to $\mathbb{X}^{D P}[i-1]\left[u^{\prime \prime}\right]$ at each $i$-th subproblem. In the subsequent context, a low complexity dynamic programming (LCDP) is proposed to make the balance between performance and complexity. Intuitively, the performance of (3.8) is improved by increasing its search space while controlling the overall 
complexity of the LCDP. Both intentions (i.e., increasing search space and controlling complexity) are achieved by introducing two operators, clipping and mixing. These two operators make a tradeoff between $\mathbb{X}^{D P}[i-1]\left[u^{\prime \prime}\right]$ that only stores subsets of ARRs with the least number of candidate sensors and $\mathbb{X}_{u^{\prime \prime}}^{i-1}$ that keeps all possible subsets of ARRs. To further reduce the complexity, state vectors $u^{\prime} s$ in (3.8) such that each $\mathbb{X}_{u}^{i}=\varnothing$ in the $i$-th subproblem, $i=1, \ldots, I$, are ignored in the implementation of LCDP.

Here, clipping and mixing operators are described in details. Consider a set $\mathbb{Y}=\left\{\mathcal{Y}_{1}, \mathcal{Y}_{2}, \ldots, \mathcal{Y}_{J}\right\}$, where $\mathcal{Y}_{j} \subseteq \mathcal{R}, j=1,2, \ldots, J$, is a subset of ARRs and a non-negative integer $r$. The clipping operator on $\mathbb{Y}$ with respect to $r$ is defined as

$$
C_{r}(\mathbb{Y})=\left\{\begin{array}{ll}
\left\{\mathcal{Y}_{j} \in \mathbb{Y}: n_{\text {sen }}\left(\mathcal{Y}_{j}\right) \leq r\right\} & \text { if } r>n_{\text {sen }}\left(\mathcal{Y}_{\min }\right) \\
\left\{\mathcal{Y}_{\min }\right\} & \text { if } r \leq n_{\text {sen }}\left(\mathcal{Y}_{\min }\right)
\end{array},\right.
$$

where $\mathcal{Y}_{\min }=\underset{\mathcal{Y}_{j} \in \mathbb{Y}}{\arg \min } n_{\text {sen }}\left(\mathcal{Y}_{j}\right)$ is the subset of ARRs in $\mathbb{Y}$ with the least number of related candidate sensors. For two sets $\mathbb{Y}=\left\{\mathcal{Y}_{1}, \mathcal{Y}_{2}, \ldots, \mathcal{Y}_{J}\right\}$ and $\mathbb{Y}^{\prime}=$ $\left\{\mathcal{Y}_{1}^{\prime}, \mathcal{Y}_{2}^{\prime}, \ldots, \mathcal{Y}_{L}^{\prime}\right\}$, the mixing operator on $\mathbb{Y}$ and $\mathbb{Y}^{\prime}$ is defined as

$$
\begin{array}{r}
\operatorname{Mix}\left(\mathbb{Y}, \mathbb{Y}^{\prime}\right)=\left\{\mathcal{Y}_{j} \in \mathbb{Y}, \mathcal{Y}_{l}^{\prime} \in \mathbb{Y}^{\prime}: n_{\text {sen }}\left(\mathcal{Y}_{j}\right) \leq \min \left\{n_{\text {sen }}\left(\mathcal{Y}_{\max }\right), n_{\text {sen }}\left(\mathcal{Y}_{\text {max }}^{\prime}\right)\right\}\right. \\
\left.n_{\text {sen }}\left(\mathcal{Y}_{l}^{\prime}\right) \leq \min \left\{n_{\text {sen }}\left(\mathcal{Y}_{\max }\right), n_{\text {sen }}\left(\mathcal{Y}_{\max }^{\prime}\right)\right\}\right\},
\end{array}
$$

where $\mathcal{Y}_{\max }=\underset{\mathcal{Y}_{j} \in \mathbb{Y}}{\arg \max } n_{\text {sen }}\left(\mathcal{Y}_{j}\right)$ and $\mathcal{Y}_{\max }^{\prime}=\underset{\mathcal{Y}_{l}^{\prime} \in \mathbb{Y}^{\prime}}{\arg \max } n_{\text {sen }}\left(\mathcal{Y}_{l}^{\prime}\right)$ are the subsets of ARRs in $\mathbb{Y}$ and $\mathbb{Y}^{\prime}$, respectively, with the largest number of related candidate sensors.

Similar to the DP in Section 3.3.1, in the $i$-th subproblem, $i=1,2, \ldots, I$, of the LCDP, each state vector $u$ is associated with the set $\mathbb{V}[i][u]$ where each $\mathcal{X} \in \mathbb{V}[i][u]$ contains no more than $i$ ARRs, possesses $u$ and depends on a threshold $r$. The threshold $r$ is a non-negative integer and must be pre-defined (the guidelines on how to determine $r$ will be described next subsection). The LCDP can be described 
as follows.

At the first subproblem, the LCDP can be initialized as

$$
\mathbb{V}[1][u]=C_{r}\left(\mathbb{X}_{u}^{1}\right)
$$

where $\mathbb{X}_{u}^{1}$ is the set of all possible $\mathcal{X}_{u}^{1 \prime} s$ that possess a state vector $u$.

At each later $i$-th subproblem, $2 \leq i \leq I$, let

$$
\mathbb{V}_{u}^{i}=\left\{\mathcal{X}_{u^{\prime}}^{1} \cup \mathcal{X}_{u^{\prime \prime}}^{i-1}: \mathcal{X}_{u^{\prime}}^{1} \in \mathbb{X}_{u^{\prime}}^{1}, \mathcal{X}_{u^{\prime \prime}}^{i-1} \in \mathbb{V}[i-1]\left[u^{\prime \prime}\right] \text { and } \mathcal{X}_{u^{\prime}}^{1} \cup \mathcal{X}_{u^{\prime \prime}}^{i-1} \in \mathbb{X}_{u}^{i}\right\}
$$

be the set of $\mathcal{X}_{u}^{i \prime} s$ that can be determined by the sets $\mathbb{X}_{u^{\prime}}^{1}$ and $\mathbb{V}[i-1]\left[u^{\prime \prime}\right]$. Then, the LCDP update can be written as

$$
\mathbb{V}[i][u]=\operatorname{Mix}\left(\mathbb{V}[i-1][u], C_{r}\left(\mathbb{V}_{u}^{i}\right)\right)
$$

Finally, the set of solutions (determined by the proposed LCDP) to the optimization problem (3.2) is

$$
\mathbb{X}^{L C D P}=\underset{\mathcal{X} \in \mathbb{V}[I]\left[\overrightarrow{\mathbf{1}} / \overrightarrow{\mathbf{1}}_{m}\right]}{\arg \min } n_{\text {sen }}(\mathcal{X})
$$

$\mathbb{V}[I]\left[\overrightarrow{\mathbf{1}} / \overrightarrow{\mathbf{1}}_{m}\right]$ indicates the solution space regarding to the last subproblem in LCDP, where each member $\mathcal{X}$ involves no more than $r$ sensors and possesses the state vector $\overrightarrow{\mathbf{1}}\left(\right.$ or $\left.\overrightarrow{\mathbf{1}}_{m}\right)$.

\subsubsection{Optimal Solutions by LCDP}

For improvement of the performance, the above subsection has introduced two operators clipping and mixing. If the value of $r$ is not large enough, then not all valuable $\mathcal{X}_{u}^{i \prime} s$ are included in $\mathbb{V}[i][u]$. As a result, the replacement of $\mathbb{X}_{u}^{i}$ by $\mathbb{V}[i][u]$ causes (3.14) to return suboptimal solutions. Otherwise, if the set $\mathbb{X}_{u}^{i} \backslash\left\{\mathbb{X}_{u}^{i} \cap \mathbb{V}[i][u]\right\}$ 
is generated under a desirable value $r$ (i.e., this set does not contain any valuable $\mathcal{X}_{u}^{i \prime}$ at all), the LCDP gives the global optima of (3.2). In summary, the optimality of LCDP completely depends on the threshold $r$. For example, if $r \geq K$ (i.e., the threshold does not reject any subset $\mathcal{X}$ in clipping and mixing operators), obviously, $\mathbb{X}_{u}^{i} \subseteq \mathbb{V}[i][u]$ and thus the solutions to (3.2) determined by LCDP are optimal; however, it takes a lot of computational cost since each $\mathbb{V}[i][u](1 \leq i \leq I)$ possessing $u$ includes all possible subsets $\mathcal{X}^{\prime} s$ of no more that $i$ ARRs. To easily discriminate whether the LCDP gives the global optima of (3.2), the following theorem is introduced as a criterion.

Theorem 3.1. Supposed that $W^{*}$ is the cardinality of the optimal set of candidate sensors in the optimization problem (3.2) (i.e., $W^{*}=n_{\text {sen }}\left(\mathcal{X}^{*}\right)$ ), the solutions to (3.2) determined by LCDP are optimal if the threshold $r$ is set to be greater than or equal to $W^{*}$, i.e., $r \geq W^{*}$.

Proof. $r \geq W^{*}$ is a sufficient condition for the LCDP to return optimal solutions. A subset of ARRs $\mathcal{X}$, which is subject to $n_{\text {sen }}(\mathcal{X})>W^{*}$ and possesses a state vector $u$, unites another subset of ARRs $\mathcal{X}^{\prime}$ which possesses the possible compensation state vector $u^{\prime}$ of $u$ such that $u^{\prime} \mid u=\overrightarrow{\mathbf{1}} / \overrightarrow{\mathbf{1}}_{m}$. As a result, the new subset $\mathcal{X} \cup \mathcal{X}^{\prime}$ possesses the state vector $\overrightarrow{\mathbf{1}} / \overrightarrow{\mathbf{1}}_{m}$ and however the number of candidate sensors related to this new subset $\mathcal{X} \cup \mathcal{X}^{\prime}$ is still greater than $W^{*}$, i.e., $n_{\text {sen }}\left(\mathcal{X} \cup \mathcal{X}^{\prime}\right)>W^{*}$, according to the characteristic of the union operator on two sets. Therefore, the global optimum of (3.2) cannot be missing if such a subset of ARRs $\mathcal{X}$ possessing the state vector $u$ in each subproblem is removed. From another point of view, if $r \geq W^{*}$, all subsets $\mathcal{X}^{\prime} s$ that possibly have contributions to seek all global optima are kept in each subproblem. Consequently this guarantees the LCDP to give optimal solutions to $(3.2)$.

According to Theorem 3.1, if $r \geq W^{*}$, then $n_{\text {sen }}(\mathcal{X})=W^{*}\left(\mathcal{X} \in \mathbb{X}^{L C D P}\right)$; if 
$r<W^{*}$, there are usually two problems encountered. One is that the LCDP finds out the global optima of the optimization problem (3.2) but the set of global optima is incomplete. The other is that the LCDP only searches out the local optima of the optimization problem (3.2). Consequently, both cases return solutions $\mathcal{X} \in \mathbb{X}^{L C D P}$, fulfilling $n_{\text {sen }}(\mathcal{X}) \geq W^{*}$. From this observation, $n_{\text {sen }}(\mathcal{X})$ can be used to validate whether the LCDP seeks all global optima or not, instead of $W^{*}$ which is hard to obtain. If $r \geq n_{\text {sen }}(\mathcal{X})\left(\mathcal{X} \in \mathbb{X}^{L C D P}\right)$, it is concluded that all global optima are sought. If $r<n_{\text {sen }}(\mathcal{X})$, the LCDP cannot guarantee all global optima to be found; for the global optimization, the LCDP needs to be invoked again at $r=n_{\text {sen }}(\mathcal{X})$.

Due to the difficulty of obtaining $W^{*}$, one has to set a proper value to the threshold $r$ in order to reduce the computational complexity. Accordingly, the rule of thumb to choose the threshold $r$ for the LCDP is described as follows. Let $G[i]=\{u$ : $\left.\mathbb{X}_{u}^{i} \cap \mathbb{V}[i][u] \neq \varnothing\right\}$ be the set of all state vectors $u^{\prime} s$ in the $i$-th subproblem such that the set $\mathbb{X}_{u}^{i} \cap \mathbb{V}[i][u]$ associated with $u$ is nonempty. The following empirical formula

$$
r=\left\lceil\frac{\sum_{u \in G[1]} \min _{\mathcal{X} \in \mathbb{V}[1][u]} n_{\text {sen }}(\mathcal{X})}{|G[1]|}+0.5\right\rceil,
$$

which results from several simulations, is used to set the threshold $r$ of the LCDP, where $\lceil\cdot\rceil$ is the ceiling function.

The previous discussion can be summarized in an algorithm. Given a set of all ARRs $\mathcal{R}$, a set of candidate sensors $\mathcal{S}$ and a set of faults $\mathcal{F}^{\prime}$, the pseudo-code to determine all optima to the optimization problem (3.2) is presented in Algorithm 3.1.

In Algorithm 3.1, each subset $\mathcal{X}_{u}^{i}$ is established using FSM $C^{\mathcal{X}_{u}^{i}}$ regarding to $\mathcal{F}^{\prime}$, instead of detailed ARRs. Simultaneously, the number of candidate sensors involved in $\mathcal{X}_{u}^{i}$ is computed from SSM B. Accordingly, Algorithm 3.1 actually demands FSM and SSM extracted from $\mathcal{R}, \mathcal{F}^{\prime}, \mathcal{S}$ and all detailed ARRs are unnecessary to be derived in advance. 


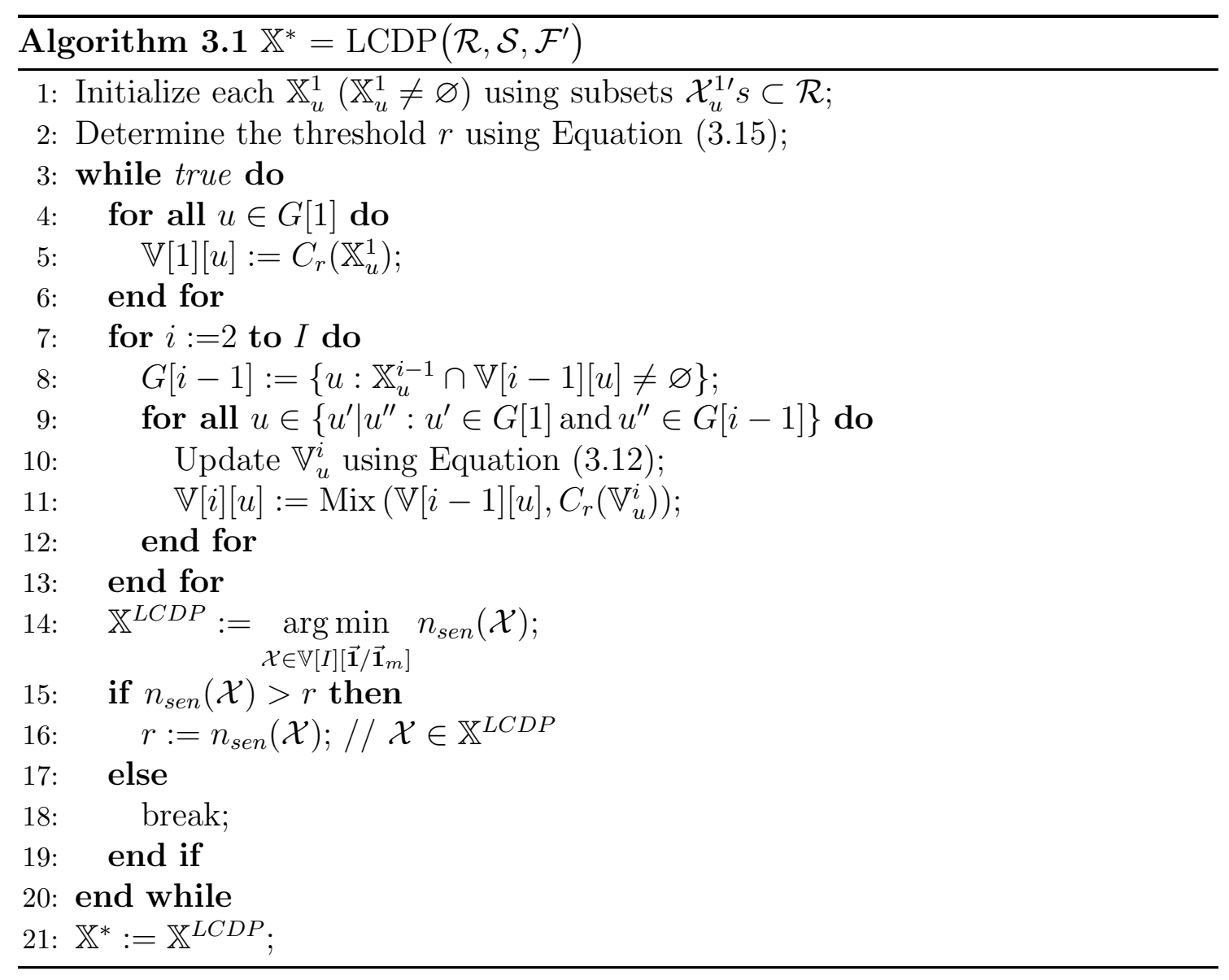

\subsection{Sensor Placement in System $\Sigma$}

The previous section focuses on the determination of the optimal set of ARRs under a fault set with the fixed number of faults using LCDP. In the last section, from the time complexity and space complexity, the performance of LCDP has been optimized. This section provides a solution to determine $Q_{2}$ candidate sensors for sensor placement. An efficient method for sensor placement in system $\Sigma$ is presented in an algorithmic way.

Execution of the LCDP, if $\mathcal{R}, \mathcal{S}$ and $\mathcal{F}_{Q_{1}}$ are given, produces the optimal set of ARRs $\mathcal{X}_{Q_{1}}$. The optimal set of candidate sensors is $\mathcal{S}_{Q_{1}}=\underset{r_{n} \in \mathcal{X}_{Q_{1}}}{\cup} S_{r_{n}}$. In a direct way, sensor faults in $\mathcal{S}_{Q_{1}}$ are considered for isolability and let $\mathcal{F}_{\mathcal{S}_{Q_{1}}}$ denote the set of sensor faults in $\mathcal{S}_{Q_{1}}$. Achieving full/maximum isolability on the fault set $\mathcal{F}=\mathcal{F}_{Q_{1}} \cup \mathcal{F}_{\mathcal{S}_{Q_{1}}}$ may need to utilize additional sensors in $\mathcal{S} \backslash \mathcal{S}_{Q_{1}}$. However, faults of these additional 
sensors in $\mathcal{S} \backslash \mathcal{S}_{Q_{1}}$ must be considered for detectability and/or isolability. Then, the fault set has to be extended and the optimal set of candidate sensors needs to be found again. Generally, there exists such a case that every time the optimal sensor set is searched, new candidate sensors are added and for isolability of their faults, a new searching circle should be done.

In order to avoid the above described situation and efficiently solve the sensor placement problem in system $\Sigma$, it is critical to develop a procedure to determine a set $\mathcal{S}_{Q_{2}}$ of candidate sensors such that $\mathcal{S}_{Q_{1}} \subseteq \mathcal{S}_{Q_{2}}$ and each sensor fault in $\mathcal{S}_{Q_{2}}$ is fully/maximumly isolable by an isolating set $\mathcal{X}$ which achieves full/maximal isolability on the set $\mathcal{F}=\mathcal{F}_{Q_{1}} \cup \mathcal{F}_{Q_{2}}$ and only relates to candidate sensors in $\mathcal{S}_{Q_{2}}$. The following procedure shows how to determine the set $\mathcal{S}_{Q_{2}}$. Given the fault set $\mathcal{F}_{Q_{1}}$, the optimal set of candidate sensors $\mathcal{S}_{Q_{1}}$ and the set $\mathcal{S}$ of all candidate sensors,

i) $\mathcal{S}_{Q_{2}}=\mathcal{S}_{Q_{1}}$;

ii) $\mathcal{F}=\mathcal{F}_{Q_{1}} \cup \mathcal{F}_{Q_{2}}$ and compute the state vector $\mathbf{1} / \mathbf{1}_{m}$ using FSM $C^{\mathcal{R}}$ regarding to $\mathcal{F}$.

iii) extract all ARRs $r_{n} \in \mathcal{R}$ that only relate to candidate sensors in $\mathcal{S}_{Q_{2}}$ and check whether these ARRs possess the state vector $\mathbf{1} / \mathbf{1}_{m}$ or not;

iv) if not, extend $\mathcal{S}_{Q_{2}}$ by adding candidate sensors in $\mathcal{S} \backslash \mathcal{S}_{Q_{1}}$, from 1 to $\left|\mathcal{S} \backslash \mathcal{S}_{Q_{1}}\right|$ (i.e., adding all possible combinations) and then repeat step (ii);

v) if yes, terminate.

Generally, on the fault set $\mathcal{F}_{Q_{1}}$, the LCDP returns several different sets $\mathcal{S}_{Q_{1}}{ }^{\prime} s$. For notational convenience, let $\mathbb{S}_{Q_{1}}$ denote the set of $\mathcal{S}_{Q_{1}}{ }^{\prime}$ s. Clearly, one $\mathcal{S}_{Q_{1}}$ corresponds to one $\mathcal{S}_{Q_{2}}$ and thus a set of $\mathcal{S}_{Q_{2}}{ }^{\prime} s$ can be sought for isolability on the set $\mathcal{F}=$ $\mathcal{F}_{Q_{1}} \cup \mathcal{F}_{Q_{2}}$. Among these $\mathcal{S}_{Q_{2}}{ }^{\prime} s$, ones are chosen that have the least number of 


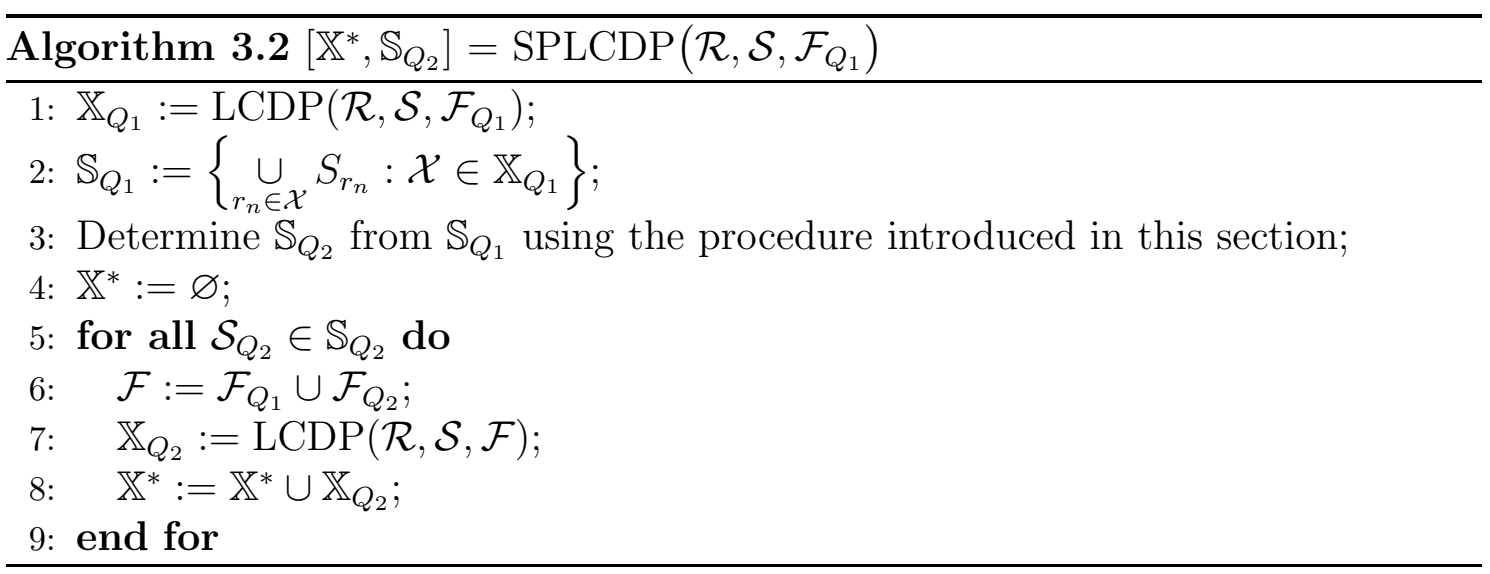

sensors, and let $\mathbb{S}_{Q_{2}}$ represent the set of these chosen $\mathcal{S}_{Q_{2}}{ }^{\prime} s$. Hence, the set $\mathbb{S}_{Q_{2}}$ shows all possible solutions of candidate sensors for sensor placement in system $\Sigma$.

In summary, solving the sensor placement problem of system $\Sigma$ has three steps; and each step rests on its own subroutine. Synthesizing these subroutines can form a complete algorithm for sensor placement. The synthesized algorithm, called sensor placement using LCDP (SPLCDP), is developed to determine the set $\mathbb{X}^{*}$ of all possible $\mathcal{X}^{* \prime} s$ and the set $\mathbb{S}_{Q_{2}}$ of all optimal $\mathcal{S}_{Q_{2}}{ }^{\prime} s$. Given a set of all ARRs $\mathcal{R}$, a set of candidate sensors $\mathcal{S}$ and a set of faults $\mathcal{F}_{Q_{1}}$, the pseudo-code of this algorithm is presented in Algorithm 3.2.

\subsection{Case Studies}

This section illustrates the proposed SPLCDP method using three cases: a twotank system, a CyCab front steering system and a truck diesel engine system. In the latter two systems, the comparison of efficiency is conducted between the SPLCDP and another existing method for sensor placement. In these three examples, sensor faults are thought of as additive faults. For instance, fault $f_{s}^{z}$ representing the fault of sensor $s_{z}$, is modeled by simply adding $f_{s}^{z}$ to the equation which describes the relation between the sensor value $s_{z}$ and the variable $v_{z}$ according to $s_{z}=v_{z}+f_{s}^{z}$. 


\subsubsection{A Two-tank System}

The two-tank system in Figure 3.2 consists of two tanks, a centrifugal pump (with a mass flow $q_{i n}[\mathrm{~kg} / \mathrm{s}]$ ) and one valve represented by $V_{1}$. In this system, the valve $V_{1}$ is assumed to always open. According to the hydraulic principle, the dynamics of this two-tank system are described by the following four equations

$$
\begin{aligned}
& E_{1}: \frac{A_{1}+f^{1}}{g} \dot{p_{1}}=q_{i n}-q_{1} \\
& E_{2}: \frac{A_{2}+f^{2}}{g} \dot{p_{2}}=q_{1}-q_{2} \\
& E_{3}: q_{1}=\left(C d_{1}+f^{3}\right) \sqrt{p_{1}-p_{2}} \\
& E_{4}: q_{2}=C d_{2} \sqrt{p_{2}},
\end{aligned}
$$

where $g\left[\mathrm{~m} / \mathrm{s}^{2}\right]$ is the gravitational acceleration and $q_{1}, q_{2}, p_{1}, p_{2}$ are four unknown variables. More specifically, $q_{1}, q_{2}$ are the mass flows through valve $V_{1}$ and the bottom hole of tank $T_{2}$, respectively; and $p_{1}, p_{2}$ are two pressure readings at the bottom of two tanks, respectively. In equations $E_{1}$ and $E_{2}, A_{1}, A_{2}\left[\mathrm{~m}^{2}\right]$ are crosssection areas of two tanks. $C d_{1}, C d_{2}[\sqrt{\mathrm{kg} \cdot \mathrm{m}}]$ are coefficients of discharge of the valve and the bottom hole in equations $E_{3}, E_{4}$.

In this two-tank system, system faults are modeled as additional values of the corresponding parameters. For instance, faults $f^{1}, f^{2}$ in equations $E_{1}$ and $E_{2}$ are two leakage faults of tanks $T_{1}, T_{2}$ that denote the cross-section change of two tanks.

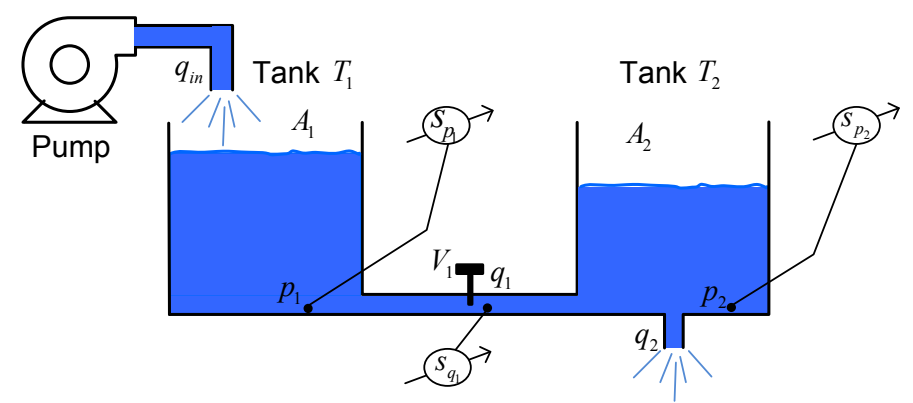

Figure 3.2: A two-tank system with three candidate sensors. 
Table 3.1: The transpose of FSM for the two-tank system

\begin{tabular}{c|ccccccccccc} 
& $r_{1}$ & $r_{2}$ & $r_{3}$ & $r_{4}$ & $r_{5}$ & $r_{6}$ & $r_{7}$ & $r_{8}$ & $r_{9}$ & $r_{10}$ & $r_{11}$ \\
\hline$f^{1}$ & 0 & 0 & 0 & 0 & 1 & 1 & 1 & 1 & 1 & 1 & 1 \\
$f^{2}$ & 0 & 1 & 1 & 1 & 0 & 0 & 0 & 1 & 1 & 1 & 1 \\
$f^{3}$ & 1 & 0 & 1 & 1 & 0 & 1 & 1 & 0 & 1 & 1 & 1
\end{tabular}

Table 3.2: The transpose of SSM for the two-tank system

\begin{tabular}{c|ccccccccccc} 
& $r_{1}$ & $r_{2}$ & $r_{3}$ & $r_{4}$ & $r_{5}$ & $r_{6}$ & $r_{7}$ & $r_{8}$ & $r_{9}$ & $r_{10}$ & $r_{11}$ \\
\hline$s_{p_{1}}$ & 1 & 0 & 1 & 1 & 1 & 0 & 1 & 1 & 0 & 0 & 1 \\
$s_{q_{1}}$ & 1 & 1 & 0 & 1 & 1 & 1 & 0 & 0 & 0 & 1 & 0 \\
$s_{p_{2}}$ & 1 & 1 & 1 & 0 & 0 & 1 & 1 & 1 & 1 & 0 & 0
\end{tabular}

Additionally, fault $f^{3}$ represents the blockage of valve $V_{1}$. If valve $V_{1}$ is clogged, then $f^{3} \neq 0$. Consequently, the set of system faults in this system is $\mathcal{F}_{Q_{1}}=\left\{f^{1}, f^{2}, f^{3}\right\}$. This two-tank system concerns three candidate sensors $s_{p_{1}}, s_{q_{1}}, s_{p_{2}}$ measuring unknown variables $p_{1}, q_{1}, p_{2}$. The corresponding sensor equations are listed as follows

$$
\begin{aligned}
& E_{5}: s_{p_{1}}=p_{1}+f_{s}^{p_{1}}, \\
& E_{6}: s_{q_{1}}=q_{1}+f_{s}^{q_{1}}, \\
& E_{7}: s_{p_{2}}=p_{2}+f_{s}^{p_{2}} .
\end{aligned}
$$

From the above seven equations, a total of eleven ARRs can be found. Thus, FSM and SSM of this two-tank system are given in Table 3.1 and 3.2.

Consider the first use of the LCDP (i.e., the first step of the SPLCDP). From Table 3.1, one can find that the LCDP has $I=\min \{4,3\}=3$ subproblems and the set of all state vectors in the first subproblem is $G[1]=\{[110],[101],[011],[000]\}$. Using the empirical equation (3.15), the threshold is set as $r=3$. As a result of the LCDP, only one optimal set of candidate sensors is $\mathcal{S}_{Q_{1}}=\left\{s_{p_{1}}, s_{p_{2}}\right\}$. Then, the procedure described in Section 3.4 is invoked, passing parameters $\mathcal{F}_{Q_{1}}=\left\{f^{1}, f^{2}, f^{3}\right\}, \mathcal{S}_{Q_{1}}=$ $\left\{s_{p_{1}}, s_{p_{2}}\right\}$ and $\mathcal{S}=\left\{s_{p_{1}}, s_{q_{1}}, s_{p_{2}}\right\}$ and thus only one optimal set of candidate sensors $\mathcal{S}_{Q_{2}}=\left\{s_{p_{1}}, s_{p_{2}}\right\}$ is sought. The third step of the SPLCDP is to implement the LCDP again with the fault set $\mathcal{F}=\left\{f^{1}, f^{2}, f^{3}, f_{s}^{p_{1}}, f_{s}^{p_{2}}\right\}$. Consequently, the resulting opti- 
Table 3.3: The FSM for an optimal set $\mathcal{X}^{*}$ of ARRs in the two-tank system

\begin{tabular}{c|ccccc} 
& $f^{1}$ & $f^{2}$ & $f^{3}$ & $f_{s}^{p_{1}}$ & $f_{s}^{p_{2}}$ \\
\hline$r_{3}$ & 0 & 1 & 1 & 1 & 1 \\
$r_{8}$ & 1 & 1 & 0 & 1 & 1 \\
$r_{9}$ & 1 & 1 & 1 & 0 & 1 \\
$r_{11}$ & 1 & 1 & 1 & 1 & 0
\end{tabular}

mal set $\mathbb{X}^{*}$ contains five optimal sets $\mathcal{X}^{* \prime} s$. Each set $\mathcal{X}^{*}$ consists of four ARRs. For instance, an optimal set of ARRs $\mathcal{X}^{*}=\left\{r_{3}, r_{8}, r_{9}, r_{11}\right\}$ achieves full isolability on the fault set $\mathcal{F}=\left\{f^{1}, f^{2}, f^{3}, f_{s}^{p_{1}}, f_{s}^{p_{2}}\right\}$, which can be seen from the corresponding FSM shown in Table 3.3.

\subsubsection{A CyCab Front Steering System}

The electro-hydraulic steering system of a CyCab mobile robot has been studied in [57] for fault detectability and isolability. The CyCab is an electric passenger transporter with four driving wheels and a dual steering system; it carries some sensor locations. Each steering system of the CyCab is identical and independent. Therefore, this chapter employs the CyCab front electro-hydraulic steering system to illustrate the application of the sensor placement approach for fault detectability and isolability.

The CyCab front steering system is depicted in Figure 3.3; it consists of a DC motor, a gear box, a motion controller, a belt, an oil pump, a hydraulic piston, oil tubes, a steering mechanism, wheels and tires. The model of this front steering system is described in Appendix, which is composed of five parts.

In the DC-motor and pump part (see equations A.1-A.5), parameter $u_{i n}$ is the control signal of the steering system; and $k_{1}, k_{2}$ are the voltage-to-current ratio and the current-to-torque ratio, respectively. Besides $k_{1}$ and $k_{2}$, the gear ratio is $k_{3}$ and the belt ratio is $k_{4}$. Variables $\tau_{O}, \tau_{P_{i}}$ and $\tau_{P_{o}}$ represent the torques of DC-motor output, pump input and pump output which are unknown and unmeasurable. The 


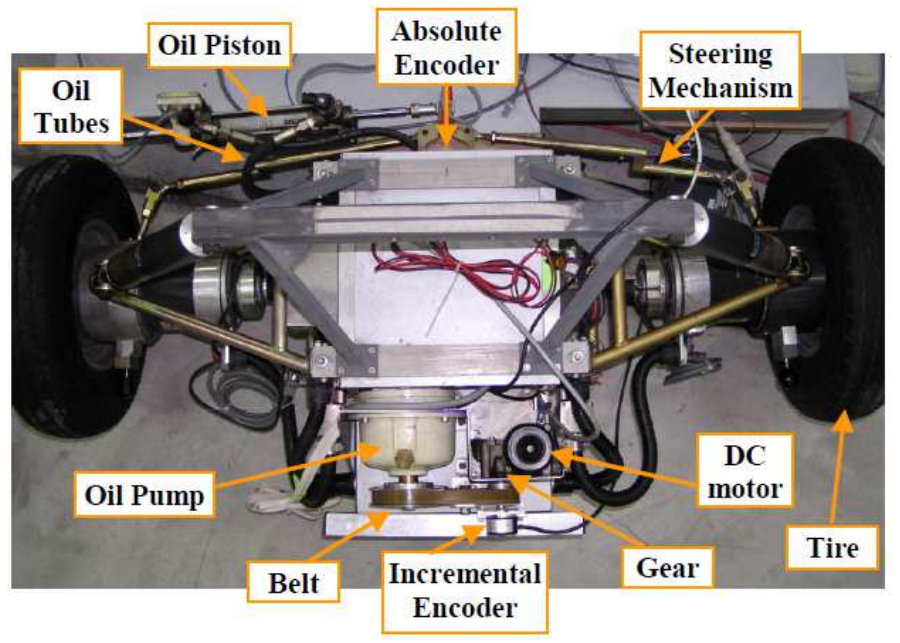

Figure 3.3: The CyCab front steering system.

Table 3.4: Candidate sensors in the CyCab front steering system

\begin{tabular}{ll}
\hline Sensors & Description \\
\hline$s_{\theta_{1}}$ & incremental encoder measuring the position of the gear box \\
$s_{\theta_{2}}$ & absolute encoder measuring the position of the steering mechanism \\
$s_{p_{1,2}}$ & sensor measuring the difference pressure of two tubes \\
$s_{\omega_{1}}$ & sensor of angular speed of DC-motor \\
$s_{\omega_{3}}$ & sensor of angular speed of the steering mechanism \\
$s_{q_{1}}$ & sensor of volume outflow of oil pump \\
\hline
\end{tabular}

other variables (of equations A.1-A.5) $\omega_{1}$ and $\theta_{1}$ are measurable and their corresponding sensors are given in Table 3.4. Following the symbols used in [57], $R_{2 v}$ and $R_{2 c}$ denote the viscous friction and the coulomb friction in the DC-motor; and $J_{1}$ is the DC-motor inertia. The mechanical friction $\left\{R_{3 v}, R_{3 c}\right\}$ and inertia $J_{2}$ model the pump rotor. The DC-motor and pump friction torques are described by $g_{2}(\cdot)$ and $g_{3}(\cdot)$, respectively.

In the hydraulic cylinder part (see equations A.6-A.9), parameter $k_{5}$ is the torqueto-pressure ratio and parameter $A$ is the cross-section area of the piston. In this part, $q_{1}$ is a measurable variable that represents the volume outflow of the oil pump. Variables $p_{1}$ and $p_{2}$ are pressures of two tubes. Variables $F_{\text {pist }}$ and $v_{\text {pist }}$ are the force and velocity acting on the piston. Without the piston internal leakage, parameter $C_{d}$ is identically zero. However, this case study considers the fault of the piston internal 
Chapter 3. Sensor Placement for Fault Isolability Using Low Complexity Dynamic Programming

Table 3.5: Nominal physical parameters

\begin{tabular}{cll||cll}
\hline Parameter & Value & Unit & Parameter & Value & Unit \\
\hline$R_{2 v}$ & $1.017 \mathrm{E}-5$ & $\mathrm{~N} \cdot \mathrm{m} \cdot \mathrm{s}$ & $J_{3}$ & $9.78 \mathrm{E}-2$ & $\mathrm{~kg} \cdot \mathrm{m}^{2}$ \\
$R_{2 c}$ & $6.20 \mathrm{E}-2$ & $\mathrm{~N} \cdot \mathrm{m}$ & $k_{1}$ & 3 & $\mathrm{~A} \cdot \mathrm{V}^{-1}$ \\
$R_{3 v}$ & $3.161 \mathrm{E}-1$ & $\mathrm{~N} \cdot \mathrm{m} \cdot \mathrm{s}$ & $k_{2}$ & 0.0663 & $\mathrm{~N} \cdot \mathrm{m} \cdot \mathrm{A}^{-1}$ \\
$R_{3 c}$ & $3.55 \mathrm{E}-2$ & $\mathrm{~N} \cdot \mathrm{m}$ & $k_{3}$ & $4 / 70$ & \\
$R_{7 v}$ & 33.28 & $\mathrm{~N} \cdot \mathrm{m} \cdot \mathrm{s}$ & $k_{4}$ & $1 / 2$ & \\
$R_{7 c}$ & 22.11 & $\mathrm{~N} \cdot \mathrm{m}$ & $k_{5}$ & $1.024 \mathrm{E}-4$ & $\mathrm{~m}^{3}$ \\
$J_{1}$ & $3.727 \mathrm{E}-5$ & $\mathrm{~kg} \cdot \mathrm{m}^{2}$ & $\mathrm{~A}$ & $4.46 \mathrm{E}-4$ & $\mathrm{~m}^{2}$ \\
$J_{2}$ & $5.627 \mathrm{E}-4$ & $\mathrm{~kg} \cdot \mathrm{m}^{2}$ & & & \\
\hline
\end{tabular}

leakage $f^{\text {pist }}$ that is presented in equation A.8. The Ackerman's steering mechanism (see equations A.10-A.14) can be regarded as a transformer with two sub-moduli $F_{1}\left(\theta_{2}\right)$ and $F_{2}\left(\theta_{2}\right)$ (two nonlinear functions about $\left.\theta_{2}\right)$ [57]. Variables $\tau_{W 1}\left(\omega_{3}\right)$ and $\tau_{W 2}\left(\omega_{4}\right)$ are two torques (angular speeds) through transformations by $F_{1}\left(\theta_{2}\right)$ and $F_{2}\left(\theta_{2}\right) . \omega_{3}$ and $\theta_{2}$ are two measurable variables and thus sensors measure them shown in Table 3.4. Equation A.15 describes the kinematics of wheels. Parameters $\left\{R_{7 v}, R_{7 c}\right\}$ as described in [57] represent the friction between the wheel and the road. Then, the flat-tire fault of the wheel $f^{\text {tire }}$ is introduced to model changes of nominal values of $\left\{R_{7 v}, R_{7 c}\right\} \cdot g_{7}(\cdot)$ describes the friction $\left\{R_{7 v}, R_{7 c}\right\}$ torque loss. The nominal physical parameters of the CyCab steering system are given in Table 3.5.

The last part of this model concerns sensors that are possibly installed in the CyCab front steering system. There are six equations (A.16-A.21) associated with sensors in Table 3.4. All possible faults are listed in Table 3.6. Faults $f^{\text {pist }}, f^{\text {tire }}$ are two system faults and the rest are sensor faults.

From the description above, this front steering system has a set of six candidate sensors, i.e., $\mathcal{S}=\left\{s_{\theta_{1}}, s_{\theta_{2}}, s_{p_{1,2}}, s_{\omega_{1}}, s_{\omega_{3}}, s_{q_{1}}\right\}$, and a set of two system faults, i.e., $\mathcal{F}_{Q_{1}}=\left\{f^{\text {pist }}, f^{\text {tire }}\right\}$. From equations A.1-A.21, a total of 123 MSO sets can be found using the method depicted in [82]. Each MSO set potentially forms an ARR. Accordingly, the set $\mathcal{R}$ of this case study has 123 members. The threshold $r$ is set as 3 using the empirical equation (3.15). The LCDP algorithm is executed to search 
Table 3.6: Faults in the CyCab front steering system

\begin{tabular}{ll}
\hline Faults & \multicolumn{1}{c}{ Description } \\
\hline$f^{\text {pist }}$ & the oil piston internal leakage \\
$f^{\text {tire }}$ & the flat-tire fault of the wheel \\
$f_{s}^{\theta_{1}}$ & the sensor fault of incremental encoder $s_{\theta_{1}}$ \\
$f_{s}^{\theta_{2}}$ & the sensor fault of absolute encoder $s_{\theta_{2}}$ \\
$f_{s}^{p_{1,2}}$ & the fault of difference pressure sensor $s_{p_{1,2}}$ \\
$f_{s}^{\omega_{1}}$ & the fault of angular speed sensor $s_{\omega_{1}}$ \\
$f_{s}^{\omega_{3}}$ & the fault of angular speed sensor $s_{\omega_{3}}$ \\
$f_{s}^{q_{1}}$ & the fault of volumetric flow sensor $s_{q_{1}}$ \\
\hline
\end{tabular}

Table 3.7: Eight optimal sensor sets $\mathcal{S}_{Q_{2}}{ }^{\prime} s \in \mathbb{S}_{Q_{2}}$ for the front steering system

\begin{tabular}{c|cccccc}
$\mathcal{S}_{Q_{2}}$ & $s_{\theta_{1}}$ & $s_{\theta_{2}}$ & $s_{p_{1,2}}$ & $s_{\omega_{1}}$ & $s_{\omega_{3}}$ & $s_{q_{1}}$ \\
\hline 1 & & & & & $\mathrm{X}$ & $\mathrm{X}$ \\
2 & & $\mathrm{X}$ & & $\mathrm{X}$ & & \\
3 & & & & $\mathrm{X}$ & $\mathrm{X}$ & \\
4 & $\mathrm{X}$ & & & & $\mathrm{X}$ & \\
5 & $\mathrm{X}$ & $\mathrm{X}$ & & & & \\
6 & & & $\mathrm{X}$ & & $\mathrm{X}$ & \\
7 & & $\mathrm{X}$ & & & & $\mathrm{X}$ \\
8 & & $\mathrm{X}$ & $\mathrm{X}$ & & &
\end{tabular}

out optimal sets $\mathcal{S}_{Q_{1}}{ }^{\prime} s$ for isolability of faults in $\mathcal{F}_{Q_{1}}$. As a result, the resultant set $\mathbb{S}_{Q_{1}}$ consists of eight optimal sets $\mathcal{S}_{Q_{1}}{ }^{\prime} s$ and simultaneously the resultant set $\mathbb{X}^{*}$ has 76 ARR solutions. Each solution contains two ARRs. Then, the procedure of determining optimal sensor set(s) for isolability of faults in $\mathcal{F}_{Q_{1}} \cup \mathcal{F}_{Q_{2}}$ is invoked and thus the resultant set $\mathbb{S}_{Q_{2}}$ has eight optimal sets which are same as those in $\mathbb{S}_{Q_{1}}$. These eight optimal sets $\mathcal{S}_{Q_{2}}{ }^{\prime} s$ are given in Table 3.7 , where the symbol ' $X$ ' in a row indicates that the corresponding candidate sensor appears in an optimal set $\mathcal{S}_{Q_{2}}$. The last step of the SPLCDP is to re-invoke the LCDP with the different fault set $\mathcal{F}=\mathcal{F}_{Q_{1}} \cup \mathcal{F}_{Q_{2}}$. As a consequence, every optimal set $\mathcal{S}_{Q_{2}}$ in $\mathbb{S}_{Q_{2}}$ is associated with four optimal MI sets and thus the set $\mathbb{X}^{*}$ contains 32 optimal ARR solutions. Every optimal MI set consists of three ARRs. For example, an optimal MI set $\mathcal{X}^{*}=\left\{r_{107}, r_{111}, r_{120}\right\}$ is related to the optimal set $\mathcal{S}_{Q_{2}}=\left\{s_{\theta_{1}}, s_{\theta_{2}}\right\}$ and fulfills full isolability on the fault set $\mathcal{F}$, which can be verified in the corresponding FSM shown 
Table 3.8: The FSM for an optimal MI set $\mathcal{X}^{*}$ in the CyCab front steering system

\begin{tabular}{l|cccc} 
ARR & $f^{\text {pist }}$ & $f^{\text {tire }}$ & $f_{s}^{\theta_{1}}$ & $f_{s}^{\theta_{2}}$ \\
\hline 107 & 1 & 1 & 0 & 1 \\
111 & 0 & 1 & 1 & 1 \\
120 & 1 & 0 & 1 & 1
\end{tabular}

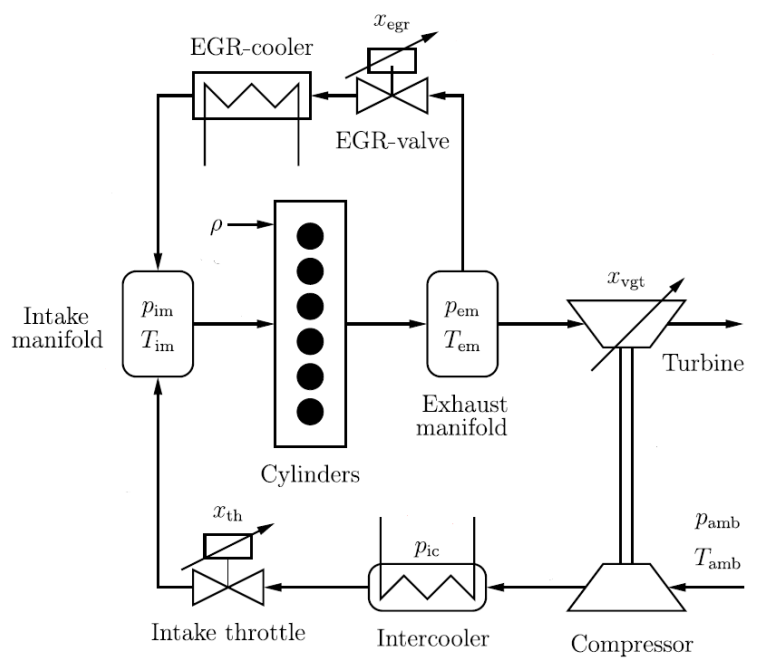

Figure 3.4: Schematic of the truck diesel engine system.

in Table 3.8.

\subsubsection{A Truck Diesel Engine System}

The truck diesel engine system is equipped with exhaust gas recirculation (EGR), EGR-cooler, variable geometry turbochargers (VGT), intake throttle and intercooler, whose schematic is shown in Figure 3.4. The model of the truck diesel engine is described in [115]; for much more details about it, refer to [116]. This model contains 39 equations to describe the dynamic behavior of the engine (see the appendix in [115]). Among the 39 equations, five are differential equations and the remaining equations are algebraic.

Moreover, 43 unknown variables are included in the truck diesel engine model as well as four known variables that represent four actuators. Assume that each actuator has a fault. The detailed description of these four system faults are given 
Table 3.9: Four system faults of the truck diesel engine system

\begin{tabular}{ll}
\hline Fault & Description \\
\hline$f^{x_{t h}}$ & Throttle position actuator fault \\
$f^{x_{e g r}}$ & EGR-valve position actuator fault \\
$f^{x_{v g t}}$ & VGT-valve position actuator fault \\
$f^{\rho}$ & Injected fuel actuator fault \\
\hline
\end{tabular}

Table 3.10: Eight candidate sensors in the truck diesel engine system

\begin{tabular}{ll}
\hline Sensor & Description \\
\hline$s_{p_{a m b}}$ & Ambient pressure sensor \\
$s_{T_{a m b}}$ & Ambient temperature sensor \\
$s_{p_{i c}}$ & Inter-cooler pressure sensor \\
$s_{p_{i m}}$ & Inlet manifold pressure sensor \\
$s_{T_{i m}}$ & Inlet manifold temperature sensor \\
$s_{p_{e m}}$ & Exhaust manifold pressure sensor \\
$s_{T_{e m}}$ & Exhaust manifold temperature sensor \\
$s_{n_{e}}$ & Engine speed sensor \\
\hline
\end{tabular}

in Table 3.9. In addition to system faults, candidate sensors used in this diesel engine model should be defined. Thus, assume that eight unknown variables could be measured by sensors. In other words, there are eight candidate sensors of concern shown in Table 3.10. On these sensor equations and 39 system equations of the diesel engine model, 1640 MSO sets [82] in total can be generated. Then, FSM and SSM of this engine directly arise from these MSO sets. They are passed to the SPLCDP algorithm for sensor placement.

As a result of the SPLCDP, the number of the optimal MI sets for the fault set $\mathcal{F}_{Q_{1}}$ is 108 , every optimal MI set containing 3 ARRs. Simultaneously, 27 optimal

Table 3.11: Five optimal sensor sets $\mathcal{S}_{Q_{2}}{ }^{\prime} s \in \mathbb{S}_{Q_{2}}$ for the truck diesel engine system

\begin{tabular}{|c|c|c|c|c|c|c|c|c|}
\hline$\tilde{\omega}^{\Im}$ & $\omega^{\overparen{ह}}$ & हू. & $\omega^{\circ}$ & $\omega^{ह}$ & है & $\omega^{\tilde{E}}$ & 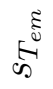 & के \\
\hline 1 & & $\mathrm{X}$ & $\mathrm{X}$ & $\mathrm{X}$ & & $\mathrm{X}$ & $\mathrm{X}$ & $\mathrm{X}$ \\
\hline 2 & $\mathrm{X}$ & & $\mathrm{X}$ & $\mathrm{X}$ & $\mathrm{X}$ & $\mathrm{X}$ & $\mathrm{X}$ & \\
\hline 3 & $\mathrm{X}$ & $X$ & & $\mathrm{X}$ & $\mathrm{X}$ & & $\mathrm{X}$ & X \\
\hline 4 & $X$ & $\mathrm{X}$ & $\mathrm{X}$ & $\mathrm{X}$ & & $X$ & & $\mathrm{X}$ \\
\hline 5 & & $\mathrm{X}$ & $\mathrm{X}$ & & X & $\mathrm{X}$ & X & X \\
\hline
\end{tabular}


Table 3.12: The FSM for an optimal MI set $\mathcal{X}^{*}$ in the truck diesel engine system

\begin{tabular}{|c|c|c|c|c|c|c|c|c|c|c|}
\hline $\mathrm{ARR}$ & $\stackrel{8}{*}$ & & $t$ & $2^{2}$ & 7 & 40 & $\underbrace{2}_{0}$ & 50 & $\underbrace{2} \infty$ & $\sum 0$ \\
\hline 970 & 0 & 1 & 1 & 1 & 1 & 1 & 1 & 1 & 1 & 1 \\
\hline 1204 & 1 & 1 & 1 & 0 & 1 & 1 & 1 & 1 & 1 & 1 \\
\hline 1439 & 1 & 0 & 1 & 1 & 1 & 1 & 1 & 1 & 1 & 1 \\
\hline 1565 & 1 & 1 & 1 & 1 & 0 & 1 & 1 & 1 & 1 & 1 \\
\hline 1601 & 1 & 1 & 0 & 1 & 1 & 1 & 1 & 1 & 1 & 1 \\
\hline 1626 & 1 & 1 & 1 & 1 & 1 & 1 & 0 & 1 & 1 & 1 \\
\hline 1632 & 1 & 1 & 1 & 1 & 1 & 1 & 1 & 0 & 1 & 1 \\
\hline 1637 & 1 & 1 & 1 & 1 & 1 & 1 & 1 & 1 & 0 & 1 \\
\hline 1639 & 1 & 1 & 1 & 1 & 1 & 1 & 1 & 1 & 1 & 0 \\
\hline
\end{tabular}

sets $\mathcal{S}_{Q_{2}}{ }^{\prime} s \in \mathbb{S}_{Q_{2}}$ of candidate sensors for sensor placement are sought. Due to the limitation of length, five optimal sensor sets $\mathcal{S}_{Q_{2}}{ }^{\prime} s \in \mathbb{S}_{Q_{2}}$ are arbitrarily chosen. Table 3.11 shows these five optimal sets $\mathcal{S}_{Q_{2}}$ 's where a ' $X$ ' in the row indicates that a set $\mathcal{S}_{Q_{2}}$ contains the corresponding candidate sensor. Meanwhile, the resulting set $\mathbb{X}^{*}$ contains 27 types of ARR solutions for the fault set $\mathcal{F}=\mathcal{F}_{Q_{1}} \cup \mathcal{F}_{Q_{2}}$, each type resting on an optimal set $\mathcal{S}_{Q_{2}} \in \mathbb{S}_{Q_{2}}$ and having 10 solutions $\mathcal{X}^{* \prime} s \in \mathbb{X}^{*}$. In other words, the set $\mathbb{X}^{*}$ consists of 270 ARR solutions, each solution containing 9 ARRs. One example of 9-ARR solution is taken, which relates to the optimal sensor set $\mathcal{S}_{Q_{2}}=\left\{s_{p_{a m b}}, s_{T_{a m b}}, s_{p_{i c}}, s_{p_{i m}}, s_{p_{e m}}, s_{n_{e}}\right\}$ and whose FSM is given in Table 3.12.

\subsubsection{The Comparison Between the SPLCDP and the BILP}

The binary integer linear programming (BILP) [80] has been used for sensor placement. In the subsequent context, the comparison of the efficiency between the SPLCDP and the BILP is made in the CyCab front steering system and the truck diesel engine system. The BILP is required that each candidate sensor and each ARR are assigned into their desirable cost. Then, following the rule given in the BILP, each sensor is assigned into 1 and each ARR is assigned into 1E-3. Due mostly to the large size of the data, the BILP is implemented by CPLEX12.1 of ILOG 
Table 3.13: The comparison between the SPLCDP and the BILP

(a) CyCab front steering system

\begin{tabular}{llll}
\hline Method & Optimal MI set $\mathcal{X}^{*}(\#)$ & $\mathcal{S}_{Q_{2}}\left(\in \mathbb{S}_{Q_{2}}\right)(\#)$ & Time (sec) \\
\hline BILP $^{\mathrm{a}}$ & 1 & 1 & 0.21 \\
SPLCDP & 32 & 8 & 0.10 \\
\hline
\end{tabular}

(b) Truck diesel engine system

\begin{tabular}{llll}
\hline Method & Optimal MI set $\mathcal{X}^{*}(\#)$ & $\mathcal{S}_{Q_{2}}\left(\in \mathbb{S}_{Q_{2}}\right)(\#)$ & Time (sec) \\
\hline BILP $^{\mathrm{a}}$ & 1 & 1 & 9.19 \\
SPLCDP & 270 & 27 & 0.51 \\
\hline
\end{tabular}

a Set tolerances for the ILOG mathematical programming model solver: Absolute MIP gap tolerance $=1 \mathrm{E}-4$, Integrality tolerance $=9 \mathrm{E}-4$, Relative MIP gap tolerance $=9 \mathrm{E}-4$, Optimality tolerance $=1 \mathrm{E}-4$, Feasibility tolerance $=1 \mathrm{E}-4$.

[117] which is a library of APIs coded by $\mathrm{C}++$ and developed for efficiently solving linear programming problems. For comparison conducted on the same platform, the SPLCDP is also coded by $\mathrm{C}++$. Both algorithms are executed on a personal computer $(\mathrm{OS}=$ windows 7, Memory $=2.96 \mathrm{G}, \mathrm{CPU}=2.8 \mathrm{GHz})$.

In the CyCab front steering system, the BILP is executed 20 times with different initial guesses and the average executed time is $0.21 \mathrm{sec}$ (which is shown in Table $3.13(\mathrm{a}))$. The BILP converges into $\mathcal{X}^{*}=\left\{r_{90}, r_{103}, r_{104}\right\}$ and then the computed sensor solution is $\mathcal{S}_{Q_{2}}=\left\{s_{\omega_{3}}, s_{q_{1}}\right\}$. However, the SPLCDP performs faster than the BILP. The SPLCDP consumes $0.10 \mathrm{sec}$ to give eight optimal sensor sets as well as 32 optimal MI sets. The detailed comparison of these two methods is shown in Table 3.13(a).

In the truck diesel engine system, the average time of 20 BILP executions is 9.19 sec. Every execution of the BILP produces one optimal MI set $\mathcal{X}^{*}=\left\{r_{970}, r_{1204},-\right.$ $\left.r_{1439}, r_{1565}, r_{1601}, r_{1626}, r_{1632}, r_{1637}, r_{1639}\right\}$ and one optimal sensor set $\mathcal{S}_{Q_{2}}=\left\{s_{p_{a m b},}\right.$, $\left.s_{T_{a m b}}, s_{p_{i c}}, s_{p_{i m}}, s_{p_{e m}}, s_{n_{e}}\right\}$. The SPLCDP gives all possible solutions in relation to 27 different optimal sensor sets $\mathcal{S}_{Q_{2}}{ }^{\prime} s$. Table 3.13(b) shows that the executed time of the BILP is about eighteen times longer than that of the SPLCDP. 
The above two case studies have different data sizes. The latter one is larger than the former one. In the CyCab front steering system (with smaller data), the difference between the efficiencies of the BILP and the SPLCDP is not significant. The execution time ratio of the BILP over the SPLCDP is about two; whilst in the truck diesel engine system, this ratio rapidly increases up to eighteen. Therefore, it may be concluded that the SPLCDP performs better on the data with larger size.

\subsection{Summary}

This chapter describes a strategy for solving the sensor placement problem in a system $\Sigma$ by making system faults and sensor faults in $\mathcal{F}$ fully/maximumly isolable. The proposed approach for sensor placement (the SPLCDP) is separated into three parts. The first and third parts are to seek the optimal MI set on fault sets $\mathcal{F}_{Q_{1}}$ and $\mathcal{F}$, respectively. On a set with the fixed number of faults (like $\mathcal{F}_{Q_{1}}$ ), full/maximum isolability as the constraint of sensor placement problem, is fulfilled by finding a MI set which is a new concept to bridge the relationship between the constraint and candidate sensors. To find the optimal MI set, this chapter has visualized graphically the relationships among FD sets, ARRs and candidate sensors; and then has developed the DP algorithm operating on this graphical model. It is noted that the number of state vectors in the DP increases exponentially with the square of the number of faults. For the sake of lowering the computational and space cost, the LCDP algorithm has been proposed to search the optimal MI set $\mathcal{X}^{*}$. In the second part of this approach, a procedure is developed in Section 3.4 based on the set $\mathcal{S}_{Q_{1}}$ to determine the optimal set of candidate sensors. In the CyCab front steering system and the truck diesel engine system, the SPLCDP outperforms the BILP in terms of the efficiency as well as the found optimal solutions, i.e., $\mathcal{X}^{* \prime} s$ and $\mathcal{S}_{Q_{2}}{ }^{\prime} s$.

One advantage of this approach is that it can deal with general models for sen- 
sor placement, including non-linear and/or time-varying models as well as some complex models. Moreover, this approach directly uses FSM and SSM and thus is capable of coping with the sensor placement issue in such a system which has no numerical model but provides FSM and SSM. The main disadvantage indicates that this approach consumes computation on structurally analyzing and yielding one degree redundancy overconstrained subsystems. The consumed time increases exponentially as the structural redundancy $\bar{\varphi}$. The algorithm presented in [82] is one of the most efficient algorithms but still has order of $n^{\bar{\varphi}+1.5}$, where $n$ is the number of equations in a model. 


\section{Chapter 4}

\section{Sensor Placement for Intrinsic}

\section{Fault Isolability on the Bond}

\section{Graph}

This chapter handles sensor placement issues in the context of BG modelling. The intention is to decide what subset of BG junctions should be equipped with sensors on the BG. The decision is made by analyzing intrinsic fault detectability and isolability of faults. For fault detectability and isolability analysis, two simple conditions are provided that are constrained to be the subset of junctions for each fault. Using these two conditions, a novel sensor placement approach is designed to compute the optimal set of junctions (i.e., candidate sensors). In comparison with the SPLCDP in Chapter 3, this novel approach analyzes detectability and isolability in the image space of a DAEs model rather than a set of ARRs. This is one distinct feature of this approach. This feature makes this approach to obviate generating lots of useless ARRs.

This chapter is organized as follows. Section 4.1 defines a concept of the BG causality and also presents the fault set and the set of candidate sensors which 
are two primary ingredients for placing sensors on a BG. Section 4.2 attempts to present the reason why use causal paths for sensor placement. Section 4.3 shows a substructure representation which affects to trace back the occurrence of a fault and then makes two assignment rules of causal paths regarding to faults. Section 4.4 formulates a linear differential-algebraic equations (DAEs) model and investigates fault detectability and isolability in this model. Section 4.5 develops a sensor placement algorithm to steer towards what subset of junctions should be installed sensors. Section 4.6 studies two representative cases: an electrical circuit and a mechatronic system, to illustrate the performance of this new sensor placement approach and then validates the optimal solution by deriving specific ARRs. Section 4.7 brings the summary of this chapter.

\subsection{Faults and Sensor Locations on the Bond Graph}

This chapter considers all 1-port generic elements which have linear behaviors. For ease of establishment of a DAEs model, all constitutive relations for 1-port generic elements are transformed from the time domain into the Laplace domain. Consequently, the constitutive relations in the Laplace domain for all generic elements are summarized in Table 2.2. To avoid giving initial conditions of energy-storing BG elements, the derivative causality is preferable to be chosen when discussing the sensor placement issue. For ease of presentation in the remainder of this chapter, a terminological name for a BG in derivative causality is given as follows.

Definition 4.1. A differentially causalled bond graph (DCBG) is such a BG that all storage elements end up in derivative causality after the SCAP is performed.

On a DCBG, two generic BG elements are said to be causally connected if they exist a causal path. Thus, there are diverse types of causal connections on a DCBG, such as a type of connection between two 1-port elements, a type of connection 
between a 1-port element and a junction, and so forth. However, this chapter pays particular attention to one type of causal connection between the 1-port element and the junction for fault detectability and isolability. The reasons are stated as follows. In the BG modelling, 1-port BG elements model all devices except transducers in a system. During operation, devices (with the exception of transducers that are assumed normal in this paper) become faulty and thus 1-port BG elements are thought of as the possible faults. This chapter assumes that a BG has $Q$ faults and let $\mathcal{F}=\left\{f^{1}, f^{2}, \ldots, f^{Q}\right\}$ denote the set of these $Q$ faults. Mounting sensors on the locations in a system amounts to that sensors are directly attached to junctions on a BG. As s result, junctions are considered as all possible sensor locations. For notational convenience, let $\mathcal{J}=\left\{\mathbf{0}_{1}, \mathbf{0}_{2}, \ldots, \mathbf{0}_{K_{1}}, \mathbf{1}_{1}, \mathbf{1}_{2}, \ldots, \mathbf{1}_{K_{2}}\right\}$ denote the set of $K$ junctions where $K=K_{1}+K_{2}$. A logical next step is to explain why the causal paths are employed for sensor placement. In other words, the next step shows the motivation of this chapter.

\subsection{Motivation for Use of Casual Paths}

Before presenting the main objective of this paper, an electrical circuit example is used to illustrate the sensor placement analysis for fault diagnosability. The circuit example has been used in [89] to validate the performance of placing sensors in a linear DAEs model. This circuit example in Figure 4.1 consists of six components: a voltage source $V$, an internal resistor of the voltage source $r$, two resistors $R_{1}$ and $R_{2}$, an inductor $L$ and a capacitor $C$. This example considers six faults occurring in $\left\{V, r, C, R_{1}, R_{2}, L\right\}$, respectively. Accordingly, the corresponding fault set is $\mathcal{F}_{\text {e.g. }}=$ $\left\{f^{V}, f^{r}, f^{C}, f^{R_{1}}, f^{R_{2}}, f^{L}\right\}$. Execution of the sensor placement algorithm presented in [89] produces a resulting set of sensors $\left\{z_{1}, z_{2}\right\}$, where sensors $z_{1}, z_{2}$ are mounted to measure currents $i_{r}$ and $i_{L}$, respectively. System equations to describe the dynamics 


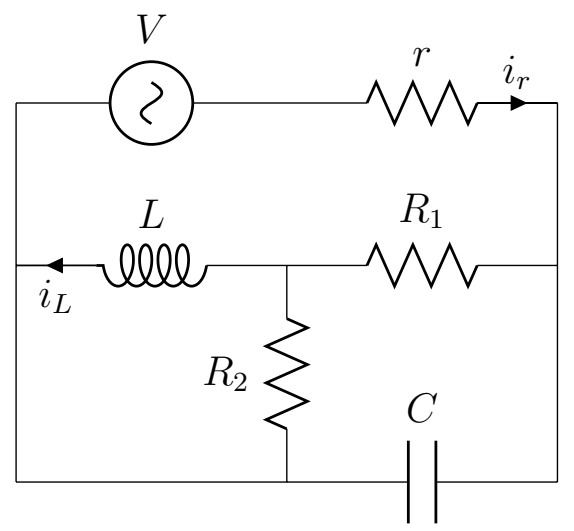

Figure 4.1: An circuit example

of this circuit with sensors $z_{1}, z_{2}$ are formulated according to Kirchhoff's laws. In these system equations, ARRs are generated by eliminating unknown variables and retaining known variables and fault signals. The generation of ARRs in [89] accounts for keeping different faults and sensors information. As a result, in this example with sensors $z_{1}, z_{2}$, a total of seven ARRs are produced and expressed by the following forms:

$$
\begin{gathered}
r_{1}=z_{1}-\frac{V-r z_{1}}{R_{1}}+\frac{L}{R_{1}} \dot{z}_{2}-C\left(\dot{V}-r \dot{z}_{1}\right) \\
r_{2}=z_{2}+L \dot{z}_{2}\left(\frac{1}{R_{1}}+\frac{1}{R_{2}}\right)-\frac{V-r z_{1}}{R_{1}} \\
r_{3}=z_{1}-z_{2}-\frac{L \dot{z}_{2}}{R_{2}}-C\left(\left(\frac{R_{1}}{R_{2}}+1\right) L \ddot{z}_{2}+R_{1} \dot{z}_{2}\right) \\
r_{4}=z_{1}-z_{2}-\frac{L \dot{z}_{2}}{R_{2}}-C\left(\dot{V}-r \dot{z}_{1}\right) \\
r_{5}=z_{1}-\frac{V-r z_{1}}{R_{1}}+\frac{R_{2}}{R_{1}}\left(z_{1}-z_{2}\right)-\left(\frac{R_{2}}{R_{1}}+1\right) C\left(\dot{V}-r \dot{z}_{1}\right) \\
r_{6}=V-r z_{1}-R_{1} z_{1}+\left(R_{1} C+\frac{L}{R_{2}}\right)\left(\dot{V}-r \dot{z}_{1}\right)-L\left(\frac{R_{1}}{R_{2}}+1\right)\left(\dot{z}_{1}-C\left(\ddot{V}-r \ddot{z}_{1}\right)\right) \\
r_{7}=\left(\frac{R_{1}}{R_{2}}+1\right) C L \ddot{z}_{2}+C R_{1} \dot{z}_{2}+\left(\frac{R_{1}}{r}+\frac{R_{2}}{r}+1\right) \frac{L}{R_{2}} \dot{z}_{2}+\left(\frac{R_{1}}{r}+1\right) z_{2}-\frac{V}{r} .
\end{gathered}
$$

It is not hard to see that any four of ARRs $r_{1}, \ldots, r_{5}$ can fulfill each pair in $\left\{\left(f^{i}, f^{j}\right) \in\right.$ $\left.\mathcal{F}_{\text {e.g. }} \times \mathcal{F}_{\text {e.g. }} \mid\left(f^{i}, f^{j}\right) \neq\left(f^{V}, f^{r}\right)\right\}$ distinguishable, by analyzing faults information involved in ARRs $r_{1}, \ldots, r_{7}$. From this point of view, the sensor set $\left\{z_{1}, z_{2}\right\}$ provided by $[89,90]$ is a feasible solution to the circuit example. However, after analyzing 
expressions $r_{1}, \ldots, r_{7}$, one can find that ARRs $r_{3}, \ldots, r_{7}$ are linear combinations of $r_{1}, r_{2}$ and their derivatives. Thus, ARRs $r_{3}, \ldots, r_{7}$ are elaborated in terms of $r_{1}, r_{2}$ as follows

$$
\begin{aligned}
& r_{3}=r_{1}-r_{2}-C R_{1} \dot{r}_{2} \\
& r_{4}=r_{1}-r_{2} \\
& r_{5}=\left(\frac{R_{2}}{R_{1}}+1\right) r_{1}-\frac{R_{2}}{R_{1}} r_{2} \\
& r_{6}=-R_{1} r_{1}+L \dot{r}_{2}-L\left(\frac{R_{1}}{R_{2}}+1\right) \dot{r}_{1} \\
& r_{7}=C R_{1} \dot{r}_{2}+\left(\frac{R_{1}}{r}+1\right) r_{2}-r_{1} .
\end{aligned}
$$

For ARRs $r_{3}, \ldots, r_{7}$, they can be separated into two groups. One group consisting of $\left\{r_{3}, r_{4}, r_{5}\right\}$ aims to eliminate faults; while the other containing $\left\{r_{6}, r_{7}\right\}$ intends to retract sensors. In [79], $r_{1}, r_{2}$ are called primary ARRs and $r_{3}, \ldots, r_{7}$ are called combined ARRs. A primary ARR is a basic and minimal representation of system's redundancy. Primary ARRs are determined by the number and location of installed sensors. They are the basis for FDI and reflect the structural property of a system. Hence, placing sensors to yield primary ARRs matches the discussion of detectability and isolability from the aspect of structural property. Conversely, combined ARRs are products of imposing designers' intentions on the basic redundancies of a system. For instance, in practical applications, these intentions include suppressing the influence of the model uncertainties, increasing the sensitivity to faults, etc. Additionally, the possibilities of combining primary ARRs are theoretically limitless. Therefore, combined ARRs are unable to represent purely the structural property. From the structural property (i.e., primary ARRs) viewpoint, the sensor set $\left\{z_{1}, z_{2}\right\}$ is not a solution to achieve distinguishability of each pair in $\left\{\left(f^{i}, f^{j}\right) \in \mathcal{F}_{\text {e.g. }} \times \mathcal{F}_{\text {e.g. }} \mid\left(f^{i}, f^{j}\right) \neq\left(f^{V}, f^{r}\right)\right\}$. This study concerns enough primary ARRs generated in order to achieve isolability.

Figure 4.2 shows the DCBG of this circuit example constructed using the pro- 


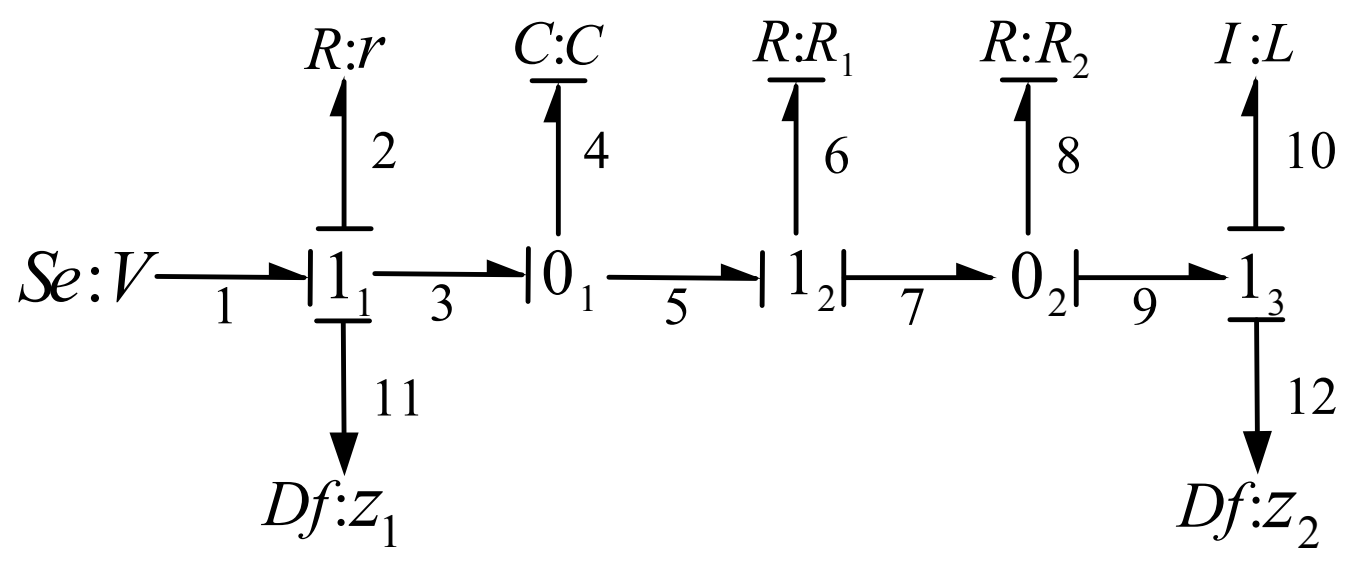

Figure 4.2: The DCBG of the circuit example

cedure for circuit construction [54]. On the DCBG, primary ARRs are referred to as independent ARRs in some literature [86,104,118]. In practice applications, they give rise to two advantages: (i) dependence on the model as little as possible and (ii) less computational burden from the implementation point. From the model-based FDI point of view, there is a well-known conclusion $[86,104,118]$ that the maximum number of primary ARRs is equal to the number of sensors. The derivation of primary ARRs on BGs demands the covering path method [111,119] which is a commonly-used derivation technique based on causal paths $[58,59,111,112]$. Using the ARR derivation technique, on the $\mathrm{BG}$ of this example with $z_{1}, z_{2}$ (shown in Figure 4.2), two primary ARRs $r_{1}, r_{2}$ can be derived from constitutive relations of junctions $\mathbf{0}_{1}, \mathbf{0}_{2}$. Thus, DCBGs, especially causal paths, play an important role in deriving primary ARRs. Additionally, using causal paths is a way of not generating combined ARRs and thus a means of preventing combined ARRs being used for fault diagnosability. Hence, motivated by these findings, this chapter develops a new sensor placement approach that considers cause-effect relationships of constitutive relations provided by causal paths, to ensure enough primary ARRs generated for isolability. A logical next step is to analyze causal paths for sensor placement. 
Chapter 4. Sensor Placement for Intrinsic Fault Isolability on the Bond Graph 81

\subsection{Causal Path Analysis}

The DCBG gives rise to algebraic loops [120] so as to solve the sensor placement issue in a problematic way. This section concentrates on analyzing the algebraic loops that are imposed on the causal path associated with a fault. From a BG point of view, the algebraic loop is a substructure of the DCBG where two 1-port elements have a closed causal path. For example, in Figure 4.2, BG elements $\left\{R_{2}, \mathbf{0}_{2}, \mathbf{1}_{3}, L\right\}$ form a closed causal path. This closed causal path is given by

$$
f_{9} \rightarrow \mathbf{1}_{3} \rightarrow f_{10} \rightarrow L \rightarrow e_{10} \rightarrow \mathbf{1}_{3} \rightarrow e_{9} \rightarrow \mathbf{0}_{2} \rightarrow e_{8} \rightarrow R_{2} \rightarrow f_{8} \rightarrow \mathbf{0}_{2} \rightarrow f_{9}
$$

Note that not all pairs of 1-port elements on a DCBG can form an algebraic loop. Among algebraic loops, some have impacts on sensor placement for fault detectability and isolability. With emphasis on these algebraic loops, specific definitions are made according to their structural characteristics. After that, two assignment rules of causal paths are made in the remainder of this section.

\subsubsection{Adjacently Connected Algebraic Loop}

From the viewpoint of algebra, an algebraic loop is where effort and flow variables depend on themselves in a substructure of the DCBG, i.e., these variables are solved in an endless loop of substitutions. In a BG sense, an algebraic loop indicates an effort and flow loop associated with a closed causal path. Two BG elements are said to be adjacently connected if the two elements are connected by a bond, transformer $(\rightarrow T F \rightarrow)$ or gyrator $(\rightarrow G Y \rightarrow)$. Following the above spirit, adjacently connected junctions denote two junctions that are linked by a bond, transformer or gyrator.

Definition 4.2. An adjacently connected algebraic loop (ACAL) is such an algebraic loop that one of the adjacently connected junctions connects to a 1-port BG element and the other ties up with another 1-port BG element. 


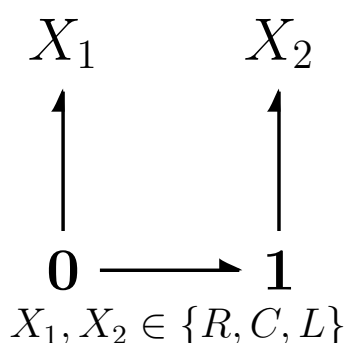

(a) ACAL
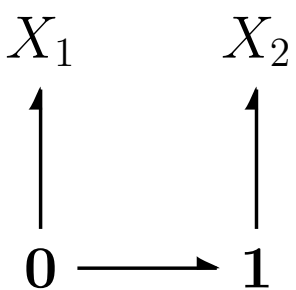

$X_{1} \in\{C, L\} X_{2} \in\{R, C, L\}$ or

$X_{1} \in\{R, C, L\} X_{2} \in\{C, L\}$

(b) ACDAL

Figure 4.3: ACALs where two junctions are connected by a bond.

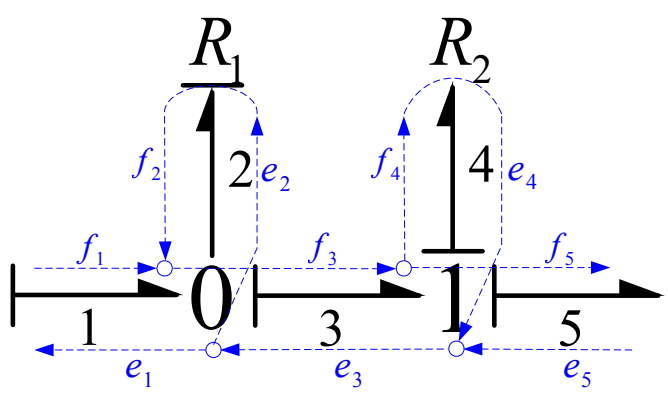

(a) An ACAL

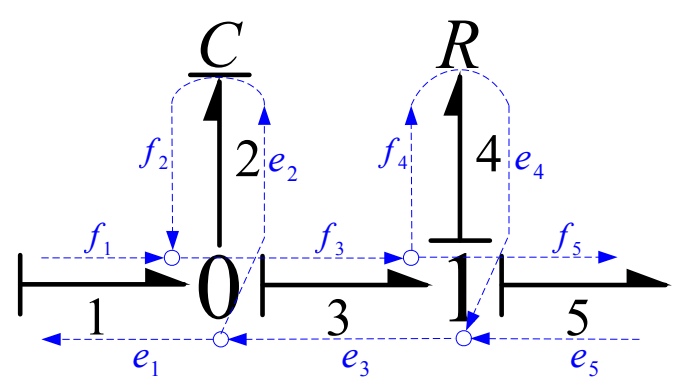

(b) An ACDAL

Figure 4.4: Two examples of ACALs

A general ACAL is shown in Figure 4.3(a) where $X_{1}$ as well as $X_{2}$ indicates any 1-port BG element in $\{R, C, L\}$. Consider $X_{1}=R_{1}$ and $X_{2}=R_{2}$ for example. The example diagram with bonds numbered is given in Figure 4.4(a). According to the constitutive relation(s) of every BG element, the following system of equations yields

$$
\left\{\begin{array}{l}
f_{1}=f_{2}+f_{3} \\
f_{2}=e_{2} / R_{1}=e_{3} / R_{1} \\
e_{3}=e_{4}+e_{5} \\
e_{4}=R_{2} f_{4}=R_{2} f_{3}
\end{array}\right.
$$


Chapter 4. Sensor Placement for Intrinsic Fault Isolability on the Bond Graph 83

Consider $f_{1}$ and $e_{5}$ as known variables. Solving the above equations yields

$$
\left[\begin{array}{cc}
\frac{1}{R_{1}} & 1 \\
1 & -R_{2}
\end{array}\right]\left[\begin{array}{l}
e_{3} \\
f_{3}
\end{array}\right]=\left[\begin{array}{l}
f_{1} \\
e_{5}
\end{array}\right]
$$

It is not hard to see that the value of each power variable in $\left\{e_{2}, f_{2}, e_{3}, f_{3}, e_{4}, f_{4}\right\}$ unique. Even if a single fault occurs on either $R_{1}$ or $R_{2}$, according to fault description in Section 2.4, values of these power variables are still unique but varied. In this ACAL, there is therefore no need to install a sensor to measure effort or flow variables for fault diagnosis since the abnormalities (i.e., sudden changes) of these power variables are only caused by faults. Next, consider another example with $X_{1}=C$ and $X_{2}=R$. The diagram corresponding to this example is shown in Figure 4.4(b). Similar to the above example, a system of equations can be generated from four BG elements $\{C, \mathbf{0}, \mathbf{1}, R\}$, which is

$$
\left\{\begin{array}{l}
f_{3}=f_{1}-f_{2} \\
f_{2}=C \dot{e}_{2}=C \dot{e}_{3} \\
e_{3}=e_{4}+e_{5} \\
e_{4}=f_{4} R=f_{3} R
\end{array} .\right.
$$

Solving it yields a non-homogeneous ODE in effort variable $e_{3}$ as follows

$$
C R \dot{e}_{3}+e_{3}=f_{1} R+e_{5}
$$

In (4.1), $f_{1}$ and $e_{5}$ are considered as known variables. Power variable $e_{3}$ in (4.1) depends on its initial condition and therefore has many values in either the faultfree or faulty modes. However, fault diagnosability requires every power variable with a unique value in order to trace back the root cause of its change. To achieve 

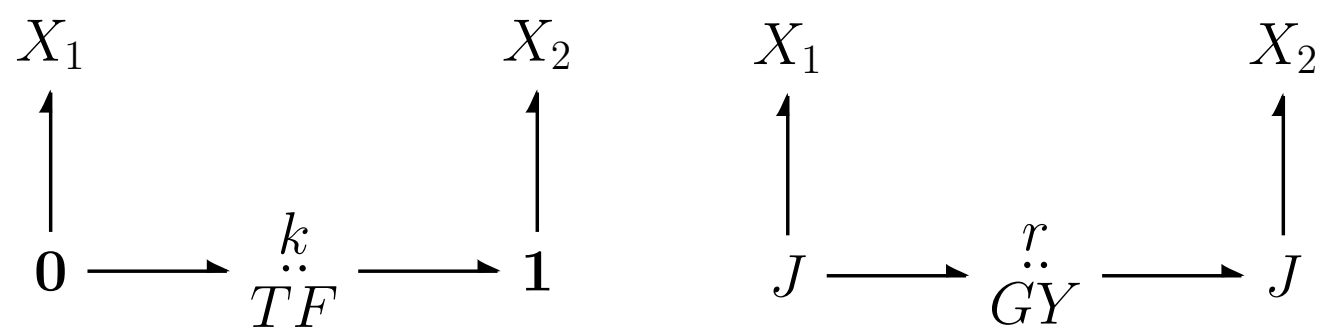

$X_{1} \in\{C, L\} X_{2} \in\{R, C, L\}$ or $X_{1} \in\{R, C, L\} X_{2} \in\{C, L\}$

$X_{1} \in\{C, L\} X_{2} \in\{R, C, L\}$ or $J \in\{\mathbf{0}, \mathbf{1}\}$

(a) An ACDAL where junctions are connected (b) An ACDAL where junctions are connected by a transformer $(\rightarrow T F \rightarrow)$.

by a gyrator $(\rightarrow G Y \rightarrow)$.

Figure 4.5: Two other general BG diagram structures of ACDALs

this, a junction of the ACAL must have a sensor to monitor the corresponding variable. In summary, these two examples of ACALs are different in the sense of fault diagnosability because the latter one needs a sensor, whilst the former does not. In order to distinguish these two substructures, a new definition is given to the substructure like the second example.

Definition 4.3. An adjacently connected differential algebraic loop (ACDAL) is defined as an ACAL if one of the adjacently connected junctions connects with a storage element, such as $C$ and $L$.

There are three types of BG diagram structures for ACDALs given in Figure 4.3(b), Figure 4.5(a) and Figure 4.5(b). These ACDALs belong to a kind of zero-order causal paths (ZCPs) [120].

\subsubsection{Assignment Rules of Causal Path for a Fault}

This chapter considers the causal path for fault $f^{i}$ which starts from fault $f^{i}$ and never passes through another faulty 1-port BG element, i.e., the causal path for fault $f^{i}$ only involves one fault. Let $\mathcal{X}_{C P}$ denote the set of power variables appeared on the causal path for fault $f^{i}$ and $\mathcal{X}_{A C D A L}$ the set of power variables appeared in an ACDAL. As the previous subsection stated, power variables in $\mathcal{X}_{A C D A L}$ have multiple 
values. On a DCBG, if the causal path for fault $f^{i}$ intersects with an ACDAL, the causal path must contain multi-valued power variables in $\mathcal{X}_{C P} \cap \mathcal{X}_{A C D A L}$ and moreover these multi-valued power variables propagate along the causal path. The existence of multi-valued power variables is harmful to trace back to fault $f^{i}$ (i.e., to monitor fault $f^{i}$ ) since it is hard to distinguish what changes of power variables are caused by fault $f^{i}$. Meanwhile, note that sensors are mounted on some junctions to prevent power variables having multiple values. For example, any junction of an ACDAL is installed by a sensor and thus the associated power variables are assigned into initial conditions, leading to their values to be unique. Therefore, for tracing back the occurrence of a fault, some rules are necessarily made to determine the length of the causal path for fault $f^{i}$ so that values of the associated power variables are unique with the installation of at most one sensor. In other words, to decide what subset of junctions can contribute to fault diagnosability.

Faults can be classified into two groups by the ACDAL. One group of faults occur on 1-port elements of the ACDAL, whereas the other group are those not of the ACDAL. The subsequent context discusses setting causal paths in two separate groups. First, one assignment rule is presented for fault $f^{i}$ occurring in an ACDAL element. As described in the previous subsection, two junctions in an ACDAL, if anyone is equipped with a sensor, ensure the associated power variables with unique values. This is beneficial to trace back to fault $f^{i}$. In addition to these two junctions, another junction adjacent to this ACDAL is impossible to make the associated power variables to have unique values, but potential to distinguish a fault in this ACDAL from another fault. Therefore for fault distinguishability, the causal path for a fault (occurring in an ACDAL element) should stretch to one adjacent junction of this ACDAL if possible. In summary, the casual path assignment rule for faults occurring in ACDAL elements is given as follows.

Rule 4.1. For a fault occurring in an ACDAL element, its causal path passes 
through two junctions of this ACDAL and then extends to one adjacent junction of this ACDAL if possible.

Using this rule to fault $f^{L}$ in Figure 4.2 (which belongs to the ACDAL between $R_{2}$ and $L$ ) gives rise to the causal path $L\left(f^{L}\right) \rightarrow e_{10} \rightarrow \mathbf{1}_{3} \rightarrow e_{9} \rightarrow \mathbf{0}_{2} \rightarrow e_{7} \rightarrow \mathbf{1}_{2}$.

Note that a source element (such as $S e, S f$ ) never forms an ACDAL with a storage element $C$ or $L$ connected with the adjacently connected junction since the causal path between these two BG elements is not closed. For example, the causal path between $V$ and $C$ in Figure 4.2 is $V \rightarrow e_{1} \rightarrow \mathbf{1}_{1} \rightarrow e_{3} \rightarrow \mathbf{0}_{1} \rightarrow e_{4} \rightarrow C \rightarrow f_{4} \rightarrow$ $\mathbf{0}_{1} \rightarrow f_{3} \rightarrow \mathbf{1}_{1} \rightarrow f_{1}$, which is open. Thus, the other causal path assignment rule for faults not occurring any ACDAL is made in the following.

Rule 4.2. For any fault $f^{i}$ not occurring in ACDALs, its causal path, if intersecting with less than two ACDALs, terminates at the last junction along this causal path; otherwise the causal path, if intersecting with two or more ACDALs, terminates at the second junction of the second $A C D A L$.

After applying the above two assignment rules on a DCBG, the DCBG exhibits all possible causal paths with respect to faults in $\mathcal{F}$ for detectability and isolability. To the circuit example, the resulting causal paths regarding faults in $\mathcal{F}_{\text {e.g. }}$ are drawn in Figure 4.6. For instance, in Figure 4.6, a causal path with respect to fault $f^{V}$ is arbitrarily chosen which is

$$
f^{V} \rightarrow e_{1} \rightarrow \mathbf{1}_{1} \rightarrow e_{3} \rightarrow \mathbf{0}_{1} \rightarrow e_{5} \rightarrow \mathbf{1}_{2}
$$

On the causal path (4.2), the relevant junctions are $\left\{\mathbf{1}_{1}, \mathbf{0}_{1}, \mathbf{1}_{2}\right\}$. From the sensor placement viewpoint, the useful elements on a causal path are junctions. Therefore, for simplicity's sake, it is desirable to convert the causal path into a set of junctions. Let $\mathcal{J}_{i}(\subseteq \mathcal{J})$ denote the subset of junctions with respect to fault $f^{i}$ in $\mathcal{F}$. On the DCBG of the circuit example, through counting junctions along causal paths, 
Chapter 4. Sensor Placement for Intrinsic Fault Isolability on the Bond Graph 87

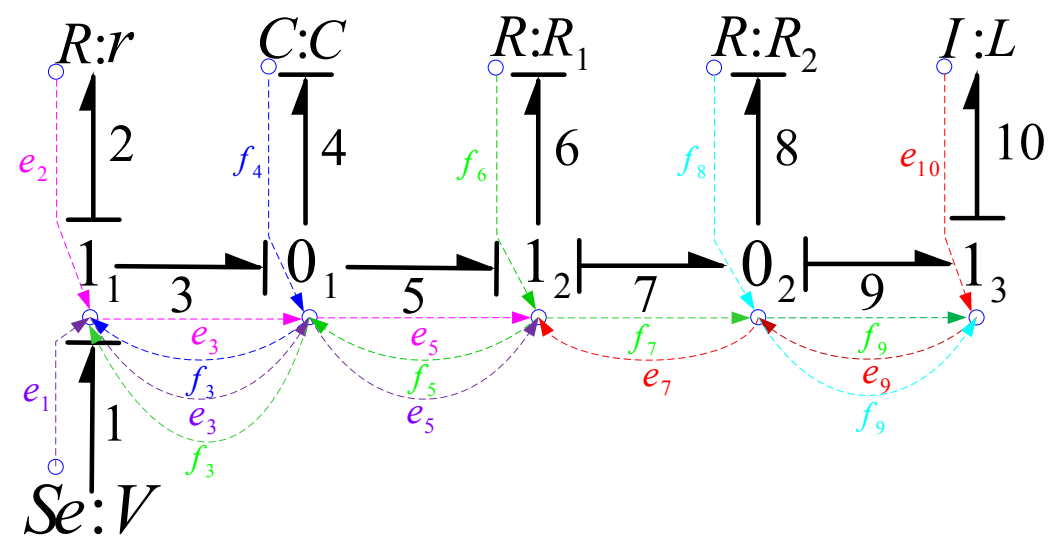

Figure 4.6: The DCBG with six causal paths.

six subsets of junctions with respect to faults in $\mathcal{F}_{\text {e.g. }}$ are $\mathcal{J}_{V}=\left\{\mathbf{1}_{1}, \mathbf{0}_{1}, \mathbf{1}_{2}\right\}, \mathcal{J}_{r}=$ $\left\{\mathbf{1}_{1}, \mathbf{0}_{1}, \mathbf{1}_{2}\right\}, \mathcal{J}_{C}=\left\{\mathbf{1}_{1}, \mathbf{0}_{1}\right\}, \mathcal{J}_{R_{1}}=\left\{\mathbf{1}_{1}, \mathbf{0}_{1}, \mathbf{1}_{2}, \mathbf{0}_{2}, \mathbf{1}_{3}\right\}, \mathcal{J}_{R_{2}}=\left\{\mathbf{0}_{2}, \mathbf{1}_{3}\right\}$ and $\mathcal{J}_{L}=\left\{\mathbf{1}_{2}, \mathbf{0}_{2}, \mathbf{1}_{3}\right\}$.

\subsection{Detectability and Distinguishability Analysis}

This section builds a linear differential-algebraic equations (DAEs) model using constitutive relations of a DCBG. Based on this DAEs model, this section also discusses some basic properties such as fault detectability and fault isolability. The presentation in [121] that utilizes the same type of models as what is used in the behavioral approach to systems theory [122] is the basic theory of this work. The basic theory is extended by incorporating the junction sets with respect to each fault in $\mathcal{F}$ into, in order to handle the sensor placement issue of a DCBG.

\subsubsection{A DAEs Model}

By assembling the constitutive relations for a DCBG and equations for fault description, the linear DAEs model of the DCBG is written as

$$
B(s) x_{1}+L^{\prime}(s) z+F^{\prime}(s) f=\mathbf{0},
$$


where $f$ is a vector of fault signals (i.e., $f=\left[\Delta_{1}, \Delta_{2}, \ldots, \Delta_{Q}\right]^{\top}$ ) and $z$ is a vector of inputs and outputs of the BG. These inputs and outputs are mentioned as known variables in the fault diagnosis field and also called observations in control theory. Due to fault description in Section $2.4, F^{\prime}(s)$ in (4.3) is actually an integer matrix that is composed of the elements 0 and -1 . The constitutive relations in a junction show that if a sensor is installed, the number of power variables measured by this sensor is equal to the number of bonds connected to this junction. However, this violates the convention that one possible sensor measures a single variable. To obey this convention, in each junction, a sensor variable $y_{k}$ (which is a unknown dummy variable) is introduced to define relations with power variables of this junction and thus (4.3) needs to be rewritten by adding these relations. Take a 1 -type junction with $l$ bonds for example. This 1-type junction provides the following $l$ equalized relations

$$
\begin{gathered}
y_{k}=f_{1} \\
y_{k}=f_{2} \\
\vdots \quad \vdots \\
y_{k}=f_{l},
\end{gathered}
$$

where $y_{k}$ is a sensor variable for this 1 -type junction and $f_{1}, f_{2}, \cdots f_{l}$ its flow variables. All equations like (4.4) that describe relations of $y_{k}(k=1,2, \cdots, K)$ in each junction, are integrated with (4.3) to yield a new linear DAEs

$$
H(s) x+L(s) z+F(s) f=\mathbf{0},
$$

where $x$ represents a new vector $\left[x_{1}^{\top}, y_{1}, y_{2}, \cdots, y_{K}\right]^{\top}, L(s)=\left[\begin{array}{c}L^{\prime}(s) \\ \mathbf{0}\end{array}\right]$ and $F(s)=$ $\left[\begin{array}{c}F^{\prime}(s) \\ \mathbf{0}\end{array}\right]$. For $H(s)$ in $(4.5)$, as noted in Section 4.1, there exists an elementary 
matrix $U(s)$ such that

$$
U(s) H(s)=\left[\begin{array}{cc}
B(s) & \mathbf{0} \\
\Gamma & I_{K} \\
\mathbf{0} & \mathbf{0}
\end{array}\right]
$$

where $I_{K}$ is a $K \times K$ identity matrix and $\Gamma$ denotes a matrix with size of $2 n \times K$. Apparently, matrix $H(s)$ has full row rank.

Note that a fault signal $\Delta_{i}$ in vector $f$ represents the bias of a power variable of a faulty 1-port element. Then, for any fault signal $\Delta_{i}$ in a DAEs model, there exists vectors $x$ and $z$ consistent with the model (4.5), i.e., this model imposes no restriction on any feasible $\Delta_{i}$. Formally, this is identical to that for all columns $F_{i}(s)$ in $F(s)$, it holds that

$$
F_{i}(s) \in \operatorname{Im}[H(s) L(s)]
$$

On the other hand, the sensor variable $y_{k}$ can appear to be an embodiment of a junction since each junction has only one sensor variable. Following this finding, a new notation $\mathcal{P}$ is defined as the set of sensor variables, it can be directly obtained by substituting each junction in $\mathcal{J}$ with the corresponding sensor variable $y_{k}$ in sequence. Similar to the set $\mathcal{P}$, the subset $\mathcal{P}_{i}\left(\mathcal{P}_{i} \subseteq \mathcal{P}\right)$ with respect to fault $f^{i}$ is straightforwardly generated by the replacement of all junctions in $\mathcal{J}_{i}$. For a clear understanding of subset $\mathcal{P}_{i}$, six subsets with respect to faults in $\mathcal{F}_{\text {e.g. }}$ are exemplified. These subsets of sensor variables are $\mathcal{P}_{V}=\left\{y_{1}, y_{2}, y_{3}\right\}, \mathcal{P}_{r}=\left\{y_{1}, y_{2}, y_{3}\right\}, \mathcal{P}_{C}=$ $\left\{y_{1}, y_{2}\right\}, \mathcal{P}_{R_{1}}=\left\{y_{1}, y_{2}, y_{3}, y_{4}, y_{5}\right\}, \mathcal{P}_{R_{2}}=\left\{y_{4}, y_{5}\right\}$ and $\mathcal{P}_{L}=\left\{y_{3}, y_{4}, y_{5}\right\}$.

\subsubsection{Basic Properties of Detectability and Distinguishabil- ity}

Before presenting two concepts of fault detectability and fault distinguishability, a new concept of the sensor binary vector is introduced for ease of description. 
Definition 4.4. A sensor binary vector $\Theta_{\mathrm{sb}}\left(y_{k}\right)$ is a binary row-vector with size $2 n+K$ where all elements are zeros except one element that lies at the $(2 n+k)$-th position in $x$ (i.e., at the position of $y_{k}$ in $x$ ).

For instance, in the circuit example, $x=\left[x_{1}^{\top}, y_{1}, y_{2}, y_{3}, y_{4}, y_{5}\right]^{\top}$ where $x_{1}$ contains 20 power variables; $y_{1}$ is the 21-st unknown variable in $x$; then $\Theta_{\mathrm{sb}}\left(y_{1}\right)$ is a binary vector where all are zeros but the 21-st entry (which is one). On DCBGs, when a sensor is connected with a junction through a bond, i.e., a sensor equation $y_{k}=z_{\text {new }}$ $\left(y_{k} \in \mathcal{P}\right)$ is added into model (4.5), the resulting model is expressed as

$$
\left[\begin{array}{c}
H(s) \\
\Theta_{\mathrm{sb}}\left(y_{k}\right)
\end{array}\right] x+\left[\begin{array}{cc}
L(s) & \mathbf{0} \\
\mathbf{0} & -1
\end{array}\right]\left[\begin{array}{c}
z \\
z_{\text {new }}
\end{array}\right]+\left[\begin{array}{c}
F(s) \\
\mathbf{0}
\end{array}\right] f=\mathbf{0}
$$

One must find that (4.7) can be written as the form of (4.5) as long as the corresponding matrices are redefined. Therefore, in the sequel of this chapter, the DAEs model (4.5) represents a DCBG where sensors are either installed or not.

These two concepts are presented from the viewpoint of set theory. The set of observations is directly defined using the behavioral approach [122] that is consistent with various behavioral modes. For instance, the set of observations consistent with the faultless model is expressed as

$$
\mathcal{O}(N F)=\{z \mid \exists x: H(s) x+L(s) z=\mathbf{0}\}
$$

The observations consistent with a behavioral mode of fault $f^{i}$ are those where there exists a fault signal $\Delta_{i}$ representing the occurrence of fault $f^{i}$, a vector of unknown signals $x$ and a sensor variable $y_{k} \in \mathcal{P}_{i}$ such that the model in (4.5) is consistent 
Chapter 4. Sensor Placement for Intrinsic Fault Isolability on the Bond Graph 91

and furthermore restricted by $H_{m}^{r}(s)=\Theta_{\mathrm{sb}}\left(y_{k}\right)$, i.e.,

$$
\begin{aligned}
& \mathcal{O}\left(f^{i}\right)=\left\{z \mid \exists x, \Delta_{i}, y_{k} \in \mathcal{P}_{i} \text { and } m \in N:\right. \\
& \left.H(s) x+L(s) z+F_{i}(s) \Delta_{i}=\mathbf{0} \wedge H_{m}^{r}(s)=\Theta_{\mathrm{sb}}\left(y_{k}\right)\right\},
\end{aligned}
$$

where $H_{m}^{r}(s)$ denotes the $m$-th row of matrix $H(s)$. With the help of these sets of observations $\mathcal{O}(N F)$ and $\mathcal{O}\left(f^{i}\right)$, a definition on detectability is smoothly made.

Definition 4.5 ( [89]). Fault $f^{i}$ is detectable in (4.5) if $\mathcal{O}\left(f^{i}\right) \nsubseteq \mathbb{O}(N F)$.

Although intuitive, a condition of fault detectability that is directly related to the model matrices restricted by the set of sensor variables $\mathcal{P}_{i}$ with respect to fault $f^{i}$ is given as follows.

Theorem 4.1. Fault $f^{i}$ is detectable in (4.5) iff $F_{i}(s) \notin \operatorname{Im}[H(s)]$ such that $\exists y_{k} \in$ $\mathcal{P}_{i}$ and $m \in \mathbb{Z}^{+}, H_{m}^{r}(s)=\Theta_{\mathrm{sb}}\left(y_{k}\right)$.

Proof. Fault $f^{i}$ is detectable iff there is a residual that contains fault $f^{i}$. Generating such a residual has been depicted in [121]. In principle, a residual generator assures that when decoupling the unknown variables $x$, fault $f^{i}$ should not also be decoupled. For $H(s) x+L(s) z+F_{i}(s) \Delta_{i}=\mathbf{0}$, the necessary and sufficient condition to yield a residual is that there exists a matrix $N_{H}(s)$, satisfying $N_{H}(s) H(s)=\mathbf{0}$ and $N_{H}(s) F_{i}(s) \neq \mathbf{0}$. Solving these two conditions yields $\operatorname{Im}[H(s)] \subseteq \operatorname{Right}-\operatorname{Ker}\left[N_{H}(s)\right]$ and $F_{i}(s) \notin$ Right-Ker $\left[N_{H}(s)\right]$. Hence, for $H(s) x+L(s) z+F_{i}(s) \Delta_{i}=\mathbf{0}$, fault $f^{i}$ is detectable iff $F_{i}(s) \notin \operatorname{Im}[H(s)]$, which has been proved in $[35,75,89,121]$. Additionally, matrix $H(s)$ is subject to a constraint that $\exists y_{k} \in \mathcal{P}_{i}$ and $m \in \mathbb{Z}^{+}, H_{m}^{r}(s)=\Theta_{\mathrm{sb}}\left(y_{k}\right)$. This ends the proof of Theorem 4.1.

This theorem seems more straightforward since it does not include the notion of the residual generator. Simultaneously, it is attractive that fault detectability is defined in terms of model properties other than properties of a set of ARRs. 


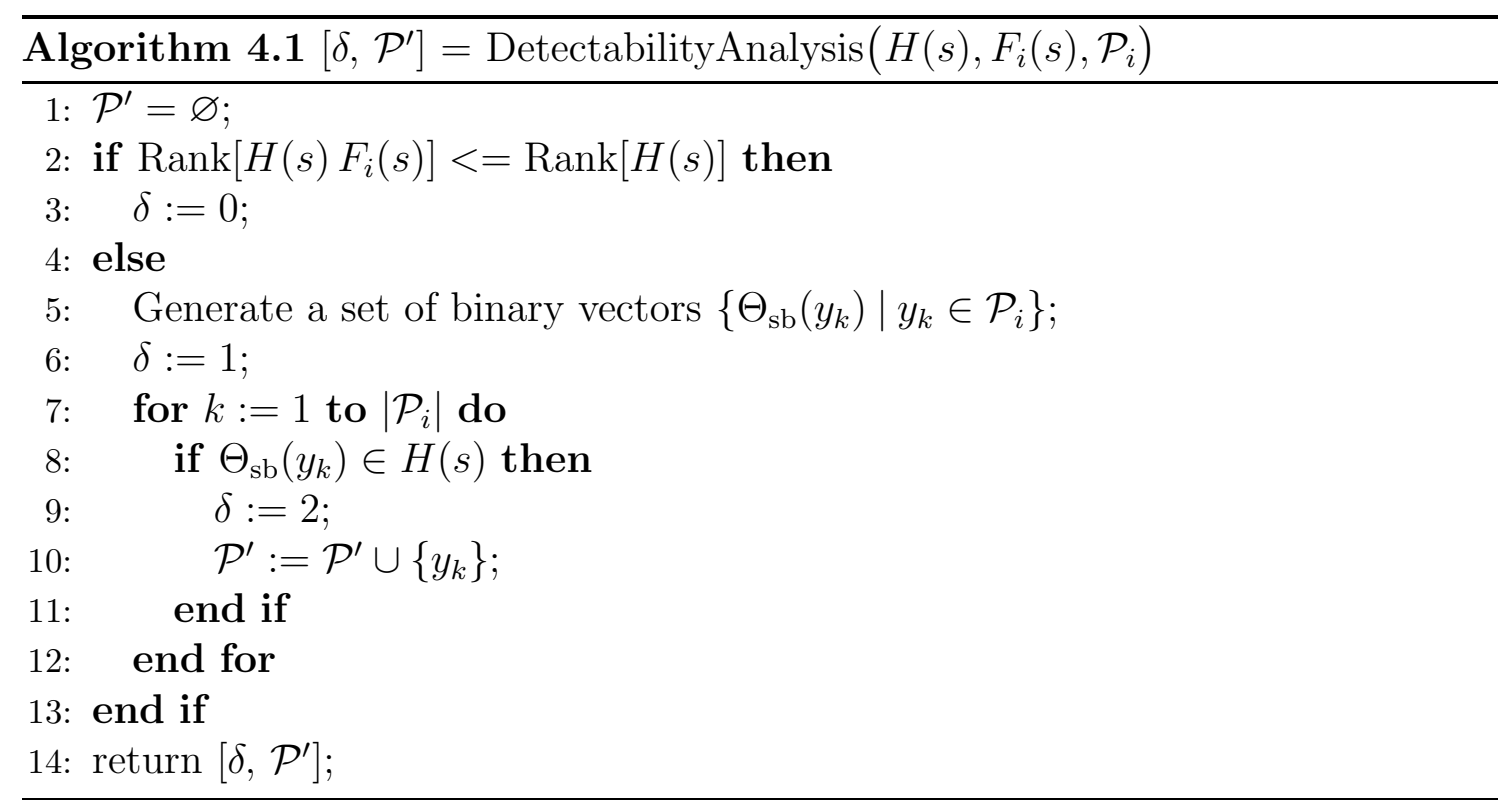

Note that $F_{i}(s) \notin \operatorname{Im}[H(s)]$ can be performed by a rank test. It then follows that $\operatorname{Rank}\left[H(s) F_{i}(s)\right]>\operatorname{Rank}[H(s)]$. From a computational viewpoint, the rank test is simpler.

Using the above theorem to inspect a fault, there are usually two states, i.e., detectable and non-detectable. However, the non-detectable state is caused by two cases (i) $F_{i}(s) \in \operatorname{Im}[H(s)]$ and (ii) $F_{i}(s) \notin \operatorname{Im}[H(s)]$ but $\nexists y_{k} \in \mathcal{P}_{i}$. For the sake of clarity, the state $\delta$ is encoded as an integer and thus the set $\{0,1,2\}$ is employed to indicate the value domain of $\delta$, i.e., $\delta \in\{0,1,2\}$, where 0 denotes case (i), 1 represents case (ii) and 2 means the detectable state. Given a matrix $H(s)$, a vector $F_{i}(s)$ and a subset $\mathcal{P}_{i}$ with respect to fault $f^{i}$, the pseudo-code to check detectability of fault $f^{i}$ is presented in the following.

Detectability is a special form of distinguishability. More specifically, a fault is detectable if this fault is distinguishable from the fault-free mode. By noticing this similarity, it is natural to state the following definition.

Definition 4.6 ( [89]). Fault $f^{i}$ is distinguishable from fault $f^{j}$ in (4.5) if $\mathcal{O}\left(f^{i}\right) \nsubseteq$ $\mathcal{O}\left(f^{j}\right)$. 
Chapter 4. Sensor Placement for Intrinsic Fault Isolability on the Bond Graph 93

In an analogous way to detectability, a condition of fault distinguishability that pertains to the model matrices constrained by subsets $\mathcal{P}_{i}, \mathcal{P}_{j}$ with respect to two faults $f^{i}, f^{j}$ is given in the below.

Theorem 4.2. Fault $f^{i}$ is distinguishable from fault $f^{j}$ in (4.5) iff $F_{i}(s) \notin \operatorname{Im}\left[H(s) F_{j}(s)\right]$ such that $\exists y_{k} \in \mathcal{P}_{i}, y_{k^{\prime}} \in \mathcal{P}_{j}\left(y_{k} \neq y_{k^{\prime}}\right)$ and $m, m^{\prime} \in \mathbb{Z}^{+}, H_{m}^{r}(s)=\Theta_{\mathrm{sb}}\left(y_{k}\right)$ and $H_{m^{\prime}}^{r}(s)=\Theta_{\mathrm{sb}}\left(y_{k^{\prime}}\right)$.

Proof. It follows from Definition 4.6 that fault $f^{j}$ is detectable. To a detectable fault $f^{j}$, the set of observations can be written by

$$
\begin{aligned}
& \mathcal{O}\left(f^{j}\right)=\left\{z \mid \exists x, \Delta_{j}, y_{k^{\prime}} \in \mathcal{P}_{j} \text { and } m^{\prime} \in \mathbb{Z}^{+}:\right. \\
& \left.\left[H(s) F_{j}(s)\right]\left(\begin{array}{c}
x \\
\Delta_{j}
\end{array}\right)+L(s) z=\mathbf{0} \wedge \underline{H_{m^{\prime}}^{r}(s)=\Theta_{\mathrm{sb}}\left(y_{k^{\prime}}\right)}\right\} .
\end{aligned}
$$

In (4.10), the matrix $H(s)$ is subject to the constraint that $\exists y_{k^{\prime}} \in \mathcal{P}_{j}, m^{\prime} \in \mathbb{Z}^{+}, H_{m^{\prime}}^{r}(s)=$ $\Theta_{\mathrm{sb}}\left(y_{k^{\prime}}\right)$. Let the symbol $f_{N C}^{j}$ denote fault $f^{j}$ whose restriction of junctions (i.e., sensor variables) are not considered for detectability. Then, the set of observations with respect to fault $f_{N C}^{j}$ is of the following form

$$
\mathcal{O}\left(f_{N C}^{j}\right)=\left\{z \mid \exists x, \Delta_{j}:\left[H(s) F_{j}(s)\right]\left(\begin{array}{c}
x \\
\Delta_{j}
\end{array}\right)+L(s) z=\mathbf{0}\right\} .
$$

Note that (4.11) has the same form as (4.8) where $H(s)$ is substituted by $\left[H(s) F_{j}(s)\right]$. It follows from Definition 4.5 that fault $f^{i}$ is distinguishable from fault $f_{N C}^{j}$ if $\mathcal{O}\left(f^{i}\right) \nsubseteq \mathcal{O}\left(f_{N C}^{j}\right)$. Therefore, implied by the condition of detectability, the condition of distinguishing fault $f^{i}$ from fault $f_{N C}^{j}$ is that $F_{i}(s) \notin \operatorname{Im}\left[H(s) F_{j}(s)\right]$ and there exists a sensor variable $y_{k} \in \mathcal{P}_{i}\left(y_{k} \neq y_{k^{\prime}}\right)$ to restrict matrix $H(s)$, i.e.,

$$
\begin{aligned}
& F_{i}(s) \notin \operatorname{Im}\left[H(s) F_{j}(s)\right] \\
& \text { s.t. } \exists y_{k} \in \mathcal{P}_{i}\left(y_{k} \neq y_{k^{\prime}}\right), m \in \mathbb{Z}^{+}, H_{m}^{r}(s)=\Theta_{\mathrm{sb}}\left(y_{k}\right) .
\end{aligned}
$$


By comparing (4.10) with (4.11), the set $\mathcal{O}\left(f^{j}\right)$ is the set $\mathcal{O}\left(f_{N C}^{j}\right)$ which is constrained by the underlined in (4.10). Apparently, $\mathcal{O}\left(f^{j}\right) \subset \mathcal{O}\left(f_{N C}^{j}\right)$. Straightforwardly, $\mathcal{O}\left(f^{i}\right) \nsubseteq \mathcal{O}\left(f^{j}\right)$ under the condition (4.12). By summarizing constraints imposed on matrix $H(s)$ in (4.10) and (4.12), the condition of distinguishing faults $f^{i}$ from $f^{j}$ follows. This ends the proof.

Note that two arbitrary faults, if one is distinguishable from a second fault, then the second fault is certainly distinguishable from the first. Distinguishability of two faults is a symmetric relation. As for the rank form of detectability, deriving the rank form for distinguishability is attempted. $F_{i}(s) \notin\left[H(s) F_{j}(s)\right]$ can be expressed in a rank manner as $\operatorname{Rank}\left[H(s) F_{i}(s) F_{j}(s)\right]>\operatorname{Rank}\left[H(s) F_{j}(s)\right]$. Due to $F_{j}(s) \notin H(s)$, it follows that $\operatorname{Rank}\left[H(s) F_{j}(s)\right]=\operatorname{Rank}[H(s)]+1$. Therefore, $F_{i}(s) \notin\left[H(s) F_{j}(s)\right]$ can performed as $\operatorname{Rank}\left[H(s) F_{i}(s) F_{j}(s)\right]-\operatorname{Rank}[H(s)] \geq 2$.

Similar to Theorem 4.1, this theorem also owns three states and their value domain are defined as $\{0,1,2\}$. Given a matrix $H(s)$, vectors $F_{i}(s), F_{j}(s)$ and subsets $\mathcal{P}_{i}, \mathcal{P}_{j}$ of two faults $f^{i}, f^{j}$, the following pseudo-code is used to distinguish a pair of faults $f^{i}, f^{j}$.

\subsection{Sensor Placement Analysis}

This section solves sensor placement issue on DCBGs in a theoretical and algorithmic way. This section follows the framework of sensor placement analysis presented in Section 4 of [89]. When discussing the sensor placement issue, the example circuit is used as a running example to illustrate the relevant results. The section is organized according to different diagnosability requirements. Section 4.5.1 achieves the maximum detectability, Section 4.5.2 the maximum isolability and Section 4.5.3 the maximum detectability and isolability. Before presenting these subsections, it is necessary to define the optimal set of sensors for the achievement of a requirement. 


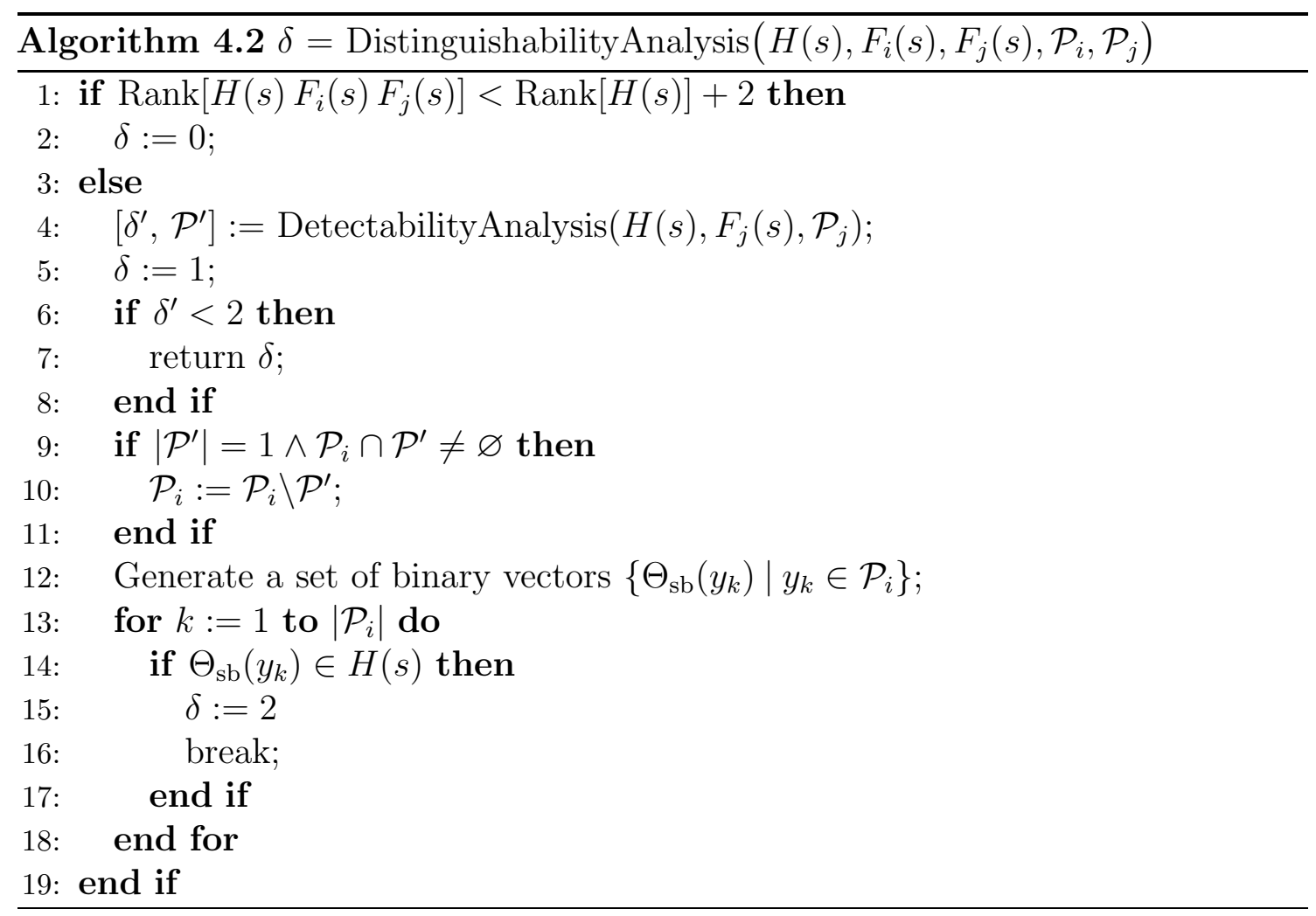

Definition 4.7 (Minimum Sensor Set). Let $\mathcal{P}$ be the set of sensor variables (i.e., the embodied set of sensor locations) and let $\mathcal{S}$ be a set of sensors defined on $\mathcal{P}$. Sensors in $\mathcal{S}$ are no repetition. Given a requirement of maximum detectability and/or maximum isolability, $\mathcal{S}$ is called a minimum sensor set if the requirement is fulfilled when all sensors in $\mathcal{S}$ are added, while not fulfilled when those sensors in any proper subset of $\mathcal{S}$ are added.

\subsubsection{Sensor Placement for Detectability}

Recall that $H(s)$ in (4.5) has full rank. A basic building block of the sensor placement algorithm is to seek minimum sensor sets that achieve maximum detectability of faults in $\mathcal{F}$. In the basic building block, the critical step is to determine what subset of sensors should be added. This step is explicated by the following lemma in a constructive and algorithmic way. 
Lemma 4.1. Let $f^{i} \in \mathcal{F}$ be a non-detectable fault, $\mathcal{P}_{i}$ the subset of sensor variables (which are unknown) with respect to fault $f^{i}$, and $Y(s)$ the unique solution to

$$
H(s) Y(s)=F_{i}(s)
$$

Then fault $f^{i}$ becomes detectable iff any sensor variable $y_{k}$ in the set $\left\{y_{k} \in \mathcal{P}_{i} \mid Y_{2 n+k}(s) \neq\right.$ $0\}$ is measured.

Proof. With the sensor equation $z_{\text {new }}=y_{k}\left(y_{k} \in \mathcal{P}_{i}\right)$ added, it is not hard to see that an extended DAEs model in form of (4.7) also fulfills condition (4.6). According to Theorem 4.1, fault $f^{i}$ becomes detectable iff $\left[\begin{array}{c}F_{i}(s) \\ 0\end{array}\right] \notin\left[\begin{array}{c}H(s) \\ \Theta_{\mathrm{sb}}\left(y_{k}\right)\end{array}\right]$ such that $y_{k} \in \mathcal{P}_{i}$. Therefore, fault $f^{i}$ is detectable iff there is no solution $Y(s)$ to the following equations

$$
\begin{aligned}
& H(s) Y(s)=F_{i}(s) \\
& \Theta_{\mathrm{sb}}\left(y_{k}\right) Y(s)=0
\end{aligned}
$$

where $y_{k} \in \mathcal{P}_{i}$. Since $Y(s)$ is the unique solution of (4.13), $\Theta_{\mathrm{sb}}\left(y_{k}\right) Y(s) \neq 0$, i.e., $Y_{2 n+k} \neq 0\left(y_{k} \in \mathcal{P}_{i}\right)$. This inequality holds iff any sensor variable $y_{k}$ in the set $\left\{y_{k} \in \mathcal{P}_{i} \mid Y_{2 n+k}(s) \neq 0\right\}$ is measured. This proves Lemma 4.1.

As a result of Lemma 4.1, the detectability set with respect to $f^{i}$ has the form

$$
\mathfrak{D}\left(f^{i}\right)=\left\{y_{k} \in \mathcal{P}_{i} \mid Y_{2 n+k} \neq 0 \wedge H(s) Y(s)=F_{i}(s)\right\} .
$$

For a non-detectable fault $f^{i}$, its detectability is achieved if $\mathfrak{D}\left(f^{i}\right)$ is nonempty and any sensor variable in this set is measured. For the other case of $\mathfrak{D}\left(f^{i}\right)=\varnothing$, there are two possibilities. One is that fault $f^{i}$ is impossible to fulfill its detectability and the other is that fault $f^{i}$ is already detectable because $H(s) Y(s)=F_{i}(s)$ has no solution to the detectable fault $f^{i}$. One may find that Lemma 4.1 only characterizes 
Chapter 4. Sensor Placement for Intrinsic Fault Isolability on the Bond Graph 97

a part of faults (such that $\mathfrak{D}\left(f^{i}\right) \neq \varnothing$ ) to achieve the detectability. In order to accomplish maximum detectability on a fault set $\mathcal{F}$, the following theorem is thus formalized.

Theorem 4.3. Let $\mathcal{F}$ be the set of faults in the linear DAEs model $M, \mathbb{P}$ the family of subsets $\mathcal{P}_{i}^{\prime}$ s and $M_{S}$ the equations pertaining to adding all sensors in a set $\mathcal{S}$. Then maximum detectability on the set $\mathcal{F}$ in the model $M \cup M_{S}$ is gained iff to each set $\mathfrak{D}\left(f^{i}\right)$ (such that $\left.\mathfrak{D}\left(f^{i}\right) \neq \varnothing, f^{i} \in \mathcal{F}\right)$, its intersection with $\mathcal{S}$ is nonempty.

Proof. Fault $f^{i}$ with $\mathfrak{D}\left(f^{i}\right)=\varnothing$ is either detectable without sensors added or not detectable with all additional sensors added. Hence, the maximum detectability is attained if all faults having nonempty detectability sets are made detectable by adding sensors in $\mathcal{S}$. Therefore from Lemma 4.1, the maximum detectability is achieved iff $\mathcal{S} \cap \mathfrak{D}\left(f^{i}\right) \neq \varnothing$ for all nonempty detectability sets $\mathfrak{D}\left(f^{i}\right)^{\prime} s$.

The results of the above theorem can be recapitulated by a family of detectability sets. The following pseudo-code is designed to compute this family $\mathbb{D}$, given a model $M$, a set of faults $\mathcal{F}$ and the family $\mathbb{P}$ of $\mathcal{P}_{i}^{\prime} s$ where each member is a subset with respect to fault $f^{i}$ in $\mathcal{F}$.

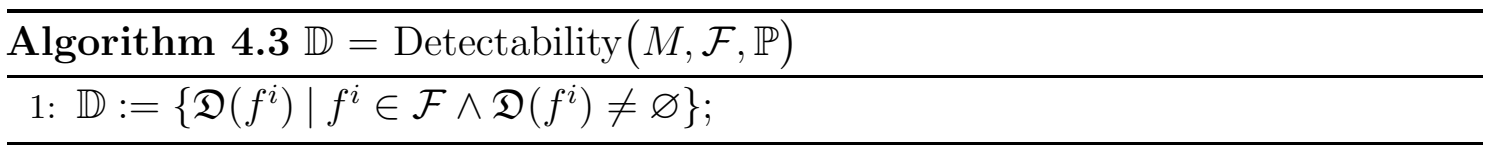

This theorem implies which sensor set can satisfy the maximum detectability requirement. Accordingly, there are a lot of sensor sets that can achieve the maximum detectability. However, this study takes an interest in a minimum sensor set since its cardinality is least. The minimum sensor set can be obtained from the family of detectability sets $\mathbb{D}$ using a minimal hitting set algorithm [91] or a greedy algorithm [77,123]. 
Example 4.1. Consider again the circuit example and the corresponding DCBG in Section 4.2. The linear DAEs model pertaining to the DCBG, without any additional sensor added, consists of 20 power variables and 5 sensor variables (i.e., 25 unknown variables in total) where none of six faults is detectable. Following Lemma 4.1, solving equations $H(s) Y^{i}(s)=F_{i}(s)$ yield solutions $Y^{i}(s)$. Then, the last five elements of these solutions are of the form

$$
\begin{aligned}
& {\left[Y^{V}(s), Y^{r}(s), Y^{C}(s), Y^{R_{1}}(s), Y^{R_{2}}(s), Y^{L}(s)\right]_{21 \rightarrow 25 \text { rows }}} \\
& =\left[\begin{array}{llllll}
* & * & * & * & * & * \\
* & * & * & * & * & * \\
* & * & * & * & * & * \\
* & * & * & * & * & * \\
* & * & * & * & * & *
\end{array}\right] \begin{array}{l}
y_{1} \\
y_{2} \\
y_{3} \\
y_{4} \\
y_{5}
\end{array} \\
& f^{V} \quad f^{r} \quad f^{C} \quad f^{R_{1}} \quad f^{R_{2}} \quad f^{L}
\end{aligned}
$$

Where each * in a row indicates a non-zero element and corresponds to a sensor variable $y_{k}$. Following (4.14), given the subset $\mathcal{P}_{i}$ with respect to each fault $f^{i}$ in $\mathcal{F}_{\text {e.g. }}$, the detectability set of fault $f^{i}$ can be identified by intersecting $\mathcal{P}_{i}$ with non-zero elements in the corresponding column of matrix (4.15). Thus, the detectability sets of this example are

$$
\begin{aligned}
& \mathfrak{D}\left(f^{V}\right)=\mathfrak{D}\left(f^{r}\right)=\left\{y_{1}, y_{2}, y_{3}\right\} \\
& \mathfrak{D}\left(f^{C}\right)=\left\{y_{1}, y_{2}\right\} \\
& \mathfrak{D}\left(f^{R_{1}}\right)=\left\{y_{1}, y_{2}, y_{3}, y_{4}, y_{5}\right\} \\
& \mathfrak{D}\left(f^{R_{2}}\right)=\left\{y_{4}, y_{5}\right\} \\
& \mathfrak{D}\left(f^{L}\right)=\left\{y_{3}, y_{4}, y_{5}\right\}
\end{aligned}
$$

Theorem 4.3 indicates that fault detectability is achieved since six detectability sets are nonempty and the minimum sensor sets have a non-empty intersection 
Chapter 4. Sensor Placement for Intrinsic Fault Isolability on the Bond Graph 99

with all of them. A greedy algorithm (or a minimal hitting set algorithm) is then applied to compute the minimum sensor sets of this example and thus they are $\left\{y_{1}, y_{4}\right\},\left\{y_{1}, y_{5}\right\},\left\{y_{2}, y_{4}\right\},\left\{y_{2}, y_{5}\right\}$.

\subsubsection{Sensor Placement for Isolability}

The previous subsection shows how to find minimum sensor sets that make those non-detectable faults in $\mathcal{F}$ detectable. Under the assumption that all faults are detectable, this subsection provides insight into obtaining minimum sensor sets for the achievement of maximum isolability.

The problem of achieving maximum isolability on the fault set $\mathcal{F}$ can be separated into $|\mathcal{F}|$ subproblems, one for each fault in $\mathcal{F}$. Each subproblem (which is related to fault $f^{i}$ ) is to find a set of sensors $\mathcal{S}$ so that a maximum number of faults are distinguishable from fault $f^{i}$. The solution of the isolability problem is then gained by combining the results of all subproblems. The following example is taken to illustrate the main principle.

Example 4.2. In Section 4.5.1, one can see that $\left\{y_{1}, y_{5}\right\}$ is one of the minimum sensor sets that can achieve detectability of all faults in Example 4.1. Thus, an extended model where all faults are detectable can be built by adding the equations $y_{1}=z_{1}$ and $y_{5}=z_{2}$. Additionally, in this extended model, there are eleven pairs of faults, i.e., $\left(f^{V}, f^{R_{1}}\right),\left(f^{V}, f^{R_{2}}\right),\left(f^{V}, f^{L}\right),\left(f^{r}, f^{R_{1}}\right),\left(f^{r}, f^{R_{2}}\right),\left(f^{r}, f^{L}\right),\left(f^{C}, f^{R_{1}}\right)$,$\left(f^{C}, f^{R_{2}}\right),\left(f^{C}, f^{L}\right),\left(f^{R_{1}}, f^{R_{2}}\right)$ and $\left(f^{R_{1}}, f^{L}\right)$, every pair of which are distinguishable from each other. This example will illustrate a procedure of achieving fault isolability in this extended model.

As analyzed above, the isolability procedure of this example can be divided into six steps, one for each subproblem. The intention of each step is to make as many faults as possible distinguishable from a specified fault. Consequently, the maximum isolability can be achieved in a step-by-step manner. 
The symmetry in distinguishability simplifies the procedure since it is unnecessary to compute detectability sets for pairs of faults already treated in the previously handled steps. The following exhibits the specific steps of this example. The first step is to distinguish $f^{r}, f^{C}, f^{R_{1}}, f^{R_{2}}, f^{L}$ from $f^{V}$. Because the distinguishability of $f^{V}$ from $f^{r}$ is already treated, the second step is to distinguish $f^{C}, f^{R_{1}}, f^{R_{2}}, f^{L}$ from $f^{r}$. The third step is to distinguish $f^{R_{1}}, f^{R_{2}}, f^{L}$ from $f^{C}$. The fourth step is to distinguish $f^{R_{2}}, f^{L}$ from $f^{R_{1}}$. The fifth step is to distinguish $f^{L}$ from $f^{R_{2}}$. The detectability sets in each step are gathered to compute the minimum sensor sets.

Consider the first step whose aim is to find sensors that achieve a maximumnumber-of-faults distinguishability from fault $f^{V}$. It follows from Theorem 4.2 that this is done by accomplishing detectability of a maximum number of faults when matrix $H(s)$ is redefined by $\left[H(s) F_{1}(s)\right]$. To avoid notational mix-up with $H(s)$ in the model of Example 4.1, the redefined matrix in the first step denoted by $H_{1}(s)$ is of the form

$$
H_{1}(s)=\left[\begin{array}{cc}
H(s) & F_{1}(s) \\
\Theta_{\mathrm{sb}}\left(y_{1}\right) & 0 \\
\Theta_{\mathrm{sb}}\left(y_{5}\right) & 0
\end{array}\right]
$$

where $F_{1}(s)$ is same as that in Example 4.1. Using Algorithm 4.3, the detectability sets for faults $f^{r}, f^{C}, f^{R_{1}}, f^{R_{2}}, f^{L}$ are

$$
\begin{aligned}
& \mathfrak{D}\left(f^{r}\right)=\varnothing, \mathfrak{D}\left(f^{C}\right)=\left\{y_{2}, y_{3}\right\}, \\
& \mathfrak{D}\left(f^{R_{1}}\right)=\mathfrak{D}\left(f^{R_{2}}\right)=\mathfrak{D}\left(f^{L}\right)=\varnothing .
\end{aligned}
$$

The sets $\mathfrak{D}\left(f^{R_{1}}\right), \mathfrak{D}\left(f^{R_{2}}\right)$ and $\mathfrak{D}\left(f^{L}\right)$ are empty because faults $f^{R_{1}}, f^{R_{2}}, f^{L}$ are already distinguishable from $f^{V}$ in the extended model. Conversely, the set $\mathfrak{D}\left(f^{r}\right)$ is empty because no additional sensor can make $f^{r}$ distinguishable from $f^{V}$. Why are both faults not distinguishable? $f^{V}$ and $f^{r}$ model faults occurred in the source of the circuit example in Figure 4.1 and they are influenced in the same way. 
Chapter 4. Sensor Placement for Intrinsic Fault Isolability on the Bond Graph101

In the second step, where $F_{2}(s)$ is merged with $H(s)$, the detectability sets for faults in $\left\{f^{C}, f^{R_{1}}, f^{R_{2}}, f^{L}\right\}$ are enumerated as

$$
\mathfrak{D}\left(f^{C}\right)=\left\{y_{2}, y_{3}\right\}, \mathfrak{D}\left(f^{R_{1}}\right)=\mathfrak{D}\left(f^{R_{2}}\right)=\mathfrak{D}\left(f^{L}\right)=\varnothing .
$$

The sets $\mathfrak{D}\left(f^{R_{1}}\right), \mathfrak{D}\left(f^{R_{2}}\right)$ and $\mathfrak{D}\left(f^{L}\right)$ are empty because pairs $\left(f^{r}, f^{R_{1}}\right),\left(f^{r}, f^{R_{2}}\right)$ and $\left(f^{r}, f^{L}\right)$ are distinguishable, respectively. In the third and fourth steps, all pairs of faults are already distinguishable and thus the respective detectability sets for these pairs of faults are empty. The fifth step only computes the detectability set for fault $f^{L}$. Algorithm 4.3 returns $\mathfrak{D}\left(f^{L}\right)=\left\{y_{3}, y_{4}\right\}$.

Since pairs of faults for the final step have been considered in the above five steps, the resulting sets are gathered to compute the sensor sets for the isolability procedure. Applying a greedy algorithm on the family of all non-empty detectability sets from all steps, gives rise to the minimal solutions $\left\{y_{3}\right\}$ and $\left\{y_{2}, y_{4}\right\}$. The subsequent context provides a formalization of the above procedure. For ease of presentation, a new symbol $M\left(f^{i}\right)$ must be introduced to denote a model that is obtained by decoupling fault $f^{i}$, i.e., column $F_{i}(s)$ is removed from $F(s)$ and merged with $H(s)$ as is done in (4.16).

Theorem 4.4. Assume that all faults in $\mathcal{F}$ are detectable in the model $M$. Let $\mathbb{P}$ be the family of all subsets $\mathcal{P}_{i}^{\prime}$ s and $M_{S}$ the equations pertaining to adding all sensors in a set $\mathcal{S}$. For a fault $f^{i}$, its maximum isolability means that a maximum number of faults in $\mathcal{F} \backslash\left\{f^{i}\right\}$ are distinguishable from it. Then the maximum isolability of a fault $f^{i}$ is achieved iff to each nonempty set in the family $\mathbb{D}_{i}=$ $\operatorname{Detectability}\left(M\left(f^{i}\right), \mathcal{F} \backslash\left\{f^{i}\right\}, \mathbb{P}\right)$, its intersection with $\mathcal{S}$ is nonempty.

Proof. Assume that $\mathfrak{D}\left(f^{j}\right) \in \mathbb{D}_{i}\left(f^{j} \in \mathcal{F} \backslash\left\{f^{i}\right\}\right)$ and $\mathcal{S} \cap \mathfrak{D}\left(f^{j}\right)=\varnothing$. This means that fault $f^{j}$ is not distinguishable from fault $f^{i}$. However, since $\mathfrak{D}\left(f^{j}\right) \neq \varnothing, \mathcal{S}$ can be extended in order that $\mathfrak{D}\left(f^{j}\right) \cap \mathcal{S} \neq \varnothing$. Therefore, maximum isolability 


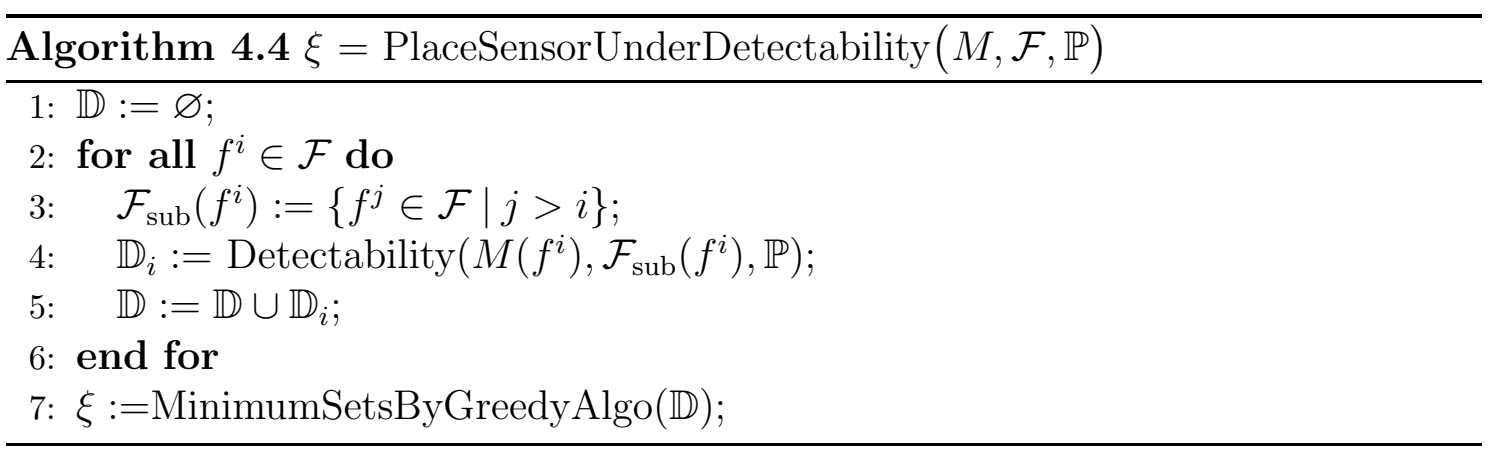

of fault $f^{i}$ implies that $\mathcal{S}$ intersecting with all nonempty sets in $\mathbb{D}_{i}$ is nonempty. Alternatively, if $\mathcal{S}$ intersecting with all sets in $\mathbb{D}_{i}$ is nonempty, then according to Theorem 4.3, a maximum number of faults are detectable in $M\left(f^{i}\right) \cup M_{S}$. In other words, a maximum number of faults are distinguishable from $f^{i}$ in $M \cup M_{S}$.

The above theorem shows the necessary and sufficient condition for maximum isolability of fault $f^{i}$ from the remaining faults in $\mathcal{F} \backslash\left\{f^{i}\right\}$. The following corollary discusses how to attain maximum isolability of a fault set.

Corollary 4.1. For a set of faults $\mathcal{F}$, its maximum isolability is attained iff to each nonempty set in the family $\mathbb{D}=\underset{f^{i} \in \mathcal{F}}{\cup} \mathbb{D}_{i}$, its intersection with $\mathcal{S}$ is nonempty.

Proof. Maximum isolability of a fault set in the model $M \cup M_{S}$ is achieved if each fault in $\mathcal{F}$ fulfills maximum isolability. Conversely, if maximum isolability of a fault set is attainable, each fault $f^{i}$ in $\mathcal{F}$ must achieve maximum isolability. Therefore, it follows from Theorem 4.4 that this corollary holds straightforwardly.

The condition of maximum isolability in Corollary 4.1 can be translated into an algorithm that, given a DAE model $M$, a set of detectable faults $\mathcal{F}$ and a family $\mathbb{P}$ of subsets $\mathcal{P}_{i}{ }^{\prime} s$, returns a family of minimum sensor sets. The associated pseudo-code is shown in Algorithm 4.4 .

Recall that the above discussion is based on the assumption that all faults in $\mathcal{F}$ are detectable. The next subsection considers the sensor placement problem for fault detectability and isolability with this assumption removed. 
Chapter 4. Sensor Placement for Intrinsic Fault Isolability on the Bond Graph103

\subsubsection{Sensor Placement for Both Detectability and Isolabil- ity}

Section 4.5.1 presents how to place sensors under the accomplishment of detectability. The previous subsection gives a discussion on maximum isolability in the model where all faults are assumed detectable. This subsection focuses on analyzing the sensor placement problem for both detectability and isolability and provides a synthesized algorithm that arises from combining the algorithms in two aforementioned subsections.

Example 4.3. Consider Example 4.1 again. Section 4.5.1 shows that the minimum sensor sets that accomplish detectability on the fault set $\mathcal{F}_{\text {e.g. }}$ are

$$
\left\{y_{1}, y_{4}\right\},\left\{y_{1}, y_{5}\right\},\left\{y_{2}, y_{4}\right\},\left\{y_{2}, y_{5}\right\}
$$

In Section 4.5.2, $\left\{y_{1}, y_{5}\right\}$ is chosen and two sensors measuring $y_{1}, y_{5}$ are added in the model. As an example, this extended model gives rise to the following minimum sensor sets that achieve maximum isolability on the fault set $\mathcal{F}_{\text {e.g. }}$ :

$$
\left\{y_{3}\right\},\left\{y_{2}, y_{4}\right\}
$$

Note that the achievement of maximum isolability is based on the addition of two sensors measuring $y_{1}, y_{5}$. The resulting sets are

$$
\left\{y_{1}, y_{3}, y_{5}\right\},\left\{y_{1}, y_{2}, y_{4}, y_{5}\right\}
$$

which fulfill both detectability and maximum isolability requirements. In addition to $\left\{y_{1}, y_{5}\right\}$, (4.17) has three other sets that achieve detectability. The same procedures as for $\left\{y_{1}, y_{5}\right\}$ are thus taken for these three sets to obtain the minimum sensor sets. 


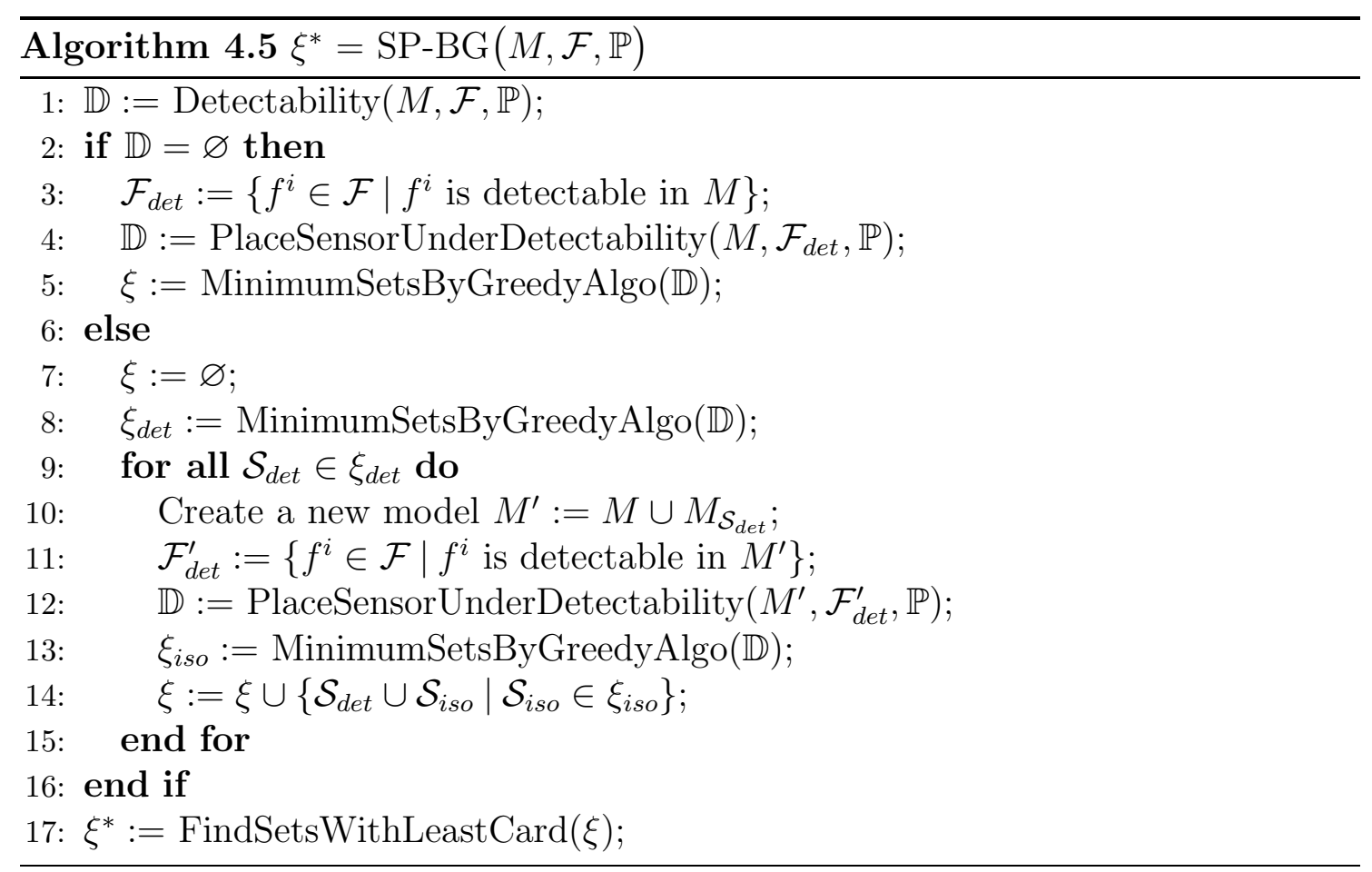

Among the resultant sensor sets, $\left\{y_{1}, y_{3}, y_{5}\right\}$ is a minimum sensor set with the least cardinality that fulfills the requirements of detectability and maximum isolability.

Recall that a fault that is not detectable, cannot be made isolable from the other faults. Additionally, all detectable faults are distinguishable from the non-detectable ones. Therefore, a sufficiency of accomplishing maximum isolability is to achieve firstly maximum detectability and then maximum isolability amongst all detectable faults. Following this spirit, the algorithm below, given a model $M$, the set of faults $\mathcal{F}$ and the family $\mathbb{P}$ of all subsets $\mathcal{P}_{i}{ }^{\prime} s$ with respect to faults in $\mathcal{F}$, is developed to compute all minimum sensor sets with the least cardinality. Let $\xi^{*}$ denote the set of all solutions computed by Algorithm 4.5.

\subsection{Case Studies}

This section demonstrates the proposed sensor placement method for two case study examples: (a) the circuit example with an alternative causal path configura- 
Chapter 4. Sensor Placement for Intrinsic Fault Isolability on the Bond Graph105

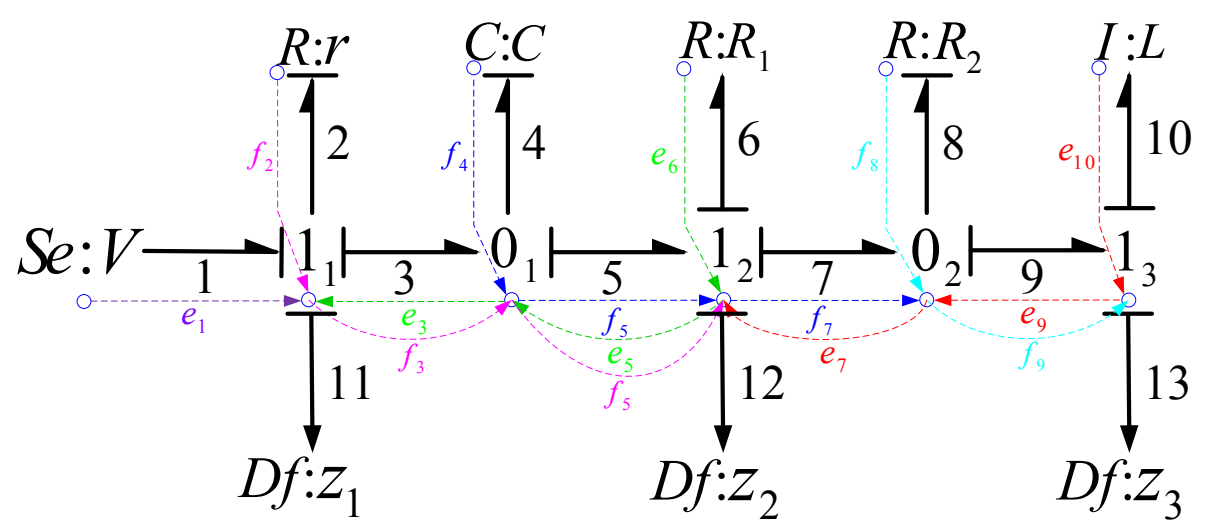

Figure 4.7: The alternative DCBG with six causal paths and three sensors

tion and (b) a mechatronic system.

\subsubsection{An Circuit Example with the Alternative Casual Path Configuration}

On a BG, there is some flexibility in constructing the ACDAL of a storage element with one of its adjacent elements. The circuit example in Figure 4.1 has an alternative DCBG. The alternative DCBG is used to illustrate the proposed algorithm for sensor placement. Different with the DCBG in Figure 4.2, the alternative DCBG pulls the compliance element $C$ and the resistance element $R_{1}$ together to form an ACDAL. Figure 4.7 shows the corresponding DCBG. On this DCBG, assignment rules of causal paths are applied to set causal paths with respect to each fault in $\mathcal{F}_{\text {e.g. }}$. As a result, six causal paths pertaining to faults in $\mathcal{F}_{\text {e.g. }}$ are shown in Figure 4.7 and the respective subsets of junctions are $\mathcal{J}_{V}=\left\{\mathbf{1}_{1}\right\}, \mathcal{J}_{r}=\left\{\mathbf{1}_{1}, \mathbf{0}_{1}, \mathbf{1}_{2}\right\}, \mathcal{J}_{C}=$ $\left\{\mathbf{0}_{1}, \mathbf{1}_{2}, \mathbf{0}_{2}\right\}, \mathcal{J}_{R_{1}}=\left\{\mathbf{1}_{1}, \mathbf{0}_{1}, \mathbf{1}_{2}\right\}, \mathcal{J}_{R_{2}}=\left\{\mathbf{0}_{2}, \mathbf{1}_{3}\right\}$ and $\mathcal{J}_{L}=\left\{\mathbf{1}_{2}, \mathbf{0}_{2}, \mathbf{1}_{3}\right\}$. After substitution junction $\mathbf{1}$ (or $\mathbf{0}$ ) with sensor variable $y_{k}$, the resulting subsets of sensor variables are listed as $\mathcal{P}_{V}=\left\{y_{1}\right\}, \mathcal{P}_{r}=\left\{y_{1}, y_{2}, y_{3}\right\}, \mathcal{P}_{C}=\left\{y_{2}, y_{3}, y_{4}\right\}, \mathcal{P}_{R_{1}}=$ $\left\{y_{1}, y_{2}, y_{3}\right\}, \mathcal{P}_{R_{2}}=\left\{y_{4}, y_{5}\right\}$ and $\mathcal{P}_{L}=\left\{y_{3}, y_{4}, y_{5}\right\}$. Given the fault set $\mathcal{F}_{\text {e.g. }}$ and the set $\mathbb{P}=\left\{\mathcal{P}_{V}, \mathcal{P}_{r}, \mathcal{P}_{C}, \mathcal{P}_{R_{1}}, \mathcal{P}_{R_{2}}, \mathcal{P}_{L}\right\}$, Algorithm 4.5 is invoked to give the optimal solution $\left\{y_{1}, y_{3}, y_{5}\right\}$ that achieves maximum isolability (i.e., each pair in $\left\{\left(f^{i}, f^{j}\right) \in\right.$ 
$\left.\mathcal{F}_{\text {e.g. }} \times \mathcal{F}_{\text {e.g. }} \mid\left(f^{i}, f^{j}\right) \neq\left(f^{V}, f^{r}\right)\right\}$ are distinguishable from each other $)$. One already sees that two different causality configurations of the circuit example have the same result. This is not surprising because this chapter solves the same sensor placement issue of the circuit example and also an indication that the sensor placement approach performs well.

Although two optimized results under different causality configurations are identical, readers may arise a question whether the set $\left\{y_{1}, y_{3}, y_{5}\right\}$ really accomplishes maximum isolability or not. Therefore, primary ARRs are derived to answer this question. After three sensors $\left\{z_{1}, z_{2}, z_{3}\right\}$ measuring $\left\{y_{1}, y_{3}, y_{5}\right\}$ are installed, three primary ARRs arise from the constitutive relations of $\mathrm{BG}$ elements $\left\{r, \mathbf{0}_{1}, \mathbf{0}_{2}\right\}$ in Figure 4.7.

For the BG element $r$, the constitutive relation of it is

$$
e_{2}=r f_{2}
$$

where $f_{2}$ is measurable by $z_{1}$, hence $f_{2}=z_{1}$; but $e_{2}$ is an unknown variable. The constitutive relation on junction $\mathbf{1}_{1}$ shows that $e_{2}=e_{1}-e_{3}=V-e_{3}$. By covering the casual path, $e_{3}=e_{5}=e_{6}+e_{7}$. Since $e_{6}=R_{1} f_{6}$ and $f_{6}=z_{2}, e_{6}=R_{1} z_{2}$. As for $e_{7}$, the causal path indicates that $e_{7}=e_{9}=e_{10}=L \dot{f}_{10}$ and $f_{10}=z_{3}$. These solutions lead (4.18) to the first ARR equation

$$
r_{8}=V-L \dot{z}_{3}-R_{1} z_{2}-r z_{1}
$$

For junction $\mathbf{0}_{1}$, its constitutive relation is

$$
f_{3}-f_{4}-f_{5}=0
$$

where $f_{3}$ and $f_{5}$ are measurable by sensors $z_{1}$ and $z_{2}$, respectively; and $f_{4}$ is an 
Chapter 4. Sensor Placement for Intrinsic Fault Isolability on the Bond Graph107

Table 4.1: The FSM of the circuit example.

\begin{tabular}{c|cccccc} 
& $f^{V}$ & $f^{r}$ & $f^{C}$ & $f^{R_{1}}$ & $f^{R_{2}}$ & $f^{L}$ \\
\hline$r_{8}$ & 1 & 1 & 0 & 1 & 0 & 1 \\
$r_{9}$ & 0 & 0 & 1 & 1 & 0 & 1 \\
$r_{10}$ & 0 & 0 & 0 & 0 & 1 & 1
\end{tabular}

unknown variable. The constitutive relation of $C$ shows that $f_{4}=C \dot{e}_{4}$ where $e_{4}=e_{5}$. The derivation of the previous $\mathrm{ARR}$ shows that $e_{5}=R_{1} z_{2}+L \dot{z}_{3}$. These solutions lead (4.20) to the second ARR equation

$$
r_{9}=z_{1}-C\left(R_{1} \dot{z}_{2}+L \ddot{z}_{3}\right)-z_{2}
$$

Next, the constitutive relation of junction $\mathbf{0}_{2}$ is

$$
f_{7}-f_{8}-f_{9}=0
$$

where $f_{7}$ and $f_{9}$ are measurable by $z_{2}$ and $z_{3}$, respectively; and $f_{8}$ is an unknown variable. The constitutive relation of $R_{2}$ indicates that $f_{8}=e_{8} / R_{2}$ where $e_{8}=e_{9}=$ $e_{10}=L \dot{z}_{3}$. Therefore, by substituting these solutions into (4.22), the third ARR is

$$
r_{10}=z_{2}-\frac{L}{R_{2}} \dot{z}_{3}-z_{3}
$$

In summary, $r_{8}, r_{9}, r_{10}$ are three primary ARRs since anyone of them cannot be expressed in terms of the rest. Tabulating the fault information of these three ARRs yields a fault signature matrix (FSM) given in Table 4.1. The columns of FSM imply that each fault in $\left\{f^{C}, f^{R_{1}}, f^{R_{2}}, f^{L}\right\}$ is isolable from the remaining faults in $\mathcal{F}_{\text {e.g. }}$ and faults $f^{V}, f^{r}$ are distinguishable from faults in $\mathcal{F}_{\text {e.g. }} \backslash\left\{\left(f^{V}, f^{r}\right)\right\}$ whereas they are not distinguishable from each other. This is because faults $f^{V}, f^{r}$ originate from the voltage source and are influenced by the same way. The analysis on FSM brings a conclusion that maximum isolability is achieved when the circuit example 
is equipped with these three sensors. The above discussion from a set of ARRs viewpoint validates that $\left\{y_{1}, y_{3}, y_{5}\right\}$ is the optimal set for maximum isolability.

\subsubsection{A Mechatronic System}

The main goal of this subsection is to illustrate the sensor placement problem on another type of ACDAL structures that are connected by a gyrator. Therefore, a mechatronic system is employed to make a demonstration on such an ACDAL.

Figure 4.8 depicts the schematic diagram of a DC motor whose input is interfaced with a voltage divider circuit. This circuit is a mechatronic system whose objective is to rotate a mechanical load that is attached to the motor shaft. The angular velocity of the motor shaft is adjusted by the mechanism of the voltage divider. The voltage divider consists of components $\left\{V_{i n}, R_{1}, R_{2}\right\}$. $V_{\text {in }}$ represents a constant DC voltage source with $V_{i n}$ volt. $R_{1}$ is a constant resistor with a resistance value $R_{1}$ ohm, where $R_{2}$ is a rheostat with a resistance value $R_{2}$ ohm. $R_{1}$ and $R_{2}$ form the voltage divider circuit that changes the input voltage of the DC motor that is denoted by $e_{i n}$. The change of input voltage is achieved by varying the resistance value of $R_{2}$. The DC motor consists of an inductor $L_{1}$ and a mechanical load attached to the motor shaft. $L_{1}$ represents an ideal inductor (i.e., which has no resistance) with an inductance value of $L_{1}$ Henries; $\omega$ denotes the angular velocity of the motor shaft; and $L_{2}$ represents the inertia of the motor shaft with the load attached. The rotational friction across the motor shaft is assumed to be zero. Figure 4.9 describes the DCBG of this mechatronic system.

Note that this DCBG has four junctions $\left\{\mathbf{1}_{1}, \mathbf{0}_{1}, \mathbf{1}_{2}, \mathbf{1}_{3}\right\}$. Each junction is assigned to a sensor variable $y_{k}$ by sequence and thus a set of sensor variables $\mathcal{P}=$ $\left\{y_{1}, y_{2}, y_{3}, y_{4}\right\}$ results. The mechatronic system considers three faults from 1-port elements $\left\{V_{i n}, L_{1}, L_{2}\right\}$. Thus, the fault set of this system is $\mathcal{F}_{\text {mech }}=\left\{f^{V_{i n}}, f^{L_{1}}, f^{L_{2}}\right\}$. Two assignment rules for causal paths are applied on this DCBG and the resulting 
Chapter 4. Sensor Placement for Intrinsic Fault Isolability on the Bond Graph109

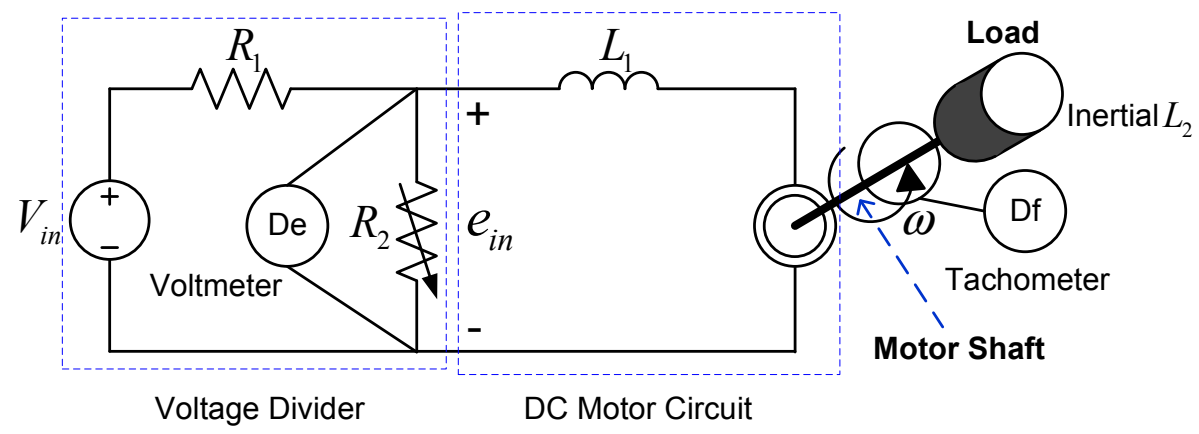

Figure 4.8: The mechatronic system: A DC-motor interfaced with voltage divider.

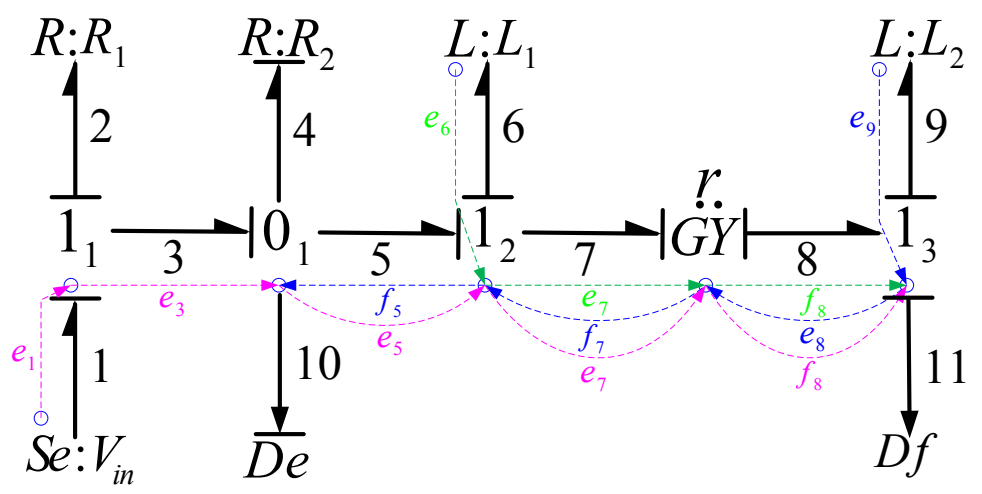

Figure 4.9: The DCBG of mechatronic system with three causal paths and two sensors.

causal paths are shown in Figure 4.9. Extracting junctions on causal paths with respect to faults in $\mathcal{F}$ yields the respective subsets of junctions $\mathcal{J}_{V_{\text {in }}}=\left\{\mathbf{1}_{1}, \mathbf{0}_{1}, \mathbf{1}_{2}, \mathbf{1}_{3}\right\}$, $\mathcal{J}_{L_{1}}=\left\{\mathbf{1}_{2}, \mathbf{1}_{3}\right\}$ and $\mathcal{J}_{L_{2}}=\left\{\mathbf{0}_{1}, \mathbf{1}_{2}, \mathbf{1}_{3}\right\}$. Substituting junctions with sensor variables $y_{k}{ }^{\prime} s$ one by one, the sets of sensor variables are $\mathcal{P}_{V_{i n}}=\left\{y_{1}, y_{2}, y_{3}, y_{4}\right\}, \mathcal{P}_{L_{1}}=\left\{y_{3}, y_{4}\right\}$ and $\mathcal{P}_{L_{2}}=\left\{y_{2}, y_{3}, y_{4}\right\}$. Execution of Algorithm 4.5, if the set $\mathbb{P}=\left\{\mathcal{P}_{V_{i n}}, \mathcal{P}_{L_{1}}, \mathcal{P}_{L_{2}}\right\}$ and the fault set $\mathcal{F}=\left\{f^{V_{i n}}, f^{L_{1}}, f^{L_{2}}\right\}$ are given, produces the minimum sensor set $\left\{y_{2}, y_{4}\right\}$ with least cardinality. These two sensors measuring $y_{2}, y_{4}$ are a voltmeter $D e$ and a tachometer $D f$ shown in Figure 4.8. One measures $e_{i n}$ and the other is attached to the motor shaft, measuring the angular velocity of the shaft. With these two sensors installed, two primary ARRs are derived from constitutive relations of BG elements $\left\{\mathbf{1}_{1}, G Y\right\}$, respectively. As a result, these two ARRs are of the 
Table 4.2: The FSM of the mechatronic system

\begin{tabular}{c|ccc} 
& $f^{V_{i n}}$ & $f^{L_{1}}$ & $f^{L_{2}}$ \\
\hline$r_{11}$ & 1 & 0 & 1 \\
$r_{12}$ & 0 & 1 & 1
\end{tabular}

following form

$$
\begin{aligned}
& r_{11}=V_{i n}-D e-R_{1}\left\{\frac{D e}{R_{2}}+\frac{L_{2}}{r} \dot{D} f\right\} \\
& r_{12}=D f-\frac{1}{r}\left\{D e-\frac{L_{1} L_{2}}{r} \ddot{D} f\right\},
\end{aligned}
$$

where $r$ is the gyrator modulus. The relationship between faults in $\left\{f^{V_{i n}}, f^{L_{1}}, f^{L_{2}}\right\}$ and ARRs $\left\{r_{11}, r_{12}\right\}$ can be depicted by a FSM in Table 4.2. The column analysis on this FSM indicates that each fault is different from the rest. This implies that each fault in $\left\{f^{V_{i n}}, f^{L_{1}}, f^{L_{2}}\right\}$ is isolable from the others. This case study also verifies the excellent performance of this sensor placement approach.

\subsection{Summary}

This chapter studies the sensor placement issue on a BG where the behaviors of the elements are linear and then proposes the SP-BG for sensor placement. On the BG, junctions are regarded as possible sensor locations and causal paths build a bridge between faults and junctions. The analysis on causal paths from faults to junctions reveals what subset of junctions can make a fault diagnosable. In the DAEs model regarding to a DCBG, two basic properties of sensor placement, i.e., detectability and distinguishability, are investigated under the junction restrictions of each fault. Both basic properties are analyzed in the image space of the DAEs model rather than a set of ARRs, which is a distinct feature of this new approach. Based on detectability and isolability analysis, the SP-BG is devised to compute minimum sensor sets that achieve maximum isolability. The optimal set of junctions produced by the SP-BG is validated by deriving primary ARRs on the corresponding 
Chapter 4. Sensor Placement for Intrinsic Fault Isolability on the Bond Graph111

DCBG. Two representative examples illustrate the effectiveness of this approach.

Compared with the SPLCDP in Chapter 3, the SP-BG circumvents generating a lot of useless ARRs and somewhat improves its performance. Simultaneously, the SP-BG only deals with the linear and dynamic models for sensor placement, which is the biggest disadvantage. 


\section{Chapter 5}

\section{An Efficient Method for Placing Sensor on the Bond Graph}

This chapter further exploits the characteristic of BGs and develops a more efficient approach for sensor placement than the SP-BG in Chapter 4 in the context of BG modelling. The approach benefits from the necessary and sufficient conditions for detectability which are derived on the basis of a conclusion given in [89,121]. This approach consists of three parts. The first part uses causal paths to analyze what subset of junctions contributes to fault detectability and isolability. The second part offers a simpler detectability condition and a simpler necessary condition for distinguishability of two faults in a DAEs model. On gathering consequences from this simpler distinguishability condition, an optimization problem is formulated as searching the optimal set of junctions for maximum isolability on the fault set $\mathcal{F}$. Thus, the final part develops a DP algorithm to solve this optimization problem.

This chapter is organized as follows. Section 5.1 briefly summarizes the fault set and the set of junctions considered for sensor placement; it re-exhibits two assignment rules of causal paths for a fault and then uses them to yield the subset of junctions for each fault; it moreover restates a linear DAEs model regarding to a 
DCBG which has been established in Chapter 4. Section 5.2 exploits the detectability condition and the distinguishability condition and then formulates an optimization problem for fault isolability. Section 5.3 develops a DP algorithm to solve the formulated optimization problem for obtaining the optimal solution. Section 5.4 studies the two-tank system to demonstrate this approach for sensor placement and validates the corresponding results by deriving specific primary ARRs and uses two other case studies to compare with the SP-BG in terms of the performance. Finally, Section 5.5 provides the summary.

\subsection{A Brief Summary of Placing Sensors on the Bond Graph}

This chapter continues to discuss the sensor placement problem in a linear dynamic system. The BG modelling technique is a way of establishing the graphical model for a linear dynamic system. In this chapter, All 1-port BG elements are assumed to behave linearly. These constitutive relations are summarized in Table 2.2. This chapter continues to focus on placing sensors on the DCBG. Definition 4.1 is related to the notion of the DCBG.

Similar to Chapter 4, this chapter also pays particular attention to causal paths that provide causal connections between 1-port BG elements and junctions. Then, these causal paths are used to systematically eliminate unknown system's variables and thus to form primary ARRs. Moreover, this chapter concerns all possible faults occurred in 1-port elements. Let $\mathcal{F}=\left\{f^{1}, f^{2}, \ldots, f^{Q}\right\}$ denote the set of faults. Junctions are normally regarded as all possible sensor locations in a BG. For notational convenience, let $\mathcal{J}=\left\{\mathbf{0}_{1}, \mathbf{0}_{2}, \ldots, \mathbf{0}_{K_{1}}, \mathbf{1}_{1}, \mathbf{1}_{2}, \ldots, \mathbf{1}_{K_{2}}\right\}$ denote the set of $K$ junctions where $K_{1}+K_{2}=K$. The next subsection utilizes two assignment rules of causal paths to determine what subset of junctions contributes to fault detectability and 
isolability.

\subsubsection{A Brief Statement of Two Assignment Rules}

An algebraic loop arises from constitutive relations of a closed causal path between two 1-port elements. Among algebraic loops, the ACDAL (see Definition 4.3) leads to a non-homogeneous ODE which makes the associated power variables to have multiple values. The multi-valued power variables make trouble in tracing back the cause of a fault. A causal path starts from a faulty 1-port element and ends at a junction; for sensor placement, its length is restricted to prevent multi-valued power variables being present. Two assignment rules of causal paths regarding to faults have been introduced in Chapter 4. Hence, there are two groups of faults which are separated by the ACDAL. One group of faults occurs in 1-port elements of the ACDAL, whilst the other group are those not involved in any the ACDAL element. Each assignment rule is made to determine the length of causal paths for faults in one group. For convenience, these two assignment rules are restated here.

Rule 5.1 (same as Rule 4.1). For a fault occurring in an ACDAL element, its causal path passes through two junctions of this ACDAL and then extends to one adjacent junction of this $A C D A L$ if possible.

Rule 5.2 (same as Rule 4.2). For any fault $f^{i}$ not occurring in ACDALs, its causal path, if intersecting with less than two ACDALs, terminates at the last junction along this causal path; otherwise the causal path, if intersecting with two or more $A C D A L s$, terminates at the second junction of the second ACDAL.

Applying the above two assignment rules on the fault set $\mathcal{F}$ yields the causal paths for each fault $f^{i}$ in $\mathcal{F}$ which have been outlined on the DCBG. For ease of mathematical description in the following sections, it is necessary to convert the causal path regarding to a fault into a set of junctions. Counting junctions along 
the causal path with respect to fault $f^{i}$ results in the associated subset of junctions of $\mathcal{J}$ for fault detectability and isolability, noted by $\mathcal{J}_{i}$. Hence, the BG gives $Q$ subsets of junctions $\mathcal{J}_{1}, \mathcal{J}_{2}, \ldots, \mathcal{J}_{Q}$ corresponding to faults $f^{1}, f^{2}, \ldots, f^{Q}$.

\subsubsection{A Brief Statement of the Linear DAEs Model}

The linear DAEs model has been described in Section 4.4.1. This subsection just presents a brief summary of the linear DAEs model. The DAEs model

$$
H(s) x+L(s) z+F(s) f=\mathbf{0},
$$

where $x=\left[x_{1}^{\top}, y_{1}, y_{2}, \cdots, y_{K}\right]^{\top}$. The symbol $x_{1}$ indicates the vector of $2 n$ conjugate power variables appearing equation (2.26). $y_{k}(k=1,2, \ldots, K)$ is defined as a sensor variable in one-to-one correspondence to each junction in $\mathcal{J}$. $z$ is a vector of inputs and outputs of the BG. $f=\left[\Delta_{1}, \Delta_{2}, \ldots, \Delta_{Q}\right]^{\top}$ is a vector of $Q$ fault signals of concern in this chapter. $L(s)$ and $F(s)$ are two polynomial matrices defined in Section 4.1. According to fault description in Section $2.4, F(s)$ is actually an integer matrix which is composed of the elements 0 and -1. For $H(s)$ in (5.1), as noted in Section 4.1, there exists an elementary matrix $U(s)$ such that

$$
U(s) H(s)=\left[\begin{array}{cc}
B_{s}^{n}(s) & \mathbf{0} \\
\Gamma & I_{K} \\
\mathbf{0} & \mathbf{0}
\end{array}\right]
$$

where $B_{s}^{n}(s)$ is a square matrix associated with an $n$-bond graph model, $\Gamma$ denotes a matrix with size $2 n \times K$, and $I_{K}$ is a $K$-by- $K$ identity matrix. Obviously, matrix $H(s)$ has full column rank.

As shown in Section 4.1, all junctions in $\mathcal{J}$ have built one-to-one correspondence relations with sensor variables $y_{1}, y_{2}, \cdots, y_{K}$ in sequence. Sequentially substituting 
each member in $\mathcal{J}$ with a sensor variable $y_{k}$ yields a new set of sensor variables, symbolized by $\mathcal{P}$. Similar to the set $\mathcal{P}$, the subset $\mathcal{P}_{i}$ of $\mathcal{P}$ with respect to fault $f^{i}$ is directly produced by the replacement of all junctions in the set $\mathcal{J}_{i}$. Hence, the BG with the fault set $\mathcal{F}$ has $Q$ subsets of sensor variables $\mathcal{P}_{i}^{\prime} s, 1 \leq i \leq Q$.

\subsection{Problem Formulation for Sensor Placement}

In the DAEs model, this section shows a discussion on non-detectable faults centered around two conditions of fault detectability and distinguishability. For a nondetectable fault, this section provides a simpler necessary and sufficient condition for detectability. Meanwhile, this section offers a simpler necessary condition to distinguish two non-detectable faults. Finally, an optimization problem is formulated as finding the optimal set of junctions for fault isolability on the fault set $\mathcal{F}$.

\subsubsection{Necessary and Sufficient Conditions for Fault Detectabil- ity}

For ease of presentation in the necessary and sufficient condition for detectability of a non-detectable fault, the notion of a sensor binary vector $\Theta_{\mathrm{sb}}\left(y_{k}\right)$ defined in the previous chapter (see Definition 4.4) is still used in the following context. In a DAEs model, a condition for fault detectability presented in $[89,121]$ is expressed as follows.

Theorem 5.1 (Frisk et al. [89,121]). Fault $f^{i}$ is detectable in (5.1) iff $F_{i}(s) \notin$ $\operatorname{Im}[H(s)]$.

The above theorem can be performed by a rank test, i.e., $\operatorname{Rank}\left[H(s) F_{i}(s)\right]>$ $\operatorname{Rank}[H(s)]$. Intuitively, for any non-detectable fault $f^{i}$, it follows that $F_{i} \in \operatorname{Im}[H(s)]$. The subsequent context offers the discussion about which sensor variable in $\mathcal{P}$ measured by a sensor can make a non-detectable fault $f^{i}$ detectable. 
Lemma 5.1 (same as Lemma 4.1). Let $f^{i} \in \mathcal{F}$ be a non-detectable fault, $\mathcal{P}_{i} \subseteq \mathcal{P}$ the set of sensor variables with respect to fault $f^{i}$, and $Y(s)$ the unique solution to

$$
H(s) Y(s)=F_{i}(s)
$$

Then fault $f^{i}$ becomes detectable iff any unknown variable $y_{k}$ in the set $\left\{y_{k} \in \mathcal{P}_{i}\right.$ : $\left.Y_{2 n+k}(s) \neq 0\right\}$ is measured.

This lemma is identical to Lemma 4.1. For readability, it is stated again. Clearly, $\left\{y_{k} \in \mathcal{P}_{i}: Y_{2 n+k}(s) \neq 0\right\} \subseteq \mathcal{P}_{i}$. It follows from Lemma 5.1 that any sensor variable in $\mathcal{P}_{i}$ measured by a sensor is the necessary condition to make a non-detectable fault detectable. Determining the set $\left\{y_{k} \in \mathcal{P}_{i}: Y_{2 n+k}(s) \neq 0\right\}$ in the previous chapter needs two steps : (i) solving the equation (5.2) and (ii) judging whether the corresponding items $Y_{2 n+k}(s)^{\prime} s$ are non-zero. In order to avoid solving linear equations and evaluate detectability from the model property, a significant question arises out whether the set $\left\{y_{k} \in \mathcal{P}_{i}: Y_{2 n+k}(s) \neq 0\right\}$ is equal to $\mathcal{P}_{i}$. In order to answer this question, the following lemma is presented.

Lemma 5.2. For a non-detectable fault $f^{i}$, i.e., $F_{i}(s) \in \operatorname{Im}[H(s)]$, in the model (5.1), if any sensor variable $y_{k} \in \mathcal{P}$ is measured by a sensor, then $\left[\begin{array}{c}F_{i}(s) \\ 0\end{array}\right] \notin$ $\operatorname{Im}\left[\begin{array}{c}H(s) \\ \Theta_{\mathrm{sb}}\left(y_{k}\right)\end{array}\right]$

Proof. This lemma will be proved by contradiction. It is assumed that there exists a sensor variable $y_{k}$ such that if it is measured by a sensor, $\left[\begin{array}{c}F_{i}(s) \\ 0\end{array}\right] \in \operatorname{Im}\left[\begin{array}{c}H(s) \\ \Theta_{\mathrm{sb}}\left(y_{k}\right)\end{array}\right]$ still holds. Let $G(G=2 n+K)$ be the size of $x$ in $(5.1)$. Due to $\left[\begin{array}{c}F_{i}(s) \\ 0\end{array}\right] \in$ 
$\operatorname{Im}\left[\begin{array}{c}H(s) \\ \Theta_{\mathrm{sb}}\left(y_{k}\right)\end{array}\right]$, there must exist a vector $C=\left[c_{e_{1}}, c_{f_{1}}, \cdots, c_{f_{n}}, c_{y_{1}}, \cdots, c_{y_{K}}\right]^{\top}$, satisfying $\Theta_{\mathrm{sb}}\left(y_{k}\right) C=0$ and $H(s) C=F_{i}(s)$ simultaneously, i.e.,

$$
\begin{aligned}
& c_{y_{k}}=0 \\
& c_{e_{1}} H_{1}^{c}(s)+c_{f_{1}} H_{2}^{c}(s)+\cdots+c_{y_{K}} H_{G}^{c}(s)=F_{i}(s),
\end{aligned}
$$

where $H_{g}^{c}(s)$ denotes the $g$-th column of $H(s)$. Furthermore, note that there must exist an elementary matrix $U$ such that

$$
U H(s)=\left[\begin{array}{cc}
B_{s}^{n}(s) & \mathbf{0} \\
\Gamma & I_{K} \\
\mathbf{0} & \mathbf{0}
\end{array}\right] \text { and } U F_{i}(s)=\left[\begin{array}{c}
\mathbf{0}_{(2 n-2) \times 1} \\
-1 \\
0 \\
\mathbf{0}
\end{array}\right]
$$

where $B_{s}^{n}(s)$ is a square matrix related to a BG with $n$ bonds (i.e., $B_{s}^{n}(s)$ has full rank) and $I_{K}$ is an identity matrix with size $K \times K$. For ease of presentation, it is necessary to introduce two additional notations $F_{i}^{*}(s)=\left[\mathbf{0}_{1 \times(2 n-2)},-1,0\right]^{\top}$ with order $2 n \times 1$ and $C^{2 n}=\left[c_{e_{1}}, c_{f_{1}}, \cdots, c_{f_{n}}\right]^{\top}$ (which is a vector of the first $2 n$ entries in $C$ ). Then, (5.4) is equivalent to the following equations

$$
\begin{aligned}
& B_{s}^{n}(s) C^{2 n}=F_{i}^{*}(s) \\
& \Gamma C^{2 n}+\left[c_{y_{1}}, \ldots, c_{y_{K}}\right]^{\top}=\mathbf{0} .
\end{aligned}
$$

The discussion on proving this lemma will be divided into two cases. One case is that the sensor variable $y_{k}$ and fault $f^{i}$ relate to the same junction; the other is that the $y_{k}$ and fault $f^{i}$ pertain to two separate junctions.

Now discuss the first case. Without loss of generality, it is assumed that the 
$y_{k}$ relates to a $\mathbf{0}$-junction which has $l$ bonds and connects with an inertia $C$ by a bond numbered $q$. Simultaneously, fault $f^{i}$ is assumed to occur on the BG element $C$. According to fault modeling, the faulty constitutive relation is $f_{q}=C s e_{q}+\Delta_{i}$ (where $\Delta_{i}$ is closely related to the unique non-zero element in $F_{i}$ ). In this $\mathbf{0}$-junction, all effort variables $e_{q}, e_{q+1}, \ldots, e_{q+l-1}$ are identical according to the principle of the BG.

For one constitutive relation $e_{q}=y_{k}$, it can be expressed by $H_{m}^{r}(s) x=0$ where $H_{m}^{r}(s)$ is the $m$-th row of $H(s)$. In addition, note that the $m$-th entry of $F_{i}(s)$ is zero and thus $H_{m}^{r}(s) C=0$ obtained from (5.4). As a result, $c_{e_{q}}=c_{y_{k}}$. Similarly as $e_{q}=y_{k}$, the effort-equalized relations $e_{q}=e_{q+1}, \ldots,=e_{q+l-1}=y_{k}$ lead to

$$
c_{e_{q}}=c_{e_{q+1}}, \cdots,=c_{e_{q+l-1}}=c_{y_{k}}=0 .
$$

The further proof of this case needs another elementary matrix $\Sigma_{1}$ that represents operations of interchanging columns of $B_{s}^{n}(s)$ and fulfills $\Sigma_{1} \Sigma_{1}^{\top}=I_{2 n}$. Using $\Sigma_{1}$, rewriting (5.6) yields

$$
B_{s}^{n}(s) \Sigma_{1} \Sigma_{1}^{\top} C^{2 n}=F_{i}^{*}(s)
$$

where matrix $B_{s}^{n}(s) \Sigma_{1}$ has the following form

$$
B_{s}^{n}(s) \Sigma_{1}=2 n-2\left[\begin{array}{ccc} 
& c_{e_{q}} & c_{f_{q}} \\
2 n-1 & \mathbf{0}_{(2 n-3) \times 2} \\
B_{s}^{n-1}(s) & 0 & -1 \\
0 & -C s & 1 \\
\# & -1 & 0
\end{array}\right] .
$$

In (5.10), \# denotes a non-zero vector; and $B_{s}^{n-1}(s)$ denotes a square matrix that relates to the above mentioned $n$-bond graph without bond $q$. According to Lemma 
2.1, $B_{s}^{n-1}(s)$ has full rank since it depicts a $(n-1)$-bond graph. The last two columns of (5.10) correspond to bond $q$; and they are multiplied by the coefficients $c_{e_{q}}$ and $c_{f_{q}}$, respectively, as the two items in the expansion of (5.6). The $(2 n-2)$-th row of (5.10) depicts the flow-summed relation of this $\mathbf{0}$-junction (which relates to $\left.\sum_{t=0}^{l-1} f_{q+t}=0\right)$; the $(2 n-1)$-th row represents the constitutive relation of element $C$; and the $2 n$-th row denotes the effort-equalized relation between bond $q$ and another bond of this $\mathbf{0}$-junction. Let $U_{2}$ denote a row switching operation that interchanges the $(2 n-2)$-th and $2 n$-th rows in matrix $B_{s}^{n}(s) \Sigma_{1}$. Then, $B_{s}^{n}(s) \Sigma_{1}$ and $F_{i}^{*}(s)$ are left-multiplied by $U_{2}$, resulting in the following forms

$$
U_{2} B_{s}^{n}(s) \Sigma_{1}=2 n-2\left[\begin{array}{ccc}
c_{e_{q}} & c_{f_{q}} \\
2 n-1 & \mathbf{0}_{(2 n-3) \times 2} \\
B_{2 n-2}(s) & -1 & 0 \\
0 & -C s & 1 \\
\# & 0 & -1
\end{array}\right] \text { and } U_{2} F_{i}^{*}(s)=\left[\begin{array}{c}
\mathbf{0}_{(2 n-2) \times 1} \\
-1 \\
0
\end{array}\right]=F_{i}^{*}(s),
$$

where all the rows except the last one in the square matrix $B_{2 n-2}(s)$ are the same as those in matrix $B_{s}^{n-1}(s)$. Therefore, $\operatorname{Rank}\left[B_{2 n-2}(s)\right] \geq 2 n-3$. If $\operatorname{Rank}\left[B_{2 n-2}(s)\right]=$ $2 n-3$, then $\operatorname{Rank}\left[U_{2} B_{s}^{n}(s) \Sigma_{1}\right]<=2 n-1$, which contradicts the fact that $\operatorname{Rank}\left[U_{2} B_{s}^{n}(s) \Sigma_{1}\right]=$ 2n. As a result, $\operatorname{Rank}\left[B_{2 n-2}(s)\right]=2 n-2$. In the first $2 n-2$ rows of matrix $U_{2} B_{s}^{n}(s) \Sigma_{1}$, there has $B_{2 n-2} C^{2 n-2}+\left[\begin{array}{ll}\mathbf{0} & -1\end{array}\right]^{\top} c_{e_{q}}+\mathbf{0} c_{f_{q}}=\mathbf{0}$ where $C^{2 n-2}$ is the vector $\Sigma_{1}^{\top} C^{2 n}$ without the last two entries (i.e., $c_{e_{q}}$ and $c_{f_{q}}$ ). Straightforwardly, $C^{2 n-2}=\mathbf{0}$ since $c_{e_{q}}=0$ and $B_{2 n-2}$ has full rank. From the last row of matrix $U_{2} B_{s}^{n}(s) \Sigma_{1}$, it follows that $\# C^{2 n-2}-c_{f_{q}}=0$. Hence, $c_{f_{q}}=0$, resulting $C^{2 n}=\mathbf{0}$. Consequently, $B_{s}^{n}(s) C^{2 n}=\mathbf{0}$, which contradicts (5.6).

Next discuss the other case that the sensor variable $y_{k}$ and fault $f^{i}$ relate to two different junctions. Without loss of generality, it is assumed that fault $f^{i}$ occurs on 
a BG element $R$ which connects with a 1 -junction through a bond numbered $j$ and that the $y_{k}$ relates to a $\mathbf{0}$-junction which has $l$ bonds and connects with an inertia $C$ by a bond numbered $q$. Similar to the above case, in this $\mathbf{0}$-junction, all effort variables $e_{q}, e_{q+1}, \ldots, e_{q+l-1}$ are equal. Then, the following equalities hold

$$
c_{e_{q}}=c_{e_{q+1}}, \cdots,=c_{e_{q+l-1}}=c_{y_{k}}=0 .
$$

The subsequent proof demands another elementary matrix $\Sigma_{2}$ that represents operations of interchanging columns of $B_{s}^{n}(s)$ and fulfills $\Sigma_{2} \Sigma_{2}^{\top}=I_{2 n}$. With use of $\Sigma_{2},(5.6)$ is rewritten as follows

$$
B_{s}^{n}(s) \Sigma_{2} \Sigma_{2}^{\top} C^{2 n}=F_{i}^{*}(s)
$$

where matrix $B_{s}^{n}(s) \Sigma_{2}$ possesses the following form

$$
B_{s}^{n}(s) \Sigma_{2}=2 n-2\left[\begin{array}{ccc} 
& c_{e_{j}} & c_{f_{j}} \\
2 n-1 & \mathbf{0}_{(2 n-3) \times 2} \\
B_{s}^{n-1}(s) & -1 & 0 \\
0 & 1 & -R \\
\# & 0 & -1
\end{array}\right] .
$$

In (5.14), \# indicates a non-zero vector and $B_{s}^{n-1}(s)$ denotes a square matrix with rank $2 n-2$ that relates to the above mentioned $n$-bond graph without bond $j$. The last two columns of (5.14) correspond to bond $j$ and they are multiplied by the coefficients $c_{f_{j}}$ and $c_{e_{j}}$, respectively, as the two items of the expansion of (5.6). The $(2 n-2)$-th row of (5.14) means the sum of all efforts in the 1 -junction; the $(2 n-1)$-th row indicates the constitutive relation of element $R$ and the $2 n$-th row denotes the flow-equalized relation between bond $j$ and another bond of this 1-junction. The 
next context will give the proof of $B_{s}^{n}(s) C^{2 n}=\mathbf{0}$ under the condition (5.12).

The proof of $B_{s}^{n}(s) C^{2 n}=\mathbf{0}$ requires that $B_{s}^{n-1}(s) C^{2 n-2}=\mathbf{0}$ fulfills, where $C^{2 n-2}$ is a column-vector obtained by deleting the last two entries (i.e., $c_{f_{j}}$ and $c_{e_{j}}$ ) from $\Sigma_{2}^{\top} C^{2 n}$. From (5.6) and (5.14), note that

$$
B_{s}^{n-1}(s) C^{2 n-2}=\left[\begin{array}{c}
\mathbf{0}_{(2 n-3) \times 1} \\
c_{e_{j}}
\end{array}\right] .
$$

Let $F^{\#}=\left[\begin{array}{c}\mathbf{0}_{(2 n-3) \times 1} \\ c_{e_{j}}\end{array}\right]$. Similar to $\Sigma_{2}$, the proof of $B_{s}^{n-1}(s) C^{2 n-2}=\mathbf{0}$ needs two elementary matrices $\Sigma_{3}, U_{3}$. $\Sigma_{3}$ denotes operations of interchanging columns of $B_{s}^{n-1}(s)$ and satisfies $\Sigma_{3} \Sigma_{3}^{\top}=I_{2 n-2} ; U_{3}$ indicates operations of switching rows of $B_{s}^{n-1}(s)$ and has $U_{3} U_{3}^{\top}=I_{2 n-2}$. Rewriting $B_{s}^{n-1}(s) C^{2 n-2}$ using $\Sigma_{3}$ and $U_{3}$ yields

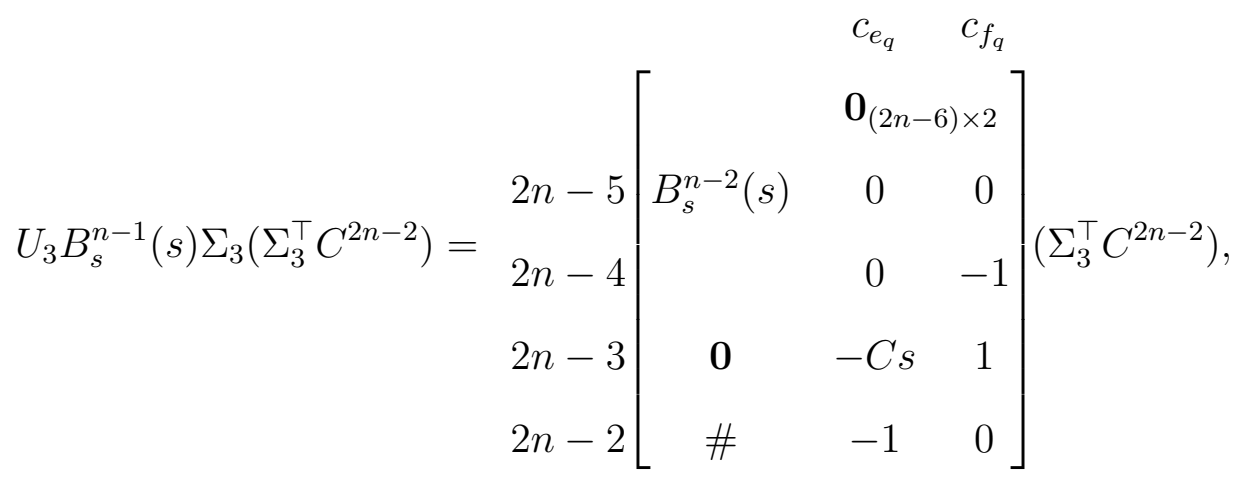

where \# denotes a non-zero vector and $B_{s}^{n-2}(s)$ relates to the above mentioned $n$ bond graph without bonds $q$ and $j$ as well as has full rank (i.e., $\operatorname{Rank}\left[B_{s}^{n-2}(s)\right]=2 n-$ $4)$. The $(2 n-2)$-th row in (5.16) shows the effort-equalized relation between bond $q$ and another bond of this $\mathbf{0}$-junction; the $(2 n-3)$-th row of matrix $U_{3} B_{s}^{n-1}(s) \Sigma_{3}$ represents the constitutive relation of element $C$; the $(2 n-4)$-th indicates the sum of all flows in the $\mathbf{0}$-junction that connects with $C$; and the $(2 n-5)$-th row in (5.16) closely relates to the $(2 n-2)$-th row in (5.14). Let $U_{4}$ represent a row 
switching operation that interchanges the $(2 n-5)$-th and $(2 n-2)$-th rows in matrix $U_{3} B_{s}^{n-1}(s) \Sigma_{3}$. Note that $U_{4} U_{3} F^{\#}=F^{\#}$. Additionally, left-multiplying matrix $U_{3} B_{s}^{n-1}(s) \Sigma_{3}$ by $U_{4}$ has the following form

$$
U_{4} U_{3} B_{s}^{n-1}(s) \Sigma_{3}=\begin{array}{r}
2 n-5 \\
2 n-4 \\
2 n-3 \\
2 n-2
\end{array}\left[\begin{array}{ccc} 
& c_{e_{q}} & c_{f_{q}} \\
& \mathbf{0}_{(2 n-6) \times 2} \\
B_{2 n-4}(s) & -1 & 0 \\
& 0 & -1 \\
0 & -C s & 1 \\
\# & 0 & 0
\end{array}\right],
$$

where all the rows except the next-to-last row in the square matrix $B_{2 n-4}(s)$ are the same as those in matrix $B_{s}^{n-2}(s)$. As a result, $\operatorname{Rank}\left[B_{2 n-4}(s)\right] \geq 2 n-5$. If $\operatorname{Rank}\left[B_{2 n-4}(s)\right]=2 n-5$, then $\operatorname{Rank}\left[U_{4} U_{3} B_{s}^{n-1}(s) \Sigma_{3}\right] \leq 2 n-3$, which contradicts $\operatorname{Rank}\left[U_{4} U_{3} B_{s}^{n-1}(s) \Sigma_{3}\right]=2 n-2$. Therefore, $\operatorname{Rank}\left[B_{2 n-4}(s)\right]=2 n-4$ (i.e., $B_{2 n-4}(s)$ has full rank). It follows from the $(2 n-3)$-th row of $(5.17)$ that $-C s c_{e_{q}}+c_{f_{q}}=0$. Due to $c_{e_{q}}=0, c_{f_{q}}=0$. From the first $2 n-4$ rows of $U_{4} U_{3} B_{s}^{n-1}(s) \Sigma_{3}$ in (5.17), it holds that $B_{2 n-4} C^{2 n-4}+\left[\begin{array}{lll}\mathbf{0} & -1 & 0\end{array}\right]^{\top} c_{e_{q}}+\left[\begin{array}{lll}\mathbf{0} & 0 & -1\end{array}\right]^{\top} c_{f_{q}}=\mathbf{0}$, where $C^{2 n-4}$ is a vector of the first $2 n-4$ entries in vector $\Sigma_{3}^{\top} C^{2 n-2}$ (without $c_{e_{q}}$ and $c_{f_{q}}$ ). Because $B_{2 n-4}(s)$ is full rank, $c_{f_{q}}=0$ and $c_{e_{q}}=0, C^{2 n-4}=\mathbf{0}$. As a result, $C^{2 n-2}=\mathbf{0}$ and thus $B_{s}^{n-1}(s) C^{2 n-2}=\mathbf{0}$. On returning to the proof of $B_{s}^{n}(s) C^{2 n}=\mathbf{0}$. Since $B_{s}^{n-1}(s) C^{2 n-2}=\mathbf{0}$, it is known from (5.15) that $c_{e_{j}}=0$. It follows from the $2 n$-th row in (5.14) that $\# C^{2 n-2}-c_{f_{j}}=0$. Therefore, $c_{f_{j}}=0$ since $C^{2 n-2}=\mathbf{0}$. As a consequence, $C^{2 n}=\mathbf{0}$. Then, $B_{s}^{n}(s) C^{2 n}=\mathbf{0} \neq F_{i}^{*}(s)$, which is a contradiction.

In summary, due to $C^{2 n}=\mathbf{0}$, it is known from (5.7) that $\left[c_{y_{1}}, c_{y_{2}}, \ldots, c_{y_{K}}\right]^{\top}=\mathbf{0}$ and thus $C=\mathbf{0}$. Therefore, $H(s) C=\mathbf{0} \neq F_{i}(s)$ which contradicts (5.4). This completes the proof. 
One may find that this lemma actually is a sufficient condition for detectability of any non-detectable fault. As a result of the above lemmas, a theorem of fault detectability can be represented in the DAEs model regarding to a DCBG.

Theorem 5.2. In the DAEs model (5.1) regarding to a DCBG, a non-detectable fault $f^{i}$ becomes detectable iff any sensor variable $y_{k} \in \mathcal{P}_{i}$ is measured by a sensor.

The proof is omitted since Lemmas 5.1 and 5.2 have proved the only-if part and the if part of this theorem, respectively.

\subsubsection{Optimization Problem}

The above subsection focuses on the necessary and sufficient condition of fault detectability in the DAEs model regarding to a DCBG. This subsection will present the necessary condition for distinguishability and formulate the optimization problem for sensor placement on the fault set $\mathcal{F}$.

Distinguishability of two faults is actually an ability of making one fault detectable from the model where the other fault has been detectable. To make a pair of faults distinguishable in a DAEs model, [89] also provides another theorem that is expressed by the below.

Theorem 5.3 (Frisk et al. [89]). Fault $f^{i}$ is distinguishable from fault $f^{j}$ in (5.1) iff $F_{i}(s) \notin \operatorname{Im}\left[H(s) F_{j}(s)\right]$.

Similar to Theorem 5.1, this theorem also can be preformed by the rank form of $\operatorname{Rank}\left[H(s) F_{i}(s) F_{j}(s)\right] \geq \operatorname{Rank}[H(s)]+2$. Following the spirit of Theorems 5.2, 5.3 and Lemma 5.1 , it is observed that a pair of sensor variables $\left(y_{k}, y_{k^{\prime}}\right), y_{k} \in$ $\mathcal{P}_{i}, y_{k^{\prime}} \in \mathcal{P}_{j}$, if measured by sensors, are possible to make two non-detectable faults distinguishable from each other. The following theorem will go into details of this observation. 
Theorem 5.4. Consider two non-detectable faults $f^{i}, f^{j} \in \mathcal{F}, i \neq j$, (i.e., $F_{i}(s), F_{j}(s) \in$ $\operatorname{Im}[H(s)])$ and their corresponding sets of sensor variables $\mathcal{P}_{i}, \mathcal{P}_{j} \subseteq \mathcal{P}$. According to Theorem 5.3, these two faults $f^{i}, f^{j}$ become distinguishable from each other iff

$$
\operatorname{Rank}\left[\begin{array}{ccc}
H(s) & F_{i}(s) & F_{j}(s) \\
\Theta_{\mathrm{sb}}\left(y_{k}\right) & 0 & 0 \\
\Theta_{\mathrm{sb}}\left(y_{k^{\prime}}\right) & 0 & 0
\end{array}\right] \geq \operatorname{Rank}\left[\begin{array}{c}
H(s) \\
\Theta_{\mathrm{sb}}\left(y_{k}\right) \\
\Theta_{\mathrm{sb}}\left(y_{k^{\prime}}\right)
\end{array}\right]+2 .
$$

If a pair of sensor variables $\left(y_{k}, y_{k^{\prime}}\right), y_{k} \in \mathcal{P}_{i}, y_{k^{\prime}} \in \mathcal{P}_{j}, y_{k} \neq y_{k^{\prime}}$, are measured by two sensors, then (5.18) holds necessarily.

Proof. Note that distinguishability two faults $f^{i}, f^{j}$ is symmetric, i.e., faults $f^{i}, f^{j}$ are distinguishable from each other. The proof of this theorem will be made from the point of fault $f^{j}$ distinguished from fault $f^{i}$. If any variable $y_{k} \in \mathcal{P}_{i}$ is measured by a sensor, Theorem 5.2 implies that fault $f^{i}$ is detectable. For ease of presentation, let $M\left(f^{i}\right)$ denote the DAEs model where fault $f^{i}$ is detectable. Fault $f^{j}$ distinguishable from fault $f^{i}$ is that fault $f^{j}$ is detectable from model $M\left(f^{i}\right)$. Lemma 5.1 implies that any variable $y_{k^{\prime}} \in \mathcal{P}_{j}$ measured is a necessary condition for detectability of fault $f^{j}$ from $M\left(f^{i}\right)$. Additionally, if $y_{k}=y_{k^{\prime}},(5.18)$ apparently violates. Therefore, a pair in $\left\{\left(y_{k}, y_{k^{\prime}}\right): y_{k} \in \mathcal{P}_{i}, y_{k^{\prime}} \in \mathcal{P}_{j}, y_{k} \neq y_{k^{\prime}}\right\}$ measured is a necessary condition to make(5.18) to fulfill. This ends the proof.

Theorem 5.4 shows the necessary condition to distinguish two non-detectable faults (i.e., to fulfill (5.18)). Let $\mathbb{Y}_{i, j}$ denote the set of all sensor variable pairs with respect to faults $f^{i}, f^{j}(i \neq j)$ where each $\left(y_{k}, y_{k^{\prime}}\right)$ is subject to $y_{k} \in \mathcal{P}_{i}, y_{k^{\prime}} \in$ $\mathcal{P}_{j}$ and $y_{k} \neq y_{k^{\prime}}$; and simultaneously let $\mathcal{D}_{i, j}$ represent a binary set where an element is equal to 1 if the corresponding pair $\left(y_{k}, y_{k^{\prime}}\right) \in \mathbb{Y}_{i, j}$ fulfills (5.18) and 0 otherwise. One must see that any set $\mathbb{Y}_{i, j}$ is a subset of $\mathbb{Y}=\left\{\left(y_{k}, y_{k^{\prime}}\right): y_{k}, y_{k^{\prime}} \in \mathcal{P}, 1 \leq k<\right.$ $\left.k^{\prime} \leq K\right\}$. To any pair of faults $f^{i}, f^{j}(i \neq j)$, if a pair $\left(y_{k}, y_{k^{\prime}}\right) \in \mathbb{Y} \backslash \mathbb{Y}_{i, j}$, the 
inequality (5.18) violates in the sense of fault distinguishability. This finding reveals that the binary set $\mathcal{D}_{i, j}$ with respect to a pair of faults $f^{i}, f^{j}$ can be easily extended to match with the set $\mathbb{Y}$. As a result, the extended $\mathcal{D}_{i, j}$ has the fixed cardinality $\left(\begin{array}{l}K \\ 2\end{array}\right)$. The following introduces how to set elements in the extended $\mathcal{D}_{i, j}$. When a pair $\left(y_{k}, y_{k^{\prime}}\right) \in \mathbb{Y} \backslash \mathbb{Y}_{i, j}$, the corresponding entry of $\mathcal{D}_{i, j}$ is directly set into 0; conversely, when a pair $\left(y_{k}, y_{k^{\prime}}\right) \in \mathbb{Y}_{i, j}$, the corresponding value is copied from the original $\mathcal{D}_{i, j}$. Finally, all these extended $\mathcal{D}_{i, j}{ }^{\prime} s$ can be stacked into the distinguishable matrix $\mathcal{D} \in\{0,1\}\left(\begin{array}{c}Q \\ 2\end{array}\right) \times\left(\begin{array}{c}K \\ 2\end{array}\right)$.

From the distinguishable matrix $\mathcal{D}$ viewpoint, fault isolability on the fault set $\mathcal{F}$ is achieved until an all-ones column is produced by uniting several columns in $\mathcal{D}$. However, in some systems, their distinguishable matrices $\mathcal{D}^{\prime} s$ show that not all pairs of faults are distinguishable. In other words, there exists a maximum-numberof-ones column which is generated by uniting all columns in $\mathcal{D}$. The $i$-th element in the maximum-number-of-ones column is mathematically defined as $\bigcup_{j=1}^{\left(K^{2}-K\right) / 2} d_{i j}$ where $d_{i j}$ is the $(i, j)$-th entry of matrix $\mathcal{D}$. Therefore, fault isolability in such systems is accomplished by producing the maximum-number-of-ones column, i.e., making a maximum number of pairs of faults distinguishable. To sum up the above stated two situations, fault isolability on the the fault set $\mathcal{F}$ studied in this chapter is to fulfill either (i) all pairs of faults or (ii) a maximum number of pairs of faults to be distinguishable. For the purpose of full/maximum isolability, a new set of sensor variables $\mathcal{Y}$ is introduced.

Definition 5.1. A set of sensor variables $\mathcal{Y}$ is an isolating set of sensor variables (ISSV) if for all (or a maximum number of) pairs of faults in $\left\{\left(f^{i}, f^{j}\right): f^{i}, f^{j} \in\right.$ $\mathcal{F}, 1 \leq i<j \leq Q\}$, the number of elements in $\left\{\left(y_{k}, y_{k^{\prime}}\right) \in \mathbb{Y}_{i, j}: y_{k}, y_{k^{\prime}} \in \mathcal{Y}\right\}$ that fulfill (5.18) is greater than zero.

An ISSV generically possesses a property of fulfilling fault isolability if all its members are measured by sensors, but a proper subset of it may also have this 
property. The minimal ISSVs are of special interest since these have attractive properties, like less cardinality, for fault isolability. The following characterizes these minimal ISSVs.

Definition 5.2. An ISSV $\mathcal{Y}$ is called a minimal isolating set of sensor variables (MISSV) if any proper subset of $\mathcal{Y}$ is not an ISSV.

Let $\mathbb{M}_{\mathcal{Y}}$ be the set of all possible MISSVs. The optimization problem can be formulated as

$$
\mathcal{Y}^{*}=\underset{\mathcal{Y} \in \mathbb{M}_{\mathcal{Y}}}{\arg \min }|\mathcal{Y}|
$$

where $\mathcal{Y}^{*}$ is the optimal set of sensor variables that will be measured by sensors in a DCBG.

From a general point of view, the optimization problem (5.19) belongs to the set covering problem. The successive section develops a DP algorithm to find all optimal solutions of this optimization problem.

\subsection{Dynamic Programming}

DP [124] is a powerful general technique for developing efficient optimization algorithms. In dynamic programming, let $s$ be a binary vector of size $\frac{Q^{2}-Q}{2}$ to indicate the distinguishability of all pairs of faults such that 1 means that a pair of faults are distinguishable from each other and 0 otherwise. For convenience, $s$ is called a state and let $\overrightarrow{\mathbf{1}}$ ( or $\overrightarrow{\mathbf{1}}_{m}$ ) be the state that all (or a maximum number of) pairs of faults are distinguishable. Let $\mathcal{Y}_{s}^{i} \subseteq \mathcal{P}$ indicate a set of sensor variables that has state $s$ and is produced by uniting $i$ pairs $\left(y_{k}, y_{k^{\prime}}\right)^{\prime} s \in \mathbb{Y}$ and let $\mathbb{Y}_{s}^{i}$ be the set of all possible $\mathcal{Y}_{s}^{i \prime} s$. For description of DP, a notation $L$ is introduced to denote the number of states associated with $\mathbb{Y}_{s}^{1 \prime} s$, i.e., $L=\left|\left\{s: \mathbb{Y}_{s}^{1} \neq \varnothing\right\}\right|$.

Note that the solution of (5.19), at worst, needs $\frac{K^{2}-K}{2}$ pairs $\left(y_{k}, y_{k^{\prime}}\right)$ united together. Then, the optimization problem (5.19) can be divided into $I=\min \left\{\frac{K^{2}-K}{2}, L\right\}$ 
subproblems. Each $i$-th subproblem of DP, $i=1,2, \ldots, I$, consists of sets $\mathbb{Y}^{D P}[i][s]^{\prime} s$, each set being associated with a state $s$ and a collection of sets $\mathcal{Y}_{s}^{i \prime} s$ with the same cardinality. Furthermore, let $G[i]$ denote the set of states associated with $\mathbb{Y}^{D P}[i][s]^{\prime} s$ at each subproblem, i.e., $G[i]=\left\{s: \mathbb{Y}^{D P}[i][s] \neq \varnothing\right\}$. More specifically, the initialization of the first subproblem (i.e., $i=1$ ) of DP can be expressed as follows

$$
\mathbb{Y}^{D P}[1][s]=\mathbb{Y}_{s}^{1}
$$

At each later $i$-th subproblem, $2 \leq i \leq I$, the DP update is

$$
\begin{array}{r}
\mathbb{Y}^{D P}[i][s]= \\
\underset{\mathcal{Y} \in \mathbb{Y}^{D P}[i-1][s] \text { and } \mathcal{Y}_{s^{\prime}}^{1} \cup \mathcal{Y}_{s^{\prime \prime}}^{i-1} \in \mathbb{Y}_{s}^{i}: \mathcal{Y}_{s^{\prime}}^{1} \in \mathbb{Y}^{D P}[1]\left[s^{\prime}\right], \mathcal{Y}_{s^{\prime \prime}}^{i-1} \in \mathbb{Y}^{D P}[i-1]\left[s^{\prime \prime}\right]}{\arg \min }\left\{|\mathcal{Y}|,\left|\mathcal{Y}_{s^{\prime}}^{1} \cup \mathcal{Y}_{s^{\prime \prime}}^{i-1}\right|\right\},
\end{array}
$$

where the term $\mathcal{Y}_{s^{\prime}}^{1} \cup \mathcal{Y}_{s^{\prime \prime}}^{i-1} \in \mathbb{Y}_{s}^{i}: \mathcal{Y}_{s^{\prime}}^{1} \in \mathbb{Y}^{D P}[1]\left[s^{\prime}\right], \mathcal{Y}_{s^{\prime \prime}}^{i-1} \in \mathbb{Y}^{D P}[i-1]\left[s^{\prime \prime}\right]$ means that the union of $\mathcal{Y}_{s^{\prime}}^{1}$ and $\mathcal{Y}_{s^{\prime \prime}}^{i-1}$ has the state $s=s^{\prime} \mid s^{\prime \prime}$ and $\mathcal{Y}_{s^{\prime}}^{1}, \mathcal{Y}_{s^{\prime \prime}}^{i-1}$ belong to $\mathbb{Y}_{s^{\prime}}^{1}, \mathbb{Y}_{s^{\prime \prime}}^{i-1}$, respectively. In (5.21), if the state $s$ associated with a set of sensor variables $\mathcal{Y}$ cannot be found in the $(i-1)$-th subproblem, i.e., $s \notin G[i-1]$, by default, consider $|\mathcal{Y}|=\infty$. Finally, the set $\mathbb{Y}^{*}$ of all optimal sets $\mathcal{Y}^{* \prime} s$ is given by

$$
\mathbb{Y}^{*}=\mathbb{Y}^{D P}[I][\overrightarrow{\mathbf{1}}] \text { or } \mathbb{Y}^{D P}[I]\left[\overrightarrow{\mathbf{1}}_{m}\right]
$$

$\mathbb{Y}^{D P}[I][\overrightarrow{\mathbf{1}}]$ (or $\mathbb{Y}^{D P}[I]\left[\overrightarrow{\mathbf{1}}_{m}\right]$ ) denotes the solution set where each member $\mathcal{Y}^{*}$ contains the least number of sensor variables and has the state $\overrightarrow{\mathbf{1}}$ (or $\overrightarrow{\mathbf{1}}_{m}$ ).

The above procedure for the optimization problem (5.19) can be interpreted by an algorithm in a formal and exhaustive fashion. Given a DAE model $M$, a set of faults $\mathcal{F}$ and a set of sensor variables $\mathcal{P}$, the pseudo-code to determine all optima of (5.19) is presented in Algorithm 5.1. 


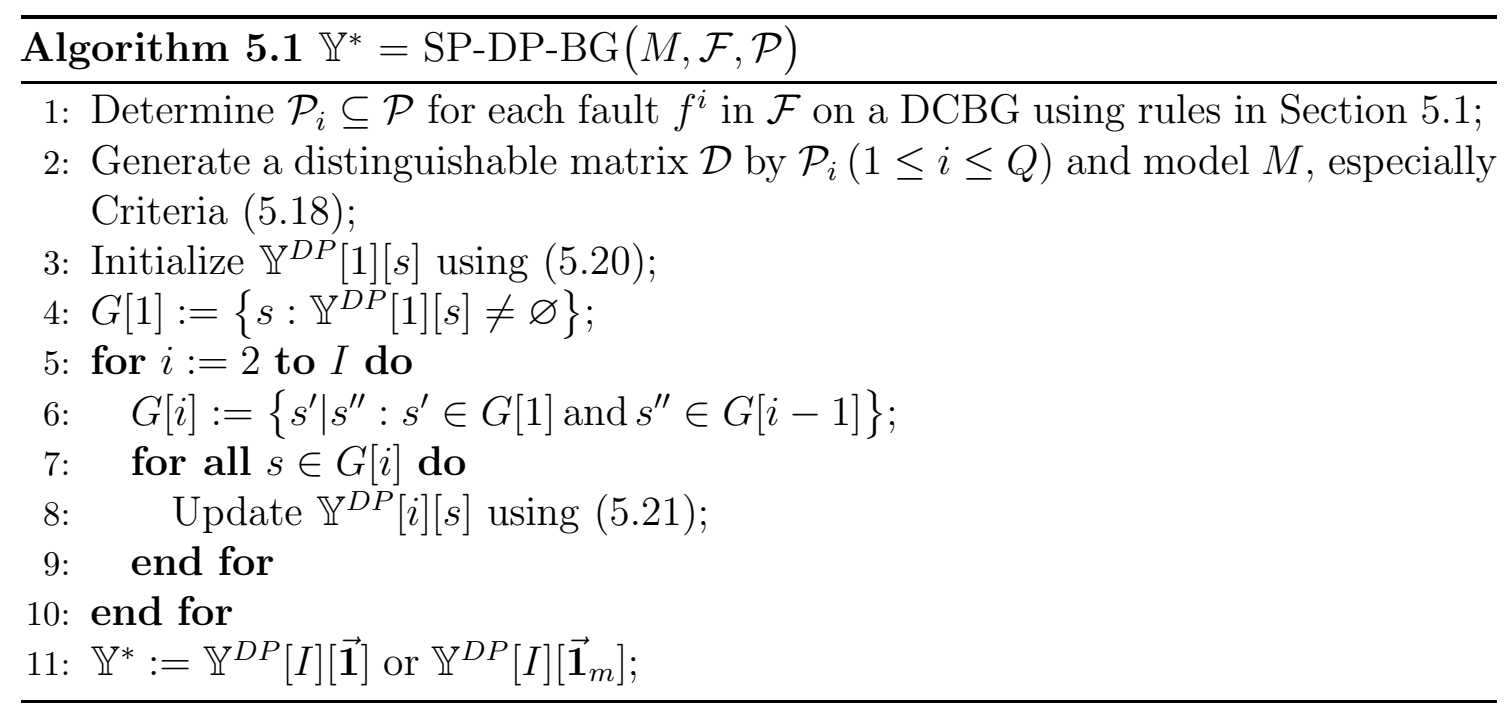

\subsection{Case Studies}

This section demonstrates this efficient sensor placement approach on three case studies: (a) a two-tank system, (b) a circuit and (c) a mechatronic system. In the case study (a), the two-tank system is employed to illustrate the proposed approach for sensor placement step by step. Then, the outcomes of this sensor placement approach are validated by deriving specific primary ARRs. The remaining case studies (b) and (c) are exemplified in Section 4.6 and employed to show the capability of efficiently placing sensors and the comparison of this approach with the one (i.e., SP-BG) presented in Chapter 4.

\subsubsection{A Two-tank System}

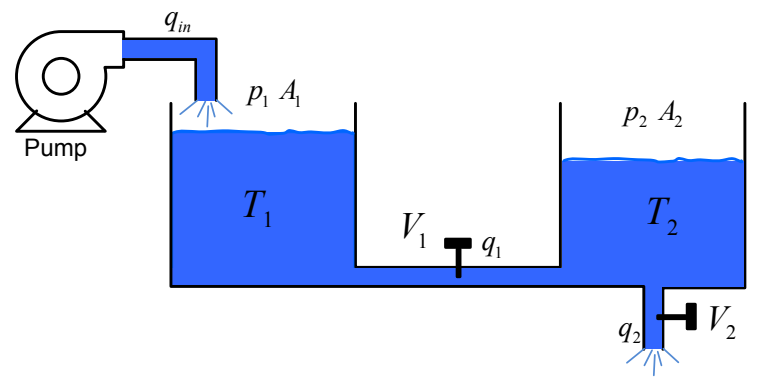

Figure 5.1: A two-tank system 
The two-tank system (see Figure 5.1) consists of two tanks, a regulated centrifugal pump modeled as a modulated source $q_{i n}$ and two valves represented by $V_{1}$ and $V_{2}$. In this system, assume that two valves are always open and their behaviors are linear, i.e., the amount of fluid passing a valve is proportional to the pressure drop at the valve. The first principle model describing the dynamics of this two-tank system are given as follows

$$
\begin{aligned}
& \frac{A_{1}}{g} \dot{p_{1}}=q_{\text {in }}-q_{1} \\
& \frac{A_{2}}{g} \dot{p_{2}}=q_{1}-q_{2} \\
& q_{1}=C d_{1}\left(p_{1}-p_{2}\right) \\
& q_{2}=C d_{2} p_{2},
\end{aligned}
$$

where $q_{i}(i=1,2)$ are mass flows, $p_{i}(i=1,2)$ pressures at the bottom of two tanks, $A_{i}(i=1,2)$ the cross-section areas of two tanks, $C d_{i}(i=1,2)$ coefficients of discharge of two valves, and $g$ the gravitational acceleration.

Figure 5.2 shows the DCBG of the two-tank system constructed using the procedure for hydraulic construction [54]. Two tanks are modeled as fluid capacitances, and the valves and pipes as fluid resistances. Parameters of this DCBG have $R_{V_{i}}=\frac{1}{C d_{i}}, C_{T_{i}}=\frac{A_{i}}{g}(i=1,2)$. This two-tank system considers four 1-port elements $\left\{C_{T_{1}}, R_{V_{1}}, C_{T_{2}}, R_{V_{2}}\right\}$ that become faulty during operation. In other words, there is a set $\mathcal{F}=\left\{f^{T_{1}}, f^{V_{1}}, f^{T_{2}}, f^{V_{2}}\right\}$ of four faults.

On the DCBG of the two-tank system, after applying the assignment rules of causal paths in Section 5.1, Figure 5.2 shows four causal paths regarding to faults in $\mathcal{F}=\left\{f^{T_{1}}, f^{V_{1}}, f^{T_{2}}, f^{V_{2}}\right\}$. To fault $f^{T_{1}}$, the causal path is $f^{T_{1}}\left(C_{T_{1}}\right) \rightarrow f_{2} \rightarrow \mathbf{0}_{1} \rightarrow$ $f_{3} \rightarrow \mathbf{1}_{1} \rightarrow f_{5} \rightarrow \mathbf{0}_{2}$ (which is the blue dotted line in Figure 5.2). Therefore, the corresponding subset of junctions is $\mathcal{J}_{T_{1}}=\left\{\mathbf{0}_{1}, \mathbf{1}_{1}, \mathbf{0}_{2}\right\}$. Applying the same procedure to faults in $\mathcal{F} \backslash\left\{f^{T_{1}}\right\}$, as a result, sets of junctions are $\mathcal{J}_{V_{1}}=\left\{\mathbf{0}_{1}, \mathbf{1}_{1}\right\}, \mathcal{J}_{T_{2}}=\left\{\mathbf{0}_{2}, \mathbf{1}_{2}\right\}$ and $\mathcal{J}_{V_{2}}=\left\{\mathbf{1}_{1}, \mathbf{0}_{2}, \mathbf{1}_{2}\right\}$. 


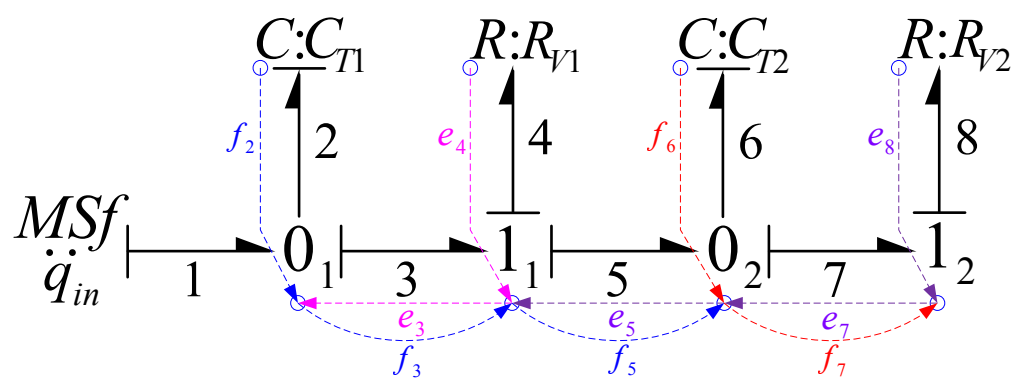

Figure 5.2: The DCBG of the two-tank system with four causal paths.

To establish the corresponding DAEs model of this two-tank system, junctions in $\mathcal{J}=\left\{\mathbf{1}_{1}, \mathbf{0}_{1}, \mathbf{1}_{2}, \mathbf{0}_{2}\right\}$ are assigned to sensor variables $y_{1}, y_{2}, y_{3}, y_{4}$ in sequence. Then, all subsets of sensor variables with respect to faults in $\mathcal{F}$ are $\mathcal{P}_{T_{1}}=\left\{y_{1}, y_{2}, y_{3}\right\}, \mathcal{P}_{V_{1}}=$ $\left\{y_{1}, y_{2}\right\}, \mathcal{P}_{T_{2}}=\left\{y_{3}, y_{4}\right\}$ and $\mathcal{P}_{V_{2}}=\left\{y_{2}, y_{3}, y_{4}\right\}$. As a result of pairwise combination of faults in $\mathcal{F}$, six sets of pairs of sensor variables are involved. They are enumerated as follows

$$
\begin{aligned}
& \mathbb{Y}_{T_{1}, V_{1}}=\left\{\left(y_{1}, y_{2}\right),\left(y_{1}, y_{3}\right),\left(y_{2}, y_{3}\right)\right\} \\
& \mathbb{Y}_{T_{1}, T_{2}}=\left\{\left(y_{1}, y_{3}\right),\left(y_{1}, y_{4}\right),\left(y_{2}, y_{3}\right),\left(y_{2}, y_{4}\right),\left(y_{3}, y_{4}\right)\right\}, \\
& \mathbb{Y}_{T_{1}, V_{2}}=\left\{\left(y_{1}, y_{2}\right),\left(y_{1}, y_{3}\right),\left(y_{1}, y_{4}\right),\left(y_{2}, y_{3}\right),\left(y_{2}, y_{4}\right),\left(y_{3}, y_{4}\right)\right\}, \\
& \mathbb{Y}_{V_{1}, T_{2}}=\left\{\left(y_{1}, y_{3}\right),\left(y_{1}, y_{4}\right),\left(y_{2}, y_{3}\right),\left(y_{2}, y_{4}\right)\right\}, \\
& \mathbb{Y}_{V_{1}, V_{2}}=\left\{\left(y_{1}, y_{2}\right),\left(y_{1}, y_{3}\right),\left(y_{1}, y_{4}\right),\left(y_{2}, y_{3}\right),\left(y_{2}, y_{4}\right)\right\} \text { and } \\
& \mathbb{Y}_{T_{2}, V_{2}}=\left\{\left(y_{2}, y_{3}\right),\left(y_{2}, y_{4}\right),\left(y_{3}, y_{4}\right)\right\} .
\end{aligned}
$$

Taking a pair of faults $f^{T_{1}}, f^{V_{1}}$ for example, the set $\mathbb{Y}_{T_{1}, V_{1}}$ with respect to them is $\left\{\left(y_{1}, y_{2}\right),\left(y_{1}, y_{3}\right),\left(y_{2}, y_{3}\right)\right\}$ and the respective binary set is $\mathcal{D}_{T_{1}, V_{1}}=\{1,1,0\}$. This example validates Theorem 5.4 that a pair $\left(y_{k}, y_{k^{\prime}}\right) \in \mathbb{Y}_{i, j}$ is necessary to fulfill (5.18). To extend all $\mathcal{D}_{i, j}{ }^{\prime} s$ with the same size $\left(\begin{array}{c}K \\ 2\end{array}\right)=\left(\begin{array}{l}4 \\ 2\end{array}\right)=6$ and then stack them together, the resulting distinguishable matrix $\mathcal{D}$ of the two-tank system arises and is shown in Table 5.1.

From the distinguishable matrix $\mathcal{D}$ shown in Table 5.1, note that both sets $\left(y_{1}, y_{4}\right)$ 
Table 5.1: A distinguishable matrix $\mathcal{D}$ of the two-tank system

\begin{tabular}{ccccccc}
\hline & $\left(y_{1}, y_{2}\right)$ & $\left(y_{1}, y_{3}\right)$ & $\left(y_{1}, y_{4}\right)$ & $\left(y_{2}, y_{3}\right)$ & $\left(y_{2}, y_{4}\right)$ & $\left(y_{3}, y_{4}\right)$ \\
\hline $\mathcal{D}_{T_{1}, V_{1}}$ & 1 & 1 & 0 & 0 & 0 & 0 \\
$\mathcal{D}_{T_{1}, T_{2}}$ & 0 & 1 & 1 & 1 & 1 & 0 \\
$\mathcal{D}_{T_{1}, V_{2}}$ & 1 & 1 & 1 & 1 & 1 & 1 \\
$\mathcal{D}_{V_{1}, T_{2}}$ & 0 & 1 & 1 & 1 & 1 & 0 \\
$\mathcal{D}_{V_{1}, V_{2}}$ & 0 & 1 & 1 & 1 & 1 & 0 \\
$\mathcal{D}_{T_{2}, V_{2}}$ & 0 & 0 & 0 & 0 & 1 & 1 \\
\hline
\end{tabular}

and $\left(y_{2}, y_{3}\right)$ are associated with a state $s=[011110]$. Accordingly, a total of five $\mathbb{Y}[1][s]^{\prime} s$ (i.e., $\mathbb{Y}_{s}^{1 \prime} s$ ) straightforwardly arise in the initialization of DP and they are enumerated as $\left\{\left(y_{1}, y_{2}\right)\right\},\left\{\left(y_{1}, y_{3}\right)\right\},\left\{\left(y_{1}, y_{4}\right),\left(y_{2}, y_{3}\right)\right\},\left\{\left(y_{2}, y_{4}\right)\right\}$ and $\left\{\left(y_{3}, y_{4}\right)\right\}$. Consequently, $L=5$. The above analysis indicates that the optimization problem of the two-tank system has $I=\min \left\{\left(\begin{array}{l}4 \\ 2\end{array}\right), 5\right\}=5$ subproblems. At the same time, the set of states in the first subproblem comes out to be $G[1]=\{[101000],[111110],-$ [011110], [011111], [001001]\}.

Clearly, the first subproblem does not contain the intended state $s^{*}$, i.e., $s^{*}=$ [111111], which shows that not all pairs of faults are distinguishable. To find the state $s^{*}$, there is a necessity of investigating the second iteration of DP. In comparison with $G[1]$, the set $G[2]$ increases two members, i.e., [111111] and [101001]. One can find that ISSVs in the second subproblem are $\left\{y_{1}, y_{3}, y_{4}\right\}$ and $\left\{y_{1}, y_{2}, y_{4}\right\}$. For the later subproblems of DP (i.e., $3 \leq i \leq 5$ ), their states stop increasing and ISSVs associated with the state $s^{*}$ are still $\left\{y_{1}, y_{3}, y_{4}\right\}$ and $\left\{y_{1}, y_{2}, y_{4}\right\}$. In conclusion, MISSVs of this two-tank systems are $\left\{y_{1}, y_{3}, y_{4}\right\}$ and $\left\{y_{1}, y_{2}, y_{4}\right\}$.

In the remainder of this section, a MISSV $\left\{y_{1}, y_{3}, y_{4}\right\}$ is arbitrarily chosen to verify whether it is the optimum of this two-tank system or not; in order to avoid repeatedly describing the similar verification, the other is left to readers. Figure 5.3 shows three sensors, $z_{1}, z_{2}$ and $z_{3}$, are already installed on the DCBG that measure sensor variables $y_{1}, y_{3}$ and $y_{4}$, respectively. Before deriving primary ARRs, a wellknown conclusion in the literature $[79,86,104,118]$ should be introduced that the 


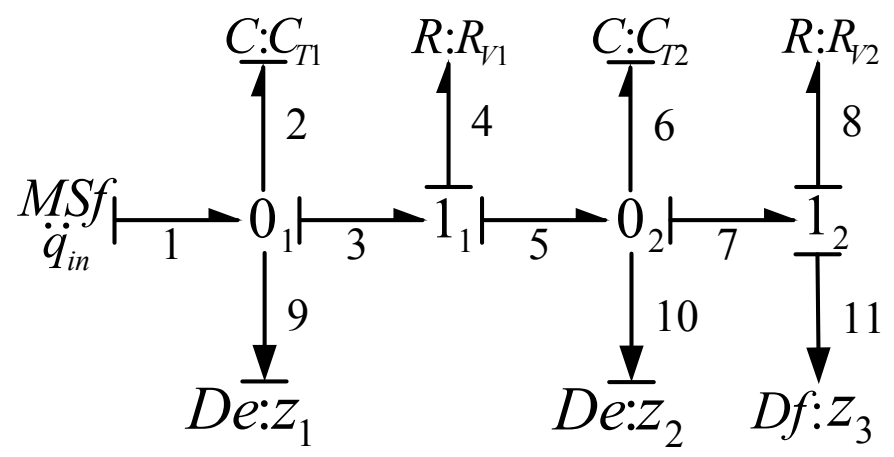

Figure 5.3: The DCBG of the two-tank system with three sensors

maximum number of primary ARRs is equal to the number of sensors in a BG model. Accordingly, this two-tank system has three primary ARRs. They are generated from the constitutive relations of BG elements $\left\{\mathbf{1}_{1}, \mathbf{0}_{2}, \mathbf{1}_{2}\right\}$.

For $\mathrm{BG}$ element $\mathbf{1}_{1}$, the constitutive relation of it is

$$
e_{3}-e_{4}-e_{5}=0
$$

where $e_{3}, e_{5}$ are measured by $z_{1}, z_{2}$ respectively. Hence $e_{3}=z_{1}$ and $e_{5}=z_{2}$. According to the constitutive relation on $R_{V_{1}}$, one can have $e_{4}=f_{4} R_{V_{1}}$, where $f_{4}=f_{3}=f_{1}-f_{2}$ following the causal path. Since $f_{1}=q_{\text {in }}$ and $f_{2}=C_{T_{1}} \dot{e}_{2}=C_{T_{1}} \dot{z}_{1}$, $f_{4}=q_{i n}-C_{T_{1}} \dot{z}_{1}$. These solutions lead (5.23) to the first ARR equation

$$
r_{1}=z_{1}-\left(q_{i n}-C_{T_{1}} \dot{z}_{1}\right) R_{V_{1}}-z_{2}
$$

For junction $\mathbf{0}_{2}$, its constitutive relation is

$$
f_{5}-f_{6}-f_{7}=0
$$

where $f_{7}$ is measured by $z_{3} ; f_{5}, f_{6}$ are two unknown variables. The constitutive relation of $C_{T_{2}}$ shows that $f_{6}=C_{T_{2}} \dot{e}_{6}$, where $e_{6}=z_{2}$. By covering the casual path, $f_{5}=f_{3}=q_{i n}-C_{T_{1}} \dot{z}_{1}$. Substituting these solutions into (5.25) yields the second 
Table 5.2: The FSM of this two-tank system

\begin{tabular}{c|cccc} 
& $f^{T_{1}}$ & $f^{V_{1}}$ & $f^{T_{2}}$ & $f^{V_{2}}$ \\
\hline$r_{1}$ & 1 & 1 & 0 & 0 \\
$r_{2}$ & 1 & 0 & 1 & 0 \\
$r_{3}$ & 0 & 0 & 0 & 1
\end{tabular}

ARR

$$
r_{2}=q_{\text {in }}-C_{T_{1}} \dot{z}_{1}-C_{T_{2}} \dot{z}_{2}-z_{3}
$$

Finally, the constitutive relation of junction $1_{2}$ is

$$
e_{7}-e_{8}=0
$$

where $e_{7}$ is measured by $z_{2}$. The constitutive relation of $R_{V_{2}}$ shows that $e_{8}=f_{8} R_{V_{2}}$, where $f_{8}=z_{3}$. Therefore, the third ARR is

$$
r_{3}=z_{2}-R_{V_{2}} z_{3}
$$

By collecting the fault information of these three ARRs, the fault signature matrix $[59,99]$ is built to present clearly the relationship between faults and ARRs, which is shown in Table 5.2. This fault signature matrix implies that each fault is isolable since each column is different from the rest. Therefore, it is proved that $\left\{y_{1}, y_{3}, y_{4}\right\}$ is exactly the optimal set for fault isolability in the two-tank system.

\subsubsection{Two Other Case Studies}

The circuit consists of a voltage source $V$, an internal resistor of the voltage source $r$, two resistors $R_{1}$ and $R_{2}$, an inductor $L$ and a capacitor $C$. Its DCBG is shown in Figure 4.7. As an example, the circuit has been studied in Chapter 4. Here, it is used to demonstrate the performance of the SP-DP-BG. Following two assignment rules of casual paths, six subsets of junctions are produced 
as $\mathcal{J}_{V}=\left\{\mathbf{1}_{1}\right\}, \mathcal{J}_{r}=\left\{\mathbf{1}_{1}, \mathbf{0}_{1}, \mathbf{1}_{2}\right\}, \mathcal{J}_{C}=\left\{\mathbf{0}_{1}, \mathbf{1}_{2}, \mathbf{0}_{2}\right\}, \mathcal{J}_{R_{1}}=\left\{\mathbf{1}_{1}, \mathbf{0}_{1}, \mathbf{1}_{2}\right\}, \mathcal{J}_{R_{2}}=$ $\left\{\mathbf{0}_{2}, \mathbf{1}_{3}\right\}$ and $\mathcal{J}_{L}=\left\{\mathbf{1}_{2}, \mathbf{0}_{2}, \mathbf{1}_{3}\right\}$. Each subset corresponds to faults in $\mathcal{F}_{\text {circuit }}=$ $\left\{f^{V}, f^{r}, f^{C}, f^{R_{1}}, f^{R_{2}}, f^{L}\right\}$. Then, the substitution of a junction (1 or $\left.\mathbf{0}\right)$ into a sensor variable $y_{k}$ is taken in $\mathcal{J}_{V}, \mathcal{J}_{r}, \mathcal{J}_{C}, \mathcal{J}_{R_{1}}, \mathcal{J}_{R_{2}}$ and $\mathcal{J}_{L}$. Next, the pairwise combination of faults in $\mathcal{F}_{\text {circuit }}$ is made, resulting in the following 15 sets:

$$
\begin{aligned}
& \mathbb{Y}_{V, r}=\left\{\left(y_{1}, y_{2}\right),\left(y_{1}, y_{3}\right)\right\} \\
& \mathbb{Y}_{V, C}=\left\{\left(y_{1}, y_{2}\right),\left(y_{1}, y_{3}\right),\left(y_{1}, y_{4}\right)\right\} \\
& \mathbb{Y}_{V, R_{1}}=\left\{\left(y_{1}, y_{2}\right),\left(y_{1}, y_{3}\right)\right\} \\
& \mathbb{Y}_{V, R_{2}}=\left\{\left(y_{1}, y_{4}\right),\left(y_{1}, y_{5}\right)\right\} \\
& \mathbb{Y}_{V, L}=\left\{\left(y_{1}, y_{3}\right),\left(y_{1}, y_{4}\right),\left(y_{1}, y_{5}\right)\right\} \\
& \mathbb{Y}_{r, C}=\left\{\left(y_{1}, y_{2}\right),\left(y_{1}, y_{3}\right),\left(y_{1}, y_{4}\right),\left(y_{2}, y_{3}\right),\left(y_{2}, y_{4}\right),\left(y_{3}, y_{4}\right)\right\} \\
& \mathbb{Y}_{r, R_{1}}=\left\{\left(y_{1}, y_{2}\right),\left(y_{1}, y_{3}\right),\left(y_{2}, y_{3}\right)\right\} \\
& \mathbb{Y}_{r, R_{2}}=\left\{\left(y_{1}, y_{4}\right),\left(y_{1}, y_{5}\right),\left(y_{2}, y_{4}\right),\left(y_{2}, y_{5}\right),\left(y_{3}, y_{4}\right),\left(y_{3}, y_{5}\right)\right\} \\
& \mathbb{Y}_{r, L}=\left\{\left(y_{1}, y_{3}\right),\left(y_{1}, y_{4}\right),\left(y_{1}, y_{5}\right),\left(y_{2}, y_{3}\right),\left(y_{2}, y_{4}\right),\left(y_{2}, y_{5}\right),\left(y_{3}, y_{4}\right),\left(y_{3}, y_{5}\right)\right\} \\
& \mathbb{Y}_{C, R_{1}}=\left\{\left(y_{1}, y_{2}\right),\left(y_{1}, y_{3}\right),\left(y_{1}, y_{4}\right),\left(y_{2}, y_{3}\right),\left(y_{2}, y_{4}\right),\left(y_{3}, y_{4}\right)\right\} \\
& \mathbb{Y}_{C, R_{2}}=\left\{\left(y_{2}, y_{4}\right),\left(y_{2}, y_{5}\right),\left(y_{3}, y_{4}\right),\left(y_{3}, y_{5}\right),\left(y_{4}, y_{5}\right)\right\} \\
& \mathbb{Y}_{C, L}=\left\{\left(y_{2}, y_{3}\right),\left(y_{2}, y_{4}\right),\left(y_{2}, y_{5}\right),\left(y_{3}, y_{4}\right),\left(y_{3}, y_{5}\right),\left(y_{4}, y_{5}\right)\right\} \\
& \mathbb{Y}_{R_{1}, R_{2}}=\left\{\left(y_{1}, y_{4}\right),\left(y_{1}, y_{5}\right),\left(y_{2}, y_{4}\right),\left(y_{2}, y_{5}\right),\left(y_{3}, y_{4}\right),\left(y_{3}, y_{5}\right)\right\} \\
& \mathbb{Y}_{R_{1}, L}=\left\{\left(y_{1}, y_{3}\right),\left(y_{1}, y_{4}\right),\left(y_{1}, y_{5}\right),\left(y_{2}, y_{3}\right),\left(y_{2}, y_{4}\right),\left(y_{2}, y_{5}\right),\left(y_{3}, y_{4}\right),\left(y_{3}, y_{5}\right)\right\} \\
&\left.\mathbb{P}_{R_{2}}\right)\left\{\left(y_{3}, y_{4}\right),\left(y_{3}, y_{5}\right),\left(y_{4}, y_{5}\right)\right\} .
\end{aligned}
$$

Applying Theorem 5.4 to the above 15 sets $\mathbb{Y}_{i, j}{ }^{\prime} s$ yields $15 \mathcal{D}_{i, j}{ }^{\prime} s$ and then extending these $15 \mathcal{D}_{i, j}{ }^{\prime} s$ gives rise to the corresponding distinguishable matrix $\mathcal{D}$ shown in Table 5.3. This table shows that there is a total of nine $\mathbb{Y}[1][s]^{\prime} s$ and therefore $L=9$. Specially, pairs $\left(y_{2}, y_{5}\right)$ and $\left(y_{3}, y_{4}\right)$ share a common state [000000011011110]. As 
Table 5.3: The distinguishable matrix $\mathcal{D}$ of the circuit presented in Section 4.6

\begin{tabular}{lcccccccccc}
\hline \multicolumn{7}{c}{$\left(y_{1}, y_{2}\right)\left(y_{1}, y_{3}\right)\left(y_{1}, y_{4}\right)\left(y_{1}, y_{5}\right)\left(y_{2}, y_{3}\right)$} & $\left(y_{2}, y_{4}\right)$ & $\left(y_{2}, y_{5}\right)$ & $\left(y_{3}, y_{4}\right)\left(y_{3}, y_{5}\right)\left(y_{4}, y_{5}\right)$ \\
\hline $\mathcal{D}_{V, r}$ & 0 & 0 & 0 & 0 & 0 & 0 & 0 & 0 & 0 & 0 \\
$\mathcal{D}_{V, C}$ & 1 & 1 & 1 & 0 & 0 & 0 & 0 & 0 & 0 & 0 \\
$\mathcal{D}_{V, R_{1}}$ & 1 & 1 & 0 & 0 & 0 & 0 & 0 & 0 & 0 & 0 \\
$\mathcal{D}_{V, R_{2}}$ & 0 & 0 & 1 & 1 & 0 & 0 & 0 & 0 & 0 & 0 \\
$\mathcal{D}_{V, L}$ & 0 & 1 & 1 & 1 & 0 & 0 & 0 & 0 & 0 & 0 \\
$\mathcal{D}_{r, C}$ & 1 & 1 & 1 & 0 & 0 & 0 & 0 & 0 & 0 & 0 \\
$\mathcal{D}_{r, R_{1}}$ & 1 & 1 & 0 & 0 & 1 & 0 & 0 & 0 & 0 & 0 \\
$\mathcal{D}_{r, R_{2}}$ & 0 & 0 & 1 & 1 & 0 & 1 & 1 & 1 & 1 & 0 \\
$\mathcal{D}_{r, L}$ & 0 & 1 & 1 & 1 & 1 & 1 & 1 & 1 & 1 & 0 \\
$\mathcal{D}_{C, R_{1}}$ & 0 & 1 & 1 & 0 & 1 & 1 & 0 & 0 & 0 & 0 \\
$\mathcal{D}_{C, R_{2}}$ & 0 & 0 & 0 & 0 & 0 & 1 & 1 & 1 & 1 & 0 \\
$\mathcal{D}_{C, L}$ & 0 & 0 & 0 & 0 & 1 & 1 & 1 & 1 & 1 & 1 \\
$\mathcal{D}_{R_{1}, R_{2}}$ & 0 & 0 & 1 & 1 & 0 & 1 & 1 & 1 & 1 & 0 \\
$\mathcal{D}_{R_{1}, L}$ & 0 & 0 & 1 & 1 & 0 & 1 & 1 & 1 & 1 & 0 \\
$\mathcal{D}_{R_{2}, L}$ & 0 & 0 & 0 & 0 & 0 & 0 & 0 & 0 & 1 & 1 \\
\hline
\end{tabular}

Table 5.4: The distinguishable matrix $\mathcal{D}$ of the mechatronic system presented in Section 4.6

\begin{tabular}{lcccccc}
\hline & $\left(y_{1}, y_{2}\right)$ & $\left(y_{1}, y_{3}\right)$ & $\left(y_{1}, y_{4}\right)$ & $\left(y_{2}, y_{3}\right)$ & $\left(y_{2}, y_{4}\right)$ & $\left(y_{3}, y_{4}\right)$ \\
\hline $\mathcal{D}_{V_{\text {in }}, L_{1}}$ & 0 & 1 & 1 & 1 & 1 & 0 \\
$\mathcal{D}_{V_{\text {in }}, L_{2}}$ & 1 & 1 & 1 & 1 & 1 & 1 \\
$\mathcal{D}_{L_{1}, L_{2}}$ & 0 & 0 & 0 & 0 & 1 & 1 \\
\hline
\end{tabular}

a result, the DP strategy in the SP-DP-BG has $I=\min \left\{\left(\begin{array}{l}5 \\ 2\end{array}\right), 9\right\}=9$ subproblems. After the SP-DP-BG is executed in this circuit, the only one MISSV is $\left\{y_{1}, y_{3}, y_{5}\right\}$, same as the result of the DP-BG.

As described in Section 4.6, the mechatronic system is composed of a voltage source $V_{i n}$, a resistor $R_{1}$, a rheostat $R_{2}$, an inductor $L_{1}$ and the motor shaft with the load $L_{2}$. The scheme of this mechatronic system is shown in Figure 4.8 and the corresponding DCBG with four causal paths is given in Figure 4.9. This mechatronic system concerns a fault set $\mathcal{F}_{\text {mech }}=\left\{f^{V_{i n}}, f^{L_{1}}, f^{L_{2}}\right\}$ and a set of four junctions $\left\{\mathbf{1}_{1}, \mathbf{0}_{1}, \mathbf{1}_{2}, \mathbf{1}_{3}\right\}$. Extracting junctions along the causal path regarding to each fault in $\mathcal{F}_{\text {mech }}$ yields the subsets of junctions $\mathcal{J}_{V_{\text {in }}}=\left\{\mathbf{1}_{1}, \mathbf{0}_{1}, \mathbf{1}_{2}, \mathbf{1}_{3}\right\}, \mathcal{J}_{L_{1}}=\left\{\mathbf{1}_{2}, \mathbf{1}_{3}\right\}$ and $\mathcal{J}_{L_{2}}=\left\{\mathbf{0}_{1}, \mathbf{1}_{2}, \mathbf{1}_{3}\right\}$. Substituting each junction into the corresponding sensor variable 
Table 5.5: The comparison between the SP-DP-BG and the SP-BG

\begin{tabular}{ccc}
\hline Case study & SP-DP-BG (sec) & SP-BG (sec) \\
\hline Circuit & 0.051 & 0.438 \\
Mechatronic system & 0.012 & 0.183 \\
\hline
\end{tabular}

$y_{k}$ and then taking the pairwise combination results in the following three sets:

$$
\begin{aligned}
& \mathbb{Y}_{V_{i n}, L_{1}}=\left\{\left(y_{1}, y_{3}\right),\left(y_{1}, y_{4}\right),\left(y_{2}, y_{3}\right),\left(y_{2}, y_{4}\right),\left(y_{3}, y_{4}\right)\right\} \\
& \mathbb{Y}_{V_{i n}, L_{2}}=\left\{\left(y_{1}, y_{2}\right),\left(y_{1}, y_{3}\right),\left(y_{1}, y_{4}\right),\left(y_{2}, y_{3}\right),\left(y_{2}, y_{4}\right),\left(y_{3}, y_{4}\right)\right\} \\
& \mathbb{Y}_{L_{1}, L_{2}}=\left\{\left(y_{2}, y_{3}\right),\left(y_{2}, y_{4}\right),\left(y_{3}, y_{4}\right)\right\}
\end{aligned}
$$

Theorem 5.4 is used to determine the distinguishability of each pair in $\mathbb{Y}_{V_{i n}, L_{1}}, \mathbb{Y}_{V_{i n}, L_{2}}$ and $\mathbb{Y}_{L_{1}, L_{2}}$. As a consequence, three sets $\mathcal{D}_{i, j}{ }^{\prime} s$ arise and then they are extended into forming a distinguishable matrix $\mathcal{D}$ of this mechatronic system, which is shown in Table 5.4. In the initialization of DP, four $\mathbb{Y}[1][s]^{\prime} s$ are $\left\{\left(y_{1}, y_{2}\right)\right\},\left\{\left(y_{1}, y_{3}\right),\left(y_{1}, y_{4}\right)\right.$,$\left.\left(y_{2}, y_{3}\right)\right\},\left\{\left(y_{2}, y_{4}\right)\right\}$ and $\left\{\left(y_{3}, y_{4}\right)\right\}$, respectively. Accordingly, $L=4$ and the DP consists of $I=\min \left\{\left(\begin{array}{l}4 \\ 2\end{array}\right), 4\right\}=4$ subproblems. The SP-DP-BG gives rise to the resultant MISSV $\left\{y_{2}, y_{4}\right\}$, which is the same as the finding of SP-BG.

The performance comparison between the SP-DP-BG and the SP-BG is conducted on a personal computer with the profile of which $\mathrm{OS}=$ Windows 7 , Memory $=8 \mathrm{~GB}$ and $\mathrm{CPU}=3.4 \mathrm{GHz}$. The compared result is in detail shown in Table 5.5. This table indicates that the SP-DP-BG perform better in terms of the efficiency.

\subsection{Summary}

This chapter concentrates on developing a more efficient method for sensor placement on BGs where 1-port elements behave linearly. Structural characteristics of BGs are further exploited and thus a simpler detectability condition has been derived. Drawing inspiration from the derivation of detectability, the necessity of 
distinguishability has been proposed and proved. The intention of this chapter is to distinguish all (a maximum of) pairs of faults. An optimization problem is formulated by collecting the results that arise from the simpler necessary condition of distinguishability (i.e., Theorem 5.4). This chapter designs a DP algorithm to fast obtain the optimal set of junctions. The representative case study of the two-tank system shows that the SP-DP-BG performs well. Meanwhile, two other case studies are employed to compare both the SP-DP-BG and the SP-BG performances. As a result, the SP-DP-BG performs better.

On comparing to the SPLCDP in Chapter 3, the SP-DP-BG obviates a timeconsuming step to generate a lot of residuals. Hence, the efficiency of the SP-DPBG is relatively higher. On comparing to the SP-BG in Chapter 4, the SP-DP-BG avoids solving the linear equations 5.2 for identifying the detectable sets with the aid of Theorem 5.2. As a consequence, the SP-DP-BG qualifies the efficient nature in the above stated sense. Moreover, the DP strategy is employed to search the optimal set of junctions, making the SP-DP-BG more efficient on placing sensors in linear and dynamic models. The disadvantage of the SP-DP-BG is the limitation of the applicable models since this approach is designed to handle linear and dynamic models. 


\section{Chapter 6}

\section{Conclusions and}

\section{Recommendations}

\subsection{Conclusions}

This dissertation concentrates on investigating the model-based sensor placement issues. Two types of definitions of fault detectability and isolability are considered to optimally place sensors in a continuous system. Three different sensor placement algorithms have been developed to achieve the maximum isolability requirement. The representative case studies reveal that the proposed algorithms all reach the desired optimality objectives. Some conclusions of this dissertation are summarized as follows:

i) A high-efficiency sensor placement algorithm has been developed to fulfill performance-based fault isolability. This sensor placement algorithm, called SPLCDP, is capable of making both system faults and sensor faults isolable. The core of the SPLCDP is an efficient search engine which is used to obtain all optimal MI sets. This efficient search engine has been realized by the LCDP. The complexity of the LCDP depends on the number of faults $Q$, the 
quantity of candidate sensors $K$ and the number of ARRs $N$. Particularly, the number of ARRs, which determines the number of state vectors in each $G[i]$ (i.e., $|G[i]|)$ in Algorithm 3.1, rests on the fault set $\mathcal{F}$ and the set $\mathcal{S}$. Due to the difficulty of calculating of the maximal number in $|G[i]|^{\prime} s$, the complexity of LCDP cannot be explicitly derived but the LCDP avoids falling into the complexity class NP. In addition, the complexity of the SPLCDP is affected by the procedure for $\mathcal{S}_{Q_{2}}$ in Section 3.4, whose complexity mainly depends on the cardinality of set $\mathcal{S} \backslash \mathcal{S}_{Q_{1}}$. In short, the complexity of the SPLCDP is closely related to the numbers of system faults and candidate sensors. However, most of the existing sensor placement methods, like the BILP, belong to the class NP with a complexity that depends exponentially with the sum of system faults and candidate sensors. Therefore, in the truck diesel engine system, the SPLCDP performs better than the BILP in the perspectives of the efficiency and the completeness of solutions. The SPLCDP is well suited to solve the sensor placement issues in linear or non-linear large systems.

ii) The properties of intrinsic fault detectability and isolability on a BG have been discussed. The conditions, which make a fault detectable and isolable, have been derived. The sensor placement algorithm (i.e., SP-BG) is designed on the basis of these conditions and it is applied to the mechatronic system example, showing excellent performance. As the main conclusion of the second algorithm, the sensor placement issue of the linear dynamic system is manifested from the intrinsic model property. This is a considerable progress to avoid deriving specific ARRs which are usually employed to develop sensor placement methods. Simultaneously, the SP-BG uses cause-effect relationships of model equations to guarantee enough primary ARRs for isolability. However, this sensor placement algorithm needs to invoke the basic building block (i.e., Algorithm 4.3) many times. This makes the computational cost expensive. 
iii) A more efficient method for sensor placement has been developed in the context of BG modelling. Based on the BG, causal path analysis is taken to determine which junctions can be used for isolability of a fault. Then the model property in terms of fault detectability is exploited. Detectability of fault $f^{i}$, which is proved from an algebraic point of view, is fulfilled if and only if any sensor variable in $\mathcal{P}_{i}$ with respect to this fault is measured by a sensor. The set $\mathcal{P}_{i}$ is extracted from the causal path for fault $f^{i}$ which is determined by an assignment rule. Therefore, on a BG, the fulfillment of detectability can be graphically interpreted that there exists one causal path from fault $f^{i}$ to a junction in $\mathcal{J}_{i}$. For distinguishability of two faults, the discussion on fault distinguishability in a DAEs model have been held. Theorem 5.4 exhibits the necessary condition for distinguishing two faults $f^{i}, f^{j}$. The BG explanation of this necessity is that there exist two causal paths which start from faults $f^{i}, f^{j}$ and end at two separate junctions in sets $\mathcal{J}_{i}, \mathcal{J}_{j}$, respectively. Based on the simpler necessity of distinguishability, the sensor placement algorithm has been redesigned for the purposes of maximum isolability and high-efficiency. The proposed sensor placement algorithm (i.e., SP-DP-BG) has been illustrated using the example of a two-tank system, showing that both high-efficiency and maximum isolability are achievable. It has been also necessary to show that for the two-tank system with a fault set $\mathcal{F}=\left\{f^{T_{1}}, f^{V_{1}}, f^{T_{2}}, f^{V_{2}}\right\}$, the set $\left\{y_{1}, y_{3}, y_{4}\right\}$ still is the optimal set if the nonlinear behaviours of two valves $V_{1}, V_{2}$ are under consideration. Therefore, the SP-DP-BG has demonstrated potentially to solve the sensor placement issues in nonlinear systems.

\subsection{Recommendations for Future Works}

Some future research works and possible extensions are identified as follows. 
i) Chapter 3 presents a sensor placement algorithm that is capable of finding out all possible solutions satisfying maximum isolability. In the sensor placement problem, besides maximum isolability, some other diagnostic requirements, like fault sensitivity and reliability, are possibly and necessarily considered in a real system $\Sigma$. Therefore, if some other requirements are involved, all global optima $\mathcal{X}^{* \prime} s$ which are only subject to the isolability requirement may not be the best and simultaneously the optimal set $\mathcal{S}_{Q_{2}}$ with the least number of candidate sensors may not be a superior choice. Since the set $\mathbb{X}^{*}$ computed by the SPLCDP contains all global optima, it is straightforward to pose an optimality condition regarding some other requirements to further determine ARRs and candidate sensors that will be installed in the system $\Sigma$. Therefore, one of future woks is to extend this algorithm applicable for more diagnostic requirements involved.

ii) Chapter 5 only presents the necessary condition for distinguishability of two faults. As a result, the proposed sensor placement algorithm (i.e., the SP-DPBG) has to enumerate all pairs of junctions to check whether or not faut distinguishability is satisfied. Note that if the necessary and sufficient condition for distinguishability of two faults is found, the sensor placement algorithm on the BG will be designed with more efficient performance. One future work would be to exploit the necessary and sufficient condition for distinguishability. For instance, consider a BG shown in Figure 5.2. Two subsets of junctions with respect to faults $f^{T_{1}}, f^{V_{1}}$ are $\mathcal{J}_{T_{1}}=\left\{\mathbf{0}_{1}, \mathbf{1}_{1}, \mathbf{0}_{2}\right\}$ and $\mathcal{J}_{V_{1}}=\left\{\mathbf{0}_{1}, \mathbf{1}_{1}\right\}$, respectively and thus the set $\mathbb{Y}_{T_{1}, V_{1}}$ is assigned into $\left\{\left(y_{1}, y_{2}\right),\left(y_{1}, y_{3}\right),\left(y_{2}, y_{3}\right)\right\}$. In Figure 5.2, causal paths from $f^{T_{1}}$ to each junction in $\mathcal{J}_{T_{1}}$ are listed as follows

$$
f^{T_{1}} \rightarrow f_{2} \rightarrow \mathbf{0}_{1}\left(y_{1}\right)
$$




$$
\begin{aligned}
& f^{T_{1}} \rightarrow f_{2} \rightarrow \mathbf{0}_{1}\left(y_{1}\right) \rightarrow f_{3} \rightarrow \mathbf{1}_{1}\left(y_{2}\right), \\
& f^{T_{1}} \rightarrow f_{2} \rightarrow \mathbf{0}_{1}\left(y_{1}\right) \rightarrow f_{3} \rightarrow \mathbf{1}_{1}\left(y_{2}\right) \rightarrow f_{5} \rightarrow \mathbf{0}_{2}\left(y_{3}\right) .
\end{aligned}
$$

Causal paths from $f^{V_{1}}$ to each junction in $\mathcal{J}_{V_{1}}$ are enumerated in the following

$$
\begin{aligned}
& f^{V_{1}} \rightarrow e_{4} \rightarrow \mathbf{1}_{1}\left(y_{2}\right), \\
& f^{V_{1}} \rightarrow e_{4} \rightarrow \mathbf{1}_{1}\left(y_{2}\right) \rightarrow e_{3} \rightarrow \mathbf{0}_{1}\left(y_{1}\right) .
\end{aligned}
$$

It is known from Chapter 5 that the pair $\left(y_{1}, y_{2}\right)$ is capable of making faults $f^{T_{1}}, f^{V_{1}}$ distinguishable. To the pair $\left(y_{1}, y_{2}\right)$, we find that Figure 5.2 exhibits two non-intersecting causal paths (6.1) and (6.4) for faults $f^{T_{1}}, f^{V_{1}}$, respectively. The pair $\left(y_{1}, y_{3}\right)$ also can make faults $f^{T_{1}}, f^{V_{1}}$ distinguishable. In the case of $\left(y_{1}, y_{3}\right)$, the causal path (6.3) with respect to $f^{T_{1}}$ and the causal path (6.4) intersects at junctions $\left\{\mathbf{0}_{1}, \mathbf{1}_{1}\right\}$. The pair $\left(y_{2}, y_{3}\right)$ fails to distinguish faults $f^{T_{1}}, f^{V_{1}}$. In Figure 5.2, two possible causal paths (6.3) and (6.5) with respect to $f^{T_{1}}, f^{V_{1}}$, respectively, intersect at junction $\mathbf{1}_{1}$. In summary, two faults are non-distinguishable if two causal paths intersects at one junction; on the other hand, two faults are distinguishable if two causal paths have zero or two intersecting junctions. Based on the above description, we attempt to propose the necessary and sufficient condition that two faults $f^{i}, f^{j}$ are distinguishable if two causal paths with respect to faults $f^{i}, f^{j}$ have even intersecting junctions. Proving the above proposed condition will be a possible future direction.

iii) Chapters 4 and 5 discuss the sensor placement issues without considering sensor faults. Therefore, one possible further work is to design a generalized fault isolability condition that sensor faults could be isolable.

iv) Chapters 4 and 5 present intrinsic fault detectability and isolability conditions for linear systems. Additionally, the BG modelling technique has an ability to 
model nonlinear systems. On a BG, another sensor placement issue arises as to how to derive conditions for intrinsic fault detectability and isolability in nonlinear systems. Due to the nonlinear property, the sensor placement issue is hard to handle. An applicable way is to extend the proposed approach to solve nonlinear sensor placement issues, which will be investigated in future. 


\section{Author's Publications}

\section{Journals:}

1. G. Chi, D. Wang, T. Le, M. Yu and M. Luo, "Sensor placement for fault isolability using low complexity dynamic programming", IEEE Transactions on Automation Science and Engineering (submitted)

2. G. Chi, D. Wang and S. Zhu, "An integrated approach for sensor placement in linear dynamic systems" Automatica (submitted)

3. G. Chi and D. Wang "Sensor placement for fault isolability on bond graph" IEEE Transactions on Automatic and Control (submitted)

\section{Conferences:}

1. G. Chi, T. Le, D. Wang, M. Yu, and M. Luo, "Sensor selection and placement using low complexity dynamic programming," in Proc. of IEEE Conference on Prognostics and Health Management, Denver, CO, USA, 2012.

2. G. Chi, D. Wang, M. Yu, M. Alavi, T. Le, and M. Luo, "Sensor placement for fault diagnosis using genetic algorithm," in Proc. of 17th IEEE Conference on Emerging Technologies and Factory Automation (ETFA2012), Krakow,Poland, 2012 .

3. G. Chi,W. Yan, and T. Chen, "Iterative data-based modelling and optimization for rapid design of dynamic processes," in Proc. of 9th IFAC Symposium 
on Dynamics and Control of Process Systems(DYCOPS2010), Leuven, Belgium, 2010, pp. 475-480. 


\section{Bibliography}

[1] A. Deraemaeker and K. Worden, New Trends in Vibration Based Structural Health Monitoring. Vienna: Springer, 2010.

[2] M. Blanke, M. Kinnaert, J. Lunze, and M. Staroswiecki, Diagnosis and FaultTolerant Control. Springer-Verlag, 2006.

[3] S. X. Ding, Model-based Fault Diagnosis Techniques: Design Schemes, Algorithms, and Tools. London: Springer, 2013.

[4] I. Hwang, S. Kim, Y. Kim, and C. Seah, "A survey of fault detection, isolation, and reconfiguration methods," IEEE Trans. Control Syst. Technol., vol. 18, no. 3, pp. 636-653, May 2010.

[5] J. Chen and R. J. Patton, Robust Model-based Fault Diagnosis for Dynamic Systems. Kluwer, 1999.

[6] J. Gertler, Fault Detection and Diagnosis in Engineering Systems. CRC press, 1998.

[7] J. Lu, A. P. Loh, and K. F. Fong, "Fault detectability and isolability conditions in the frequency domain," in Proc. of the IEEE Conference on Decision and Control, San Diego, CA, 2006, pp. 3518-3523.

[8] C. Edwards, S. K. Spurgeon, and R. J. Patton, "Sliding mode observers for fault detection and isolation," Automatica, vol. 36, no. 4, pp. 541-553, 2000.

[9] C. De Persis and A. Isidori, "A geometric approach to nonlinear fault detection and isolation," IEEE Trans. Autom. Control, vol. 46, no. 6, pp. 853 -865, Jun. 2001.

[10] R. Patton and J. Chen, "Observer-based fault detection and isolation: robustness and applications," Contr. Eng. Practice, vol. 5, no. 5, pp. 671-682, 1997.

[11] J. Gertler, "Analytical redundancy methods in fault detection and isolation," in Proc. of the IFAC/IMACS Symposium on Fault Detection, Supervision and Safety for Technical Processes (SAFEPROCESS'91), Baden-Baden, Germany, 1991, pp. 9-21. 
[12] M. Yu, D. Wang, M. Luo, D. Zhang, and Q. Chen, "Fault detection, isolation and identification for hybrid systems with unknown mode changes and fault patterns," Expert Syst. Appl., vol. 39, no. 11, pp. 9955-9965, 2012.

[13] W. Wang, L. Li, D. Zhou, and K. Liu, "Robust state estimation and fault diagnosis for uncertain hybrid nonlinear systems," Nonlinear analysis: Hybrid systems, vol. 1, no. 1, pp. 2-15, 2007.

[14] M. Desforges, P. Jacob, and A. Ball, "Fault detection in rotating machinery using kernel-based probability density estimation," International Journal of Systems Science, vol. 31, no. 11, pp. 1411-1426, 2000.

[15] P. Frank and N. Kiupel, "Fuzzy supervision and application to lean production," International journal of systems science, vol. 24, no. 10, pp. 1935-1944, 1993.

[16] N. Boudaoud and M. Masson, "Diagnosis of transient states: A pattern recognition approach," Journal Europeen des Systemes Automatises, vol. 34, no. 5, pp. 689-708, 2000.

[17] L. Chiang, M. Kotanchek, and A. Kordon, "Fault diagnosis based on Fisher discriminant analysis and support vector machines," Computers and Chemical Engineering, vol. 28, no. 8, pp. 1389-1401, 2004.

[18] V. Venkatasubramanian, R. Rengaswamy, K. Yin, and S. N. Kavuri, "A review of process fault detection and diagnosis: Part I: Quantitative model-based methods," Comput. Chem. Eng., vol. 27, no. 3, pp. 293 - 311, Mar. 2003.

[19] V. Venkatasubramanian, R. Rengaswamy, and S. N. Kavuri, "A review of process fault detection and diagnosis: Part II: Qualitative models and search strategies," Comput. Chem. Eng., vol. 27, no. 3, pp. 313-326, Mar. 2003.

[20] V. Venkatasubramanian, R. Rengaswamy, S. N. Kavuri, and K. Yin, "A review of process fault detection and diagnosis: Part III: Process history based methods," Comput. Chem. Eng., vol. 27, no. 3, pp. 327 - 346, Mar. 2003.

[21] C. Lo, Y. Wong, and A. Rad, "Intelligent system for process supervision and fault diagnosis in dynamic physical systems," IEEE Trans. Ind. Electron., vol. 53, no. 2, pp. 581-592, Apr. 2006.

[22] J.-F. Magni and P. Mouyon, "On residual generation by observer and parity space approaches," IEEE Trans. Autom. Control, vol. 39, no. 2, pp. 441-447, 1994.

[23] M. Yu, D. Wang, M. Luo, and L. Huang, "Prognosis of hybrid systems with multiple incipient faults: Augmented global analytical redundancy relations approach," IEEE Trans. Syst., Man, Cybern. A, vol. 41, no. 3, pp. 540-551, 2011. 
[24] S. J. Mason, "Feedback theory: Some properties of signal flow graphs," in Proc. of the I.R.E., vol. 41, no. 9, 1953, p. 11441156.

[25] P. J. Mosterman and G. Biswas, "Diagnosis of continuous valued systems in transient operating regions," IEEE Transactions on Systems, Man, and Cybernetics-Part A: Systems and Humans, vol. 29, no. 6, pp. 554-565, Nov. 1999.

[26] E. Manders, S. Narasimhan, G. Biswas, and P. Mosterman, "A combined qualitative/quantitative approach for efficient fault isolation in complex dynamic systems," in Proc. of 4th Symposium on Fault Detection, Supervision and Safety Processes, Budapest, Hungary, 2000, pp. 512-517.

[27] A. K. Samantaray and B. Ould-Bouamama, Model-based Process Supervision: A Bond Graph Approach. Springer, 2008.

[28] P. Frank and X. Ding, "Survey of robust residual generation and evaluation methods in observer-based fault detection systems," Journal of process control, vol. 7 , no. 6, pp. 403-424, 1997.

[29] R. PATTON and M. HOU, "Design of Fault Detection and Isolation Observers: A Matrix Pencil Approach," Automatica, vol. 34, no. 9, pp. 1135 - 1140, Sep. 1998.

[30] S. Simani, "Model-based Fault Diagnosis in Dynamic Systems Using Identification Techniques," Ph.D. dissertation, University of Modena and Reggio Emilia, 2003.

[31] R. Isermann, "Process fault detection based on modeling and estimation methodsa survey," Automatica, vol. 20, no. 4, pp. 387-404, 1984.

[32] M. S. Mahmoud and Y. Xia, Analysis and Synthesis of Fault-tolerant Control Systems. John Wiley \& Sons, 2013.

[33] A. Xu and Q. Zhang, "Nonlinear system fault diagnosis based on adaptive estimation," Automatica, vol. 40, no. 7, pp. 1181-1193, 2004.

[34] C. P. Tan and C. Edwards, "Multiplicative fault reconstruction using sliding mode observers," in Proc. of 5th Asian Control Conference, vol. 2, Melbourne, Australia, 2004, pp. 957-962.

[35] P. M. Frank and X. Ding, "Frequency domain approach to optimally robust residual generation and evaluation for model-based fault diagnosis," Automatica, vol. 30, no. 5, pp. 789-804, May 1994.

[36] K. Yin, "Minimax methods for fault isolation in the directional residual approach," Chem. Eng. Sci., vol. 53, no. 16, pp. 2921-2931, 1998. 
[37] M. Staroswiecki and G. Comtet-Varga, "Fault detectability and isolability in algebraic dynamic systems," in Proc. of the European Control Conference (ECC99), Karlsruhe, Germany, 1999.

[38] R. Merzouki, K. Fawaz, and B. Ould-Bouamama, "Hybrid fault diagnosis for telerobotics system," Mechatronics, vol. 20, no. 7, pp. 729-738, Oct. 2010.

[39] E. J. Manders, L. Barford, and G. Biswas, "An approach for fault detection and isolation in dynamic systems from distributed measurements," IEEE Trans. Instrum. Meas., vol. 51, no. 2, pp. 235-240, Apr. 2002.

[40] I. Roychoudhury, G. Biswas, and X. Koutsoukos, "Designing distributed diagnosers for complex continuous systems," IEEE Trans. Autom. Sci. Eng., vol. 6, no. 2, pp. 277-290, Apr. 2009.

[41] O. Adrot, D. Maquin, J. Ragot et al., "Fault detection with model parameter structured uncertainties," in European Control Conference (ECC99), Karlsruhe, Germany, 1999.

[42] O. Adrot, D. Maquin, and J. Ragot, "Diagnosis of an uncertain static system," in Proc. of the 39th IEEE Conference on Decision and Control, Sydney, Austrlia, 2000, pp. 4150-4154.

[43] M. A. Djeziri, R. Merzouki, and B. Ould Bouamama, "Robust monitoring of an electric vehicle with structured and unstructured uncertainties," IEEE Trans. Veh. Technol., vol. 58, no. 9, pp. 4710-4719, Nov. 2009.

[44] X. Ding, L. Guo, and T. Jeinsch, "A characterization of parity space and its application to robust fault detection," IEEE Transactions on Automatic Control, vol. 44, no. 2, pp. 337-343, Feb. 1999.

[45] X. Ding, L. Guo, and P. M. Frank, "Parameterization of linear observers and its application to observer design," IEEE Trans. Autom. Control, vol. 39, no. 8, pp. 1648-1652, Aug. 1994.

[46] A. Emami-Naeini, M. M. Akhter, and S. M. Rock, "Effect of model uncertainty on failure detection: The threshold selector," IEEE Trans. Autom. Control, vol. 33, no. 12, pp. 1106-1116, 1988.

[47] J. U. Thoma, Introduction to Bond Graphs and Their Applications. Oxford: Pergamon press, 1975.

[48] — Simulation by Bondgraphs: Introduction to a Graphical Method. Heidelberg: Springer-Verlag Berlin, 1990.

[49] A. Mukherjee and R. Karmakar, Modelling and Simulation of Engineering Systems through Bond Graphs. Pangbourne, UK: Alpha Science International, 2000. 
[50] A. Mukherjee and A. K. Samantaray, Bond Graph in Modeling, Simulation and Fault Identification. FL, USA: CRC Press, 2006.

[51] W. Borutzky, Bond graphs: A Methodology for Modelling Multidisciplinary Dynamic Systems. Erlangen, San Diego: SCS Publishing House, 2004.

[52] F. T. Brown, Engineering System Dynamics: a Unified Graph-Centered Approach. New York: CRC Press, 2007.

[53] F. E. Cellier, Continuous System Modelling. New York: Springer-Verlag, 1991.

[54] D. C. Karnopp, D. L. Margolis, and R. C. Rosenberg, System Dynamics: Modeling and Simulation of Mechatronic Systems, 4th ed. Hoboken, New Jersey: John Wiley \& Sons, 2006.

[55] M. A. Djeziri, B. Ould Bouamama, and R. Merzouki, "Modelling and robust FDI of steam generator using uncertain bond graph model," J. Process Control, vol. 19, no. 1, pp. 149-162, Jan. 2009.

[56] Y. Touati, R. Merzouki, and B. Ould-Bouamama, "Robust diagnosis to measurement uncertainties using bond graph approach: Application to intelligent autonomous vehicle," Mechatronics, vol. 22, no. 8, pp. 1148-1160, Dec. 2012.

[57] S. A. Arogeti, D. Wang, C. B. Low, and M. Yu, "Fault detection and isolation in a mobile robot test-bed," in IEEE/ASME International Conference on Advanced Intelligent Mechatronics, Singapore, Jul. 2009, pp. 398-404.

[58] C. B. Low, D. Wang, S. A. Arogeti, and M. Luo, "Quantitative hybrid bond graph-based fault detection and isolation," IEEE Trans. Autom. Sci. Eng., vol. 7, no. 3, pp. 558-569, Jul. 2010.

[59] C. B. Low, D. Wang, S. Arogeti, and J. B. Zhang, "Causality assignment and model approximation for hybrid bond graph: Fault diagnosis perspectives," IEEE Trans. Autom. Sci. Eng., vol. 7, no. 3, pp. 570-580, Jul. 2010.

[60] E. Chow and A. Willsky, "Analytical redundancy and the design of robust failure detection systems," IEEE Trans. Autom. Control, vol. 29, no. 7, pp. 603 - 614, Jul. 1984.

[61] A. K. Samantaray and S. K. Ghoshal, "Bicausal bond graphs for supervision: From fault detection and isolation to fault accommodation," J. Franklin Inst., vol. 345, no. 1, pp. 1-28, Jan. 2008.

[62] J. Gertler, "Fault detection and isolation using parity relations," Contr. Eng. Practice, vol. 5, no. 5, pp. 653-661, May 1997.

[63] R. Isermann, "Supervision, fault-detection and fault-diagnosis methods: An introduction," Contr. Eng. Practice, vol. 5, no. 5, pp. 639-652, May 1997. 
[64] M. J. Daigle, I. Roychoudhury, G. Biswas, X. D. Koutsoukos, A. PattersonHine, and S. Poll, "A comprehensive diagnosis methodology for complex hybrid systems: A case study on spacecraft power distribution systems," IEEE Trans. Syst., Man, Cybern. A, vol. 40, no. 5, pp. 917-931, Sep. 2010.

[65] M. J. Daigle, X. D. Koutsoukos, and G. Biswas, "Distributed diagnosis in formations of mobile robots," IEEE Trans. Robot., vol. 23, no. 2, pp. 353-369, Apr. 2007.

[66] M. Demetriou and M. Polycarpou, "Incipient fault diagnosis of dynamical systems using online approximators," IEEE Trans. Autom. Control, vol. 43, no. 11, pp. 1612-1617, Nov. 1998.

[67] R. Isermann and P. Ballé, "Trends in the application of model-based fault detection and diagnosis of technical processes," Contr. Eng. Practice, vol. 5, no. 5, pp. 709-719, May 1997.

[68] H. Ye, G. Wang, and S. Ding, "A new parity space approach for fault detection based on stationary wavelet transform," IEEE Trans. Autom. Control, vol. 49, no. 2, pp. 281-287, Feb. 2004.

[69] S. Ruan, Y. Zhou, F. Yu, K. Pattipati, P. Willett, and A. Patterson-Hine, "Dynamic multiple-fault diagnosis with imperfect tests," IEEE Trans. Syst., Man, Cybern. A, vol. 39, no. 6, pp. 1224-1236, Nov. 2009.

[70] M. Daigle, X. Koutsoukos, and G. Biswas, "A qualitative approach to multiple fault isolation in continuous systems," in Proc. of 22nd National Conference on Artificial Intelligence, Vancouver, Canada, 2007, pp. 293-298.

[71] M. Yu and D. Wang, "Model-based health monitoring for a vehicle steering system with multiple faults of unknown types," IEEE Trans. Ind. Electron., vol. 61, no. 7, pp. 3574-3586, 2014.

[72] M. Basseville, "On Fault Detectability and Isolability," European Journal of Control, vol. 7, no. 6, pp. 625 - 637, 2001.

[73] J. Gertler and D. Singer, "A new structural framework for parity equationbased failure detection and isolation," Automatica, vol. 26, no. 2, pp. 381-388, Mar. 1990.

[74] J. Chen and R. J. Patton, "A re-examination of fault detectability and isolability in linear dynamic systems," in Proc. of Fault Detection, Supervision and Safety for Technical Processes, Espoo, Finland, 1994, pp. 567-573.

[75] M. Nyberg and L. Nielsen, "A universal Chow-Willsky scheme and detectability criteria," IEEE Trans. Autom. Control, vol. 45, no. 1, pp. 152-156, Jan. 2000 . 
[76] J. Liu, J. L. Wang, and G.-H. Yang, "An LMI approach to minimum sensitivity analysis with application to fault detection," Automatica, vol. 41, no. 11, pp. 1995-2004, 2005.

[77] R. Raghuraj, M. Bhushan, and R. Rengaswamy, "Locating sensors in complex chemical plants based on fault diagnostic observability criteria," AlChE J., vol. 45, no. 2, pp. 310-322, Feb. 1999.

[78] M. Bhushan and R. Rengaswamy, "Design of sensor location based on various fault diagnostic observability and reliability criteria," Comput. Chem. Eng., vol. 24, no. 2-7, pp. 735-741, Jul. 2000.

[79] L. Travé-Massuyès, T. Escobet, and X. Olive, "Diagnosability analysis based on component-supported analytical redundancy relations," IEEE Trans. Syst., Man, Cybern. A, vol. 36, no. 6, pp. 1146-1160, Nov. 2006.

[80] F. Nejjari, R. Sarrate, and A. Rosich, "Optimal sensor placement for fuel cell system diagnosis using BILP formulation," in Proc. of 18th IEEE Mediterranean Conference on Control and Automation (MED'10), Marrakech, Morocco, 2010, pp. 1296-1301.

[81] R. Sarrate, V. Puig, T. Escobet, and A. Rosich, "Optimal sensor placement for model-based fault detection and isolation," in Proc. of 46th IEEE CDC, New Orleans, LA, USA, 2007, pp. 2584-2589.

[82] M. Krysander, J. Åslund, and M. Nyberg, "An efficient algorithm for finding minimal overconstrained subsystems for model-based diagnosis," IEEE Trans. Syst., Man, Cybern. A, vol. 38, no. 1, pp. 197-206, Feb. 2008.

[83] M. Krysander, "Design and analysis of diagnosis systems using structural methods," Ph.D. dissertation, Linköping Univ., Linköping, Sweden, Jun. 2006.

[84] A. Rosich, E. Frisk, J. Åslund, R. Sarrate, and F. Nejjari, "Fault diagnosis based on causal computations," IEEE Trans. Syst., Man, Cybern. A, vol. 42, no. 2, pp. 371-381, Mar. 2012.

[85] C. Commault and J.-M. Dion, "Sensor location for diagnosis in linear systems: A structural analysis," IEEE Trans. Autom. Control, vol. 52, no. 2, pp. 155169, Feb. 2007.

[86] M. Staroswiecki and G. Comtet-Varga, "Analytical redundancy relations for fault detection and isolation in algebraic dynamic systems," Automatica, vol. 37, no. 5, pp. 687-699, May 2001.

[87] C. Commault, J.-M. Dion, and S. Y. Agha, "Structural analysis for the sensor location problem in fault detection and isolation," Automatica, vol. 44, no. 8, pp. 2074-2080, Aug. 2008. 
[88] — - "Structural analysis for the sensor location problem in fault detection and isolation," in Proc. of 6th IFAC Symp. SafeProcess, Beijing, China, 2006, pp. 896-901.

[89] E. Frisk, M. Krysander, and J. Åslund, "Sensor placement for fault isolation in linear differential-algebraic systems," Automatica, vol. 45, no. 2, pp. 364-371, Feb. 2009.

[90] M. Krysander and E. Frisk, "Sensor placement for fault diagnosis," IEEE Trans. Syst., Man, Cybern. A, vol. 38, no. 6, pp. 1398-1410, Nov. 2008.

[91] M. Basseville, A. Benveniste, G. V. Moustakides, and A. Rougee, "Optimal sensor location for detecting changes in dynamical behavior," IEEE Trans. Autom. Control, vol. 32, no. 12, pp. 1067-1075, Dec. 1987.

[92] R. Debouk, S. Lafortune, and D. Teneketzis, "On an optimization problem in sensor selection for failure diagnosis," in Proc. of 38th IEEE CDC, Phoenix, AZ, USA, 1999, pp. 4990-4995.

[93] — - "On an optimization problem in sensor selection," Discrete Event Dyn. Syst., vol. 12, no. 4, pp. 417-445, Oct. 2002.

[94] L. Hedjazi, T. Kempowsky-Hamon, L. Despènes, M.-V. Le Lann, S. Elgue, and J. Aguilar-Martin, "Sensor placement and fault detection using an efficient fuzzy feature selection approach," in Proc. of the 49th IEEE Conference on Decision and Control, Atlanta, GA,USA, 2010, pp. 6827-6832.

[95] P. Sadegh and J. C. Spall, "Optimal sensor configuration for complex systems," in Proc. of the Americam Control Conference, Philadelphia, Pennsylvania, 1998, pp. 376-380.

[96] T. Boukhobza and F. Hamelin, "Observability analysis and sensor location study for structured linear systems in descriptor form with unknown inputs," Automatica, vol. 47, no. 12, pp. 2678-2683, Dec. 2011.

[97] T. Boukhobza, F. Hamelin, G. Kabadi, and S. Aberkane, "Discrete mode observability of switching linear systems with unknown inputs: A graph-theoretic approach," in Proc. of 18th IFAC World Congress, Milano, Italy, 2011, pp. 6616-6621.

[98] T. Boukhobza, "Sensor location for discrete mode observability of switching linear systems with unknown inputs," Automatica, vol. 48, no. 7, pp. 12621272, Jul. 2012.

[99] G. Chi, T. Le, D. Wang, M. Yu, and M. Luo, "Sensor selection and placement using low complexity dynamic programming," in Proc. of IEEE Conference on Prognostics and Health Management, Denver, CO, USA, 2012. 
[100] S. Arogeti, D. Wang, C. B. Low, and M. Luo, "Energy-Based Mode Tracking of Hybrid Systems for FDI," IEEE Trans. Syst., Man, Cybern., Syst., vol. 43, no. 1, pp. 14-28, Jan. 2013.

[101] M. Yu, M. Luo, D. Wang, S. A. Arogeti, and X. Zhang, "Simultaneous fault and mode switching identification for hybrid systems based on particle swarm optimization," Expert Syst. Appl., vol. 37, no. 4, pp. 3000-3012, Apr. 2010.

[102] S. A. Arogeti, D. Wang, and C. B. Low, "Mode identification of hybrid systems in the presence of fault," IEEE Trans. Ind. Electron., vol. 57, no. 4, pp. 1452 1467, Apr. 2010.

[103] B. Ould-Bouamama, R. El Harabi, M. Abdelkrim, and M. Ben Gayed, "Bond graphs for the diagnosis of chemical processes," Comput. Chem. Eng., vol. 36, no. 0, pp. 301-324, 2012.

[104] A. K. Samantaray, K. Medjaher, B. Ould Bouamama, M. Staroswiecki, and G. Dauphin-Tanguy, "Diagnostic bond graphs for online fault detection and isolation," Simulation Modelling Practice and Theory, vol. 14, no. 3, pp. 237262, Apr. 2006.

[105] H. M. Paynter, "Hydraulics by analog-An electronic model of a pumping plant," Journal Boston Society of Civil Engineering, pp. 197-219, Jul. 1959.

[106] — - Analysis and Design of Engineering Systems. MIT Press, 1961.

[107] R. Rosenberg and D. Karnopp, "A definition of the bond graph language," Journal of Dynamic Systems, Measurement, and Control, vol. 94, p. 179, 1972.

[108] R. C. Rosenberg, "Computer-aided Teaching of Dynamic System Behavior," Ph.D. dissertation, Massachusetts Institute of Technology, 1965.

[109] D. C. Karnopp and R. C. Rosenberg, Analysis and Simulation of Multiport Systems: The Bond Graph Approach to Physical System Dynamics. Mass: MIT Press Cambridge, 1968.

[110] D. C. Karnopp, D. L. Margolis, and R. C. Rosenberg., System Dynamics:Modeling and Simulation, and Control of Mechatronic Systems, 5th ed. Hoboken, New Jersey: John Wiley \& Sons, 2012.

[111] M. Tagina, J. Cassar, G. Dauphin-Tanguy, and M. Staroswiecki, "Monitoring of systems modelled by bond-graphs," in Proc. of the 1995 International Conference on Bond Graph Modeling and Simulation (ICBGM'95), Las Vegas, Nevada, 1995, pp. 275-279.

[112] B. Ould-Bouamama, A. K. Samantaray, M. Staroswiecki, and G. DauphinTanguy, "Derivation of constraint relations from bond graph models for fault detection and isolation," in Proc. of the 2003 International Conference on Bond Graph Modeling and Simulation (ICBGM'03), Orlando, Florida, 2003, pp. 104-109. 
[113] A. Rosich, R. Sarrate, and F. Nejjari, "Optimal sensor placement for FDI using binary integer linear programming," in 20th International Workshop on Principles of Diagnosis, DX09, 2009.

[114] V. Venkatasubramanian, R. Rengaswamy, K. Yin, and S. N. Kavuri, "A review of process fault detection and diagnosis: Part I: Quantitative model-based methods," Comput. Chem. Eng., vol. 27, no. 3, pp. 293-311, Mar. 2003.

[115] C. Svärd, M. Nyberg, E. Frisk, and M. Krysander, "Automotive engine FDI by application of an automated model-based and data-driven design methodology," Contr. Eng. Practice, vol. 21, no. 4, pp. 455-472, Apr. 2013.

[116] J. Wahlström and L. Eriksson, "Modelling diesel engines with a variablegeometry turbocharger and exhaust gas recirculation by optimization of model parameters for capturing non-linear system dynamics," Proc. of the Institution of Mechanical Engineers, Part D: Journal of Automobile Engineering, vol. 225, no. 7, pp. 960-986, Jul. 2011.

[117] The ILOG website. [Online]. Available: http://www.ilog.com

[118] A. K. Samantaray, K. Medjaher, B. Ould Bouamama, M. Staroswiecki, and G. Dauphin-Tanguy, "Component-based modelling of thermofluid systems for sensor placement and fault detection," Simulation, vol. 80, no. 7-8, pp. 381398, Aug. 2004.

[119] B. Ould Bouamama, K. Medjaher, M. Bayart, A. K. Samantaray, and B. Conrard, "Fault detection and isolation of smart actuators using bond graphs and external models," Contr. Eng. Practice, vol. 13, no. 2, pp. 159-175, 2005.

[120] R. Cacho, J. Felez, and C. Vera, "Deriving simulation models from bond graphs with algebraic loops: The extension to multibond graph systems," $J$. Franklin Inst., vol. 337, no. 5, pp. 579-600, Aug. 2000.

[121] M. Nyberg and E. Frisk, "Residual generation for fault diagnosis of systems described by linear differential-algebraic equations," IEEE Trans. Autom. Control, vol. 51, no. 12, pp. 1995-2000, Dec. 2006.

[122] J. W. Polderman and J. C. Willems, Introduction to Mathematical Systems Theory: A Behavioral Approach. New York: Springer, 1997.

[123] V. Chvatal, "A greedy heuristic for the set-covering problem," Mathematics of operations research, vol. 4, no. 3, pp. 233-235, Aug. 1979.

[124] R. E. Bellman and S. E. Dreyfus, Applied dynamic programming, 2nd ed. Princeton, NJ: Princeton Univ. Press, 1962. 


\section{Appendix}

- DC-motor and pump

$$
\begin{aligned}
k_{1} k_{2} u_{i n} & =J_{1} \dot{\omega}_{1}+g_{2}\left(R_{2 v}, R_{2 c}, \omega_{1}\right)+\tau_{O} \\
\omega_{2} & =k_{3} k_{4} \omega_{1} \\
\dot{\theta_{1}} & =k_{3} \omega_{1} \\
\tau_{P_{i}} & =\frac{\tau_{O}}{k_{3} k_{4}} \\
\tau_{P_{i}} & =J_{2} \dot{\omega}_{2}+g_{3}\left(R_{3 v}, R_{3 c}, \omega_{2}\right)+\tau_{P_{o}}
\end{aligned}
$$

- Hydraulic cylinder

$$
\begin{aligned}
\tau_{P_{o}} & =k_{5}\left(p_{1}-p_{2}\right) \\
\omega_{2} & =\frac{q_{1}}{k_{5}} \\
q_{1} & =A v_{\text {pist }}+\operatorname{sign}\left(p_{1}-p_{2}\right) \sqrt{\left|p_{1}-p_{2}\right|}\left(C d+f^{\text {pist }}\right) / g \\
F_{\text {pist }} & =A\left(p_{1}-p_{2}\right)
\end{aligned}
$$

- Ackerman's steering mechanism

$$
\begin{aligned}
F_{\text {pist }} & =\frac{\tau_{W 1}}{F_{1}\left(\theta_{2}\right)} \\
v_{\text {pist }} & =F_{1}\left(\theta_{2}\right) \omega_{3} \\
\omega_{4} & =\frac{\omega_{3}}{F_{2}\left(\theta_{2}\right)} \\
\tau_{W 2} & =F_{2}\left(\theta_{2}\right) \tau_{W 1} \\
\dot{\theta_{2}} & =\omega_{3}
\end{aligned}
$$


Table A.1: Functions used in the front steering model

\begin{tabular}{ll}
\hline Function & Description \\
\hline$g_{2}(\cdot)$ & the DC-motor friction torque. \\
$g_{3}(\cdot)$ & the pump friction torque. \\
$F_{1}\left(\theta_{2}\right)$ & the nonlinear transformer modulus with respect to $\theta_{2}$. \\
$F_{2}\left(\theta_{2}\right)$ & the nonlinear transformer modulus with respect to $\theta_{2}$. \\
$g_{7}(\cdot)$ & the friction torque loss \\
\hline
\end{tabular}

- Wheel

$$
\tau_{W 2}=J_{3} \dot{\omega}_{4}+g_{7}\left(R_{7 v}+f^{\text {tire }}, R_{7 c}+f^{\text {tire }}, \omega_{4}\right)
$$

- Sensors

$$
\begin{aligned}
s_{\theta_{1}} & =\theta_{1}+f_{s}^{\theta_{1}} \\
s_{\theta_{2}} & =\theta_{2}+f_{s}^{\theta_{2}} \\
s_{p_{1,2}} & =p_{1}-p_{2}+f_{s}^{p_{1,2}} \\
s_{\omega_{1}} & =\omega_{1}+f_{s}^{\omega_{1}} \\
s_{\omega_{3}} & =\omega_{3}+f_{s}^{\omega_{3}} \\
s_{q_{1}} & =q_{1}+f_{s}^{q_{1}}
\end{aligned}
$$

Among the above 21 equations, equations $\{A .1, A .5, A .8, A .10, A .11, A .12, A .13, A .15\}$ are nonlinear and the rest are linear. $g_{2}(\cdot), g_{3}(\cdot), g_{7}(\cdot), F_{1}(\cdot), F_{2}(\cdot)$ are nonlinear functions describing the dynamics of the front steering system and recapitulated in Table A.1.

ARRs in the optimal MI set $\mathcal{X}^{*}$ exemplified in Table 3.8:

$r_{107}=\{A .1, A .2, A .4, A .5, A .6, A .7, A .8, A .9, A .10, A .11, A .12, A .13, A .14, A .15, A .17\}$

$r_{111}=\{A .1, A .2, A .3, A .4, A .5, A .6, A .9, A .10, A .12, A .13, A .14, A .15, A .16, A .17\}$

$r_{120}=\{A .1, A .2, A .3, A .4, A .5, A .6, A .7, A .8, A .11, A .14, A .16, A .17\}$ 